MICROMACHINING OF POLYURETHANE (PU)

POLYMER USING A KrF EXCIMER LASER (248NM)

By

\title{
SARABPREET SINGH
}

Bachelor of Science in Mechanical Engineering

Sant Longowal Institute of Engineering and Technology

Sangrur, Punjab (India)

2007

\author{
Submitted to the Faculty of the \\ Graduate College of the \\ Oklahoma State University \\ in partial fulfillment of \\ the requirements for \\ the Degree of \\ MASTER OF SCIENCE \\ May, 2011
}




\section{MICROMACHINING OF POLYURETHANE (PU)}

POLYMER USING A KrF EXCIMER LASER (248NM)

Thesis Approved:

\begin{tabular}{c} 
Dr. Sandip Harimkar \\
\hline Thesis Advisor \\
Dr. Ranga Komanduri \\
\hline
\end{tabular}

Dr. Satish T.S. Bukkapatnam

Dr. Mark E. Payton

Dean of the Graduate College 


\section{SUMMARY}

Polyurethane (PU) polymer, a thermoplastic material, melts when exposed to laser light due to a variety of primary and secondary structures. Pattern and/or inducing a significant chemical change of the surface of polyurethane impacts a number of technologies, such as tissue engineering, MEMS devices, semiconductor manufacturing. At present, polyurethane (PU) is considered to be a best compromise material available, in terms of biocompatibility, mechanical flexibility and strength. Excimer laser micromachining is an appropriate tool for an alternative approach to conventional machining. It is highly desirable to relate the material removal rate with etch rate and ablation depth for understanding the ablation dynamics. In this investigation, micromachining of polyurethane polymer is conducted using a short pulse (FWHM $=25$ ns) $\mathrm{KrF}$ Excimer Laser (248 $\mathrm{nm}$ wavelength) that generates laser energy in the range of 100 to $650 \mathrm{~mJ}$. The machined surfaces were examined using conventional optical microscopy, surface profilometer and laser interference optical microscope (MicroXAM). The influence of the operating parameters, such as input energy, number of pulses, and environment on resulting micromachined geometries is also studied. Simple as well as complex geometries, such as micro-fluidic channels, micro-gears, part geometries used in medical applications, and electrical circuits were micromachined. 


\section{ACKNOWLEDGMENTS}

I would like to acknowledge and express my sincere appreciation to my advisor, Dr. Sandip Harimkar, for his everlasting guidance, intelligent supervision, inspirational attitude and mentorship for providing me with the opportunity to work, learn and perform research in the field of laser micromachining of modern materials. I would like to thank

all my committee members for being there when I needed them the most, especially Dr. Ranga Komanduri and also Dr. Satish Bukkapatnam. I wish to extend my gratitude and special thanks to my brother Amanpreet Singh for encouragement and moral support. Thanks are also due to Gary Thacker, Vamsi and Abhinay for their excellent work ethics and suggestions.

I wish to express my sincere thanks to my parents for everlasting family support, confidence, encouragement and positive attitude.

Finally, I would like to thank the Department of Mechanical and Aerospace Engineering (MAE) for accepting and providing me with the opportunity to pursue Master of Science at Oklahoma State University. 


\section{TABLE OF CONTENTS}

TITLE

PAGE NO.

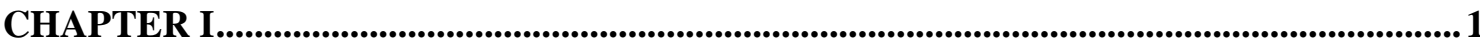

INTRODUCTION TO THE LASER .............................................................................................. 1

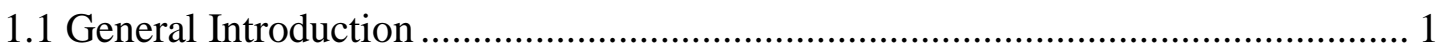

1.2 History of LASER

1.3 Working Principle

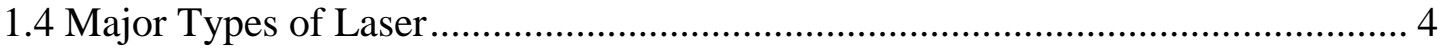

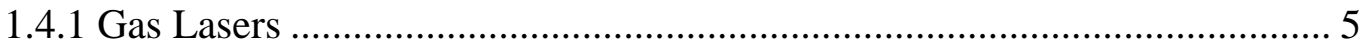

1.4.2 Chemical Lasers ................................................................................ 5

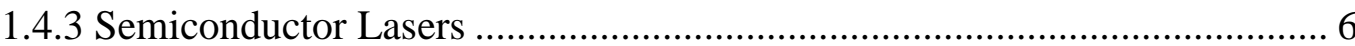

1.4.4 Metal-vapor Lasers ............................................................................ 7

1.4.5 Solid-State Lasers ................................................................................. 7

1.4.6 Other Laser Types .............................................................................. 8

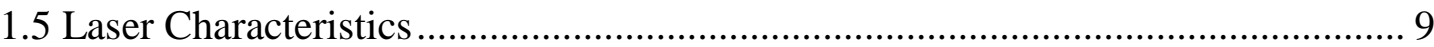

1.5.1 Wavelength Spectrum ........................................................................ 10

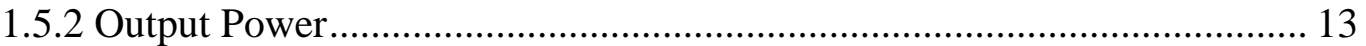

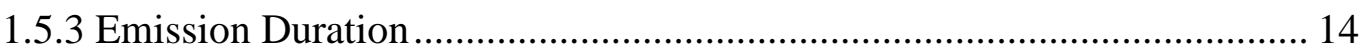

1.5.4 Beam Divergence and Size.................................................................... 15 


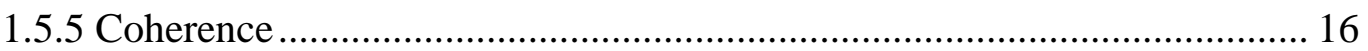

1.5.6 Power Requirement and Efficiency........................................................ 17

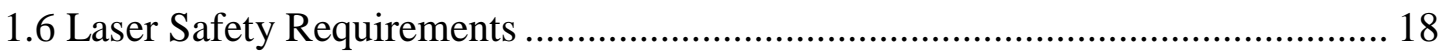

CHAPTER II ............................................................................................................................. 20

EXCIMER LASER MICROMACHINING ................................................................................20

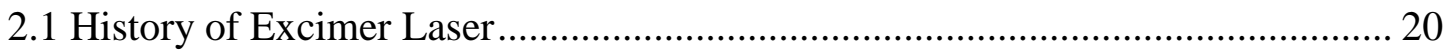

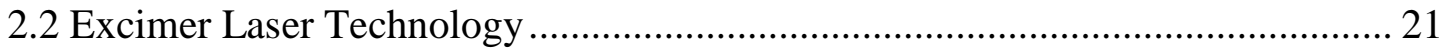

2.3 Excimer Laser Working Principle ………………………………………..... 24

2.4 Micromachining with Excimer Lasers ................................................................ 27

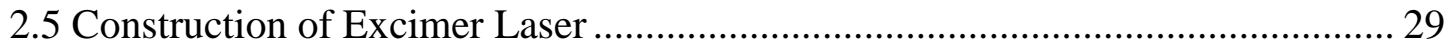

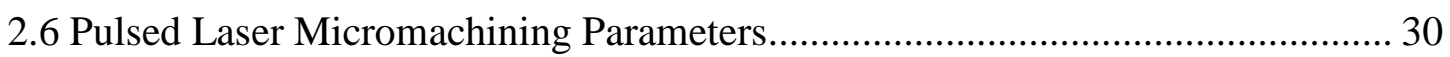

CHAPTER III...........................................................................................................................................31

BASICS OF LASER-MATERIAL INTERACTION..................................................................31

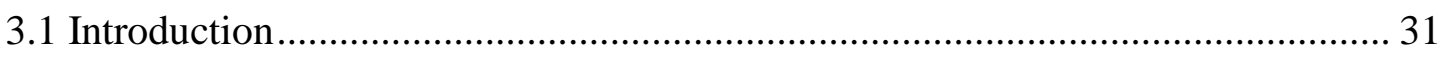

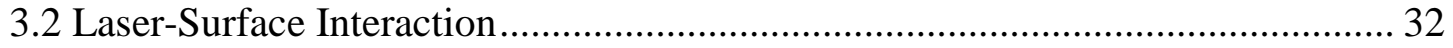

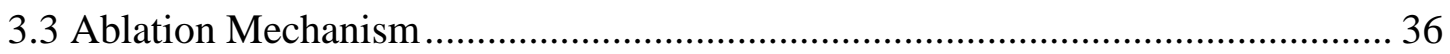

CHAPTER IV .......................................................................................................................

LITERATURE REVIEW ................................................................................................................39

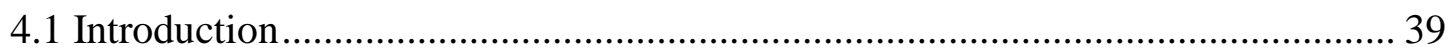

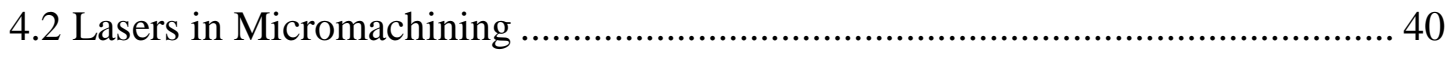


4.2.1 Underwater Laser Micromachining.................................................... 45

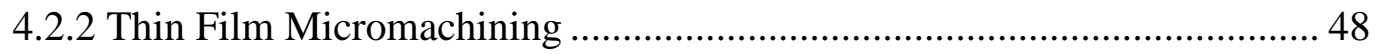

4.3 Excimer Laser 2D/3D Patterning ....................................................... 50

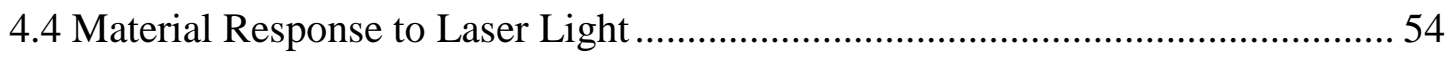

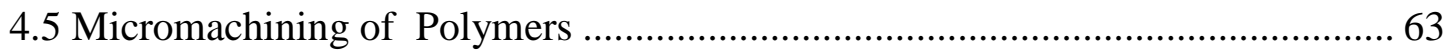

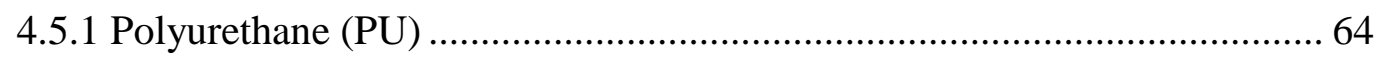

4.6 MEMS Devices and Patterns in Laser Micromachining................................... 67

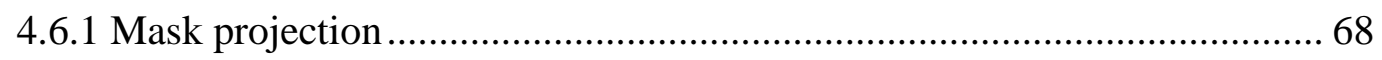

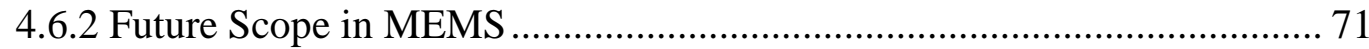

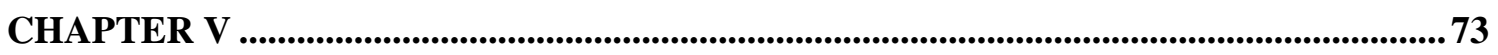

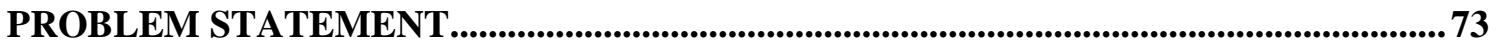

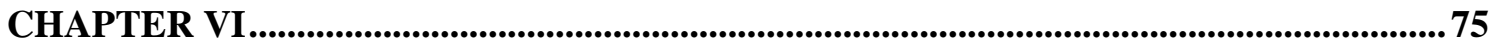

EXCIMER LASER EXPERIMENTAL SETUP AND OPERATING PROCEDURE........ 75

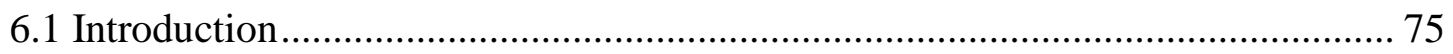

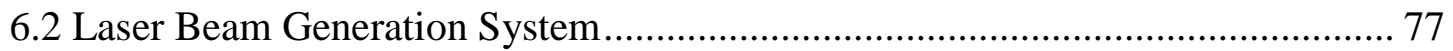

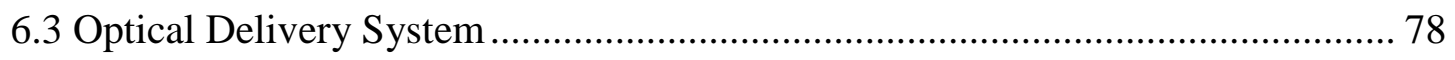

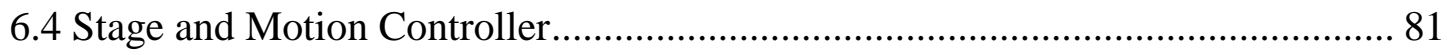

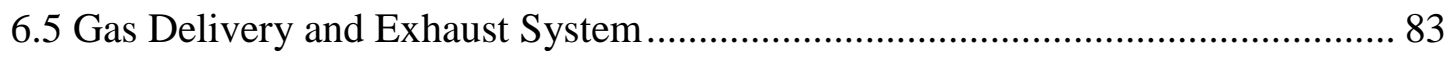

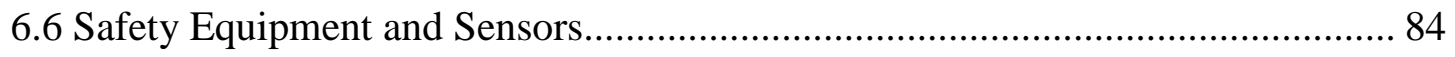


CHAPTER VII ..................................................................................................................................... 85

METHODOLOGY …............................................................................................................... 85

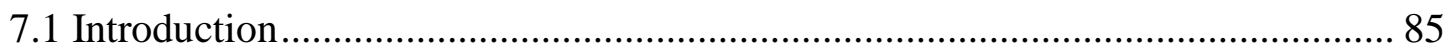

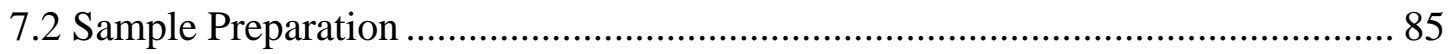

7.3 Determination of Laser Fluence, Average power and Focus Point ....................... 86

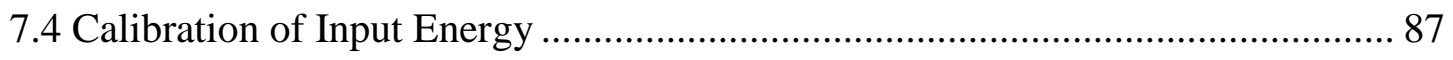

7.5 Laser Micromachining Under Different Environments ........................................ 87

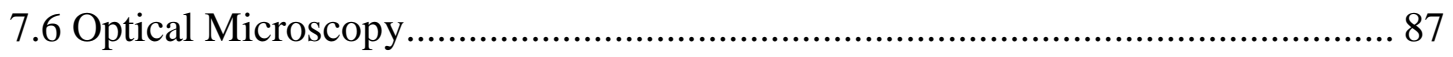

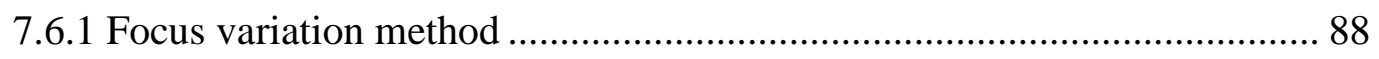

7.7 Surface Mapping using Surface Profilometer........................................................ 88

7.8 MicroXAM Laser Interference Microscopy ……………………............... 89

CHAPTER VIII.............................................................................................................................90

EXCIMER LASER MICROMACHINING OF POLYURETHANE ....................................90

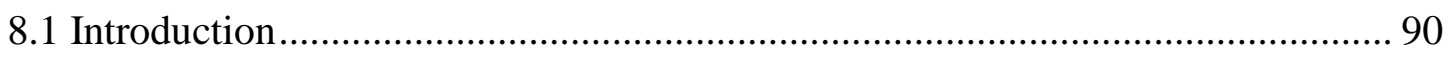

8.2 Ablation Characteristics of Polyurethane …………………………...................... 91

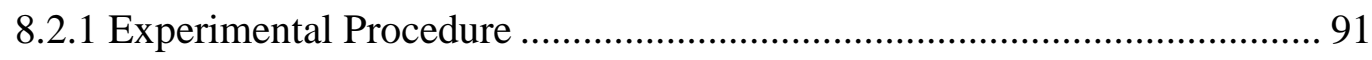

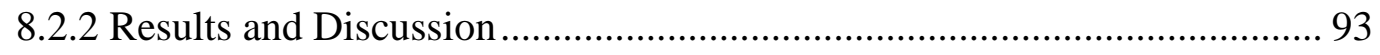

8.2.2.1 Change in Ablation Depth .......................................................... 96

8.2.2.2 Ablation Depth Comparison: Air Vs. Underwater ....................... 101

8.2.2.3 Depth profiles in air and underwater......................103

8.2.2.4 Change in Hole Diameter......................................................... 112 


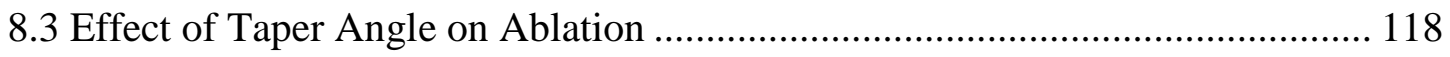

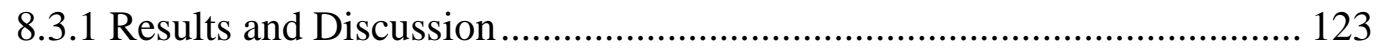

8.4 Two-dimensional (2D) Pattern Generation...................................................... 125

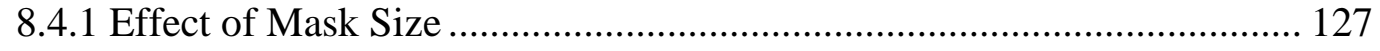

8.4.2 Ablation Mechanism in Etched Pattern....................................................... 131

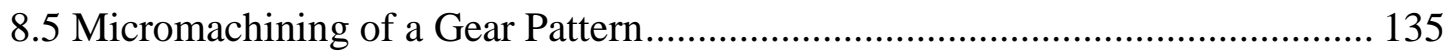

8.5.1 Scaling Effect of Mask Diameter ......................................................... 135

8.5.1.1 Results and Discussion .......................................................... 139

8.5.2 Effect of Mask Diameter on Seam Quality ……….................................... 141

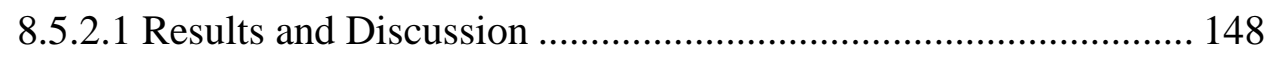

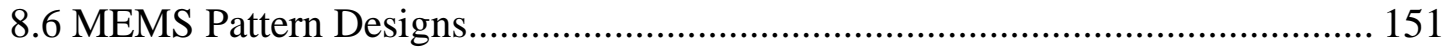

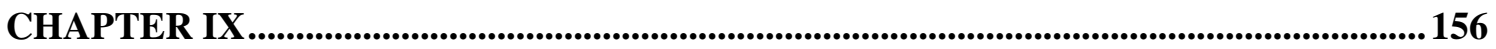

SUMMARY AND CONCLUSION...........................................................................................156

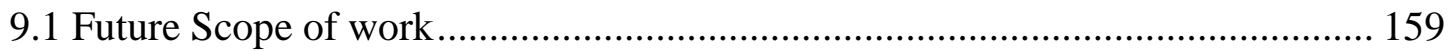

REFERENCES ............................................................................................................................160 


\section{LIST OF TABLES}

TITLE

Page No.

Table 1 Laser wavelengths for ultraviolet spectra ………………………………..... 11

Table 2 Laser wavelengths for visible spectra ……………………….................... 12

Table 3 Laser wavelengths for near-infrared spectra ................................................... 12

Table 4 Laser wavelengths for mid-infrared and far-infrared spectra ............................. 13

Table 5 Different types of excimer lasers and their wavelength .................................... 23

Table 6 Thermo-physical properties of glass, aluminum, and silicon ................................ 61

Table 7 Lambda Physik COMPex 205i Excimer laser specifications .............................. 78

Table 8 Properties of polyurethane (PU) polymer .......................................................... 93

Table 9 Experiments for a sample gear pattern ........................................................ 136 


\section{LIST OF FIGURES}

\section{TITLE}

Page No.

Figure $1 \mathrm{~A}$ schematic showing the of working principal in laser stimulation 3

Figure 2 Commercially available major types of lasers...................................................... 4

Figure 3 Important output characteristics of a laser...................................................... 9

Figure 4 Different spectrum regions illustrating laser wavelengths $\left(1 \mathrm{~nm}=10^{-9}\right.$ meter $) . .11$

Figure 5 Rayleigh range, beam diameter and beam divergence angle $(\theta) \ldots \ldots \ldots \ldots \ldots \ldots \ldots \ldots . . . . .15$

Figure 6 Coherent, spatially coherent and incoherent laser light................................... 16

Figure 7 Excimer laser short-lived principle illustration .............................................. 25

Figure 8 Comparison of Excimer laser micromachining with other commercially

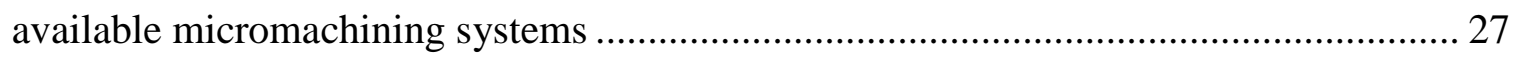

Figure 9 General laser machining principle.............................................................. 33

Figure 10 Illustration of laser beam interaction with a substrate ...................................... 34

Figure 11 An illustration of plasma vapor and debris formation in-air laser ablation...... 42

Figure 12 a) Plume development b) time development of the plume for a 2D simulation

Figure 13 Micromachining underwater environment illustration (Kruusing 1999) ......... 45

Figure 14 Laser etching of a NdFeB magnet in air and in water using Nd:YAG laser .... 47

Figure 15 Excimer laser interaction with a)thick polymer film and b)thin metal film..... 53 
Figure 16 Laser-matter interaction of a pulsed laser beam ........................................... 54

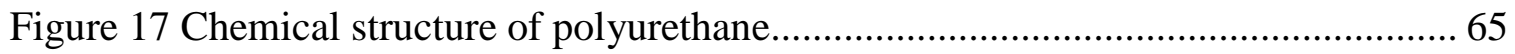

Figure 18 Important results about correlation between absorption coefficient and ablation

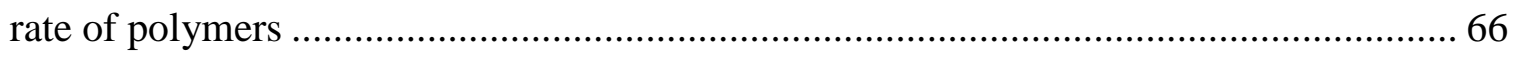

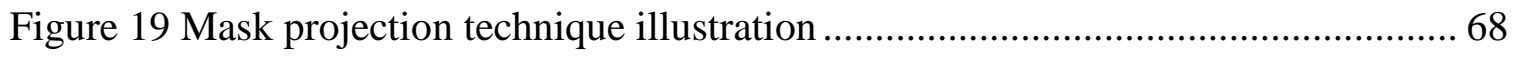

Figure 20 Glass probe array micro machined by laser ablation........................................ 70

Figure 21 SEM image of 3-D Electrodes on a circular SU-8 substrate ……………….... 70

Figure 22 Printed thin film micro-circuit pattern by excimer laser .................................. 71

Figure 23 SEM images of laser milling on glass realized with optimal parameters: (a) Rectangular cavity of $800 \mu \mathrm{m} \times 1200 \mu \mathrm{m} \times 400 \mu \mathrm{m}$, b) Close-up view of the surface finish ablated with the excimer laser and c) SEM image of a cavity milled in SiC ................... 71

Figure 24 a) Schematic of the laser system, b) Optical delivery system, c) Doublet and

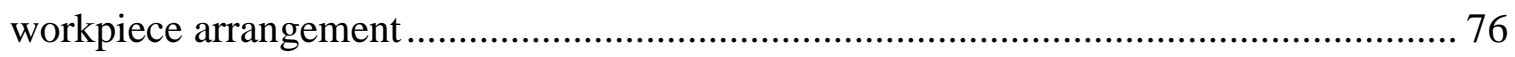

Figure 25 Schematic of experimental setup for excimer laser micromachining ……..... 77

Figure $26 \mathrm{~A} \mathrm{KrF} \mathrm{excimer} \mathrm{laser} \mathrm{generation} \mathrm{system} \mathrm{enclosure} \mathrm{..........................................} 77$

Figure 27 Schematic of the optical laser beam delivery system..................................... 79

Figure 28 Excimer laser optical beam delivery system ................................................. 80

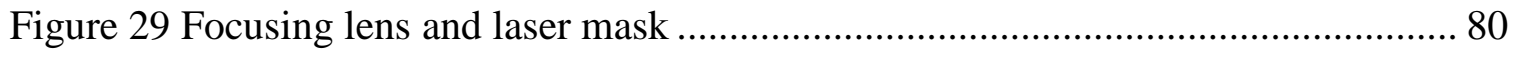

Figure 30 Computer workstation and translational $(\mathrm{X}, \mathrm{Y})$ stages .................................... 81

Figure 31 Translational stages and work sample .......................................................... 82

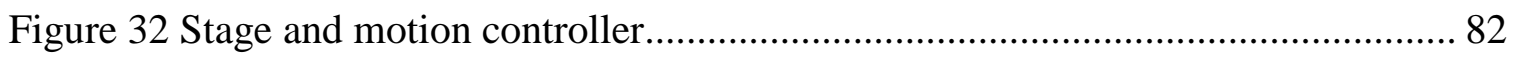


Figure 33 External control keypad and energy calibration device 83

Figure 34 Laser module gas exhaust system...................................................... 83

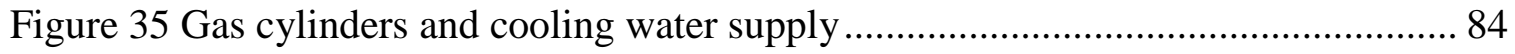

Figure 36 Safety sensor and hazard situation warning system ................................. 84

Figure 37 Schematic of the setup for micromachining underwater............................ 92

Figure 38 Optical micrographs of the top views of polyurethane samples ablated at pulse energy of $200 \mathrm{~mJ} / \mathrm{cm}^{2}$ at variable repetition rates $(1 \mathrm{~Hz}$ to $50 \mathrm{~Hz}$ or 5 to 250 pulses $) \ldots . .94$ Figure 39 Variation of ablation depth with increase in number of pulses at pulse energies of $200 \mathrm{~mJ}, 250 \mathrm{~mJ}$, and $275 \mathrm{~mJ}$. 95

Figure 40 For in air environment, at 25-to-100 Pulses (5 to $20 \mathrm{~Hz}$ ) optical micrographs (at 20x) showing ablation. 97

Figure 41 For air environment, at 175 to 250 Pulses (35 to $50 \mathrm{~Hz}$ ) optical micrographs (at 20x) showing ablation. 98

Figure 42 For underwater environment, at 25 to 100 Pulses (5 to $20 \mathrm{~Hz}$ ) optical micrographs (at 20x) showing ablation. 99 Figure 43 For underwater environment, at 175-to-250 Pulses $(35-50 \mathrm{~Hz})$ optical micrographs (at 20x) showing ablation. 100 Figure 44 Relationship between etch rate/pulse ( $\mu \mathrm{m} /$ pulse) and number of pulses $(\mathrm{N}) .108$ Figure 45 Laser micromachining in air shows variation in ablation depth Vs repetition rates 109 
Figure 46 Laser micromachining underwater showing variation in ablation depth with increase in number of pulses. 110

Figure 47 Variation of the diameter of the holes ablated with number of pulses at $150 \mathrm{~mJ}$, $200 \mathrm{~mJ}$, and $300 \mathrm{~mJ}$ for in air and underwater environments

Figure 48 Underwater ablation features on polyurethane sample ablated with 250 pulses @ 500mJ 114

Figure 49 For underwater micromachining, change in HAZ, TDZ and DRZ diameter of polyurethane sample 115

Figure $50 \mathrm{~A}$ illustration of the hole geometry cross section for calculating taper angle for hole walls 118 Figure 51 Variation of taper angle with repetition rates for $150 \mathrm{~mJ}$ pulse energy for in air and underwater. 120 Figure 52 Variation of taper angle with repetition rates for $200 \mathrm{~mJ}$ pulse energy for in air and underwater. 121

Figure 53 Variation of taper angle with repetition rates for $300 \mathrm{~mJ}$ pulse energy for in air and underwater. 121

Figure 54 Taper angle comparisons between in air and underwater ablation with increase in number of pulses 122

Figure 55 Effect of feed rate $(\mu \mathrm{m} /$ pulse $)$ on seam quality 126 Figure 56 Electric microcircuit pattern made using CimCAD software, ablated pattern at $E=250 \mathrm{~mJ}$, A-B) Optical microscope focus at surface of the seam, and at bottom of the seam 128 
Figure 57 3D view, Top view of a sample pattern and line profile of an edge. 129

Figure 58 A sample electronic microcircuit pattern

Figure 59 At 20x, Seam quality observed under "in air" environment for polyurethane substrate 132

Figure 60 At 20x, Seam quality submerged "under water" environment for polyurethane substrate 132

Figure 61 For in air ablation a) Focus at top surface of the seam b) Focus at bottom surface of the seam 133

Figure 62 A sample gear pattern of having an outer diameter of $2.4 \mathrm{~mm}$ 135

Figure 63 Different samples etched with scale variation and material for the design of the gear pattern after laser etching 136

Figure 64 For test 1; 3D surface profile for a gear etched with mask size of $1152 \mu \mathrm{m}$ on polyurethane sample (1 $\mathrm{mm}$ thickness) having $2.4 \mathrm{~mm}$ diameter

Figure 65 For test 2; 3D surface profile of a gear etched with mask size of $555 \mu \mathrm{m}$ on polyurethane sample ( $1 \mathrm{~mm}$ thickness) having $1.2 \mathrm{~mm}$ diameter 137

Figure 66 for test 3; 3D surface profile for a gear etched with mask size of $1152 \mu \mathrm{m}$ on PET sample (0.1 mm thickness) having $2.4 \mathrm{~mm}$ diameter 138 Figure 67 For test 4; 3D surface profile for a gear etched with mask size of $555 \mu \mathrm{m}$ on PET polymer sample ( $0.1 \mathrm{~mm}$ thickness) having $1.2 \mathrm{~mm}$ diameter 138 Figure 68 For test 1; 3D surface profile for a gear etched along with line profile at running seam for polyurethane sample (1 $\mathrm{mm}$ thickness) etched gear( $2.4 \mathrm{~mm}$ dia.) with mask size of $1152 \mu \mathrm{m}$ 
Figure 69 For test 2; 3D surface profile for a gear etched along with line profile at running seam for polyurethane sample (1 mm thickness) etched gear( $1.2 \mathrm{~mm}$ dia.) with mask size of $555 \mu \mathrm{m}$ 142

Figure 70 For test 3; 3D surface profile for a gear etched along with line profile at running seam for PET polymer sample $(0.1 \mathrm{~mm}$ thickness $)$ etched gear $(2.4 \mathrm{~mm}$ dia.) with mask size of $1152 \mu \mathrm{m}$ 143

Figure 71 For test 4; 3D surface profile for a gear etched along with line profile at running seam for PET polymer sample $(0.1 \mathrm{~mm}$ thickness $)$ etched gear $(1.2 \mathrm{~mm}$ dia.) with mask size of $555 \mu \mathrm{m}$ 144

Figure 72 For test no. 1, Seam/Edge quality of gear pattern a) Focus at top surface b) Focus at bottom surface 145

Figure 73 For test no. 2, Seam/Edge quality of gear pattern 146 Figure 74 For test no. 3, Seam/Edge quality of gear pattern a) At 20x focus on top surface b) At $50 \mathrm{x}$ 146

Figure 75 For test no. 4, Seam/Edge quality of gear pattern a) At 50x focus on top surface b) At 20x 147

Figure 76 For test no. 5, Seam/Edge quality of gear pattern a) At 100x focus on top surface b) At 50x 147

Figure 77 For test no. 1-6, at 20x Seam/Edge quality at the center of a) Test 1, b) Test 2, c) Test 3,d) Test 4, e) Test 5, f) Test 6 148

Figure $783 \mathrm{D}$ surface profile for a micro-switch contact with tolerance of $\pm 10 \mu \mathrm{m}$ material is 302 S25 stainless steel (Precision 2011) 151 
Figure $793 \mathrm{D}$ surface profile for a fine etched mesh having 30 holes $/ \mathrm{mm}^{2}$ sample thickness of $0.05 \mathrm{~mm}$ material is 304S15 stainless steel (Precision 2011). 152

Figure 80 3D surface profile for a microfluidic channels device showing specific depth etching with sample thickness of $0.38 \mathrm{~mm}$ using $302 \mathrm{~S} 25$ stainless steel ...................... 153

Figure $813 \mathrm{D}$ surface profile for a micro fuel cell using etched hexagonal holes with sample thickness of $0.25 \mathrm{~mm}$ and material is $316 \mathrm{~S} 11$ stainless steel. 154

Figure 82 3D surface profile for micro-contact of a battery with sample thickness $0.20 \mathrm{~mm}$ and material is $17-7$ stainless steel. 155 


\section{CHAPTER I}

\section{INTRODUCTION TO THE LASER}

\subsection{General Introduction}

In general terminology, LASER is an acronym for Light Amplification by Stimulated Emission of Radiation, it is an optical device that produces intense monochromatic beam of coherent light by emitting photons after being stimulated and amplified through an electromagnetic radiation process. The resulting wavelength of the optical beam range from X-rays to infrared and may come either as a pulsed, or as a continuous wave. The working principle of laser is based on stimulated emission of photons, a quantum mechanical phenomenon discovered (on theoretical grounds) by Albert Einstein in 1916 (Waerden 1967), in which a stream of photons passing through a medium absorbs energy with the capacity of electromagnetic radiation. The process of gaining energy by stimulated emission of photons is notable for its high degree of spatial and temporal coherence. 


\subsection{History of LASER}

In 1916, Albert Einstein (Waerden 1967), published a book on "Sources of Quantum Mechanics" explaining spontaneous and stimulated emission. He did not invent the device, but his work laid the fundamental basis for it. In 1958, based on Einstein's work, Charles H. Townes and his colleagues at Columbia university built a MASER: Microwave Amplification by Stimulated Emission of Radiation and published a detailed review of this device which greatly impacted the research of laser (Townes 2002). In 1960, Theodore H. Maiman built the first working laser at Hughes Research Laboratories in Malibu, California (Maiman 2000). Not long after, significant progress took place and lasers began to enter to commercial market (Winburn 1987). In the same year, Ali Javan invented the first continuous laser. This was followed by the first laser running in continuous operation at room temperature (no cooling necessary). In 1964, the first high power gas laser was developed by C.K.N. Patel of Bell Laboratories in Princeton, NJ. In 1971, the first Excimer laser was invented by Nikolai Basov, V. A. Danilychev and Yu. M. Popov, at the P.N. Lebedev Physical Institute in Moscow. They used a Xenon Dimer $\left(\mathrm{Xe}_{2}\right)$ as the gain medium, producing a $172 \mathrm{~nm}$ wavelength of radiation.

\subsection{Working Principle}

A typical arrangement of a conventional laser system consists primarily of a gain medium, an optical cavity, transmitter, output aperture, partial, and full reflectors. The primary goal of transformation of electrical energy into a highly dense energy beam was 
accomplished using stimulation and amplification of the beam constituents. Fig. 1 illustrates the phenomenon of laser stimulation below:

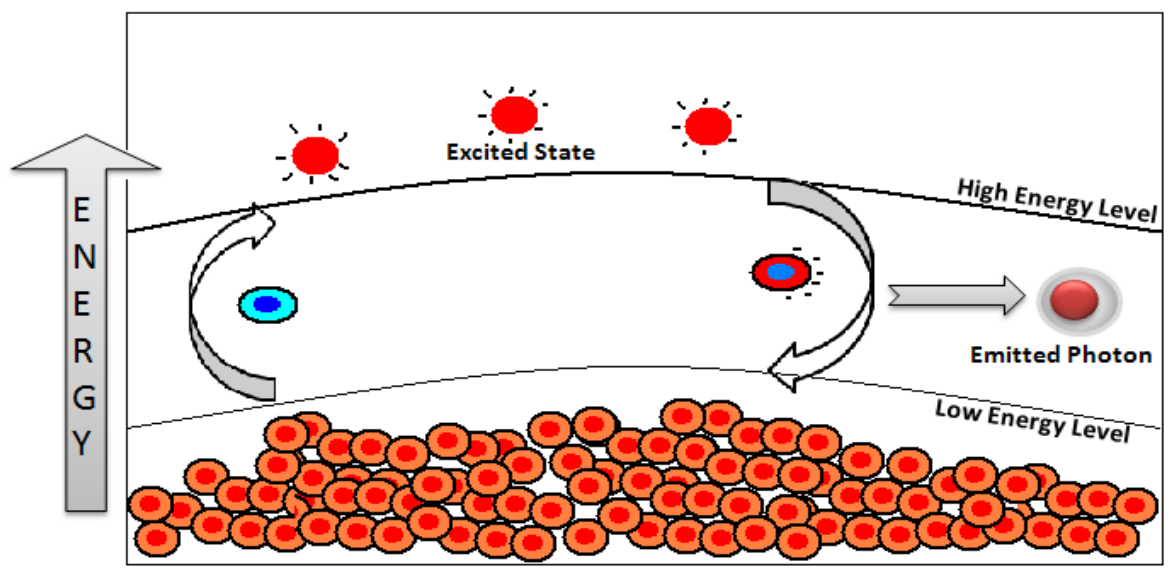

Figure 1 A schematic showing the of working principal in laser stimulation (Gould 1959)

The atoms and molecules generally exhibit low and high energy level states, in low energy state, the population of atoms observed is higher as compared to lightly populated high energy states. The atoms residing in their ground states at low energy levels can be excited and made to jump to high energy level state under the effect of external influences such as heating. Once excited, atoms reach their higher energy state, they can then go back to the ground state by releasing energy into photons; the presence of light emission called radiation of light. In laser, only those atoms can be stimulated to emit emissions which are in same phase with the incident radiation that has stimulated it in the first place. Therefore, by repeating the process, the increasing emissions multiply the population of highly excited atoms, thus exceeding the number of ground state atoms, a phenomenon known as population inversion. The resulting beam is a high powered, coherent light beam. 
Under normal laser stimulation, the proportion of excited atoms in this short-lived higher energy state depends on the level of excitement attained through initial intake of energy. Repetition of this concept yields a reverse for the ratio of number of atoms present in higher state to the number of atoms present in the lower energy state, in other terms population inversion.

\subsection{Major Types of Laser}

Various types of lasers can be classified according to many different criteria such as the type of lasing medium employed, operational wavelength, pumping source and the application of the laser. Commercially, the following types of lasers shown in Fig. 2 are available:

\section{MAJOR TYPES OF LASER}

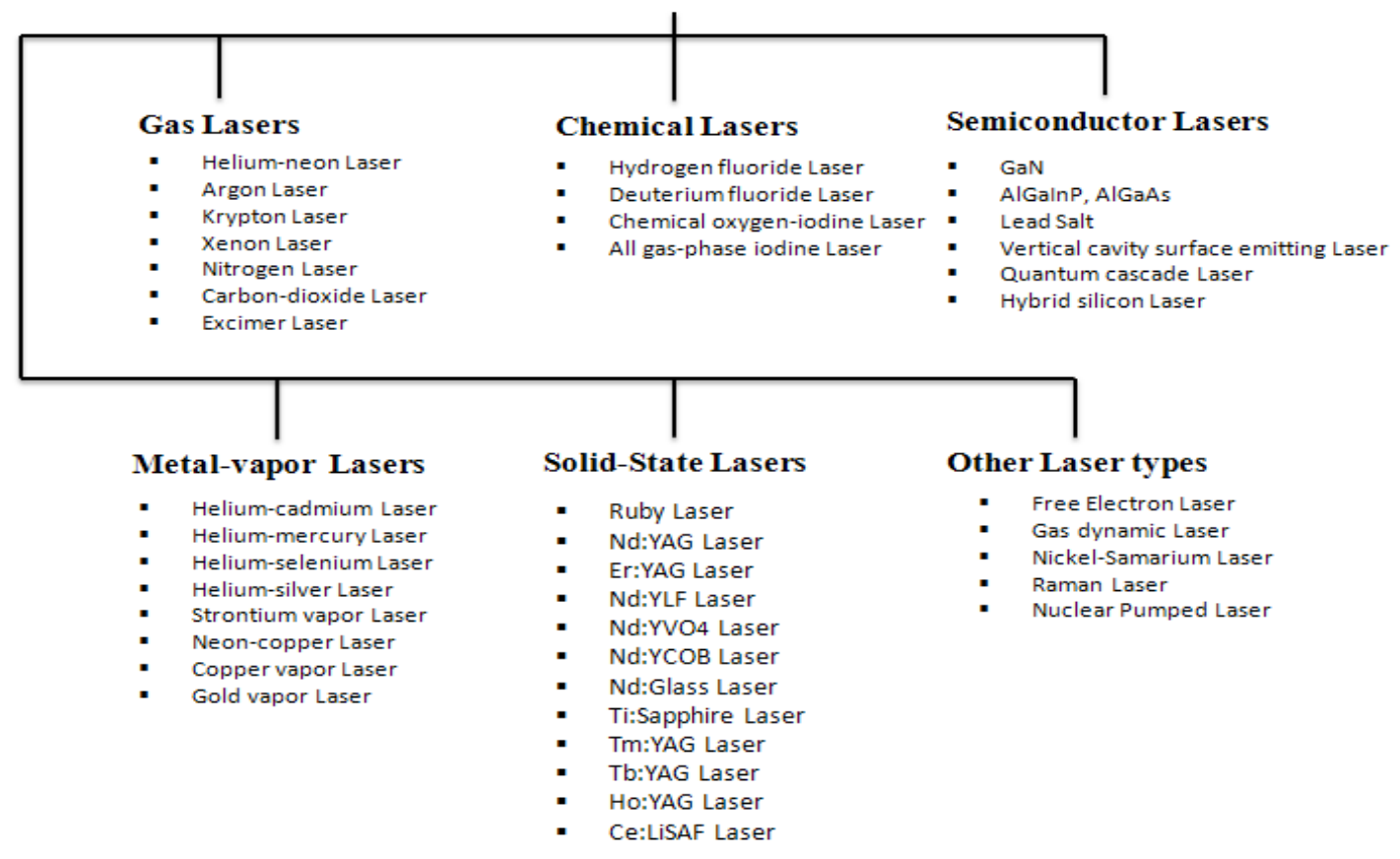

Figure 2 Commercially available major types of lasers (Weber 1999) 


\subsubsection{Gas Lasers}

Gas lasers produce light emission by discharging electric current through their gain medium which is usually filled with a gas. They can be further classified based on the mixture of the gas as neutral-atom, ion, or molecular type inside the gain medium. The area of application of a gas laser defines many of their operational aspects and control. The helium-neon $(\mathrm{HeNe})$ lasers are able to generate a wide range of wavelengths suitable for a wide variety of low cost applications with good beam characteristics. For rapid removal of material in industries, the carbon-dioxide lasers are used because of their ability to produce large energy inputs with efficiencies up to $9-10 \%$, which makes them suitable for metal welding and metal cutting operations (Endo 2006). Krypton and Xenon ion gas based lasers emit operational wavelength from $416 \mathrm{~nm}$ to $799.3 \mathrm{~nm}$ including UV and IR are considered well suited for scientific research. The nitrogen lasers emit wavelengths at $\sim 330 \mathrm{~nm}$ used primarily for many practical applications such as

optical pumping dye lasers and measurement of air pollution. Excimer lasers emit radiation at various wavelengths such as $193 \mathrm{~nm}(\mathrm{ArF}), 248 \mathrm{~nm}(\mathrm{KrF}), 308 \mathrm{~nm}(\mathrm{XeCl})$ and 353nm (XeF) mostly suited for ultraviolet lithography, manufacture of semiconductors, LASIK and eye surgery applications (Endo 2006).

\subsubsection{Chemical Lasers}

A chemical laser is a laser that outputs a continuous or pulsed wave of energy with the help of a chemical reaction. Chemical lasers are widely employed in industries for cutting and drilling applications due to high power reaching up to several megawatt 
levels. Common types of chemical lasers are the hydrogen fluoride laser, deuterium fluoride laser, chemical oxygen iodine laser (COIL), and all gas-phase iodine laser (AGIL). The hydrogen fluoride lasers produce operational wavelengths from 2.7 to 2.9 $\mu \mathrm{m}$ in which the chemical reaction consists of a burning jet of ethylene and nitrogen trifluoride $\left(\mathrm{NF}_{3}\right)$. Deuterium fluoride lasers produce operational transmittance of around $90 \%$ with wavelength outputs $\sim 3.6$ to $4.2 \mu \mathrm{m}$. The chemical oxygen-iodine lasers (COIL) operate at around $1.315 \mu \mathrm{m}$ with $70 \%$ atmospheric transmittance with the help of a chemical reaction in a jet of singlet delta oxygen and iodine (Gross 1976). The chemical oxygen-iodine lasers are employed mostly for scientific and material research applications. The all gas-phase iodine lasers (AGIL) operate at similar output levels as COIL at $1.315 \mu \mathrm{m}$ with the help of chemical reaction of chlorine atoms with gaseous hydrazoic acid (Onorato 1985). Due to explosive nature of the chemical reaction, the practical uses include weaponry and aerospace applications.

\subsubsection{Semiconductor Lasers}

The majority of semiconductor lasers are compact and based on a combination of $3^{\text {rd }}$ and $5^{\text {th }}$ group elements of the Periodic Table, namely, Al, Ga, ln, N, P, As, Sb. The emitted ranges of wavelengths produced are usually observed from $410 \mathrm{~nm}$ to $1600 \mathrm{~nm}$. Gallium arsenide (GaAs) is commonly used semiconductor material which produces operational wavelengths of $0.4 \mu \mathrm{m}$, Several similar semiconductor materials are also used such as GaAs, AlGaAs, lnGaAsP and lnGaAs (Weng 1999). The most common semiconductor used are $\mathrm{AlGalnP}$ and $\mathrm{AlGaAs}$ which produce operational wavelengths from $0.63 \mu \mathrm{m}$ to $0.9 \mu \mathrm{m}$ which makes them suitable for machining, medical and consumer 
electronics applications. An important application of semiconductor lasers is for pumping other high energy laser systems such as Nd:YAG lasers.

\subsubsection{Metal-vapor Lasers}

A metal-vapor lasers (MVL) are based on vaporization of a solid or liquid metal, such as cadmium, calcium, copper, lead, manganese, selenium, strontium and tin, vaporized with a buffer gas, such as helium. Among a wide variety of coherent sources currently employed, metal-vapor lasers, primarily self-terminating devices occupy a special place due to a unique combination of output parameters. The most common types of metal-vapor laser gain mediums consists of $\mathrm{HeCd}, \mathrm{HeHg}, \mathrm{HeSe}, \mathrm{HeAg}$, strontium, $\mathrm{NeCu}$, copper, and gold. For a helium-cadmium (HeCd) metal vapor laser the operational wavelength of $325 \mathrm{~nm}$ to $440 \mathrm{~nm}$ is produced, Similarly, $\mathrm{HeHg}$ at $567 \mathrm{~nm}$ to $615 \mathrm{~nm}$, HeSe at $225 \mathrm{~nm}$, strontium vapor laser at $430 \mathrm{~nm}, \mathrm{NeCu}$ at $248.6 \mathrm{~nm}$, Copper vapor laser at $510 \mathrm{~nm}$ to $578.2 \mathrm{~nm}$ and gold vapor laser produces operational wavelength of $627 \mathrm{~nm}$ are used (Christopher 1999). One of the most promising areas for MVLs is atmospheric optics: atmospheric gas spectroscopy and analysis, and remote sensing of the atmosphere with the aim of measuring its parameters.

\subsubsection{Solid-State Lasers}

Contrary to conventional gas medium or liquid medium lasers, the solid-state lasers use a solid gain medium such as neodymium-doped glasses and ruby crystals instead of a gas or a liquid. Solid state lasers are considered a separate class from semiconductor lasers which also employ a solid gain medium. The gain medium is 
formed by having an active crystalline material doped with a rare earth element, such as neodymium, erbium, and chromium. Due to the fact that excited ions of dopant material are not strongly coupled with thermal vibrations of crystalline lattice makes it viable for attaining laser ablation threshold at lower power inputs. In order to achieve good efficiency, pumping by an optical source is required to excite atoms by means of xenon flash-lamp or an arc lamp. The range of wavelengths varies between $660 \mathrm{~nm}-985 \mathrm{~nm}$ while using neodymium-doped YAG and titanium-doped sapphire (Keoechner 1999). Solid state laser applications include scientific research, defense weaponry, and material processing.

\subsubsection{Other Laser Types}

There are other laser types recently developed for advanced processing of materials, such as free electron laser, nickel-samarium laser, gas dynamic laser (GDL) and Raman laser. Free electron lasers operate using the concept of magnetic excitation of free electrons in which the electrons are excited by a large array of magnets where electrons are free to move within magnetic structure. This enables a wide variety of wavelengths to be generated which range from microwave, infrared, visible spectrum, ultraviolet to X-rays. Gas dynamic lasers (GDL) are based on relaxation of vibrational states where a particular energetic state exhibiting high energy require longer to relieve to a lower state than a less energetic state (Colin 2004). During the process of relaxation a hot gas is generated which expands through expansion nozzle and flows through a mirror where emission by stimulation takes place. Raman lasers are developed recently by utilizing amplification of photon scattering upon collision with a substance resulting in a 
secondary wavelength of the emitted light (Colin 2004). This light is a direct result by exchange of energy by photons upon collision which by a Raman laser can be amplified and reflected to produce a coherent laser beam. Raman laser and nuclear pumped lasers are a current topic of material processing and laser research in the scientific community.

\subsection{Laser Characteristics}

Fig. 3 highlights important characteristics of a common laser system:

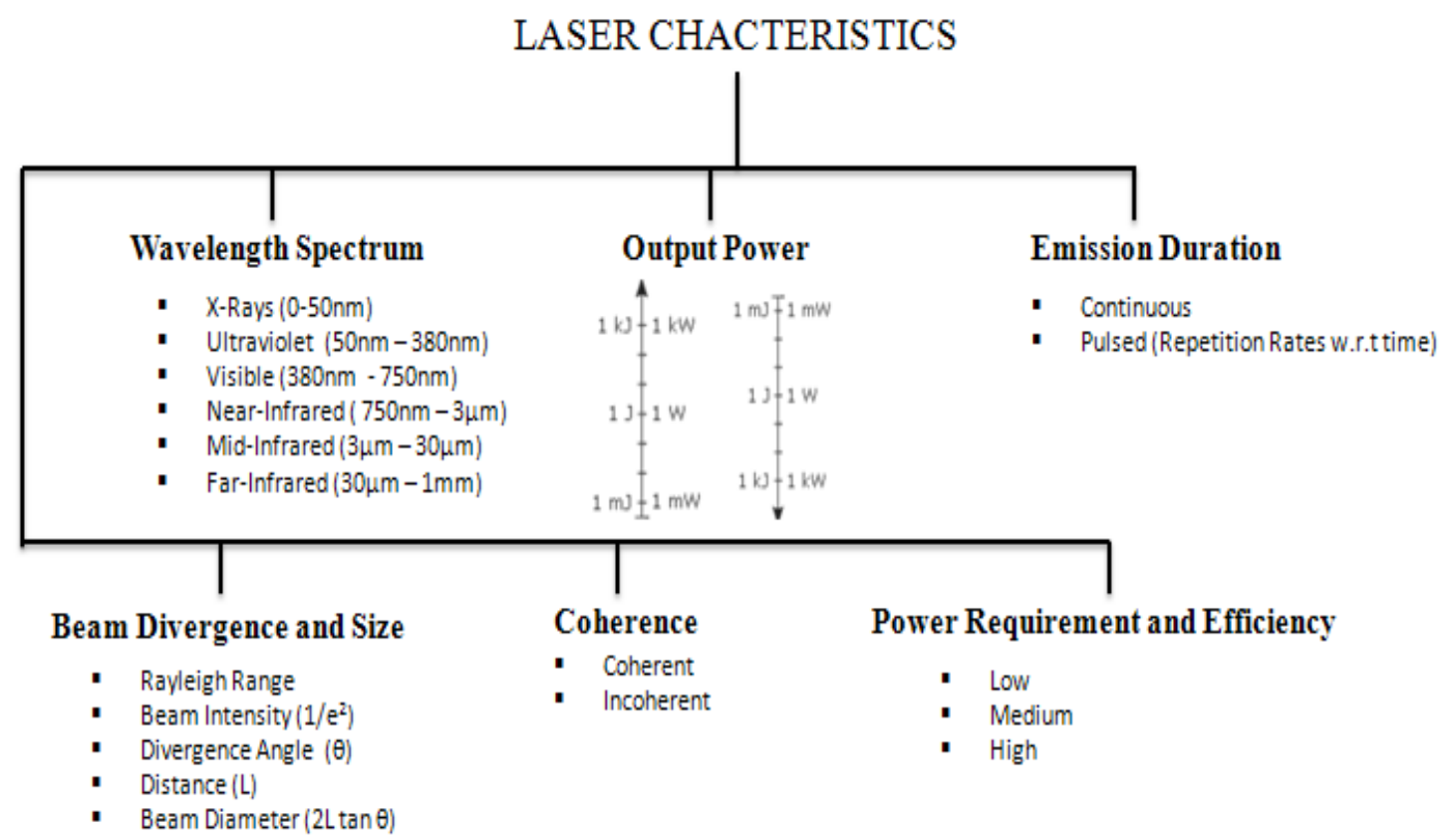

\section{Figure 3 Important output characteristics of a laser (Weber 1999)}

As mentioned earlier, there are a wide range of laser systems available commercially which differs from each other in terms of gain medium employed, amplification process used, and intended application area. There are some interrelated yet shared characteristics amongst the laser systems given in the following: 
- The active laser medium; which serves the purpose of amplification

- The energy pump; to achieve selective population inversion

- The optical resonator; consisting of reflective mirrors to resonate induced emission

The active medium or the laser medium is utilized to generate population inversion with the help of the energy pump to attain significantly large energy density inside the laser medium (Colin 2004). Thus the amplification effect is obtained and population inversion takes place. In general, the selection of a particular laser system is usually based on its output characteristics, such as wavelength, output power, duration of emission, coherence, beam intensity, and efficiency.

\subsubsection{Wavelength Spectrum}

Wavelength of a laser or other forms of radiation is a basic and a fundamental characteristic measure of an emitted light beam. Every kind of laser system exhibits a different wavelength depending upon several important factors such as type of gain medium used, optical interferences, laser optics, pumping source, amplification process and so forth. The wavelength of a particular laser system is measured in different spectrums ranging from X-rays to Infrared (IR) regions. Mostly, a laser light is generally referred to as a 'monochromatic' light, because it exhibits a single wavelength, however most lasers emit multiple wavelengths over a wide spectrum but due to narrow range of multiple minor wavelengths, a single wavelength can be considered dominant amongst narrow range of multiple minor wavelengths (Weber 1999). Figures 4 illustrates the 
various wavelength spectrums with values ranging from $50 \mathrm{~nm}$ (X-rays) to $1 \mathrm{~mm}$ (Infrared):
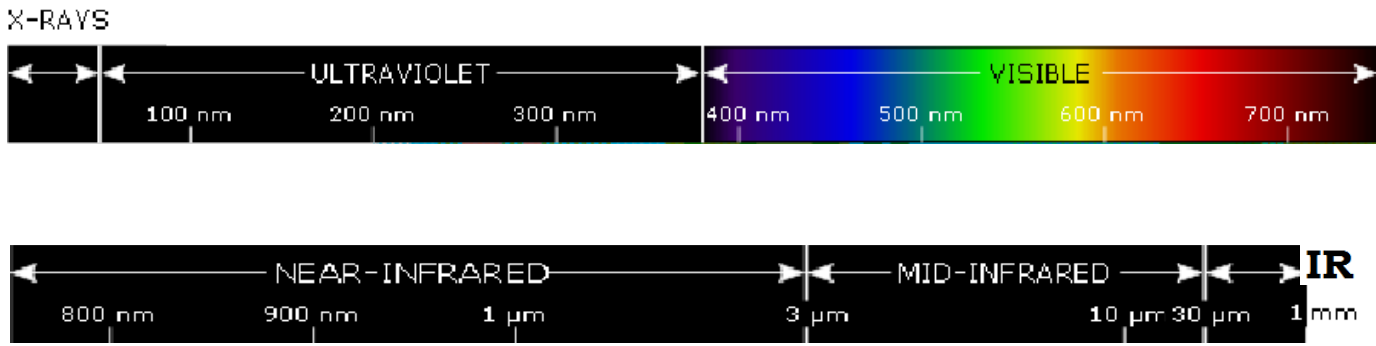

\section{Figure 4 Different spectrum regions illustrating laser wavelengths $\left(1 \mathrm{~nm}=10^{-9}\right.$ meter) (Weber 1999)}

Tables (1-4) show various laser media used for different spectrum regions providing a specific output wavelengths by various laser systems:-

Table 1 Laser wavelengths for ultraviolet spectra (Weber 1999)

\begin{tabular}{|c|c|}
\hline Laser System Type & Wavelength (nm) \\
\hline F2 Excimer & 157 \\
\hline ArF Excimer & 193 \\
\hline Nd:YAG (5th Harmonic) & 213 \\
\hline KrCl Excimer & 222 \\
\hline He:Ag+ & 224.3 \\
\hline KrF Excimer & 248 \\
\hline Nd:YAG (4th Harmonic) & 266 \\
\hline XeCL Excimer & 308 \\
\hline He-Cd & 325 \\
\hline Ne+ & 332.4 \\
\hline Nitrogen & 337.1 \\
\hline Ruby (doubled) & 347 \\
\hline XeF Excimer & 351 \\
\hline Nd:YAG (Tripled) & 355 \\
\hline &
\end{tabular}


Table 2 Laser wavelengths for visible spectra (Weber 1999)

\begin{tabular}{|c|c|}
\hline Laser System Type & Wavelength (nm) \\
\hline He-Cd & 441.6 \\
\hline Ar+ & 488 \\
\hline Copper-Vapor & 510.5 \\
\hline Ar+ & 514.5 \\
\hline Nd:YAG (doubled) & 532 \\
\hline Xe(3+) & 539.5 \\
\hline He-Ne & 543.5 \\
\hline Copper-Vapor & 578.2 \\
\hline He-Ne & 594.1 \\
\hline He-Ne & 611.9 \\
\hline Gold Vapor & 628 \\
\hline He-Ne & 632.8 \\
\hline Kr+ & 647.1 \\
\hline Ruby & 694.3 \\
\hline
\end{tabular}

Table 3 Laser wavelengths for near-infrared spectra (Weber 1999)

\begin{tabular}{|c|c|}
\hline Laser System Type & Wavelength $(\mathbf{n m})$ \\
\hline Nd:YAG & 946 \\
\hline Nd:YAG & 1064 \\
\hline He-Ne & 1152 \\
\hline Iodine & 1315 \\
\hline Nd:YAG & 1319 \\
\hline He-Ne & 1523 \\
\hline Er:Glass & 1540 \\
\hline Tm:YAG & 2010 \\
\hline Ho:YAG & 2900 \\
\hline Er:YSGG & 2790 \\
\hline Er:YAG & 2940 \\
\hline
\end{tabular}


Table 4 Laser wavelengths for mid-infrared and far-infrared spectra (Weber 1999)

\begin{tabular}{|c|c|}
\hline Laser System Type & Wavelength (nm) \\
\hline $\mathrm{He}-\mathrm{Ne}$ & 3391 \\
\hline $\mathrm{CO} 2$ & 10.6 \\
\hline $\mathrm{He}-\mathrm{Ne}$ & 3.391 \\
\hline $\mathrm{CO} 2$ & 10.6 \\
\hline Methanol & 37.9 \\
\hline Methanol & 118 \\
\hline Methylamine & 147.8 \\
\hline Methyl fluoride & 496 \\
\hline Methanol & 571 \\
\hline Methanol & 699 \\
\hline
\end{tabular}

\subsubsection{Output Power}

The most fundamental method of checking the performance of a laser system is to measure its power output or pulse energy output. For pulsed laser systems, the output power per pulse is an important characteristic in the determination of laser beam strength. For continuous laser systems the ratio of the flow of output energy with respect to time is taken under consideration. This refers to optical power output of a continuous wave of energy. A laser system's output energy is dependent upon several variables within the structure of laser equipment, mainly it depends upon the extent of laser energization and amplification, gain medium, pumping system, and laser optics (Colin 2004). The output of a laser can range from a few $\mathrm{mJ}$ to several mWs often measured in Watts (and often reported in terms of $\mathrm{nW}, \mathrm{mW}, \mathrm{W}, \mathrm{mJ} / \mathrm{cm}^{2}, \mathrm{~J} / \mathrm{cm}^{2}, \mathrm{~kJ} / \mathrm{cm}^{2}$, etc.) (Weber 1999). The energy 
(E) of the laser system is related to peak power $\left(\mathrm{P}_{\text {peak }}\right)$ and time (t) by the relation given in Equation (1.1) \& (1.2):

$$
\begin{aligned}
& \text { Energy }(\boldsymbol{E})=\boldsymbol{P}_{\text {peak }} \times \text { time } \\
& \text { Average Power }\left(\mathrm{P}_{\text {avg }}\right)=\mathrm{E} \times \mathrm{Hz}
\end{aligned}
$$

Where, $\mathrm{Hz}$ is the repetition rates with respect to time.

The laser systems under ultraviolet and visible spectra outputs energy values up to several Joules, followed by similar outputs by near-infrared spectrum laser systems. $\mathrm{CO}_{2}$ laser is well known for maximum output power in mid-infrared region at $\sim 1 \mathrm{~kJ}$.

\subsubsection{Emission Duration}

Most pulsed laser systems allow control of the repetition rates $(\mathrm{Hz})$ with respect to time which controls the emission duration. The duration of a pulse could vary from very short to long durations depending upon operational requirements. Generally, the values of pulse duration range from attoseconds $\left(10^{-18} \mathrm{~s}\right)$, picoseconds $\left(10^{-12} \mathrm{~s}\right)$, femtoseconds $\left(10^{-15} \mathrm{~s}\right)$ or milliseconds $\left(10^{-3} \mathrm{~s}\right)$ to several seconds or minutes. The desired emission duration can be achieved by modulating a continuous-wave light source. Different methods for the modulation of laser beam are used to achieve desired exposure of light emission, such as gain switching, mode-locking, high harmonic generation. Gain switching allows pulse generation by quickly modulating the laser gain via the pump power where higher the pump pulse energy, the shorter is the pulse build up time, and Thus the required pulse duration. Mode-locked lasers makes it possible to emit ultra-short pulses in which active/passive mode locking is employed where a laser resonator 
containing an active optical modulator rotates at high speed causes formation of an ultra short pulse. In high harmonic generation, the pump source contains a passive modelocked laser with a regenerative amplifier which when a very intense laser pulse is focused into a gas (at reduced pressure) can lead to strong nonlinear odd harmonics of the optical frequency of the pulse. This can be used for generating pulses with attoseconds $\left(10^{-18} \mathrm{~s}\right)$ durations in the extreme ultraviolet spectral regions. The advantages of femtosecond lasers include small to negligible HAZ, precise control of ablation threshold and geometry (Liang 2003).

\subsubsection{Beam Divergence and Size}

Beam divergence is an angular measure of the change in beam diameter of a circular beam when the distance from the optical aperture exit is increased, denoted by beam divergence angle $(\theta)$. The value of beam divergence angle $(\theta)$ increases if the beam is not focused properly due to some error in laser optics which cause increase in incident beam diameter on the specimen surface.

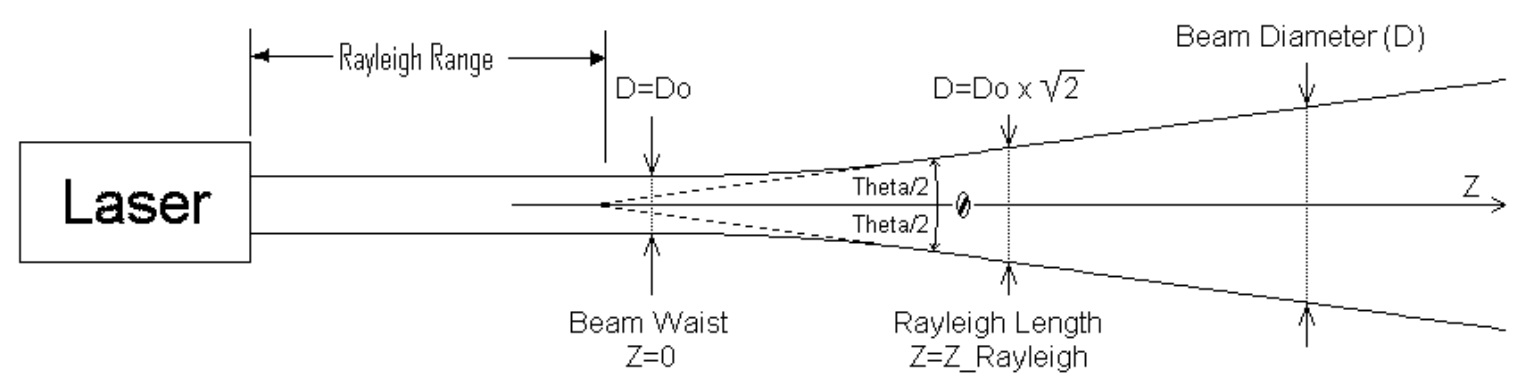

Figure 5 Rayleigh range, beam diameter and beam divergence angle ( $\theta)$ (Colin 2004) 
The beam divergence is a common problem among laser systems which is one of the reasons for poor beam quality because the divergence of a laser beam is proportional to its wavelength and inversely proportional to the diameter of the beam at its narrowest point. Thus, the beam divergence angle $(\theta)$ is expected to remain small to obtain best beam diameter and profile. All lasers are susceptible to beam divergence where the beam divergence angle $(\theta)$ is measured in milliradians $(\mathrm{mrad})$ or $\left({ }^{\circ}\right)$ degrees. The measurement of beam divergence angle can be made by measuring beam radius at different positions using a beam profiler. In some of the laser systems the divergence angle is controlled automatically by the smart laser optics.

\subsubsection{Coherence}
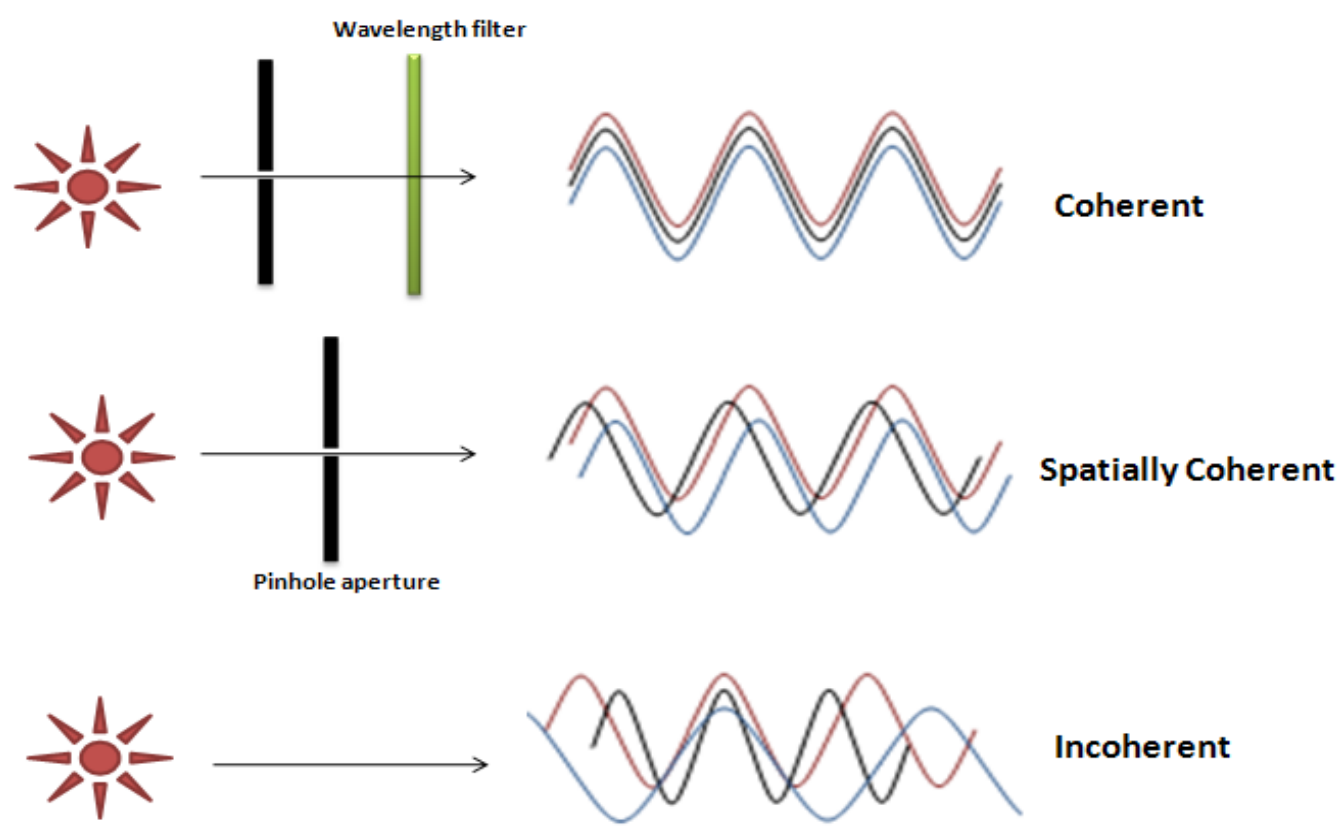

Figure 6 Coherent, spatially coherent and incoherent laser light 
In general, the coherence is an inherent property of waves which is a direct result of correlation between physical attributes and is largely affected due to constructive or destructive interference phenomenon. Two waves if added together in a single phase can form a larger wave having conforming peaks and valley curves. Similarly, two waves having different phases when added can form a relatively smaller wave due to inherent mismatching of physical attributes of the wave. In laser systems, laser light is a single phase coherent waves of photons, thus forms a monochromatic light. As the stimulated emission phenomenon means that the radiation is self-generating with possibility of low/high order beams enables several light interference effects.

\subsubsection{Power Requirement and Efficiency}

As most laser systems are employed in various industries and similar research fields, the efficiency of a laser system become an important criteria for laser system selection. Power requirement and efficiency are directly related to each other as efficiency is a measure of how well a laser system converts input energy into the useable laser light. As the mechanism of lasers involve exciting the atoms into an temporal excited state with higher potential energy by simultaneous stimulation, it becomes increasing difficult to attain higher efficiency due to this fundamental reason. For example in solid state lasers, a Nd:YAG (pumped by diode laser) usually have a $30 \%$ $40 \%$ efficiency by operating multimode with adequate thermal control of the diodes, and if the pump diodes themselves have about a $40 \%$ - $45 \%$ efficiency, thus it results in a net $18 \%$ of efficiency input directly from electrical energy to the diodes to output required laser beam output. Most industrial lasers can generate efficiencies from $0.1 \%$ up to $30 \%$. 
It plays an important role as an economical issue for a laser system's selection for a particular process.

\subsection{Laser Safety Requirements}

Although general purpose lasers are considered safe if operator follows the stated rules and regulations, but since low power lasers cannot efficiently ablate material, the higher power lasers are necessary to achieve desired material processing output. Therefore higher power lasers also invite a couple of safety concerns such as beam hazards, electrical hazards and toxic gas hazards. Generally, the lasers are classified into Class I, Class II, Class IIIa, Class IIIb and Class IV categories. Class I lasers outputs a nominal power at around $<0.5 \mathrm{~mW}$, Class II outputs $<1 \mathrm{~mW}$, Class IIIa $\&$ IIIb at $<0.5 \mathrm{~mW}$, Class IV lasers at $>0.5 \mathrm{~mW}$ of power output. The hazards related with each class of laser increase with increase in output energy can cause skin injury, skin burning, vision damage, skin inflammation and toxic gas inhalation. Moderate and high-power lasers are potentially hazardous because they can easily burn the retina of the human eye as the laser radiation cause potential injuries due to its thermal effects (Dale 2002). The fact that lasers operate in all different spectrums such as visible, ultraviolet and infrared can prove extremely dangerous to an unskilled operator working in the area. Direct laser radiation and indirect laser radiation reflected from highly reflective surfaces can also cause injuries to the operator. Precautions should be taken even when the laser is not operating because of the possibility of a gas leak or electrical hazard. Gas masks, laser safety goggles, hand gloves, and expert guidance should be employed along with common sense. As lasers operate under high electrical power, thus extreme care should be taken 
while working around naked electrical connections. Pilot tests should be conducted before the safe laser operation is determined. High voltage is the most lethal hazard associated with class IV lasers, due to which several fatal accidents have been reported all over the world. Thus, wherever possible, only authorized service professional should be allowed to open laser or perform maintenance procedures.

Following general safety guidelines(Dale 2002) are recommended for laser users:

- Abide by the two person buddy rule

- Do not wear metal objects during laser operation/maintenance

- Protective eyewear should be employed at all times

- Work with one hand at a time to prevent arm to arm electrical path

- Ensure that the operator's feet are not at ground potential

- Adhere to lockout/tag-out rule, close all gas cylinder valves

- Proper caution symbols should be used to alert operator

- Whenever possible, ensure that the electrical capacitors are fully discharged

- Keep a log of laser operation/maintenance to prevent future issues

- Cleanliness should be ensured around the laser equipment

Under normal conditions the laser operation and maintenance should be done by trained personnel. Periodically the laser system service should be done by authorized service professionals only. 


\section{CHAPTER II}

\section{EXCIMER LASER MICROMACHINING}

\subsection{History of Excimer Laser}

In the past few decades, the excimer laser has gained an important role in research and development efforts due to rapid demand in processing of a new generation of semiconductors, polymers and ceramics. Going back in history, the first excimer laser was invented and its operation realized in 1971 by Nikolai Basov, V. A. Danilychev and Yu. M. Popov, at the P.N. Lebedev Physical Institute in Moscow (Basting 2002). A xenon dimer (Xe2) excited by an electron beam was used to give light emission at 172 nm wavelength. Since then, several improvements were made using noble gas halides (originally XeBr), invented in 1975 by George Hart and Stuart Searles of the Naval Research Laboratory. A strong demand for miniaturization of electrical and electronic components fueled the research and development effort in excimer laser technology. Many types of excimer laser systems were developed over time with mantra of "smaller size and higher speed". Market demands for innovation drove the technological challenges to an extent far beyond the imagination in laser technology. 
Excimer laser ablation and direct etching of material by pulsed UV light has been an interesting subject of research activity since the early 1980s (Paterson 1999). Among many types of lasers developed during the development era, excimer laser was proved its way as a powerful and reliable piece of equipment for various application areas ranging from biomedical devices to semiconductors. Today the best application of excimer laser is seen in eye surgery and industrial micromachining of modern materials (Basting 2002).

\subsection{Excimer Laser Technology}

Excimer is a short term coined for Excited-Dimer, a name given to a diatomic molecule whose atom components are bound in excited energy state, and it refers to the gain-medium of the laser. Light beam produced by the excimer laser is emitted by a short-lived molecule, usually composed of one rare-gas and one halogen atom, called rare-gas halide. The laser emits a concentrated beam of light with the duration of $10 \mathrm{~ns}$ to several $100 \mathrm{~ns}$ in the ultraviolet (UV) region of the spectrum. The ability to operate excimer laser at different wavelengths such as $193 \mathrm{~nm}, 222 \mathrm{~nm}, 248 \mathrm{~nm}, 282 \mathrm{~nm}, 308$ $\mathrm{nm}, 337 \mathrm{~nm}, 351 \mathrm{~nm}, 427 \mathrm{~nm}$ and $713 \mathrm{~nm}$ along with selectable repetition rates makes it particularly suitable for a wide range of applications in semiconductors and polymers (Basting 2005). The wavelength of the laser depends on the transitions taking place inside the gain medium by the stimulated emission.

Excimer lasers have short wavelengths with high photon energies. This generally reduces the interaction time of the laser-material interaction which makes excimer lasers ideally suited for applications where smaller heat affected zone (HAZ) is highly desirable 
(Basting 2002). In $\mathrm{CO}_{2}$ and $\mathrm{Nd}: \mathrm{YAG}$ lasers, the photon energy is transformed directly from optical to thermal, i.e. the material is heated to melt and then vaporize. Whereas in excimer lasers, direct solid to vapor ablation is possible due to higher photon energies sufficient to break chemical bonds of the material being processed. This direct chemical dissociation of bonds eliminates the need for a liquid phase transition which allows small heat affected zones, thus better machinability. The main constituents of any excimer laser's gain-medium is a rare (noble) gas e.g. xenon or krypton, which is highly inert and doesn't form chemical compounds, normally; it is said to be in repulsive or disassociated, ground energy state (Colin 2004). However, if an electrical discharge is given, its energy climbs up to excited state, and forms a molecule with halides, e.g. fluorine or chlorine. This creates a temporal population inversion, that the total numbers of excited molecules has exceeded the normal disassociated atoms. The excited compound can release its excess energy by undergoing spontaneous or stimulated emission, resulting in a stronglyrepulsive ground state molecule which very quickly (on the order of a picoseconds) disassociates back into two unbound atoms. Most important types of excimer lasers operate using mixtures of $\mathrm{ArF}, \mathrm{KrF}, \mathrm{XeCl}$ and $\mathrm{XeF}$ (Basting 2005).

Excimer lasers are usually operated with a pulse rate of $1 \mathrm{~Hz}-50 \mathrm{~Hz}$ and with pulse durations of about 10-20 ns, although some operate as high as $200 \mathrm{~Hz}$ and $30 \mathrm{~ns}$ (Basting 2002). A very tightly-focused beam of UV radiation is emitted by an excimer laser. Their high-power ultraviolet output makes them useful for surgery (particularly eye surgery), lithography in semiconductor manufacturing, and dermatological treatment (Bores 2000; Basting 2002). It is generally considered as a cool laser type; as it does not 
heat up the surrounding air or surfaces. However, the sheer amount of UV light is already too much for most organic materials (such as the retina/cornea of the eye), resulting in the breakdown of the molecular bonds of the material. Excimer lasers come with quite large and bulky setups, which is a disadvantage in their medical applications, although their size is rapidly decreasing with ongoing developments. Most common types of excimer lasers are enlisted in Table 5:

\begin{tabular}{|c|c|}
\hline Excimer & Wavelength \\
\hline $\mathrm{Al}_{2}$ & $126 \mathrm{~mm}$ \\
\hline $\mathrm{Ki}_{2}$ & $146 \mathrm{~mm}$ \\
\hline $\mathrm{F}_{2}$ & $157 \mathrm{~mm}$ \\
\hline $\mathrm{Xe}_{2}$ & $175 \mathrm{~mm}$ \\
\hline $\mathrm{ArF}$ & $193 \mathrm{~mm}$ \\
\hline $\mathrm{CaF}_{2}$ & $193 \mathrm{~mm}$ \\
\hline $\mathrm{KrCl}$ & $222 \mathrm{~mm}$ \\
\hline $\mathrm{KrF}$ & $248 \mathrm{~mm}$ \\
\hline $\mathrm{Cl}$ & $259 \mathrm{~mm}$ \\
\hline $\mathrm{XeBr}$ & $282 \mathrm{~mm}$ \\
\hline $\mathrm{XeCl}$ & $309 \mathrm{~mm}$ \\
\hline $\mathrm{N} 2$ & $337 \mathrm{~mm}$ \\
\hline $\mathrm{XeF}$ & $351 \mathrm{~mm}$ \\
\hline
\end{tabular}

Table 5 Different types of excimer lasers and their wavelength (Basting 2005)

The excimer laser types mentioned in Table 5 release their excitation energy in UV spectrum (100-400 nm) in the form of radiated photons. The argon-fluoride (ArF), 
krypton-fluoride $(\mathrm{KrF})$ and xenon-chloride $(\mathrm{XeCl})$ are common types of excimer lasers that have very short-lived molecules. The fluorine $\left(\mathrm{F}_{2}\right)$ type is the only one which is not a rare gas-halide compound. Excimer lasers can also be categorized as chemical lasers, considering the reaction of the rare gas and a halogen mixture in their electrically excited state, triggered by an electron beam. For this reason, and also for their shorter wavelength than the already popular $\mathrm{CO}_{2}$ or $\mathrm{Nd}$ : YAG lasers, they draw interest for many applications mostly in eye surgery and semiconductor manufacturing.

\subsection{Excimer Laser Working Principle}

As mentioned before the short-lived diatomic molecule which forms from two atoms (at least one of which lies in its excited state) can give rise to laser light in the ultraviolet range. The two atoms are bound (forming a diatomic molecule) only when molecule is in a desired excited state. Often when a molecule drops to the ground state (a transition), which is the lower energy level, it falls apart and laser radiation is emitted. Fig. 7 shows the two energy level functions (curves) of a typical rare-gas halide as a function of the spacing between the two atoms: 


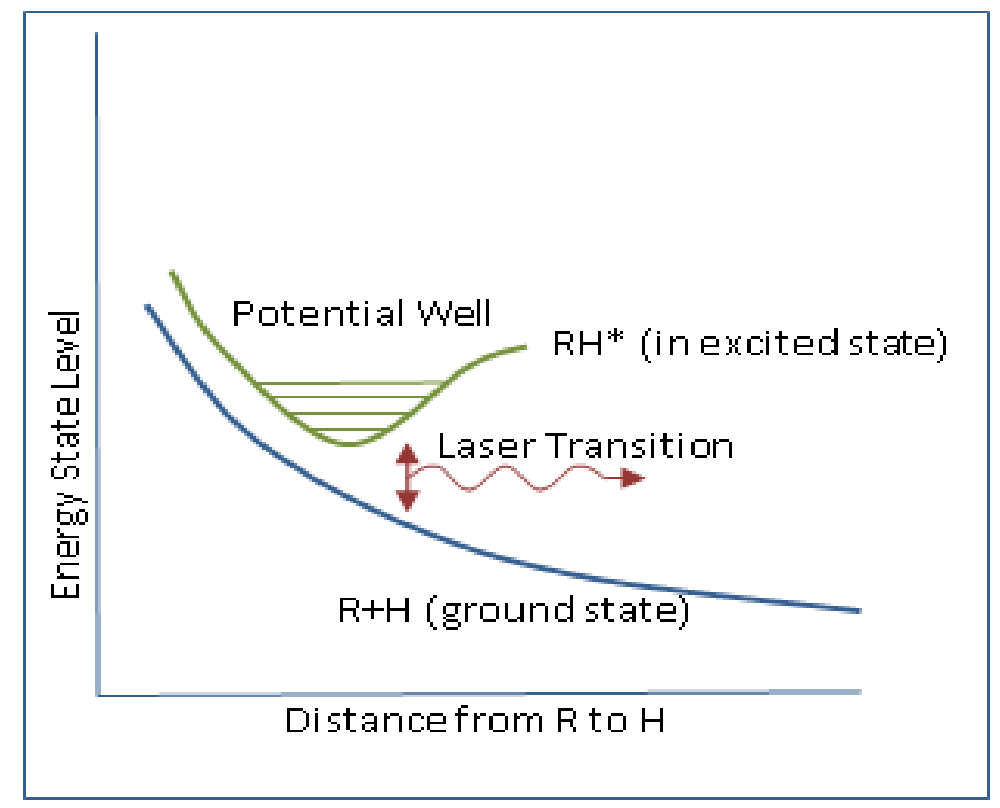

Figure 7 Excimer laser short-lived principle illustration (Winburn 1987)

The Fig.7 shows the two energy level functions (curves) of a typical rare-gas halide as a natural relation of the distance between the participating two atoms: $\mathrm{RH}^{*}$ is the notation for excited state of the collaborated atoms as a bounded molecule, while $\mathrm{R}+\mathrm{H}$ is the notation for the ground state of the same but unbounded molecule. $\mathrm{R}$ represents the rare-gas and $\mathrm{H}$ represents the halide (Winburn 1987). A dip in the excited state curve indicates meta-stable condition (a temporal but stable state until a sufficient disturbance comes to destabilize it), where the molecules are. Excited molecule has its energy at minimum when the two atoms are at a certain distance apart, trapped in a potential well (in it they can occupy several vibration levels as well). However, in the ground state, there is no bonding energy available to hold the two participating atoms together and the molecule would tend to fall apart. This state manifests rare gases in nature: they do not like to form compounds, even with highly reactive halogens. In 
general, excimer lasers are excited by applying electrical pulses through a gas mixture. About $90 \%$ of the mixture is a buffer rare gas usually helium or neon which does not take part in the ongoing reaction inside the laser. However, since pure halogens (e.g. $\mathrm{F}_{2}, \mathrm{Cl}_{2}$ or $\mathrm{Br}_{2}$ ) are highly reactive, they may come as a compound containing halogen, e.g. Nitrogen-trifluoride $\left(\mathrm{NF}_{3}\right)$. Electrical pulses release electrons and transfer energy to the laser beam, breaking up halogen molecules and causing formation of electronically excited molecules, e.g. excited xenon-fluoride $\mathrm{XeF}^{*}$. The $\left(^{*}\right)$ here indicates an excited molecule.

The reactions involved are complex depending on the type of gases used. They produce molecules which remain excited for short durations (a few nanoseconds) dropped to the ground state and dissociating, while releasing pulses of UV photon energy. These pulses are expected to last about tens or hundreds of nanoseconds, depending on the molecular kinetics as well as the duration of the driving electrical pulses. Excimer laser repetition-rate depends more on the supplied power (controlled by high-speed and high-voltage switches), rather than on the gas. Highest repetition rate is around $1 \mathrm{kHz}$, but more typical values are tens to few hundred Hz. Pulse energies range from about 10 micro joules $/ \mathrm{cm}^{2}$ to a few joules $/ \mathrm{cm}^{2}$. The average power, the product of the pulse energy times the repetition rate can reach about a couple of hundred watts or less. Note that in general the pulse energy tends to decrease with repetition rate. Fig.8 compares excimer laser with $\mathrm{Nd}$ :YAG and $\mathrm{CO}_{2}$ laser systems in term of important characteristics: 


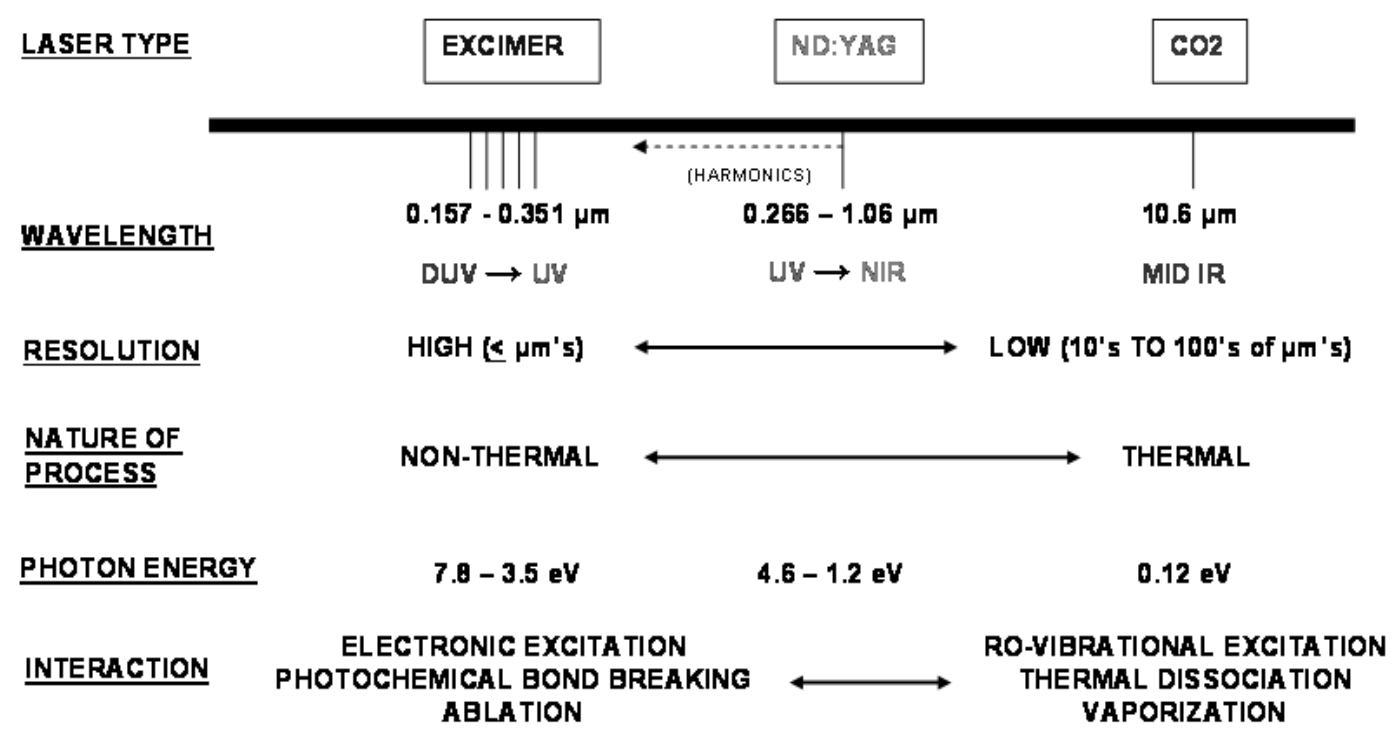

Figure 8 Comparison of Excimer laser micromachining with other commercially available micromachining systems (Weber 1999; Colin 2004; Basting 2005)

The energy from a laser is generally in the form of a beam of electromagnetic radiation (EM) which due to its inherent characteristics, such as wavelength, coherence, beam diameter, polarization and repetition rates can travel through optical delivery system of the laser.

\subsection{Micromachining with Excimer Lasers}

In general, laser machining is a material removal process that is achieved through energy-interactions between the laser beam and the target material. Laser machining has expanded beyond the conventional machining groups (Basting 2005) as follows:

1. Drilling (1-dimensional)

2. Cutting (2-dimensional) 
3. Shaping (3-dimensional)

4. General Laser Machining; Grooving and Marking Scribing

Conventional machining processes rely only on mechanical shearing stresses produced by a cutting element on the target material. Conversely, the laser machining is accomplished by transporting photon energy into the target material, in the form of thermal or photochemical energy to remove material (ablation) by melting and vaporization. This is one of the major differences between laser machining and conventional machining. When this machining is considered at a micrometer level it becomes micromachining. The continuing trend towards miniaturization and the use of advanced materials in medical devices have increased the need for new micromachining technologies. Laser is capable of playing a major role in micromachining industry because of the development of systems with smaller wavelengths and shorter pulses, this results in smaller spot sizes, and reduced heat input and HAZ. Machining with an excimer laser would improve the absence of thermal damage, enabling living tissues to heal without scarring. Because of this ability, excimer lasers found major applications in medical devices especially in eye surgery (Bores 2000). Apart from the applications in medical devices, excimer laser micromachining is widely used for applications in the manufacturing of MEMS devices, micro-gears, micro-pumps, micro-grids and micro structures in metals, polymers, glasses, and crystalline materials, down to a few $\mu \mathrm{m}$ in size. With advances in laser systems, and more efficient lasers coming up in the market, their applications are becoming increasingly more interesting and rapid. 


\subsection{Construction of Excimer Laser}

For general applications, the excimer laser usually consists of a fully reflective rear mirror and an uncoated output window that is partially reflective into the cavity and transmit the rest of the beam. The excimer laser gas chamber is usually sealed and highly resistive to any corrosive attack by halogen which operates until gas refill is mandatory. The volume of lasing medium is generally made larger in relation to the maximum volume discharge by laser per pulse. A recycling system, which regenerates the gas mixture often, helps extend the life of the gas fill. Maintaining proper gas mixture would also prolong laser energy shots. The total number of shots depends on the gas, and could reach some millions of shots for gas filled laser. A $50 \mathrm{~Hz}$ excimer laser would generate large amount of $(50 \times 60 \times 60=1,80,000)$ pulses per hour, until its gas is required to be replaced after a couple of hours usage. Excimer lasers are available as pulsed UV lasers with wall-plug efficiency of only a couple of percent. The commercial applications of excimer lasers have pushed manufactures to make more reliable lasers, although the use of hazardous (highly reactive) halogens still remains a concern. Laboratory excimer lasers are generally used with ultrahigh purity gases and are required to work for long durations with multiple wavelengths, in contrast to industrial excimer lasers which typically use process oriented specific gas mixtures. This calls for regular maintenance where operator has to discharge the old gas mixture, passivate the tube to remove contaminants, then pump out the mixture and replace it with a new laser gas mixture. This is generally a dangerous, complex and time consuming operation. 


\subsection{Pulsed Laser Micromachining Parameters}

When using a pulsed laser for micromachining tasks there are several important parameters to control in order to obtain desired results. Following are important parameters used in pulsed lasers:

- Laser repetition rate, $(\mathrm{Hz})$ : it is defined as the number of laser pulses per second

- $\quad \mathrm{X}-\mathrm{Y}-\mathrm{Z}$ machining velocity, $\mathrm{V}(\mu \mathrm{m} / \mathrm{sec})$ : it is defined as the displacement of work piece with respect to laser beam.

- Fluence, $\mathrm{F}\left(\mathrm{mJ} / \mathrm{cm}^{2}\right.$ or $\left.\mathrm{J} / \mathrm{cm}^{2}\right)$ : it is defined as the ratio of laser beam energy to the exposed area.

- Beam focalization angle, in degree $\left(^{\circ}\right)$ : it is defined as the measured angle from the center of a laser beam with a converging lens.

- Beam diameter, $\mathrm{D}$ in $(\mu \mathrm{m})$ : it is defined as the exposed diameter located after the focal point in the divergent part of the beam.

- Number of passes/cuts, $(\mathrm{N})$ : it is defined as the count of laser beam transitional passes over an area.

After micromachining, the other output characteristics are minimum ablation time, minimum angle of the walls, ablation (etch) rate, amount of recast or debris formation, HAZ area, and maximum ablation depth. 


\section{CHAPTER III}

\section{BASICS OF LASER-MATERIAL INTERACTION}

\subsection{Introduction}

In micromachining of materials, the pulse source of monochromatic UV light, such as excimer lasers can be used to ablate, vaporize, irradiate and modify surface characteristics. It is an irreversible process of chemical dissociation of atoms due to bond breakage caused by high energy photon collisions. In many applications the surface modification can result in exceptional surface properties such as high corrosion resistance, low surface roughness, improved biocompatibility, thin film deposition, coatings, heat treatment, elimination of surface defects and other topographical changes. However, due to the complexities involved in understanding the physical laser-material interactions, the lasers are rarely seen as a reliable source of heat (Perriere 2006). This complexity in laser-material interaction occurs due to combination of several chemical phenomenon occurring at the same time such as heating, absorption, melting, decomposition, evaporation, recoil effect, thermal shockwaves, piston effect and plasma shielding effect are a few to mention in current context. 
The laser-material interactions also involve photo-thermal and photochemical phenomena which affects ablation dynamics related to laser-material coupling. The physical process involved in laser material interaction forms the basis of understanding of the laser beam's interaction with matter and process limitations

\subsection{Laser-Surface Interaction}

In general, a laser can produce light radiation in pulsed or continuous mode with several important beam characteristics, such as high brightness, monochromaticity, narrow bandwidth, low divergence, high focusability with high spatial and temporal coherence. All of these important parameters affect the resulting phenomenon, namely, reflection, absorption, and transmission of light. Absorption and thermal heating starts as soon as incident laser beam strikes the surface which converts photon energy into thermal energy according to the key characteristics of the surface such as reflectivity, transmitivity, and absoptivity. The process however can be either photo-thermal, photochemical or a combination of both (Basting 2005; Perriere 2006; Sedky 2006). Lasers can irradiate light energy almost in all available spectra, i.e. X-ray, UV, Visible, IR, which allows flexibility to select a particular laser based on surface characteristics of a polymer, ceramic, or a metal. Fig. 9 is a schematic of a laser beam striking a substrate surface: 


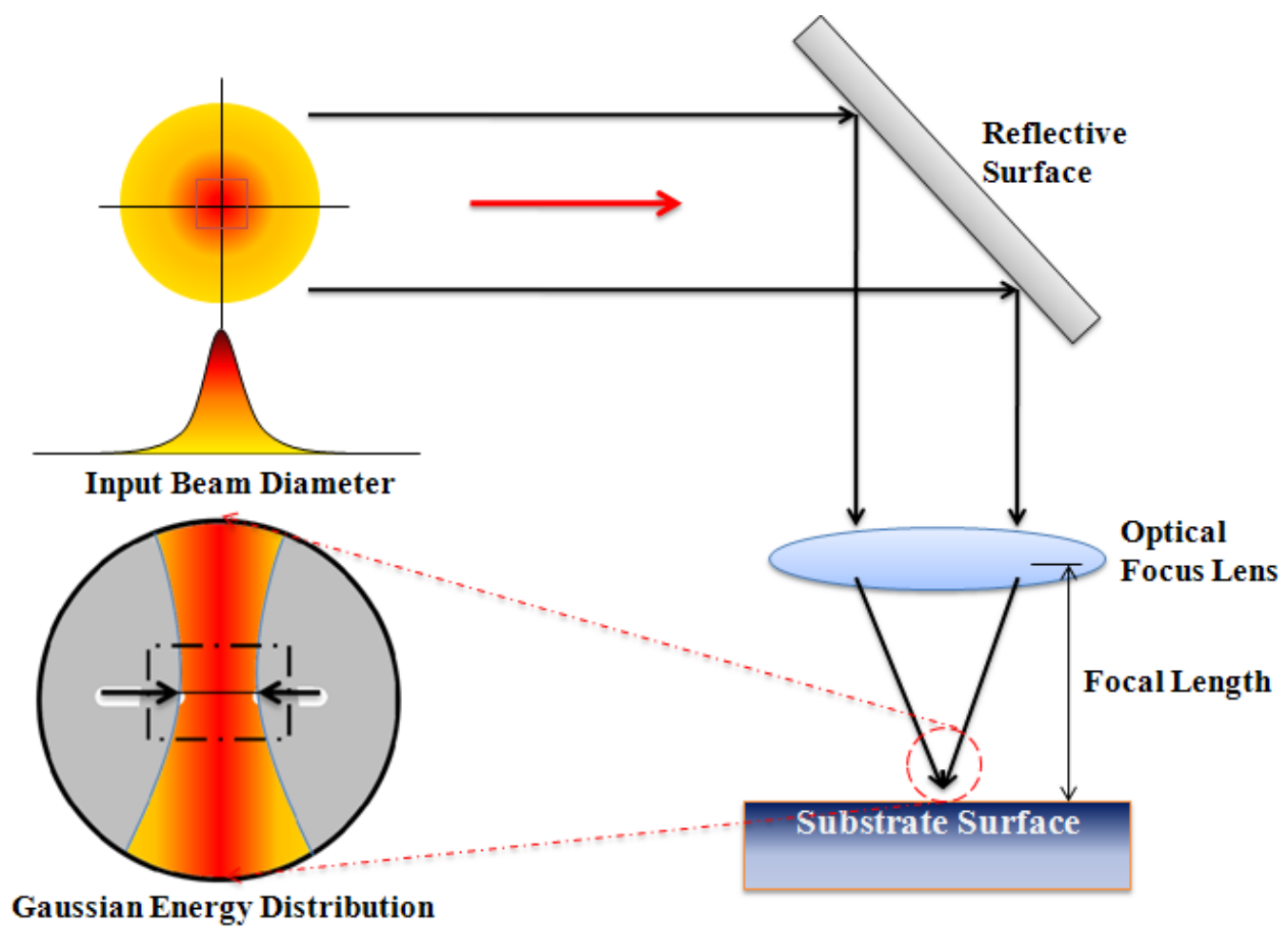

Figure 9 General laser machining principle

In a pulsed laser system, very high light energy can be delivered in a very short time period or pulse which allows non-equilibrium conditions to prevail during energy transfer between matter and photons. It is evident from the literature that various lasermaterial interaction phenomenon occurs at a specific set of parameters with respect to the material's physical, chemical, and environmental properties. In metals, high absorbability of ultraviolet laser radiation with extremely short pulse duration results in very fast cooling rates, as a result, smaller heat affected zones with refined and homogenous microstructure at the surface occur. Any material capable of interacting with laser light shows structural modifications due to electronic and lattice dynamics which plays an important role in understanding the laser-material interactions. The photons from the 
laser light beam transfer energy to available electrons on the surface of the material via inter- and intra-band electronic transitions. Heat transfer between surrounding electrons happen with electron-to-electron energy transfers which is largely based on the chemical properties and lattice structure of the material. In metals, highly reflective surfaces can cause electron scattering where the thermalization of electrons would result in interstructure electron-phonon interactions with each energy pulse. Similarly, in other materials the laser-material interactions depend on wavelength of the laser radiation and material properties, However, to induce any change on the surface the following conditions should be met by all means:

$$
\alpha+\beta+\tau=1
$$

Where, $\alpha$ is the reflectivity, $\beta$ is the absorptivity and $\tau$ is the transmitivity

Fig.10 illustrates the general laser beam interaction phenomenon with matter:

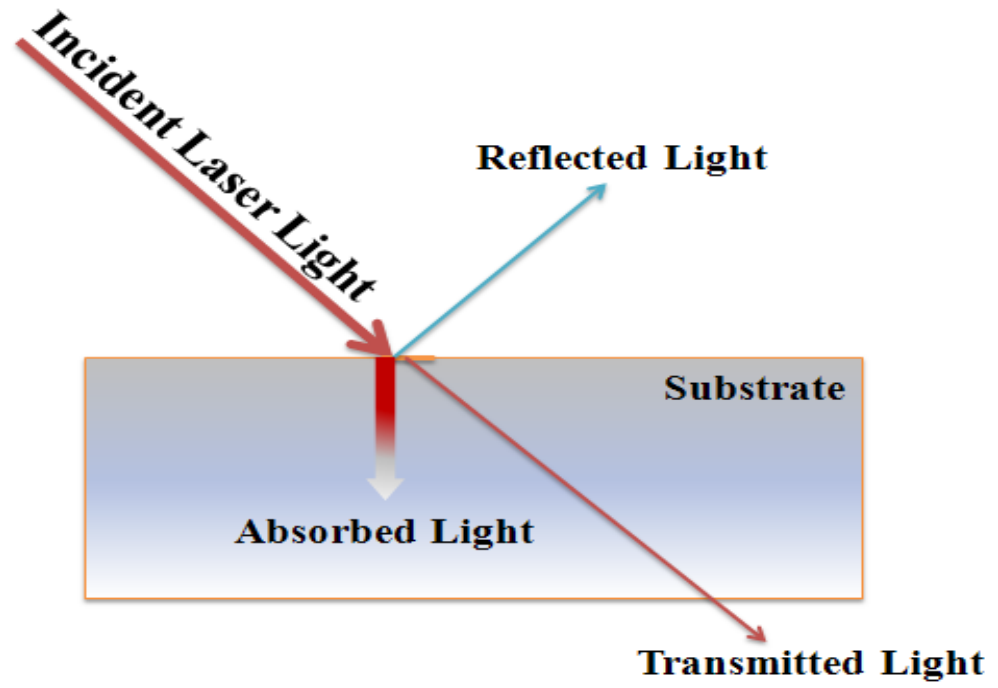

Figure 10 Illustration of laser beam interaction with a substrate 
As laser radiation is a form of electromagnetic radiation in which the beam strikes the surface where it may undergo reflectance $(\alpha)$, transmission $(\tau)$, or absorption $(\beta)$ process. The Beer Lambert's law plays an important role as it relates the absorption of light to the properties of the substrate material (Angelis 1999). The absorption coefficient $(\mu)$ depends on medium and wavelength of the incident radiation with intensity (I), which is given as follows:

$$
I=I_{o} e^{-\mu \cdot z}
$$

Where, $\mu$ is the absorption coefficient, I is the intensity

Although any significant visible change on the substrate's surface depend whether the deposited energy is enough to overcome ablation threshold of the material. In metals, the optical properties are determined by the availability of free electrons which are accelerated in the electrical field while gaining energy in which periodic changes cause them to re-radiate energy. Thus it causes high reflectivity of metals, unlike polymers where in the free electrons are lesser in number thus more absorption takes place due to weak interactions of inner electrons with the electromagnetic (EM) wave (Yang 2002; Perriere 2006).

In laser-material interaction, due to a high intensity of EM wave it provides high supply of energy from a laser source which can produce melting and evaporation effect in loosely bonded particles. The structural particles under the influence of EM wave can gain motion by the given electrical force and the absorption effect is governed by "the Inverse Bremsstrahlung Effect", which describes if there is enough energy travelling 
through a small volume of substrate. It can cause ionization and vaporization effect with plasma shield generation over the irradiated surface. The effect also incorporates a thermal shock wave which can travel though the work material and cause cracking or increased heat affected zone. In other words, it is caused by the emission of photons from the excited electrons. Therefore, when the strength of the laser field is high, it can be used to directly ionize the electrons of the substrate material through multi-photon absorption (Perriere 2006).

\subsection{Ablation Mechanism}

When a pulsed high power laser beam is made to strike over a substrate surface, the substrate undergoes ablation. Laser ablation is generally referred to as a process in which the laser beam cause enough transfer of energy from laser source to the substrate material surface to cause ionization and vaporization. The laser beam characteristics such as time, spatial distribution, frequency, and amplitude are most important factors in generating required ablation depth, phase transformations and other chemical changes. With short pulse durations the heat affected zone of the ablated area can be greatly reduced. Temporal modulation of laser beam energy can prove beneficial to improve machining quality. Similarly, the control of spatial distribution can allow elongated laser beam to travel distance without loss of energy or causing beam divergence. Frequency and wavelength differs from the rapid pulse repetition rate as different materials act differently with variation in frequency. Finally, the control of amplitude of the laser beam allows high power beams with dense energy field to travel to substrate surface which may also differ according to the absorption characteristics of the substrate material. In case of 
high power laser beams (up to several $\mathrm{J} / \mathrm{cm}^{2}$ fluences) with short nanosecond pulses cause extremely rapid melting and vaporization.

Laser ablation in ultraviolet regions is mostly governed by either or both of two major ablation phenomena, namely, photo-thermal and photo-chemical ablation. The type of ablation phenomenon is dependent upon the material's optical and mechanical properties, which greatly affect the etch rate (Desbiens 2007). Ablation phenomenon in metals and polymers involve various coupled and complex phenomenon, such as absorption, optical coupling of energy with matter, ejection of matter, fragmentation, shock waves, vaporization, plasma shielding, and the heat affected zones. In general, the surface of the substrate is heated past its melting and vaporization temperatures through linear single photon, multi-photon absorption, dielectric breakdowns and other complex mechanisms. In polymers or other soft materials, the vaporization temperature can be exceeded with only few extra pulses of energy, However, if the pulse energy is low, it can take longer duration to heat past the melting and vaporization temperatures. The laser ablation where the surface is irradiated till melting is called thermal ablation, and the process where direct vaporization occurs is referred to as non-thermal ablation. In nonthermal ablation, the possibility of plasma shield formation is significantly higher than that of thermal ablation. When the laser irradiance exceed past a threshold limit, the vapor of the ejected matter can become highly ionized to form plasma shield around the ablated area. This plasma shield basically restricts some proportion of the incident laser energy and radiation and increases in volume accordingly. At lower irradiance, the plasma formation exhibit transparency whereas at significantly higher irradiance the 
vapor cloud can become supersaturated and cause significant obstruction to ablation phenomenon. The material transport phenomenon, such as convection, evaporation, hydrodynamic motions are generally observed due to local temperature and pressure gradients. The variations in the temperature of the molten matter due to irradiance can lead to differential surface tensions and thermo-capillary effects. This is generally observed due to inhomogeneous laser intensity profiles.

In high power micromachining, mainly two modes of ablation mechanism are observed: 1) Matter ejection due to vaporization induced recoil pressure, 2) Meltevaporation due to high energy or short pulse durations (Colin 2004; Perriere 2006). The mechanisms of ablation studied in the past have shown that there are several distinct phenomenon involved in ablation of the surface due to laser-matter interaction such as energy absorption, photonic reflection, thermodynamic processes (melting, phase transformations), plasma shielding, shock waves, laser-plasma interaction, plasma heating, radiation and hydrodynamic expansions. At lower power, melting is most common with small amount vaporizing possible around the ablated zone. However, at higher power beam-material interaction, the thermal and pressure gradients cause rapid vaporization and plasma formation (Dehghanpour 2009). 


\section{CHAPTER IV}

\section{LITERATURE REVIEW}

\subsection{Introduction}

When a high power laser beam strikes a surface, the target surface melts and partially vaporize. This transition of photon energy from energetic laser beam to the surface of the substrate is a topic of widespread interest among researchers in various engineering disciplines. A comprehensive research effort is underway for understanding laser induced surface modifications and the nature of physical and chemical processes involved in it. Most experimental research efforts focus merely on analytical models based on many diverse assumptions ranging from non-equilibrium phase transitions to thermo-fluctuation sublimation of the particles from the surface due to pressure gradients generated by the laser light beam (Zhigilei 1998). For a substrate surface to be affected by laser requires the right kind of structural configuration, optical properties, and material properties. For example, in laser micro-drilling, the feasibility of a laser to be able to drill on a surface depends upon its wavelength, energy density, pulse shape of the laser beam (Zheng 2001). 
The laser beam which passes through the homogenizing optics, a mask and demagnifying optics breaks the chemical bonds and ejects the ablated products at supersonic velocities (Zheng 2001).

Techniques of high power laser cutting differ from low power laser cutting and etching processes, wherein the total number of photons entering the surface during each laser pulse are determined by laser power and pulse width (Zhigilei 1998). In surgical applications of laser ablation, the process can be used for controlled removal of tissue with highly precise control over thermal and mechanical damage to the surrounding tissues (Zhigilei 1998; Bores 2000).

\subsection{Lasers in Micromachining}

Among laser micromachining processes, ultraviolet (UV) laser ablation is a powerful and flexible technique which can be used for rapid patterning of small scale geometrical features that can be used on a number of different materials, such as polymers, ceramics, and metals (Callewaert 2003). Excimer lasers are generally short pulsed lasers that use a mixture of gases to generate output radiation and emission at a particular wavelength in the UV region of the spectrum (Tseng 2004). Femtosecond lasers produce even smaller pulse durations. In femtosecond lasers, the thermal effects are negligible because of the short duration of the pulse which allows enough time to cool the matter (Desbiens 2007). Liang et. al (2003) explored the fundamentals of femtosecond lasers to observe the integration of interference phenomenon on a copper substrate to form the microstructures of concentric circular rings. Femtosecond lasers 
can be used to produce micro-scale surface features, such as micro-holes, microgrooves, micro-cuts. Potential application of femtosecond lasers include production of medical devices, photonic devices, micro-lenses, micro-moulds, MEMS devices (Liang 2003). Low powered laser micromachining under water can be used for materials where debris formation and matter ejection is undesirable (Kruusing 1999), for example; in heatsensitive materials such as $\mathrm{HgCdTe}$; avoid cracking in $\mathrm{SiN}$ ceramic; elimination of graphite formation on diamond; avoidance of silicon layer formation on a $\mathrm{SiC}$ etc. $\mathrm{CO}_{2}$ based lasers have shown good potential over the years in high power machining of materials. Several variants of $\mathrm{CO}_{2}$ lasers have been developed. For example, TEA-CO lasers generally operates at similar wavelengths as compared to $\mathrm{RF}-\mathrm{CO}_{2}$ and other lasers, but the duration of the pulse is shorter to $1 \mu$ s as compared to; $\mathrm{RF}-\mathrm{CO}_{2}$ lasers where the pulse duration is generally in the range of $50-100 \mu \mathrm{s}$; excimer lasers $(248 \mathrm{~nm})$ at $20 \mathrm{~ns} ; 3^{\text {rd }}$ harmonic Nd:YAG lasers at 3ns (Zheng 2001).

As shown in Fig.11, the laser ablation can result in either V-shaped or U-shaped cross-sectional profile depending upon whether low fluence with higher repetition rates were used or higher fluence with lower repetition rates were used. Plasma shielding effect is also observed during laser material interactions (Bergmann 1996; Schaaf 2010). Vapor and plasma formation during laser matter interactions can be described in terms of resonant and non-resonant processes. In resonant mode, the photon's energy equals the duration of transition between two energy levels of a plasma atom, where as in nonresonant absorption, this mode is characterized by laser beam interactions with free 
charges in plasma. These interactions of photons is based on the higher mobility of electrons as compared to individual ions (Schaaf 2010).

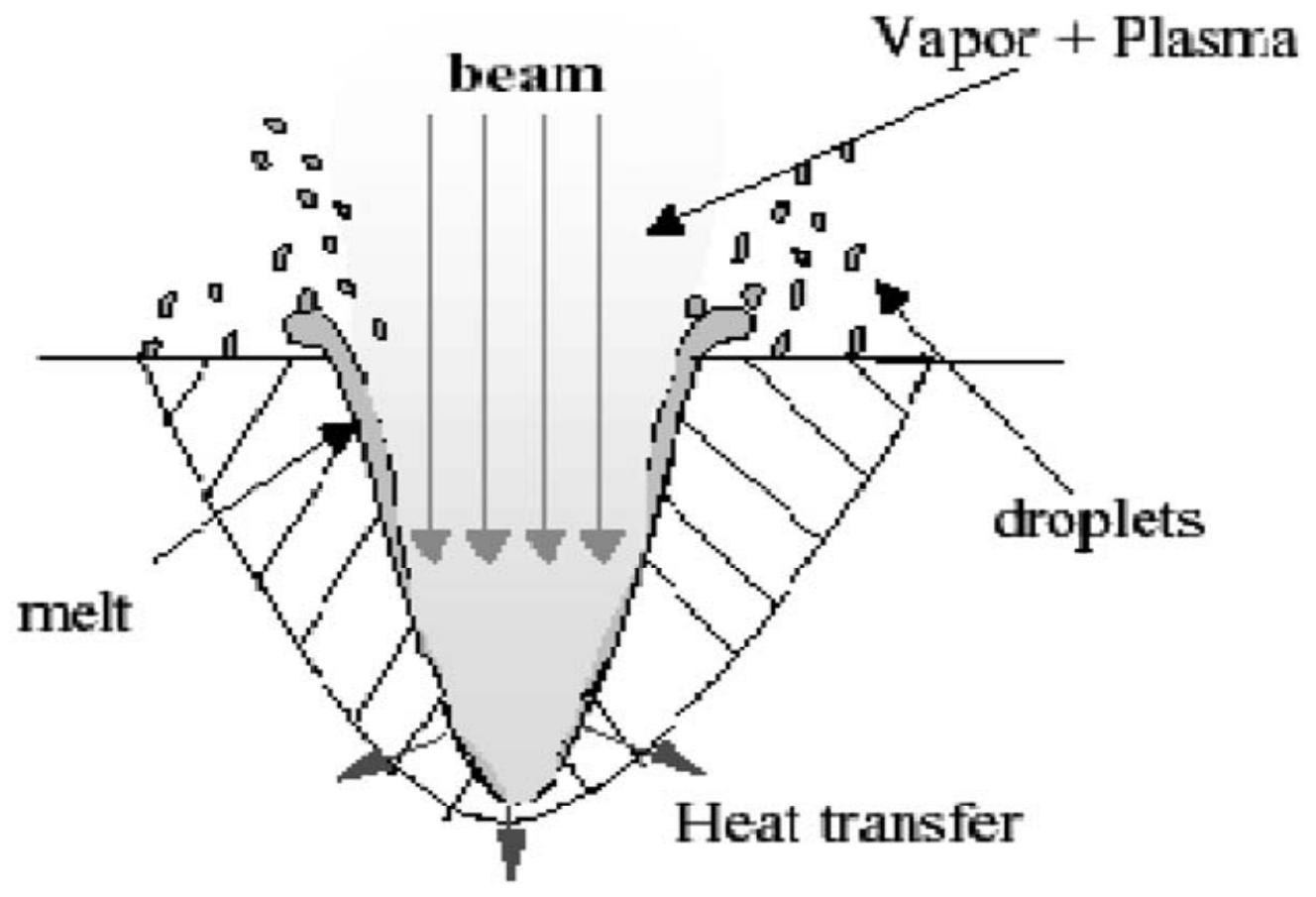

Figure 11 An illustration of plasma vapor and debris formation in-air laser ablation (Tseng 2004)

Excimer lasers are well known for their potential for being powerful manufacturing tools because of primarily two reasons; 1) Excimer lasers are reliable and well suited for industrial environments since they produce consistent output characteristics; 2) Excimer lasers can produce a variety of micromachining features with high quality. Excimer lasers find applications in virtually every field of engineering; they are used for micromachining of different materials such as polymers, ceramics, glass etc. Excimer lasers are also used for changing the structure and properties of metal oxides, manufacture of computer chips below $0.25 \mu \mathrm{m}$ size, microstructures with feature sizes 
from 1 to $100 \mu \mathrm{m}$ and refractive laser surgical applications (Basting 2002; Chen 2005). Excimer laser patterning is considered a commercially viable and is used as a highly automated serial technique (Basting 2002) (Choi 2001). Tseng et. al (2004) examined the ablation efficiency, morphology and impact of laser parameters on glass, silicon and aluminum using an UV ArF (193nm) excimer laser. They observed the ablation depth to be linearly proportional to the operating laser parameters, such as pulse number and fluence, and showed that the ablation at lower fluence and higher repetition rates tends to form a V-shaped cross-sectional profile at ablated region, whereas at higher fluence and lower repetition rates the U-shaped profile was observed. Rizvi et.al (2001) investigated the basic principles of excimer laser thin film ablative patterning by mask transfer methods and described the ablation mechanism for polymers (Rizvi 2001). Chen et. al (2005) investigated ablation behavior of borosilicate glass based single and arrayed microstructures using excimer laser (ArF-193nm). They observed excellent ablation properties well suited for fabrication of micro-devices for photonics and communication industries. Desbiens et. al (2007) characterized ablation process for silicon, PZT and SiC using an excimer laser (ArF - 193nm) and studied the dynamic interaction between the ablation and machining parameters. Excimer laser micro-patterning can also be used to induce a chemical change on surface which have widespread applications in semiconductor circuitry, sensors, printing, tissue engineering and micromechanical devices (Callewaert 2003). The ability to etch a very small amount of biological material without collateral damage has lead to a number of surgical applications (Paterson 1999). The inkjet-printer nozzle is an example of a successful commercialized product in the area of micro-scale fluidic devices (Tseng 2004). 

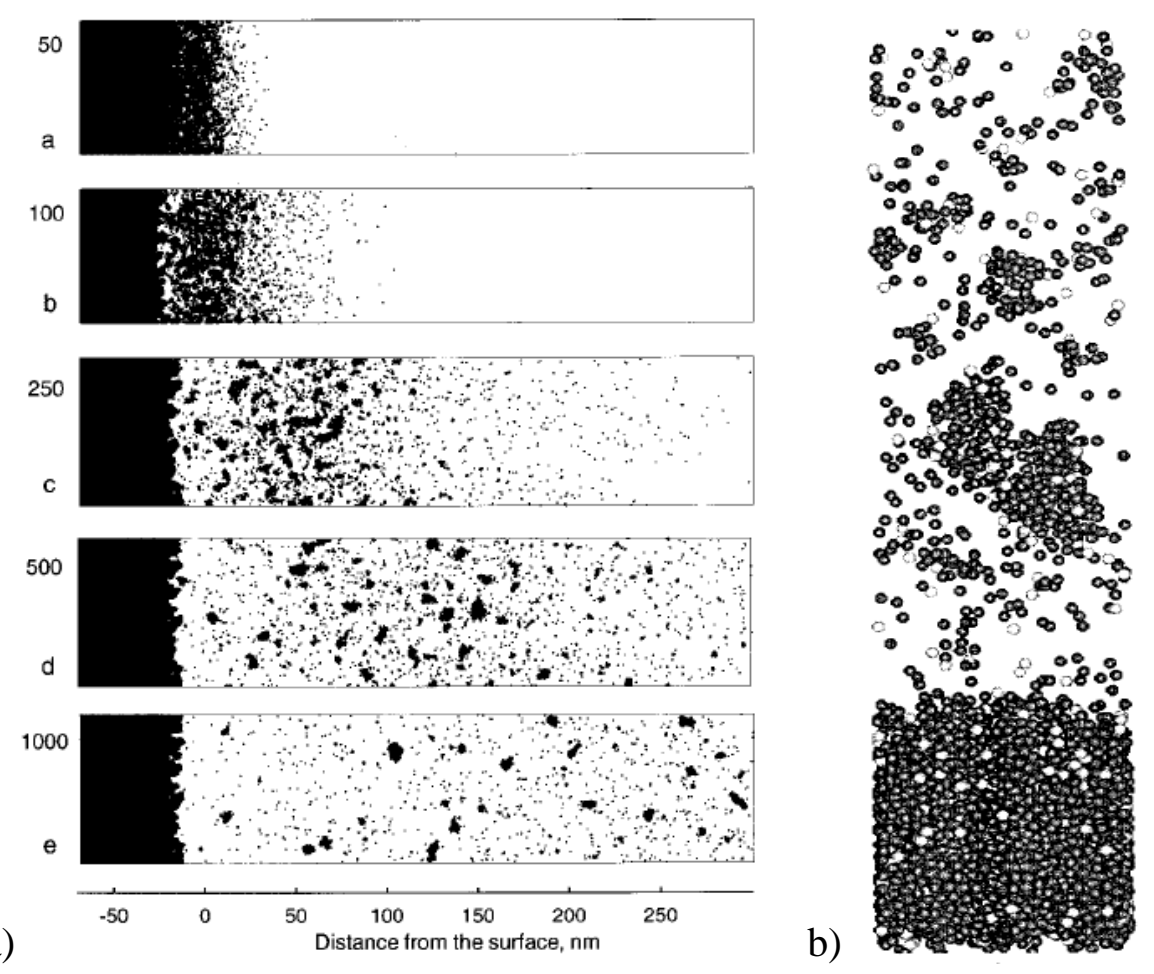

Figure 12 a) Plume development b) time development of the plume for a 2D simulation (Zhigilei 1998)

The Fig.12 (a) and Fig. 12 (b) above shows an experimental simulation model developed according to Beer's law for qualitative comparisons with experimental data. The bulk of the material is shown to the left of the figure whereas the plume expansion is shown to the right. Zhigilei et. al (1998) explained that during the plume generation, temperature rise occur at a faster rate than the mechanical relaxation of molecules thus it results in development of a pressure gradient in the direction normal to the surface. If this pressure gradient reaches a threshold value, it does a forward ejection of material for the irradiated region under laser light. 


\subsubsection{Underwater Laser Micromachining}

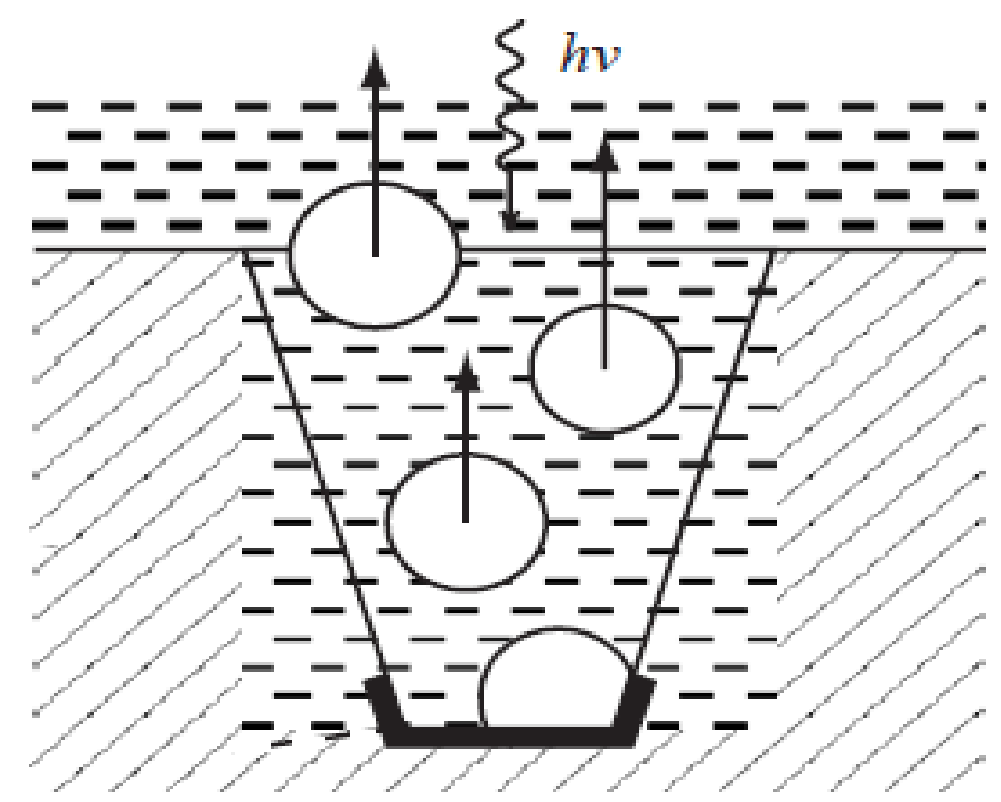

Figure 13 Micromachining underwater environment illustration (Kruusing 1999)

When laser etching is performed under liquid environment, the debris is generally carried away due to the thermal convection of the liquid or bubble formation. This effect is observed when the laser pulses are short with lower repetition rates. Kruusing et. al (2004) investigated about underwater etching and cutting by lasers and observed the material removal/response of silicon and alumina substrates along with the effect of environment on the ablation process. The primary objective of his research involved controlling heat affected zone, avoiding re-deposition of debris and irradiation of polymers under water. By lowering the laser fluence and increasing the pulse number the probability of laser shock can be reduced (Kruusing 2004). In order to maintain such situation the fluence is determined at the level where the material just starts to melt but it at least vaporize and forms plasma. Liquid environment prevents the plume development 
and thus enhances the action of laser radiation on the substrate material (Voronov 1996). Laser ablation in liquid is mostly influenced by the inherent mechanical processes that are involved as compared to under gas ablation. Higher ablation rates can be observed due to confinement of plasma and shock waves when working underwater. Presence of water under laser light cause disassociation of oxygen and encourage chemical reactions. Bubbles generally carry away the debris effectively which enhances the etching rate, but on the other hand the bubbles also scatter the laser light as well which may lower the etching rate in liquid environments (Kruusing 2004).

For working under gas environment, the laser ablation mechanism involves surface boiling, condensation, rapid ejection, micro-particle formation, nucleation of droplets, growth and furthermore generation of nano-particles. Also, the pressure buildup under the strict mechanical inertial confinement due to rapid increase of the thermal energy can lead to an overheating system whose temperature go beyond the melting point of the material, thus a larger heat affected region (HAZ) is formed (Zhigilei 1998). Zheng et al. (2001) investigated the laser micro-drilling using $\mathrm{KrF}$ excimer laser $(248 \mathrm{~nm}), 3^{\text {rd }}$ harmonic pulse Nd:YAG laser (355nm), RF-excited $\mathrm{CO}_{2}$ laser and transversely excited atmospheric (TEA) $\mathrm{CO}_{2}$ laser on PCB substrates, where they used polymide and alumina specimens to observe micro fabrication, micro-drilling behavior, laser-material interactions and effect of pulse duration on heat affected zone (HAZ) (Kruusing 2004). They concluded that in micro-drilling process the drilling speed is primarily dependent upon the laser's repetition rate and the sample's speed of movement with respect to laser beam (Zheng 2001). 
About the development of a wider heat affected zone (HAZ), Yoshihisa et. al (2009) explained that when the laser light strikes on the surface of a material the thermal conduction causes the heat profile to broaden by continuous absorption and supply of heat but there exists a temperature gradient between the peak temperature at the center and at the surrounding area. This leads to the development of pressure gradients inside the substrate (Yoshihisa 2009). By minimizing the heat affected zone (HAZ), the undesirable thermal damage can be avoided and thus small feature sizes in micro-scales can be achieved. This implies that materials in which photochemical ablation process is the dominant ablation process are the ones mostly favored for patterns and micromachining applications (Tseng 2004).
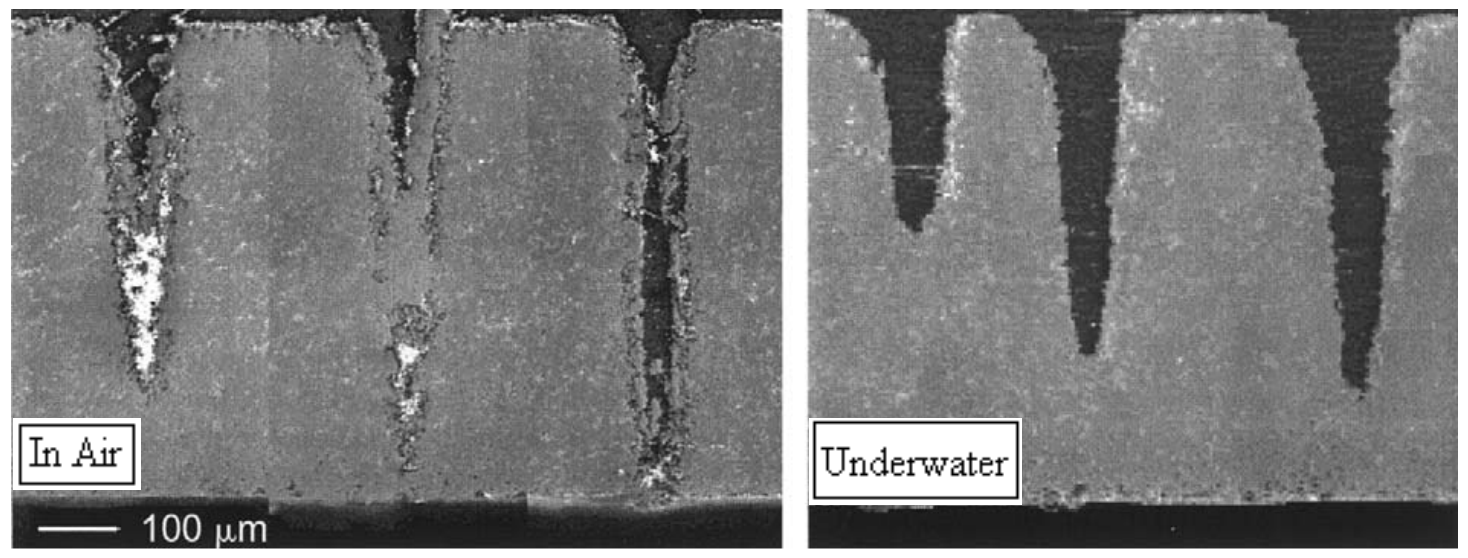

Figure 14 Laser etching of a NdFeB magnet in air and under water using Nd:YAG laser (Kruusing 1999)

As the behavior of materials to ablation process differ according to their mechanical and chemical properties, for some magnetic materials where the ablation cause large debris formation due to the nature of the materials, micromachining under 
water by short-pulsed laser provides clean grooves while machining in air results in grooves filled with molten material (Kruusing 1999; Kruusing 2004).

\subsubsection{Thin Film Micromachining}

From last two decades the excimer laser annealing of thin films is considered an alternative option for highly efficient low cost thermal annealing of thin films without much damage to underlying layers of substrate. The benefits include minimization of defect density and modification of physical and electrical properties of thin films. Miniature thin film devices can be developed with the help of excimer laser mask projection techniques (Rizvi 2001). Sedky et al. (2006) studied the possibility to treat plasma enhanced CVD using excimer laser to deposit thin films of silicon germanium at $400^{\circ} \mathrm{C}$ or lower to demonstrate high growth rate $(\sim 35 \mathrm{~nm} / \mathrm{min})$ at low temperatures with low defect density (Sedky 2006). Huang et. al (1996) reported the use of KrF excimer laser crystallized a-Si:H/a-SiN $: \mathrm{H}$ multi-quantum well (MQW) films at room temperature in an effort to produce high mobility thin film transistors for liquid crystal display technology. They improved the surface morphology, microstructures, uniformity and processing temperature of a-Si:H/a-SiN ${ }_{x}: H$ multi-quantum well (MQW) films with ability to produce nano-sized Si crystallites. Grant e.t al (2010) investigated substrate temperature effect on surface morphology of excimer laser $(\mathrm{KrF}-248 \mathrm{~nm})$ deposited $\mathrm{Yba}_{2} \mathrm{Cu}_{3} \mathrm{O}_{7} / \mathrm{CeO}_{2}$ thin films on sapphire. In thin polymer film direct patterning, only one laser shot is required to heat the surface layer, enable disruption at the bonding and ejection of hot film fragments at varying speeds (Haruna 1994; Rizvi 2001; Grant 2010). Yoshihisa et. al (2009) investigated fabrication of silicon thin films and their deposition 
and observed possibility of dry etching and laser thermal lithography for materials with high gasification temperatures such as $\mathrm{SiO}_{2}$ and sapphire. Ryoichi et. al (2003)reported precise location control of $\mathrm{Si}$ individual grains and assessed performance in singlecrystalline thin film transistors by excimer laser induced crystallization. Their method induced local structural modifications in underlying materials using a conventional photolithography, which have widespread applications in biomedical field (Ryoichi 2003). Papantonkis et. al (2006) investigated thin film deposition of polytetrafluoroethylene (PTFE, trade name Teflon $®)$ with the help of a process called ultraviolet pulsed laser deposition (UV-PLD) and explored the effectiveness of a resonant infrared laser ablation process to produce high-quality films of a various families of polymers. They deposited thin films with the help of a Free-Electron Laser (FEL) on microscale structures which showed good adhesion, excellent smoothness and high degree of conformability to the substrate structures. The other primary objective of the research was to measure the deposition-rate as a function of laser intensity or fluence. In general, pulse energy of $1 \mathrm{~J} / \mathrm{cm}^{2}$ is enough to remove the polymer thin films of thicknesses around 100nm, similarly for thin film thicknesses around 10nm, only $10 \mathrm{~mJ} / \mathrm{cm}^{2}$ energy is required (Rizvi 2001; Yoshihisa 2009). Haruna et. al (1994) utilized an excimer laser (ArF-193nm) to demonstrate epitaxial growth of $\mathrm{LiNbO}_{3}$ films to form electro-optic thin film waveguides on sapphire and $\mathrm{LiTaO}_{3}$ c-plates, which can largely benefit development of waveguide devices such as light reflectors, interferometric modulators etc. Shen et. al (1996) demonstrated that the laser focus position adjustable in Z-direction can be used to process 3D microstructures under laser influence. They used small increments of laser focus lowered at $3 \mu \mathrm{m} /$ pass to develop a 12 to $18 \mu \mathrm{m}$ deep 3D 
microstructure with material removal rate of $600 \mu \mathrm{m}^{3} / \mathrm{s}$. Tungsten and silicon were successfully deposited with the help of different gases $\mathrm{Ar}, \mathrm{H}_{2}, \mathrm{WF}_{6}$ and $\mathrm{SiH}_{4}$. Low cost manufacture of complex sensors devices, flexible printed circuits, electrical interconnection circuits, miniaturization and rapid prototyping can be achieved using direct laser patterning of thin films (Shen 1996; Rizvi 2001). Paterson et. al (1999) investigated excimer laser ablation of microstructures of PMMA and polyimide and proposed a numerical model to predict the wall angles of trench structures ablated with partially coherent illumination. For explanation of the laser ablation process, researchers have worked on molecular dynamics model where a group of atoms are treated as a unit as compared to treating an atom as a single entity (Garrison 1984; Garrison 1985; Paterson 1999). Attempts have been also made in observing the variation of wall angle with fluence with a numerical model (Kahlert 1992; Paterson 1999). The simulation model about ablation developed by researchers can help translate microscopic observations into mesoscopic characteristics such as local temperature, energy distributions, pressure and particle motion (Zhigilei 1998).

\subsection{Excimer Laser 2D/3D Patterning}

For development of functional micro devices, a considerable research effort has been devoted to laser patterning of two-dimensional (2D) or three-dimensional (3D) microstructures using lasers. Excimer laser ablation is a powerful tool for the generation of high precision microstructures. For example; in micro-optics, mechanical precision alignment with wave guiding structures for integrated optical elements; and in microfluidic domain, filters and micro-meshes for particle separation in flow channels or 
mixing of fluids. All these uses of excimer laser ablation patterning are possible due to high precision and resolution achievable. Ricciardi et. al (1996) used excimer laser to engrave laser symbols on polystyrene and polycarbonate (PC) for high speed production of computer keyboards and investigated color change for optimized PC/ABS mixture containing $0.5 \%$ titanium oxide with laser irradiation. Dowling et al. (2001) used an excimer laser $(\mathrm{KrF}-248 \mathrm{~nm})$ to assess the relative performance of patterning TiN films from metal sacrificial layers and reported etching behavior, edge quality, film delamination and layer selectivity. They used silicon as a substrate with sputtered $\mathrm{Cr}$ and $\mathrm{Cu}$ metal sacrificial layers in partially filtered arc deposition system at $150^{\circ} \mathrm{C}$ to directly pattern the TiN films on silicon. Choi et. al (2001) presented a simple fabrication method to produce 3D bioMEMS devices for a high aspect ratio micro-electrical structure using an excimer laser. Callewaert et. al (2003) investigated excimer laser induced patterning of polymeric surfaces and used an excimer laser (ArF-193nm, KrF-248nm) for surface modification of polyurethane and developed a new micro-patterning procedure. Choi et al.(2004) also proposed a similar 3D micro-machining method using excimer laser called Hole Area Modulation (HAM) in which fabrication is done using excimer laser with movement of workpiece with respect to laser beam to realize different types of $3 \mathrm{D}$ microstructures. They also proposed a numerical model based on HAM for optimum laser ablation parameters to successfully create micro-lens. Barun et. al (1998) employed a (KrF-248nm) excimer laser and developed a contour mask technique to create microprismatic structures where polymer sample was made to move with respect to mask for realizing a rapid replication process. They used a planarizing layer on top of substrate to eliminate debris formation and redeposition problems with polymer ablation. Zimmer et. 
al (1996) studied the fabrication of analogous 3D microstructures using $\mathrm{KrF}$ (248nm) excimer laser with mask projection technique and produced some binary geometrical micro-features like micro-holes, micro-cuts etc. Lee et. al (2005) proposed a new method for fabrication of axially symmetric 3D microstructures based on a $\mathrm{KrF}(248 \mathrm{~nm})$ excimer laser. They used a novel mask contour scanning technique for obtaining high quality microstructures $(\sim 500 \mu \mathrm{m}$ and avg surface roughness less than 10nm) on Polycarbonate (PC) samples without compromising the machining speed.

Fig.15 illustrates the principles of direct patterning of thin films by pulsed excimer laser ablation of polymer where each pulse from the laser removes a small decomposed and ejected layer. The material removal was observed to be in the range of 0.1 to $0.3 \mu \mathrm{m}$ with pulse energies in the range of 0.1 to $1 \mathrm{~J} / \mathrm{cm}^{2}$ (Rizvi 2001). Arnold et. al (1995) developed a multistep pattern replication process comprising of excimer laser (ArF -193nm) micromachining, inverse replication by electroplating process and second inverse replication by injection molding process. Ablation was carried out on PMMA microstructures with high structural fidelity and low cost production suitable for large scale manufacturing of moulds. 


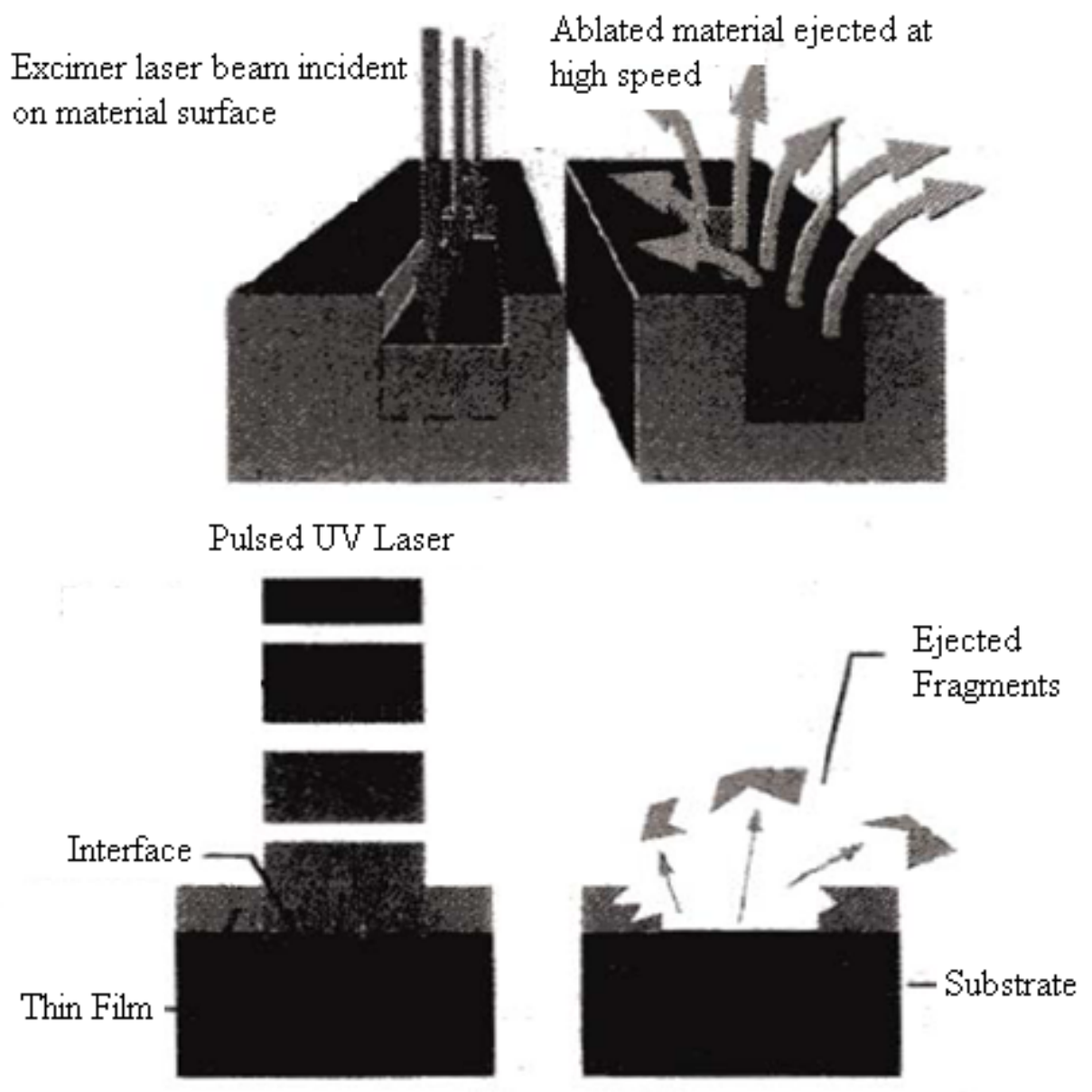

Figure 15 Excimer laser interaction with a)thick polymer film and b)thin metal film (Rizvi 2001) 


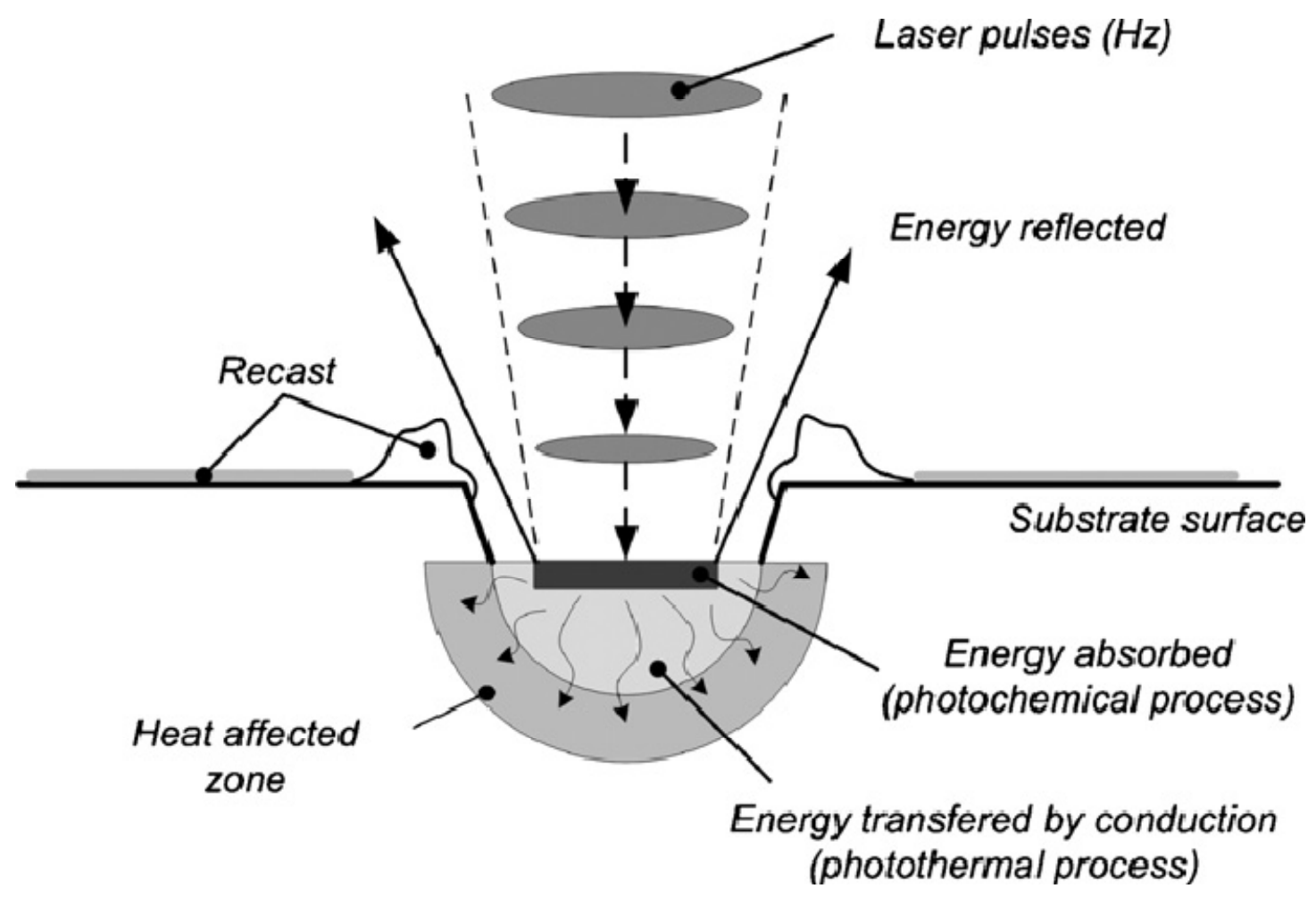

Figure 16 Laser-matter interaction of a pulsed laser beam (Desbiens 2007)

As laser ablation process is, by nature, a very complex and has been established as a research interest topic throughout scientific world. It is also due to the fact that it involve diverse processes such as laser excitation of absorbing molecules, energy transfer from excited molecules into transitional and internal modes of the solid, material ejection and disintegration, and consequentially plume development (Zhigilei 1998). Very few quantitative results could be found in the literature about the material interaction with laser parameters involved for micromachining using a workpiece dragging techniques (workpiece displaces with respect to laser beam), most of the researchers have focused on static machining practice, i.e. micro-holes, micro-patterns etc (Desbiens 2007). Based 
on a recent study, the laser ablation is believed to be a collective effect of matter, rather than a sequence of collisions between molecules or atoms (Zhigilei 1998). It is characterized by the pulse-by-pulse removal of small amounts of material from the illuminate region of the target with least damage to the surrounding or underlying surface (Paterson 1999). Water is sometimes used as the ambient environment due to the fact that it eliminates the debris re-deposition over the substrate (Kruusing 2004). Decrease in etching rate for underwater micromachining may happen due to lower number of pulses, accumulation of debris, destruction of oxide layer and reduction in transparency of the liquid (water) (Kruusing 1999). Similar differences have been observed for comparisons of underwater, gases and air (Kruusing 2004). Zhigilei et. al (1998) investigated about dependence of shape of ablation crater on irradiation regime and concluded laser ablation to be a collective process, wherein particles of interest involve several hundreds of atoms or molecules which participate in the ablation and plume development phenomenon. They also stated significant involvement of time scale or pulse duration in plume development where the particle removal process occurs on longer time scales or longer pulse durations. Ejection of the matter is affected due to overheating of the material where phase explosion or explosive boiling takes place in which the matrix decomposes into a gaseous mixture of cluster of molecules (Zhigilei 1998). The rapid ejection provides a faster means of cooling of the ejected matter and the irradiated region. It is also observed that the pressure driven ablation leads to different ejection conditions for molecules depending on their original depth inside the substrate (Zhigilei 1998). 
The ablation phenomenon in laser micromachining could be observed in three forms; photothermal, photochemical or combination of both. In photothermal ablation process, the absorbed laser energy is converted to lattice vibrational thermal energy to cause melting and vaporization of the material (Tseng 2004). In photochemical ablation process, the removal of material is usually proceeds by direct breakage of atomic bonds as soon as energy is absorbed by the surface of material (Tseng 2004). The photochemical ablation process is mainly dependent upon the absorption coefficient $(\alpha)$ of the material, where the maximum depth of penetration is governed by the reciprocal of the absorption coefficient $(\alpha)$. Excimer lasrs (ArF, $\mathrm{KrF}$ etc) exhibit photothermal ablation phenomenon even for very small duration pulses ( $>5 \mathrm{~ns})$ as compared to femtosecond lasers where the thermal effects are considerably small (Desbiens 2007). Photofragementation phenomenon occurs when an excited molecule reacts photochemically with the molecules and forms material fragments (Zhigilei 1998). Beer's law can be used to explain absorption probability and reproduce the exponential attenuation of the laser light with penetration depth due to laser irradiation (Zhigilei 1998). Equation 4.1 (Tseng 2004) gives Beer's law:

$$
\Delta d=\ln (F i / F t) / \alpha
$$

Where, $\Delta \mathrm{d}$ is the ablation depth/pulse, $\alpha$ is the absorption coefficient, Fi and Ft are the incident and threshold fluences.

A higher ablation coefficient results in lower ablation threshold fluence, for example in thin films deposition and material removal the polymer layers on top of delicate material can be processed without damage to the underlying structure (Rumbsy 
1997). It has been observed that linear absorption and conformance with Beer's law is obeyed by most materials but only over a limited range of fluences (Paterson 1999). For example, in RF-excited $\mathrm{CO}_{2}$ lasers, the absorption depths are much deeper as compared to FEL or UV lasers (Zheng 2001). Whereas the drilling rates of a few tens of microns/pulse are possible. Dyer et. al (1996) estimated the ablation coefficient for glass using probe-beam deflection technique and proposed following Equation 4.2 (Dyer 1996):

$$
\alpha=S K C /[(1-R) E]
$$

Where, $\mathrm{R}$ is the surface reflection coefficient, $\mathrm{C}$ is the volume specific heat, $\mathrm{E}$ is the excimer laser fluence, $\mathrm{S}$ is the deflection signal voltage and $\mathrm{K}$ is a constant.

Equation 4.3 provides the relation of thermal diffusion length $(\mathrm{L})$ as a ratio of thermal conductivity of material (D), heat capacity and the pulse width (t) (Zheng 2001).

$$
L=(D t)^{1 / 2}
$$

Equation 4.4 gives the total yield of ablated material Vs. energy deposited per unit volume (E/V) (Papantonakis 2006):

$$
\text { Yield } \propto\left(\frac{E}{V}\right) \cong F_{L} \alpha(\omega, I) \cong I_{o} \tau_{L}\left[\propto_{\circ}(\omega)+\beta I\right]
$$

Where, $\mathrm{F}$ is the laser fluence, $\propto$ is the absorption coefficient comprising linear $\propto_{\mathrm{o}}$ and non-linear $\beta$ terms, $\omega$ and $I$ are the laser frequency and intensity, respectively. 
In micromachining of polymers, the excimer laser ablation of polymer materials has been investigated by various researchers in last few decades (Srinivasan 1984; Sutcliffe 1986; Kuper 1987; Bruan 1989; Rumsby 1991; Gower 1992). It has been observed that the interaction of pulsed high repetition excimer laser with polymer surface leads to ablative photo-decomposition which results basically due to etching of polymer surface and explosive ejection of decomposition products at supersonic velocities (Callewaert 2003). Ricciardi et. al (1996) also observed the change of color due to photochemical reactions on polymer surface due to high photon energy but with negligible thermal effects. The polymer ablation using mask projection has been observed to be having an important ability to perform micromachining with high accuracy, high aspect ratios and considerably deeper than depth of focus of the imaging optics of the laser (Paterson 1999). Papantonakis et. al (2006) observed for Free-Electron lasers (FEL) with polymer-laser material interactions that the laser can be programmed to excite specific molecular degrees of freedom with mid-infrared photons which lacks sufficient energy to initiate any damaging photochemical reactions.

In semiconductor materials, Zheng et. al (2001) observed the laser micro-drilling performance of $\mathrm{KrF}$ excimer laser (248nm), $3^{\text {rd }}$ harmonic pulse Nd:YAG laser (355nm), RF-excited $\mathrm{CO}_{2}$ laser and transversely excited atmospheric (TEA) $\mathrm{CO}_{2}$ laser on PCB substrates which demonstrated that most PCB materials exhibit finite absorption characteristics with respect to laser's wavelength, therefore only certain laser wavelengths are compatible with a particular PCB material. Shen et. al (1996) observed that silicon can be etched by chlorine in a micro-reaction controlled by a laser where the 
etching process is directly controlled by the amount of laser power, speed of scanning, gas pressure and the spot size of the laser beam.

For common metals, Rumsby et. al (1997) reported that ablation rates depends on the thickness of metal layer varying from complete metal removal with one laser shot for some thin films (up to $100 \mathrm{~nm}$ ) to multiple 10 s of laser shots for films up to $30 \mu \mathrm{m}$ thick. Each pulse of laser removes a fraction of film thickness. In optics, a supercontinuum is (SC) is formed when a collection of nonlinear processes act together upon a pump beam in order to cause severe spectral broadening of the original pump beam. Lin et. al (2006) used a Ti:sapphire laser $(830 \mathrm{~nm})$ on photonic crystal fibers (PCF) with average laser power of $350 \mathrm{~mW}$ to observe the influence of laser power and ambient temperatures on the supercontinuum (SC) generation. They confirmed that the threhold pump power for SC build-up depends on ambient temperature of fibers. The laser fluence values for metals and ceramics are relatively higher than losely structured materials thus requires photothermal mode of ablation (Tseng 2004). The steel 304 AISI material is observed to exhibit material hardening due to the fact that etching underwater causes laser shock because the etching rate for first few pulses is considerably higher than later pulses (Kruusing 2004). For $\mathrm{Al}_{2} \mathrm{O}_{3}$ and $\mathrm{MgO}$, the higher etching rate results in underwater laser machining as compared to air., however, no satisfactory explanations were observed in the literature that why $\mathrm{Al}_{2} \mathrm{O}_{3}$ and $\mathrm{MgO}$ shows exceptional ablation/etching behavior (Kruusing 2004). For $\mathrm{ZrO}_{2}, \mathrm{SiC}, \mathrm{Si} 3 \mathrm{~N} 4$, glass and stainless steel the etching rate is lower in underwater as compared to air (Kruusing 2004). For a fluence of $20 \mathrm{~J} / \mathrm{cm}^{2}$, silicon ( $\mathrm{Si}$ ) shows fluctuation in etch rate, melting and poor control 
over machined geometry (Desbiens 2007). For a fluence of $2.4 \mathrm{~J} / \mathrm{cm}^{2}$ at $10 \mathrm{~Hz}$ with 150 250 pulses, the ablation depth of aluminum or silicon is less than that of glass (Tseng 2004). Thermal conductivity of the material being ablated is also very important in determination of ablation efficiency or effectiveness. For example, aluminum have thermal conductivity $(238 \mathrm{~W} / \mathrm{mK})$ at $660^{\circ} \mathrm{C}$ as compared to silicon $(157 \mathrm{~W} / \mathrm{mK})$ at $\left(1410^{\circ} \mathrm{C}\right)$ and with glass $(1.1 \mathrm{~W} / \mathrm{mK})$ is higher (Tseng 2004). Thus lesser ablation depth is expected due to rapid dissipation of heat inside the material which leads to irregular heat affected zone and thermal profile (Tseng 2004). The laser threshold of copper with $\mathrm{KrF}$ excimer laser was observed at $3 \mathrm{~J} / \mathrm{cm}^{2}$ (Zheng 2001). Unlike polymers, the micro-drilling of copper requires higher input energy.

In glass and dielectric materials, short UV wavelength lasers such as $\mathrm{KrF}$ (248 nm) lasers are well coupled and absorbed with common dielectric materials (Zheng 2001). Whereas the laser light of wavelengths around $355 \mathrm{~nm}$ are very reactive with the dielectric materials. For glass fiber materials, the quality of ablated or laser processed surface depends upon the thermal and mechanical properties of the material along with laser processing parameters. Also, the interaction of laser beam with the glass/epoxy matrix depends on sevral factors such as beam coupling, thermal conduction, melting and vaporization (Zheng 2001). Zheng et. al (2001) observed that for glass fiber materials it is more important to ablate and remove material quickly instead of using a beam with lower fluence due to the fact that less heating would result with shorter pulses with high pulse energies which would essentially eliminate unwanted thermal transfer process to heat surroundings. $\mathrm{CO}_{2}$ lasers emit a medium wavelength $(10.6 \mu \mathrm{m})$ in infrared regions 
where absorption in dielectric materials is higher up to $>90 \%$. Therefore, when the etch rate is slow then hundreds of pulses are required to etch a thick dielectric layer $(120 \mu \mathrm{m})$ (Zheng 2001). The required energy density for complete micro-drilling a particular material bye laser varies with respect to its thickness.

Glass as compared to other materials like aluminum and silicon have very low thermal conductivity $(1.1 \mathrm{~W} / \mathrm{mK})$ (Tseng 2004), thus the ablation efficiency is expected to be higher as compared to aluminum or silicon. Following table highlights important properties of the glass, aluminum and silicon:

Table 6 Thermo-physical properties of glass, aluminum, and silicon (Tseng 2004)

\begin{tabular}{lllll}
\hline Properties & & Glass & Aluminum & Silicon \\
\hline Density & $\rho_{\mathrm{s}}\left(\mathrm{kg} / \mathrm{m}^{3}\right)^{\mathrm{b}}$ & 2230 & 2700 & 2330 \\
& $\rho_{\mathrm{l}}\left(\mathrm{kg} / \mathrm{m}^{3}\right)$ & 2030 & 2385 & 2130 \\
Heat Capacity & $C_{\mathrm{s}}(\mathrm{J} / \mathrm{kg} \mathrm{K})$ & 712 & 917 & 716 \\
& $C_{\mathrm{l}}(\mathrm{J} / \mathrm{kg} \mathrm{K})$ & 1200 & 1080 & 1200 \\
Thermal Conductivity & $k_{\mathrm{s}}(\mathrm{W} / \mathrm{mK})$ & 1.10 & 238 & 157 \\
& $k_{\mathrm{l}}(\mathrm{W} / \mathrm{mK})$ & 2.87 & 100 & 30 \\
Melting Temperature & $T_{\mathrm{m}}\left({ }^{\circ} \mathrm{C}\right)$ & 820 & 660 & 1410 \\
Absorptivity & $A_{\mathrm{s}}$ & 0.8 & 0.059 & 0.02 \\
& $A_{\mathrm{l}}$ & 0.8 & 0.064 & 0.05 \\
\hline
\end{tabular}

For materials in Table 6, the ablation depth showed a linear relationship with increase in fluence, this observation is also consistent with results of Kautek et. al (1996). Glass materials are very fast in becoming the material of choice in photonics, communication and MEMS device industries (Chen 2005). Excimer lasers allow careful processing of these high precision glass structures without affecting their strength and properties (Chen 2005). For glass, the laser ablation process is usually photothermal which takes place because the laser energy is unable to heat the glass to its melting 
temperature at lower fluences, thus glass requires higher repetition rates to overcome this problem (Tseng 2004). Plasma shield around ablation region of glass can also form due to continuous bombardment by laser beam pulses by temperature rise of ionized vapor. However, the life of the plasma is generally very short on the order of femtoseconds (Tseng 2004).

At higher laser fluences, the dielectric materials respond with a plasma shielding effect in which the surrounding gas is partially ionized and maintains its speed and direction towards laser beam and thus protects the surface from laser radiation exposure (Zheng 2001). One of the major defects in lasers is recast and molten debris formation. The presence of molten phase can lead to molten droplets and debris re-deposition and solidification on the surface of the crater (Chen 2005). The shape and form of the molten droplets after solidification hints at significant difference in solidification time scales. In case of glass ablation, the heat of molten droplets on the surface after being re-deposited does not get transferred into surface fast enough to melt the underlying surface (Chen 2005). Thus molten droplets solidify to form flakes or debris. For thick and thin films removal, thick dielectric materials show higher ablation rates and lower ablation threshold fluences as compared to thick metal films (Rumbsy 1997). For example, with a

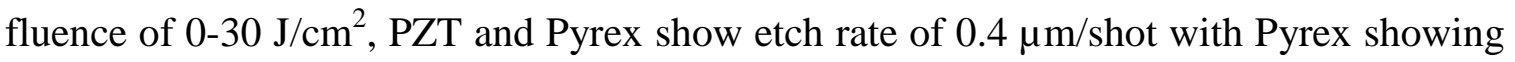
cracking above $10 \mathrm{~J} / \mathrm{cm}^{2}$ (Desbiens 2007). For diamond, the laser etching involves no qualitative differences independent of the ambient environment (Kruusing 2004). 


\subsection{Micromachining of Polymers}

Since polymers usually have relatively lower laser fluence thresholds, the photochemical ablation dominates the polymer's ablation process. Therefore, for materials with higher laser fluence thresholds, such as ceramics, metals, the photothermal process dominates the ablation process (Tseng 2004). Polymers are best suited for microstructuring due to low ablation thresholds, smooth etching behavior, rapid ablation rates with minimum number of pulses and modest fluence values (Zimmer 1996). Photothermal mode of ablation is observed when the material is ablated both in vapor and liquid phases (Chen 2005). For laser irradiation (Xe, $308 \mathrm{~nm}$ ) of PRFE polymers under water, increase in surface wetting and adhesion strength was reported due to replacement of fluorine atoms in PFTE chains and addition of $\mathrm{H} 2 \mathrm{O}$ molecules by a photochemical reaction (Kruusing 2004). For a single layer polyimide substrate, upon subjected to laser micro-drilling operation the etch rate of $0.12 \mu \mathrm{m} /$ pulse was observed with a fluence of $400 \mathrm{~m} \mathrm{~J} / \mathrm{cm}^{2}$ (Zheng 2001). For polyimide underwater laser etching, the etching rate remains same as compared to air when the laser fluence is below $20 \mathrm{~J} / \mathrm{cm}^{2}$ (Kruusing 2004). For polyimide, UV lasers are generally used with preference due to the fact that they produce the highest resolution with low thermal damage to the substrates (Zheng 2001). For annealing applications in polyimide excimer laser ablation, the invariance of the threshold fluence by annealing temperature occurs due to photochemical nature of the ablation process (Chen 2005). Which mean that even after changing the initial annealing condition, no noticeable effect on threshold fluence occurs. 
The other polymers such as PMMA, melamine, polystyrene, urea and ketone show very small or little ablation at $100 \mathrm{~mJ} / \mathrm{cm}^{2}$ for $\mathrm{KrF}$ excimer laser (Suzuki 1998). PMMA is known to exhibit an incubation effect, where the previous laser pulses affect the current ablation etch rate (Paterson 1999).

\subsubsection{Polyurethane (PU)}

Technically, polyurethane combines the best properties of both plastic and rubber. It is a thermoplastic polymer with low melting temperature $\left(177-232^{\circ} \mathrm{C}\right)$, specific gravity $\left(1.19 \mathrm{~kg} / \mathrm{m}^{3}\right.$ at $\left.20^{\circ} \mathrm{C}\right)$, water solubility $\left(<1 \mathrm{mg} / \mathrm{L}\right.$ at $\left.25^{\circ} \mathrm{C}\right)$ (NICNAS 2000). Polyurethanes are also used in a number of medical device applications such as short term implants, injection molded devices and artificial organs. Mackay et. al (1995) developed a new trileaflet heart valve prosthesis based on polyurethane polymer. When working with UV lasers, polyurethane have good ablation properties in terms of no debris formation and cavities with high dimensional quality (sharp edges with low surface roughness) (Callewaert 2003). Within the field of biomaterials, polyurethane is considered as one of the suitable material to have good biocompatibility, extensive structure and property diversity (Mackay 1995; Callewaert 2003). Surface modification is an effective means to alter biological interactions with the surface of the material, polymers like polyurethane, polyethleneoxide, and polycrylamide have played an important role in the development of medical devices ranging from tubular vascular prostheses to artificial hearts (Mackay 1995; Callewaert 2003). Masubuchi et. al (2001) studied the ablation dynamics of polyurethane films using a $\mathrm{KrF}(248 \mathrm{~nm})$ excimer laser and observed that polyurethane have low ablation threshold $\left(40 \mathrm{~mJ} / \mathrm{cm}^{2}\right)$ with high etching rate (etch depth of $0.5 \mu \mathrm{m}$ at 1 
$\mathrm{J} / \mathrm{cm}^{2}$ ), they concluded that, during ablation, material decomposition predominantly occurs via photochemical pathway.

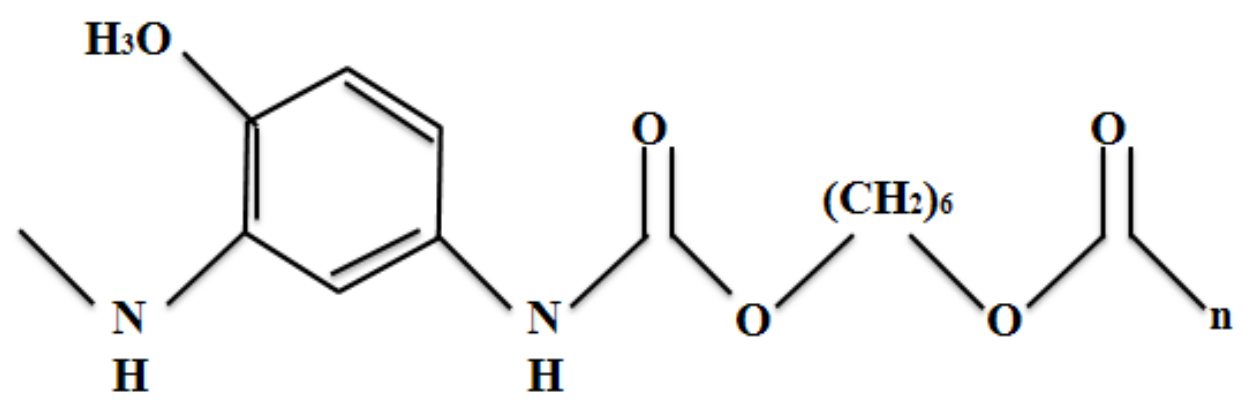

Figure 17 Chemical structure of polyurethane (Masubuchi 2001)

Suzuki et. al (1998) also studied the ablation mechanism of various polymer resist materials (phenol, novolac, PMMA, polyimide, polyurethane, urea, melamine, polystyrene and ketone) and reported data on the absorption coefficient, ablation rate and uniformity of surface ablation using an excimer laser $(248 \mathrm{~nm})$ at $100 \mathrm{~mJ} / \mathrm{cm}^{2}$. 
Fig. 18 shows the behavior of absorption coefficient and the ablation rate (Suzuki 1998):

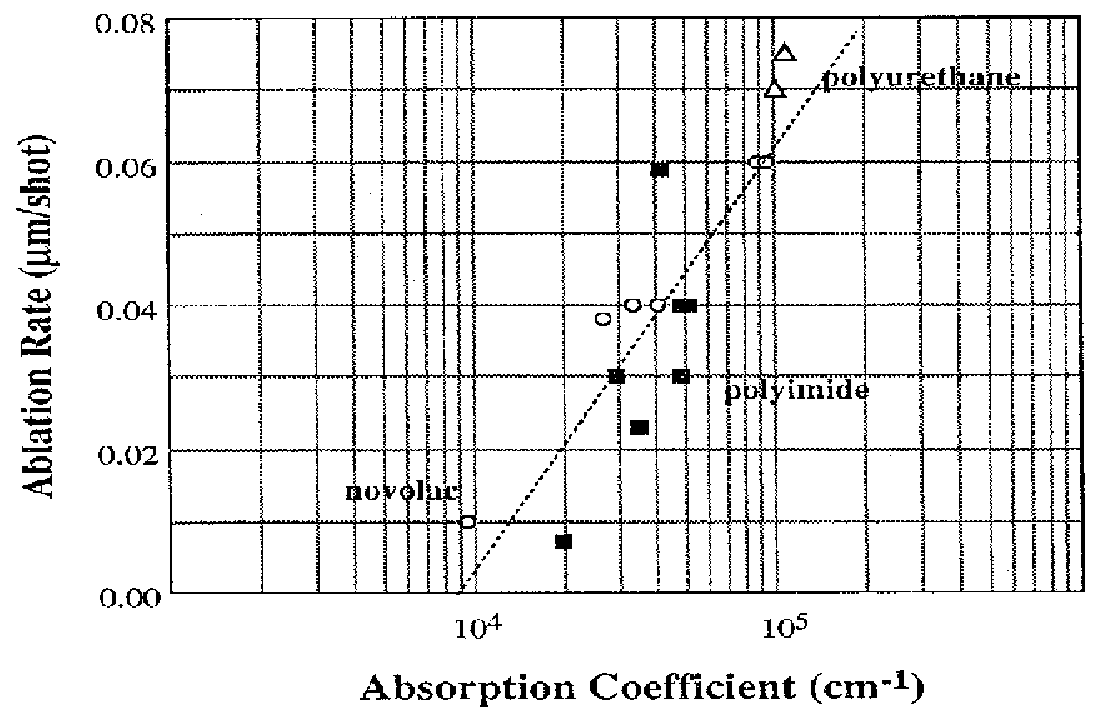

Figure 18 Important results about correlation between absorption coefficient and ablation rate of polymers (Suzuki 1998)

As seen from Fig. 18 that the materials with absorption coefficient less than $1 \times 10_{4} \mathrm{~cm}^{-1}$ show very little or no ablation for higher fluences $\left(>100 \mathrm{~mJ} / \mathrm{cm}^{2}\right)$. This phenomenon is dependent upon variation of primary/secondary structure of the polymers. A primary structure of a polymer is a combination of its chemical characteristics and its constituents, for example, in polyurethane constituents such as poly-isocyanate, diphenylmethane diisocyanate and chains of polyol exists. These constituents control the ablation behavior of polyurethane. The difference in type of structure, chain or ring structure, also plays an important role in ablation dynamics and plume generation (Suzuki 1998). The research effort in developing composite polymers has lead polyurethane to be the polymer of choice because it offers ability to form polyurethane networks where constituent polymers are chemically distinct (Aldesulu 1982). 


\subsection{MEMS Devices and Patterns in Laser Micromachining}

Today many MEMS applications have expanded exponentially and progressively with more complex structures and miniaturization is an important trend in biotechnology, microelectronics, telecommunication and medical technology (Choi 2004). New upcoming generations of MEMS devices require layered fabrication of 3D structure consisting of several thick layers $(>5 \mu \mathrm{m})$ made up of glass, silicon and ceramics (Desbiens 2007). Currently, the modern materials in MEMS devices are limited due to fabrication process limitations. MEMS devices and hard disks are generally coated with "Teflon-like" materials which require micromachining and micro-processing to obtain the required high surface-to-volume ratio, a prerequisite for micro-electromechanical systems (MEMS) (Papantonakis 2006). For micromachining patterns with the help of laser micro-drilling, the process of laser micro-drilling can be a serial process with one hole drilled at a time or a mask imaging process where many holes can be drilled simultaneously (Zheng 2001). At the least complexity of patterning, periodic arrays of micro-holes can be obtained by controlling X-Y translational stages while simultaneously being in sync with laser output. Pethig et. al (1998) performed a multilayer fabrication of micro-electrode structures with very small $(>10 \mu \mathrm{m})$ size features using a $\mathrm{KrF}$ excimer laser (248nm). Their work was directed towards developing a biofactory-on-a-chip (BFC)

device capable of performing a wide range of complex diagnostic tasks in a single, miniaturized and economical package. 


\subsubsection{Mask projection}

Many researchers in MEMS device fabrication research have used mask projection technique to realize a variety of $2 \mathrm{D} / 3 \mathrm{D}$ microstructures. The laser projection optics system can be used to project a mask that contains a desired pattern to the surface of the substrate (Zheng 2001). Mask projection technique is widely employed to produce 2D and 3D microstructures by various researchers (Zimmer 1996; Rumbsy 1997; Rizvi 2001; Zheng 2001; William 2004). A large number of holes in an array can be drilled simultaneously using the mask projection technique illustrated in figure above. For a limited area, the number of holes are usually limited by the hole size and the spacing between the holes, whereas the processing time is generally very short to a fraction of a second (Zheng 2001).

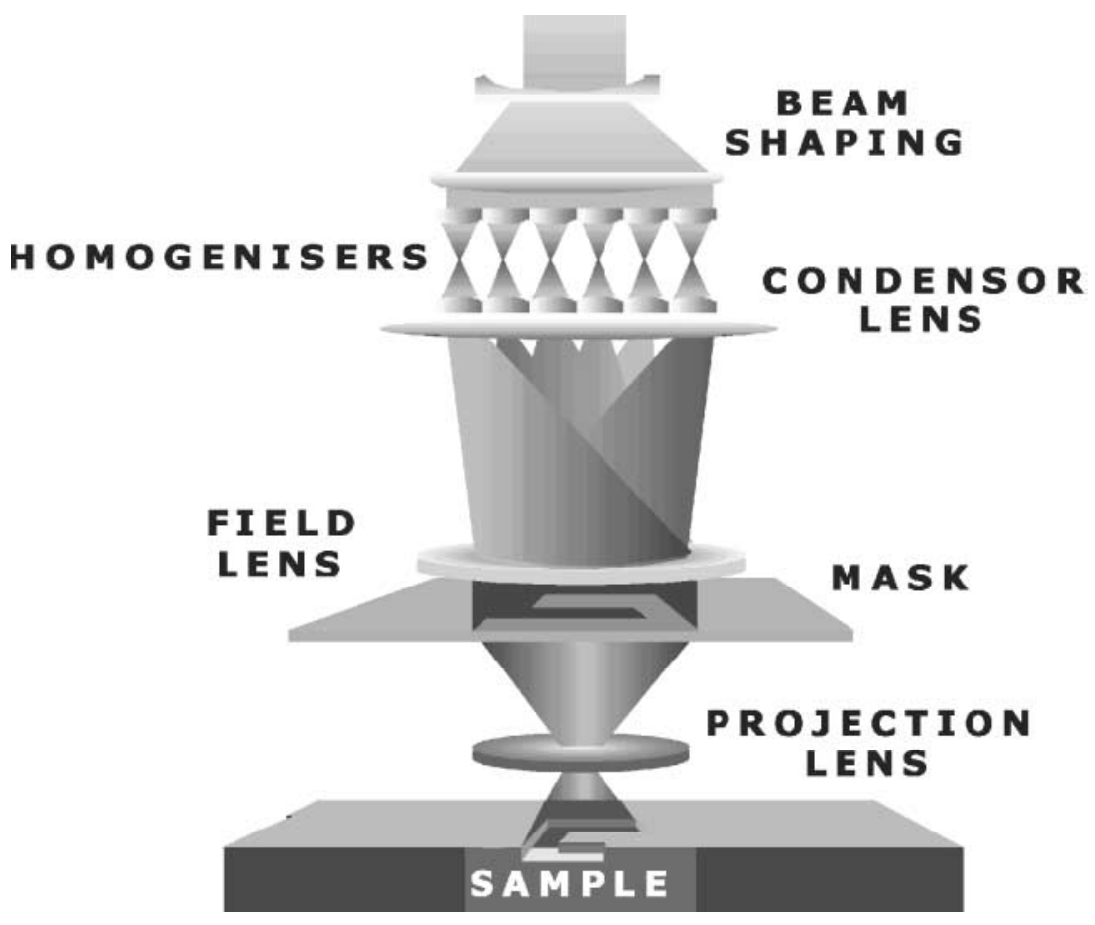

Figure 19 Mask projection technique illustration (Zheng 2001) 
Rumsby et. al (1997) investigated polymer ablation for high density excimer laser 2D patterning of polymer thick or sputtered thin films using mask projection technique for fabrication of multi-chip module interconnection, miniature sensor elements and miniature flexible printed circuits. Choi et. al (2004) used mask projection technique with a new kind of mask positioning model, called hole area modulation (HMA), where sequential holes with different and distributed diameters are used to form 3D cavity because the machining depth is converted to the sizes of the holes on the mask. Rizvi et al. (2001) developed techniques to manufacture miniature thin film devices by excimer lasers for electro-manipulation and separation of bio-particles using travelling electric field di-electrophoresis effects (Rizvi 2001). They also used a mask projection tool to successfully pattern three layers of a biosensor device. Laser mask projection method allows creation of a complete pattern in the metal layer so that complex fabrication process of printing micro-circuits can be done in a single pass (Rumbsy 1997). With the help of de-magnification ratio of the focusing lens, the laser's effectiveness can be controlled. The geometry and quality of the pattern can also be controlled by controlling the process parameters and focal position. In laser mask projection technique the complexity of the pattern is not considered an important factor as the quality of final pattern depends only on total area to be patterned and the energy required to remove the required amount of material (Rumbsy 1997). The processing time for a particular material depends upon beam spot size, spacing between holes, complexity of pattern(2D/3D), repetition rates, travel speed (X-Y) (Zheng 2001). 


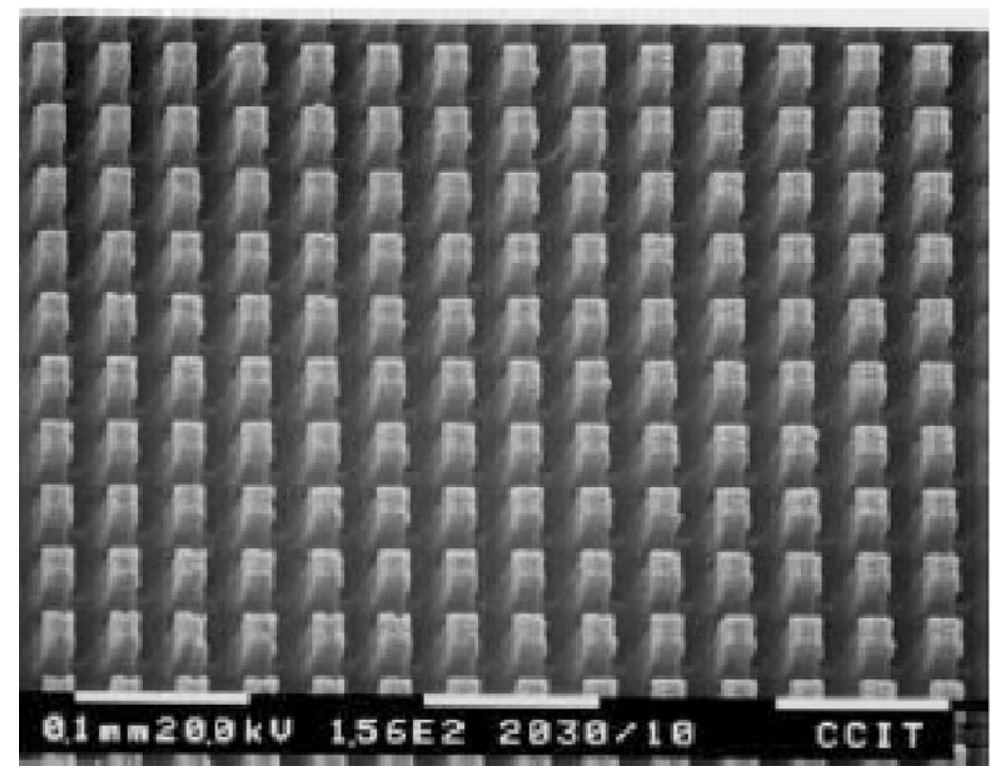

Figure 20 Glass probe array micro machined by laser ablation (Tseng 2004)

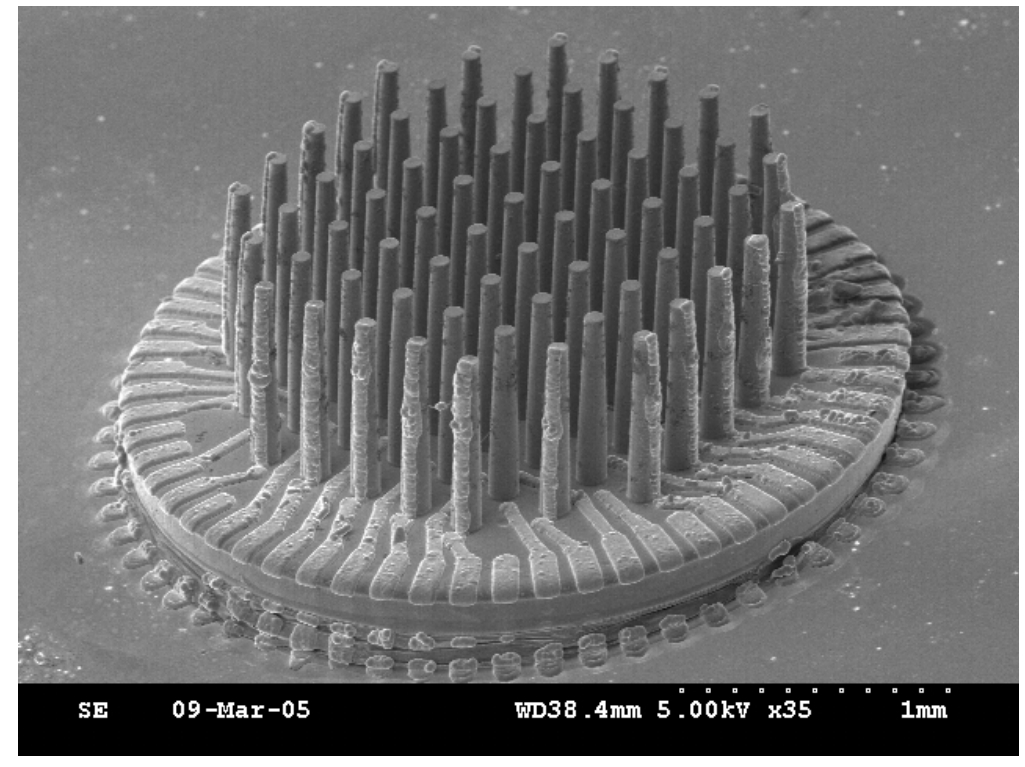

Figure 21 SEM image of 3-D Electrodes on a circular SU-8 substrate (Choi 2001) 

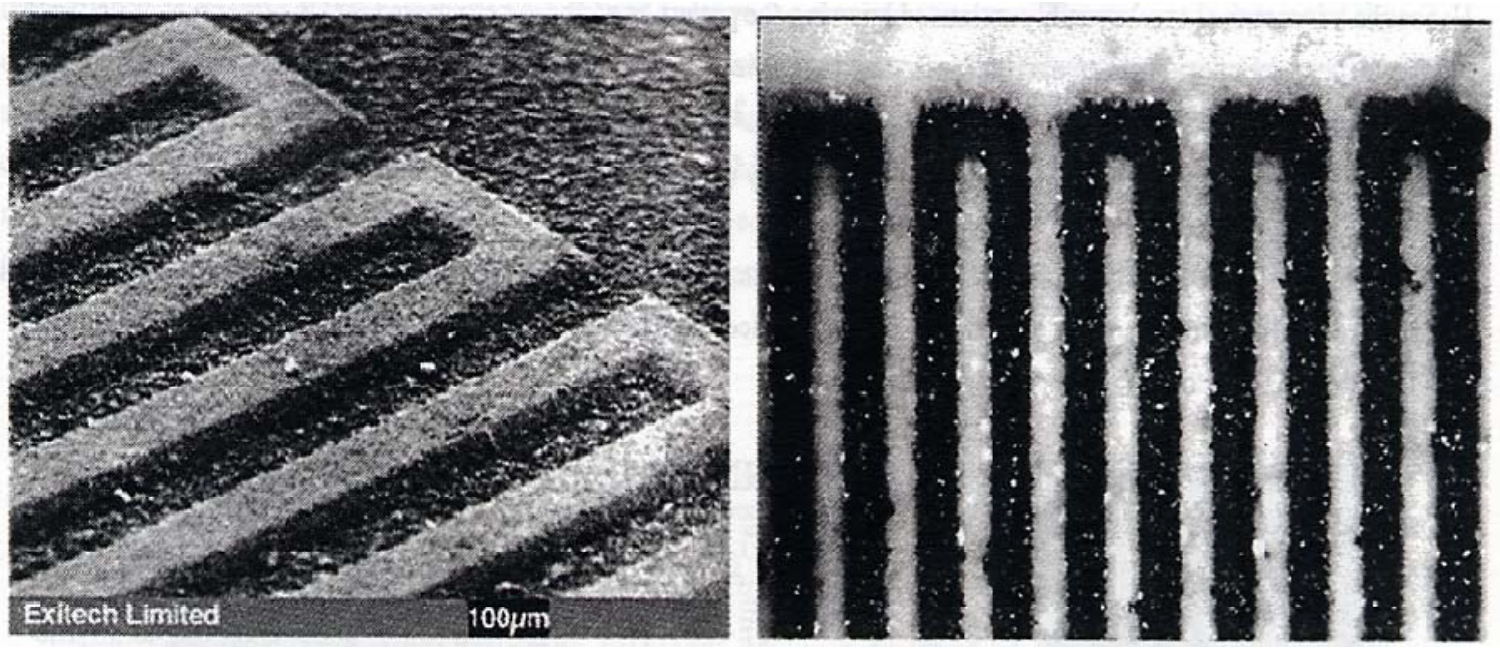

Figure 22 Printed thin film micro-circuit pattern by excimer laser (Rumbsy 1997)
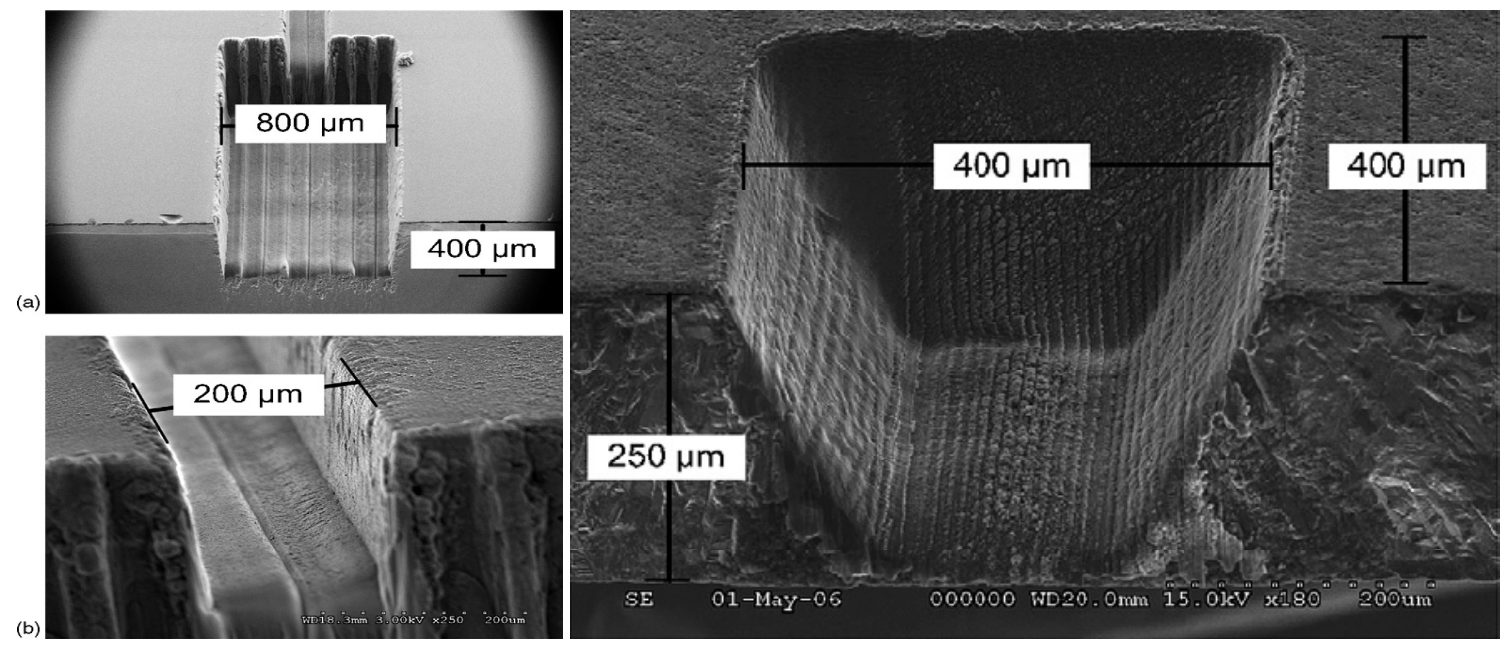

Figure 23 SEM images of laser milling on glass realized with optimal parameters: (a)

Rectangular cavity of $800 \mu \mathrm{m} \times 1200 \mu \mathrm{m} \times 400 \mu \mathrm{m}$, b) Close-up view of the surface finish ablated with the excimer laser and c) SEM image of a cavity milled in SiC (Desbiens 2007)

\subsubsection{Future Scope in MEMS}

The ablation phenomenon can be better analyzed by developing microscopic computer simulation models for better understanding of the quantitative information 
involved such as energy, temperature, pressure, atomic motions and qualitative conclusions (Zhigilei 1998). Thin film synthesis by pulsed laser deposition have many future applications in producing new and artificially structured materials and coatings (Lowndes 1996; Zhigilei 1998). To further enhance the application of excimer lasers, a better understanding of the nature of micromachining process if required through experiments and analytical models (Tseng 2004). Laser excitation in photoablation Vs. vibrational excitation remains a topic for research (Zhigilei 1998). For MEMS device fabrication, simplifying of the fabrication steps should be considered an important direction for development efforts (Choi 2001).

Photo-chemical etching and grafting of polymeric surfaces provide an interesting new method to get a good control of the nature of laser treated surface which is an important advantage in biomedical and biosensors research (Callewaert 2003). There is industrial demand for manufacture printed complex sensor devices at densities much higher than that can be achieved with conventional processes such as screen printing, etching or photo-tool processing (Rumbsy 1997). In MEMS device fabrication, it is important to produce vertical geometrical features with undercut walls, which recently has been paid less attention because it is impossible to predict the in detail shape of a three dimensional structure made using a given 2D mask pattern (Paterson 1999; William 2004). 


\section{CHAPTER V}

\section{PROBLEM STATEMENT}

In order to comprehend the transfer of laser energy into work material, an organized approach is employed for excimer laser micromachining of polymer samples with control over laser parameters. Excimer laser micromachining provides various benefits for polymer micromachining with its UV light output and high ablation rate due to short wavelengths. The experimental results from the investigation of polymer ablation would help in optimizing the process parameters for micromachining process for micro-patterns and MEMS device fabrication. Polyurethane (PU) polymers have received widespread interest for excimer laser micromachining due to their lower ablation threshold and high quality of micromachining. The main objective of this research is to understand the irradiation behavior of polyurethane polymer when exposed to different operating conditions during excimer laser micromachining.

The first objective of this investigation is to conduct laser micromachining using experiments with an excimer laser on polyurethane (PU) polymer under different 
operating conditions, such as variance in repetition rates, fluence $\left(\mathrm{mJ}, \mathrm{J} / \mathrm{cm}^{2}\right)$, number of pulses $(\mathrm{N})$, and mask size.

The second objective of this investigation is to compare the ablation mechanism and ablation rates in air and underwater environments. Furthermore, to analyze the ablation results for differences for both environments. The surface morphology and topography will be analyzed using an optical microscope, surface profiler, and MicroXAM laser interference microscope.

The third objective of this investigation is to use computer generated NC codes or G-codes to perform translational movements in the $\mathrm{X}-\mathrm{Y}-\mathrm{Z}$ axes to generate micropatterns for different applications, also to analyze the effect of mask diameter on pattern design and seam quality.

The fourth objective of this investigation is to optimize process parameters to obtain high quality micro-machined surfaces or patterns, such as micro-gears and microcircuits. 


\section{CHAPTER VI}

\section{EXCIMER LASER EXPERIMENTAL SETUP AND OPERATING PROCEDURE}

\subsection{Introduction}

Micromachining was carried out using a short-pulse medium-power Lambda Physik COMPex 205i excimer laser generation system. The laser generation system can deliver a laser beam with nominal pulse duration of $25 \mathrm{~ns}$ with a maximum of $650 \mathrm{~mJ}$ of energy at a maximum of $50 \mathrm{~Hz}$ repetition rate at $248 \mathrm{~nm}$ wavelength for krypton fluoride $(\mathrm{KrF})$. The system consists of five main components, namley, (1) Laser beam generation system, (2) Optical delivery system, (3) Three-axis motion controller system with a computer workstation, (4) Gas delivery and exhaust system, (5) Safety equipment and sensors. A computer workstation is used to interface the laser generator system with the PSO stage and motion controller connected through hardwired motherboard. The laser beam generation system can be triggered to output laser beam either internally via the computer workstation or by external keypad controller. 


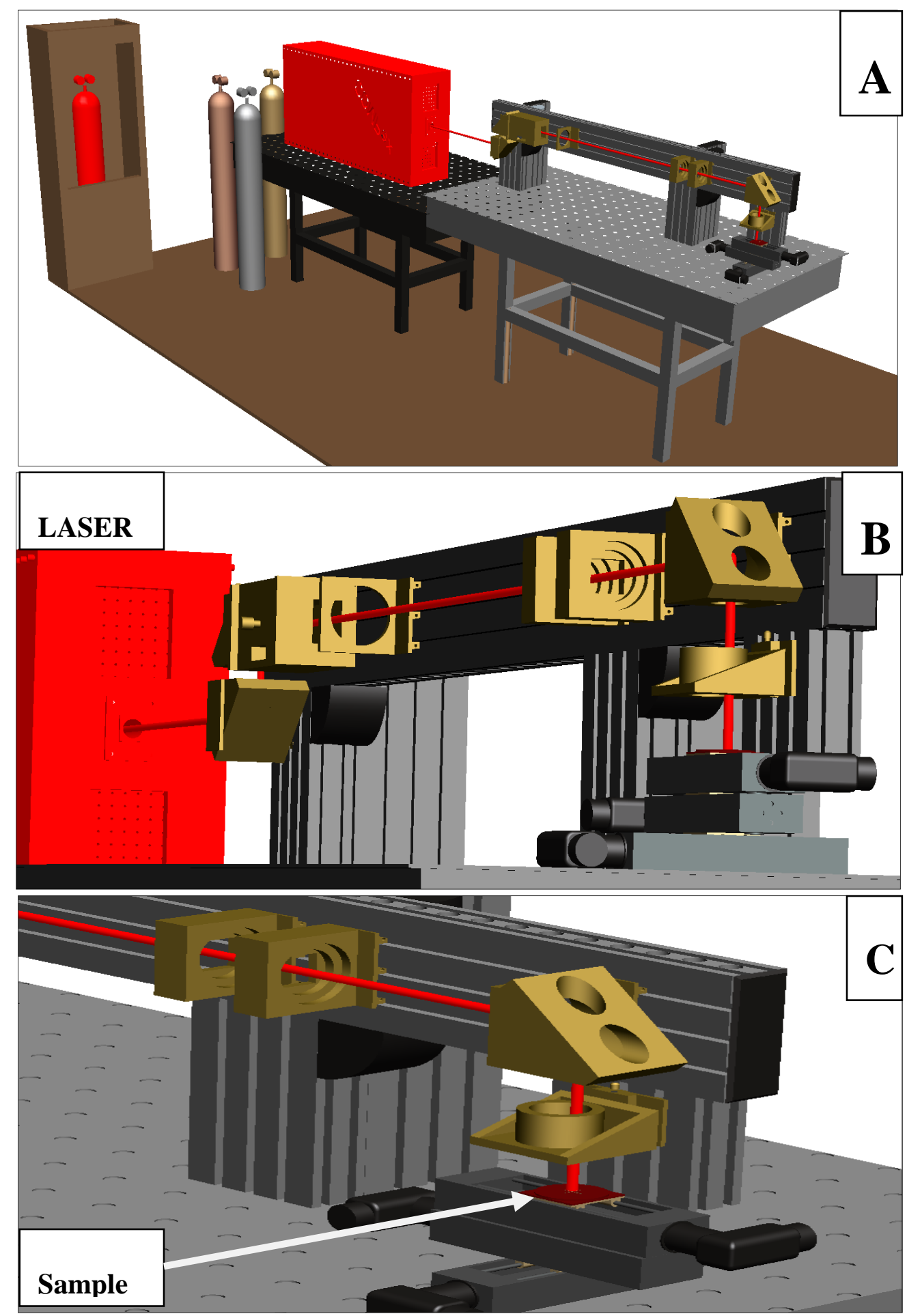

Figure 24 a) Schematic of the laser system, b) Optical delivery system, c)

Doublet and workpiece arrangement 


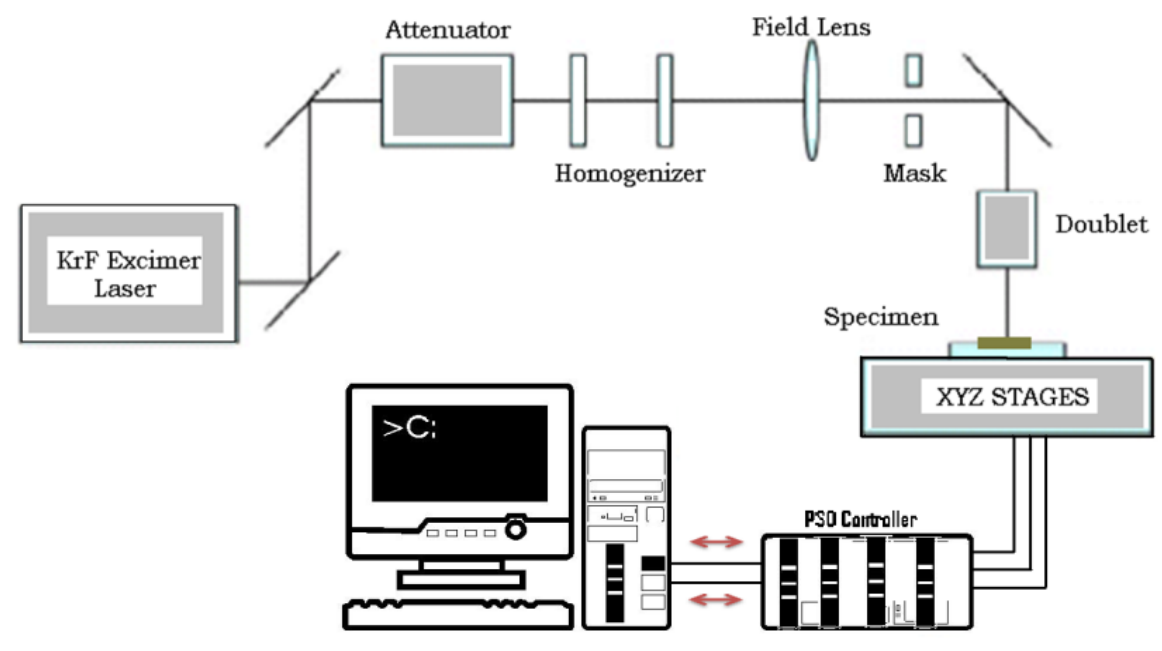

Figure 25 Schematic of the experimental setup for excimer laser micromachining (Gould 1959)

\subsection{Laser Beam Generation System}

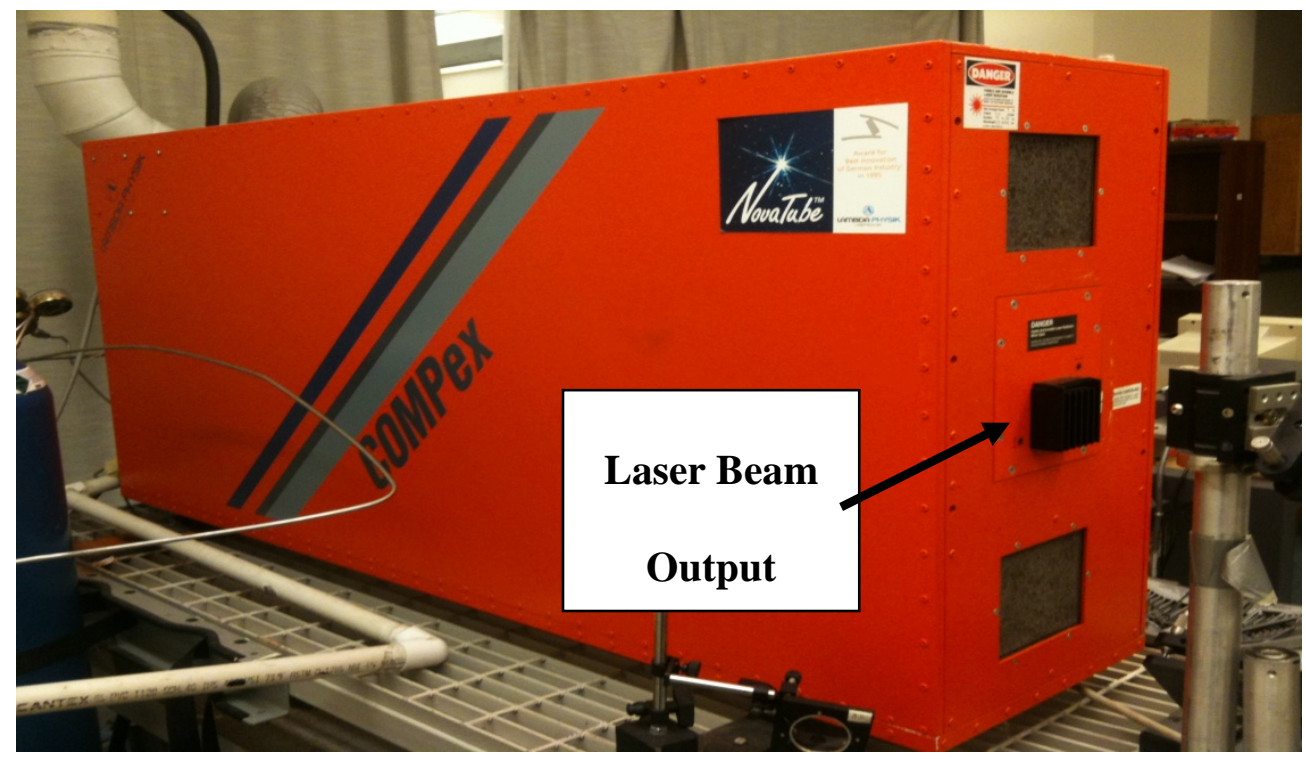

Figure 26 A KrF excimer laser generation system enclosure 
The laser generation system is a Lambda Physik COMPex 205i excimer laser connected to a $115 \mathrm{~V}$ at $25 \mathrm{~A}$ power supply which produce a high power, short pulse $(\mathrm{FWHM}=25 \mathrm{~nm})$ laser beam. It is installed in a dry and clean area where ambient temperature is maintained at $25^{\circ} \mathrm{C}$. The laser output is controlled using external or internal triggers using computer workstation or handheld controller. Following are Lambda Physik specification from laser the operational manual given:

\section{Table 7 Lambda Physik COMPex 205i Excimer laser specifications}

\begin{tabular}{|l|c|}
\hline Wavelength (nm) : & $248 \mathrm{~nm}(\mathrm{KrF})$ \\
\hline Maximum Output Energy : & $650 \mathrm{~mJ}$ \\
\hline Maximum average power : & $30 \mathrm{~W}$ \\
\hline Normal Operating Voltage: & $22 \mathrm{~V}$ \\
\hline Maximum Repetition Rate: & $50 \mathrm{~Hz}$ \\
\hline Nominal pulse duration : & $25 \mathrm{~ns}$ \\
\hline Translational Stage Axis & $\mathrm{X}, \mathrm{Y}$ and Z \\
\hline Type of the homogenizer : & Dual-Axis \\
\hline Orientation of the laser beam : & Horizontal \\
\hline $\begin{array}{l}\text { Gas Mixture } \\
\text { Used(Type=Purity) }\end{array}$ & $\begin{array}{c}\mathrm{Ne}=99.995 \%, \mathrm{Kr}=99.99 \%, \mathrm{Fl}= \\
5 \%+\mathrm{He}=95 \%, \mathrm{He}=99.995 \%\end{array}$ \\
\hline
\end{tabular}

\subsection{Optical Delivery System}

The optical delivery system consists of various beam altering mechanisms to control amplitude, focus, range, divergence and intensity. It consists of an attenuator, homogenizer, mask, field lens and a doublet to control delivery of the beam. The attenuator consists of a coated attenuating element with a counter compensator plate 
whose angle can be varied using a hand driven dial which allows the transmission of beam to be changed between $0 \%-95 \%$.

Homogenizer transforms segments of laser light from generation system into a homogenous laser beam. It consists of two arrays of lens which are cylindrical in shape along with condenser lenses. Field lens gathers the laser light coming from homogenizer and converges the laser beam and directs it towards the mask. Depending upon the mask shape and size, laser beam takes the form of a replica of the mask shape and is allowed to pass through the mask. Mask sizes and shapes can be changed based on the type of laser operation.

Doublet, which can be adjusted, consists of two lenses of $50 \mathrm{~mm}$ radius mounted with the curved sides of the reflective lenses which converges and de-magnifies the mask image of laser light onto the workpiece.

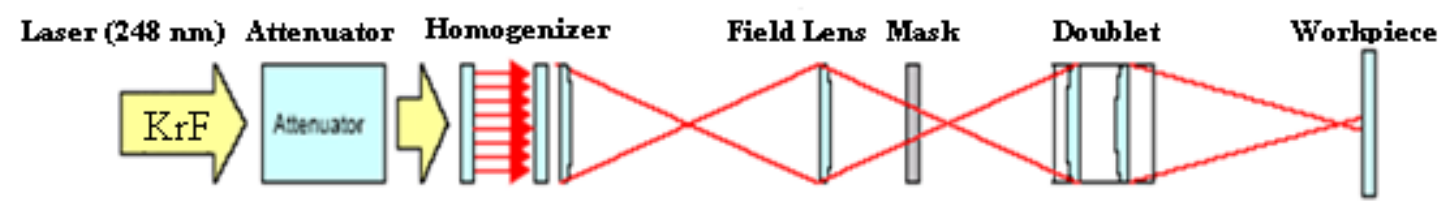

Figure 27 Schematic of the optical laser beam delivery system (Gould 1959) 


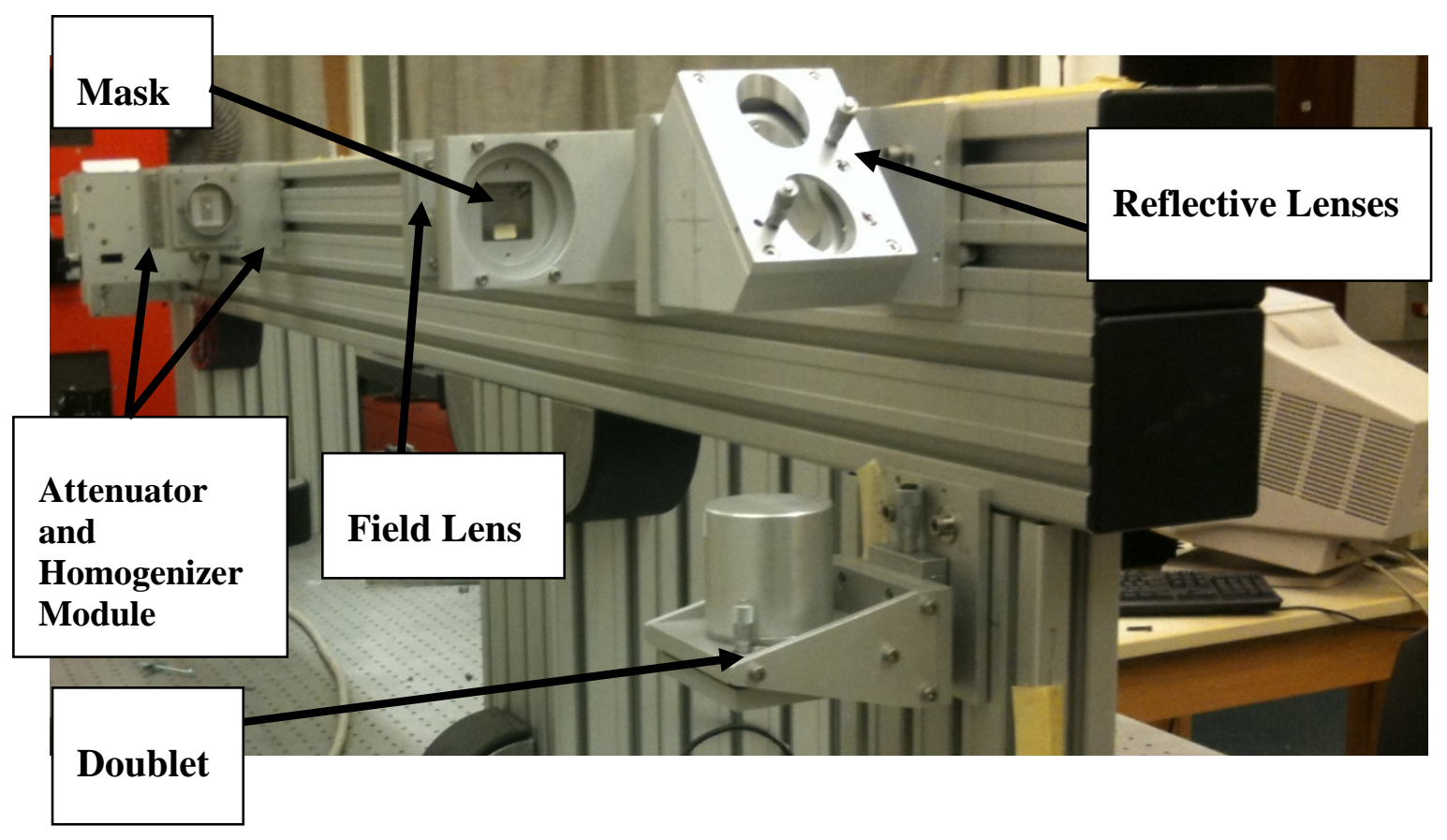

Figure 28 Excimer laser optical beam delivery system

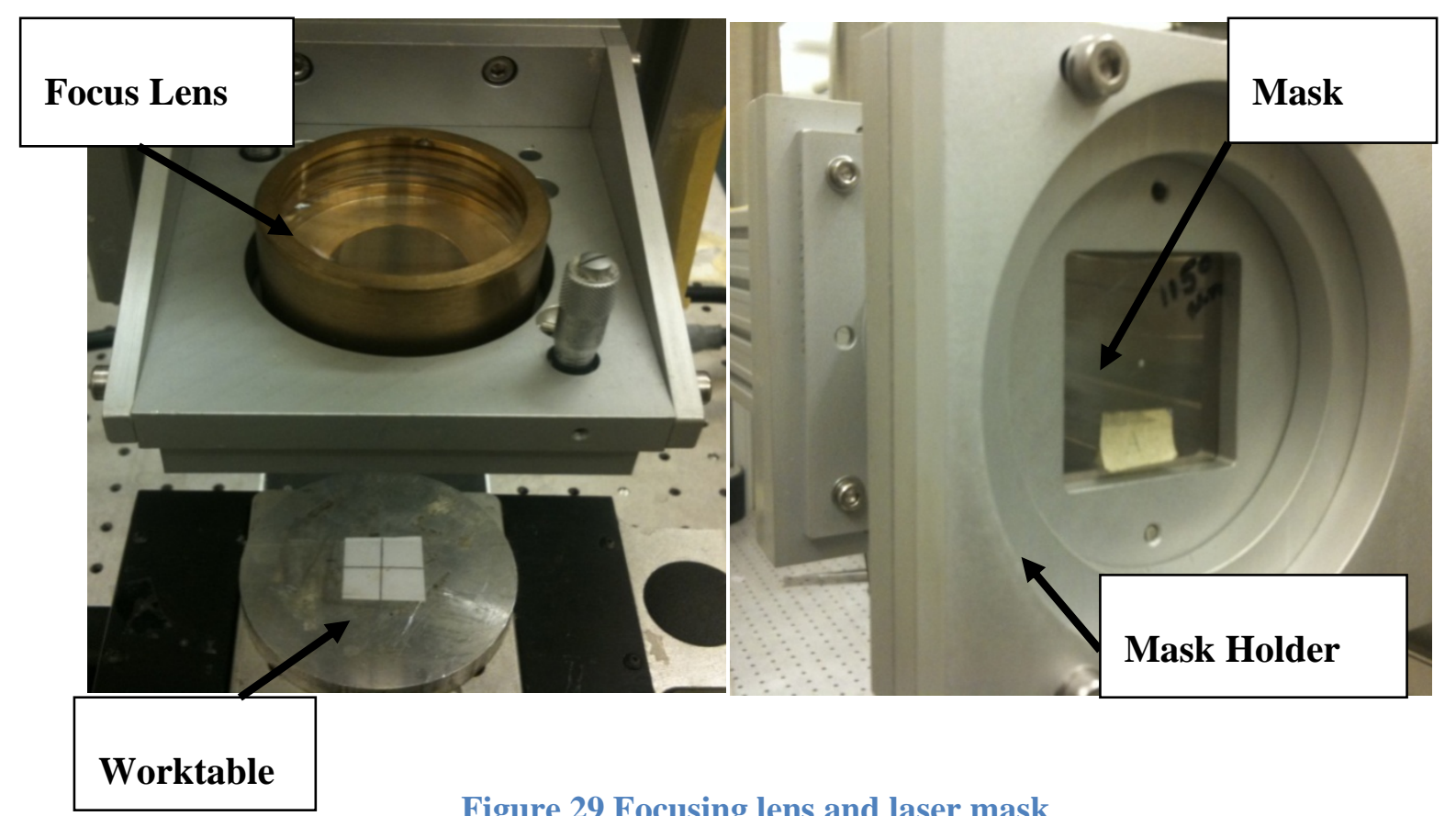

Figure 29 Focusing lens and laser mask 


\subsection{Stage and Motion Controller}

The stage and motion controller controlls the $\mathrm{X}, \mathrm{Y}$ and $\mathrm{Z}$ axis movements either manually or though computer workstation with the help of numerical codes or G-codes. It is made by Aerotech Co. and is used with three transitional stages as ATS100-150(XAixs), ATS100-100 (Y-Axis) and AVS105(Z-axis). The transitional stages are hardwired with the controller and the controller is interfaced with the computer workstation with a hardw are motion control card UNIDEX 500 with a PC-bus security key module attached to the printer port of CPU. The computer workstation is equipped with a MMI interface software along with CimCAD package which integrates the whole system to be a multifucntional, programmable and manually controlable motion control system.

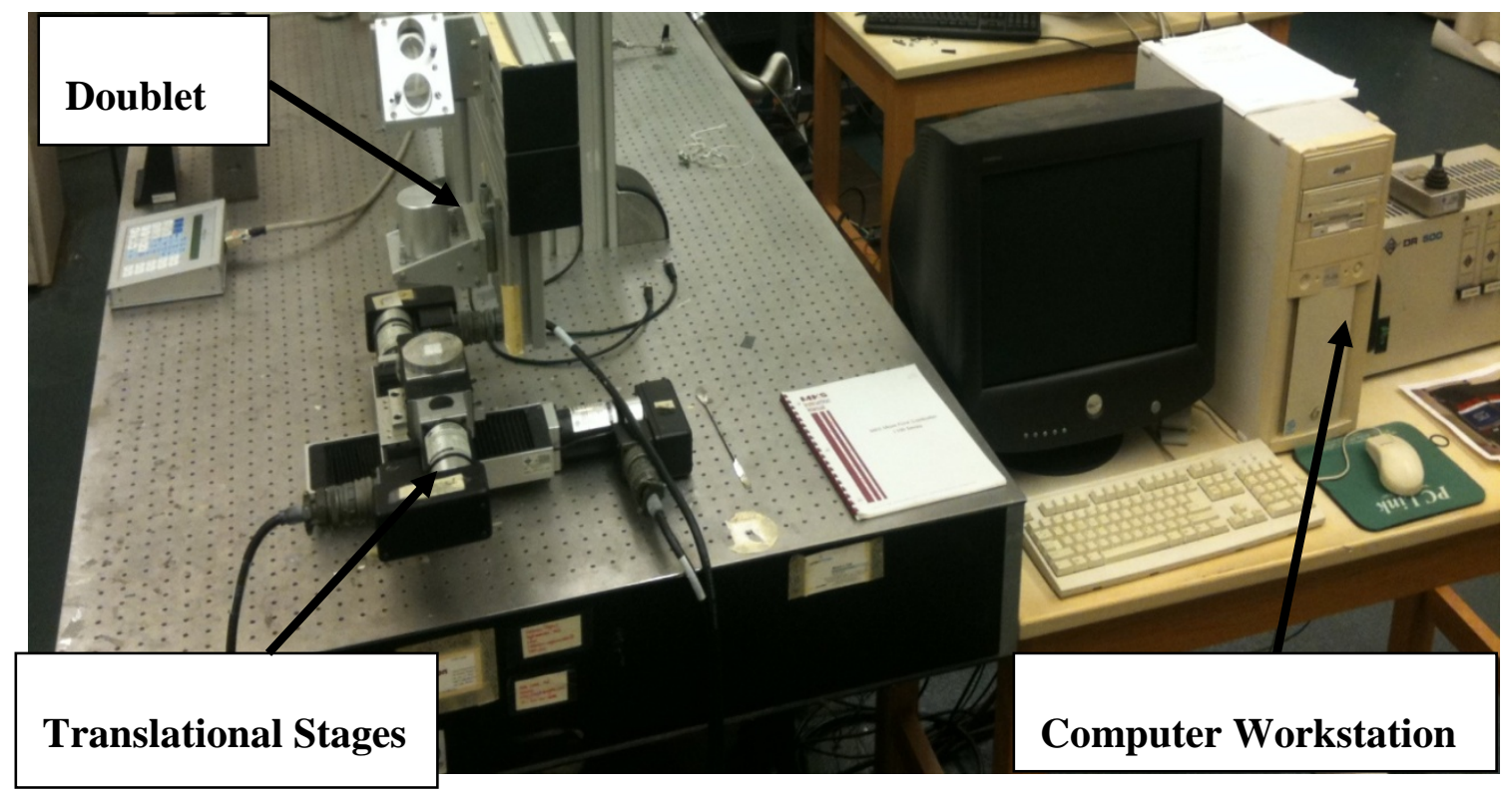

Figure 30 Computer workstation and translational (X,Y) stages 


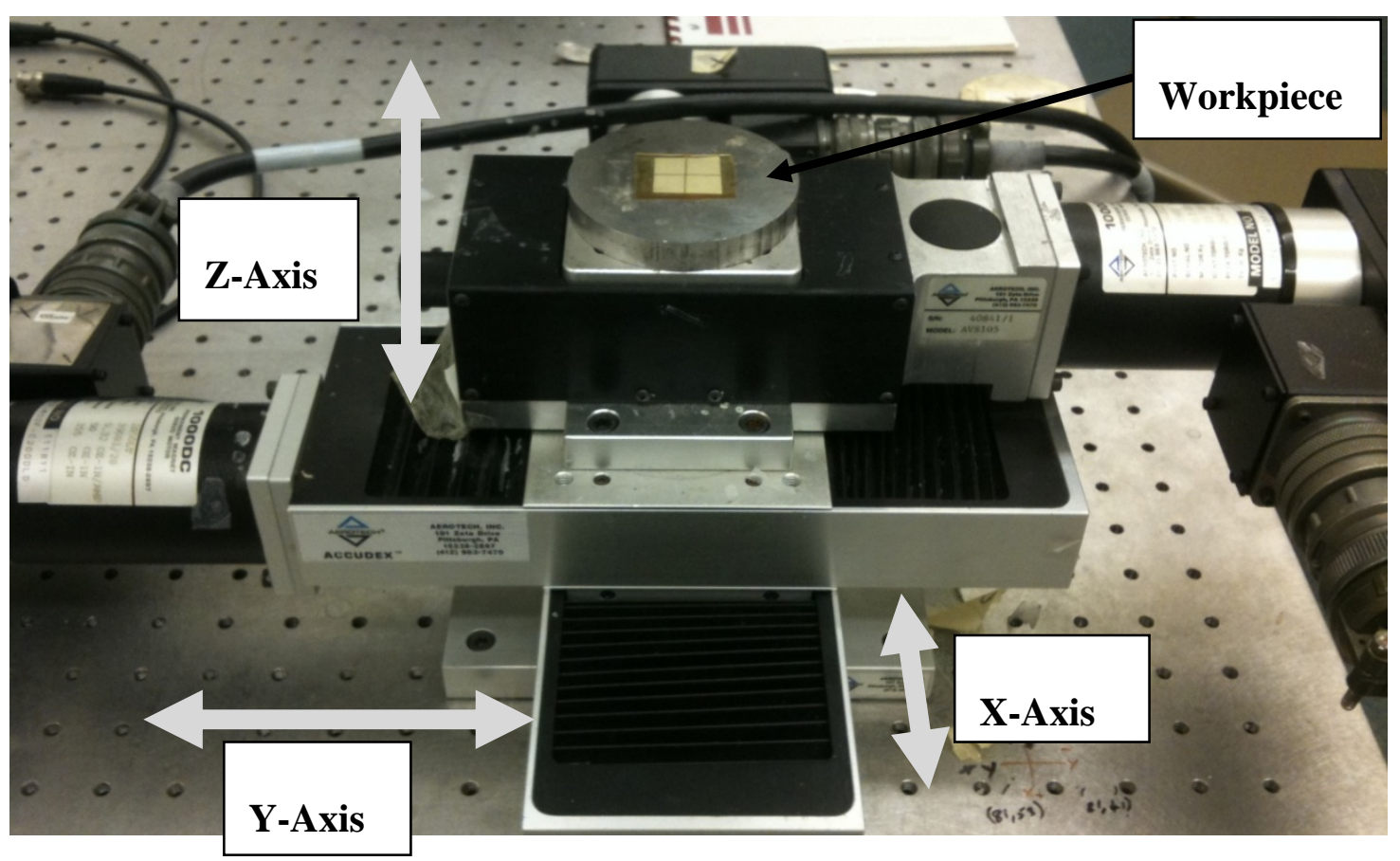

Figure 31 Translational stages and work sample

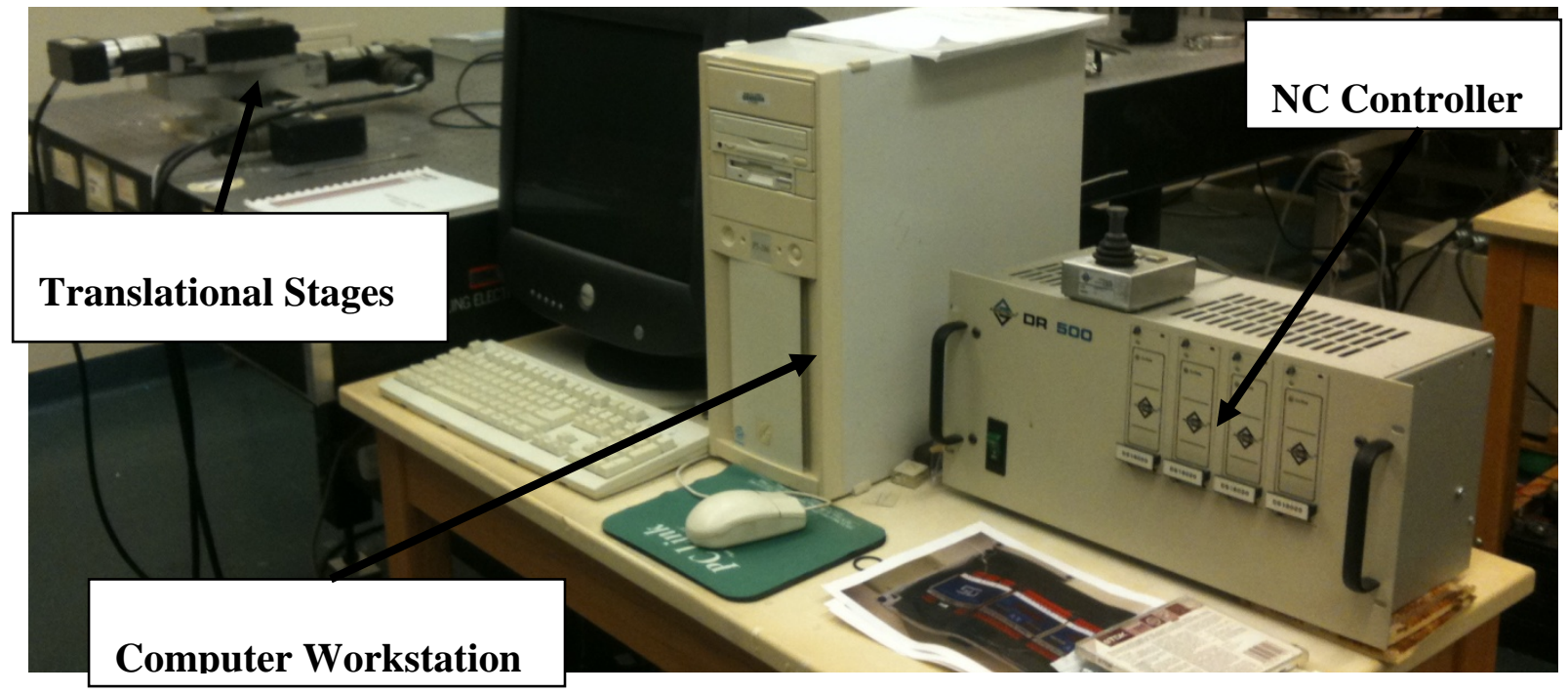

Figure 32 Stage and motion controller 

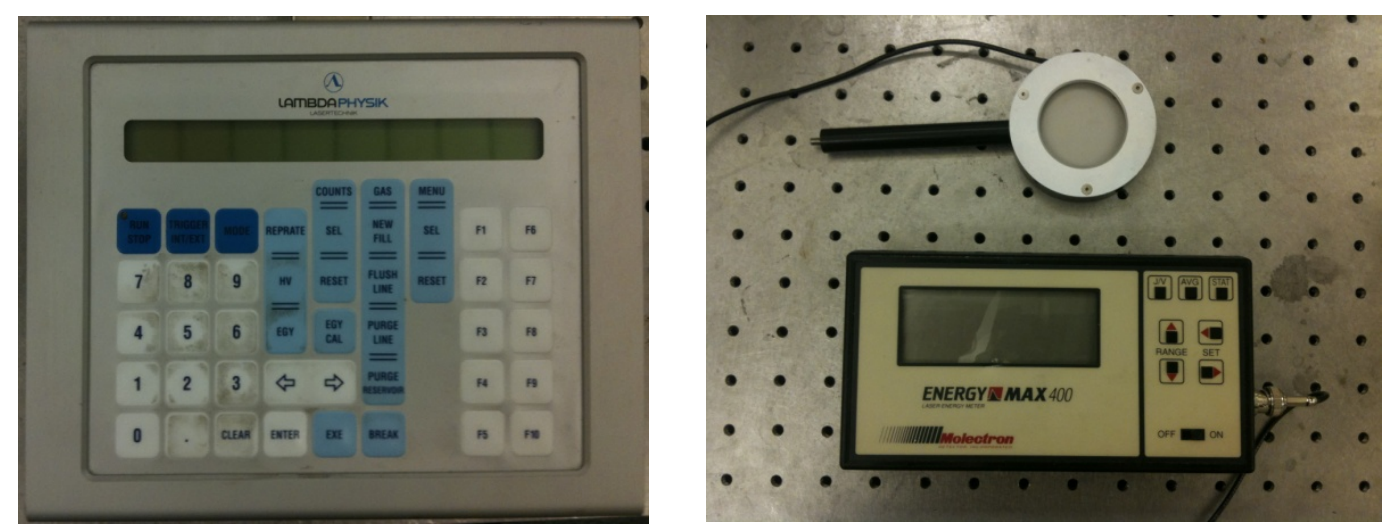

Figure 33 External control keypad and energy calibration device

\subsection{Gas Delivery and Exhaust System}

The gas mixture used in the system consists of four elements; 1) Buffer gas, 2) Rare gas, 3) Halogen gas and 4) Inert gas. Neon (Ne) is used as a buffer gas at $99.995 \%$ purity level, Krypton(Kr) is used as rare gas at $99.99 \%$ purity level, Halogen mixture consists of 5\% Fluorine $\left(\mathrm{F}_{2}\right)$ and $95 \% \operatorname{Helium}(\mathrm{He})$ and finally Helium is used as inert gas at $99.995 \%$ purity level. All gas cylinders are controlled using pressure regulators of 580 valve size and 3000psi pressure handling range.

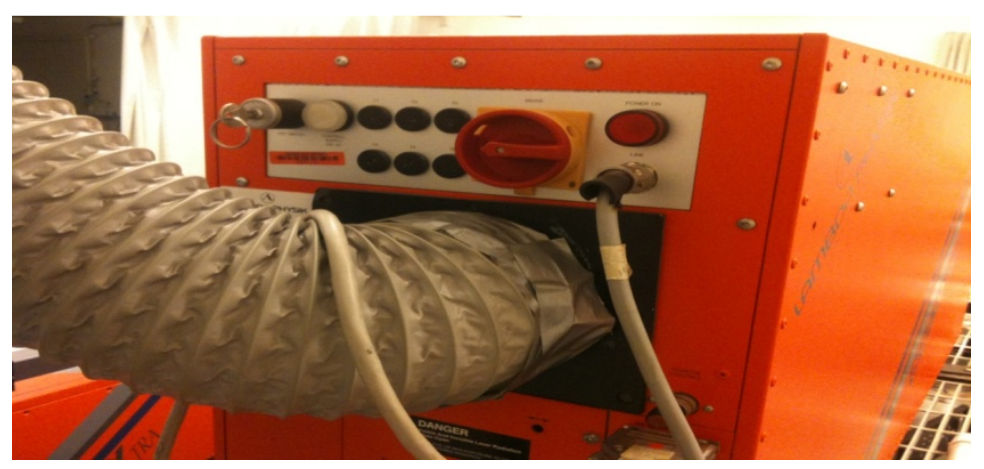

Figure 34 Laser module gas exhaust system 

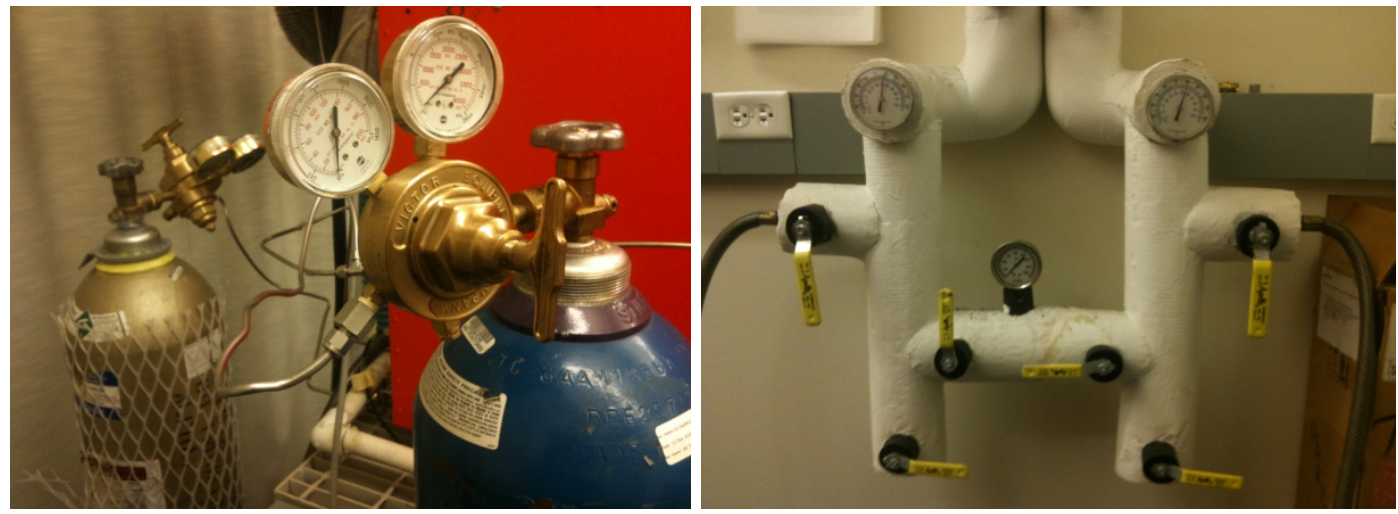

Figure 35 Gas cylinders and cooling water supply

\subsection{Safety Equipment and Sensors}

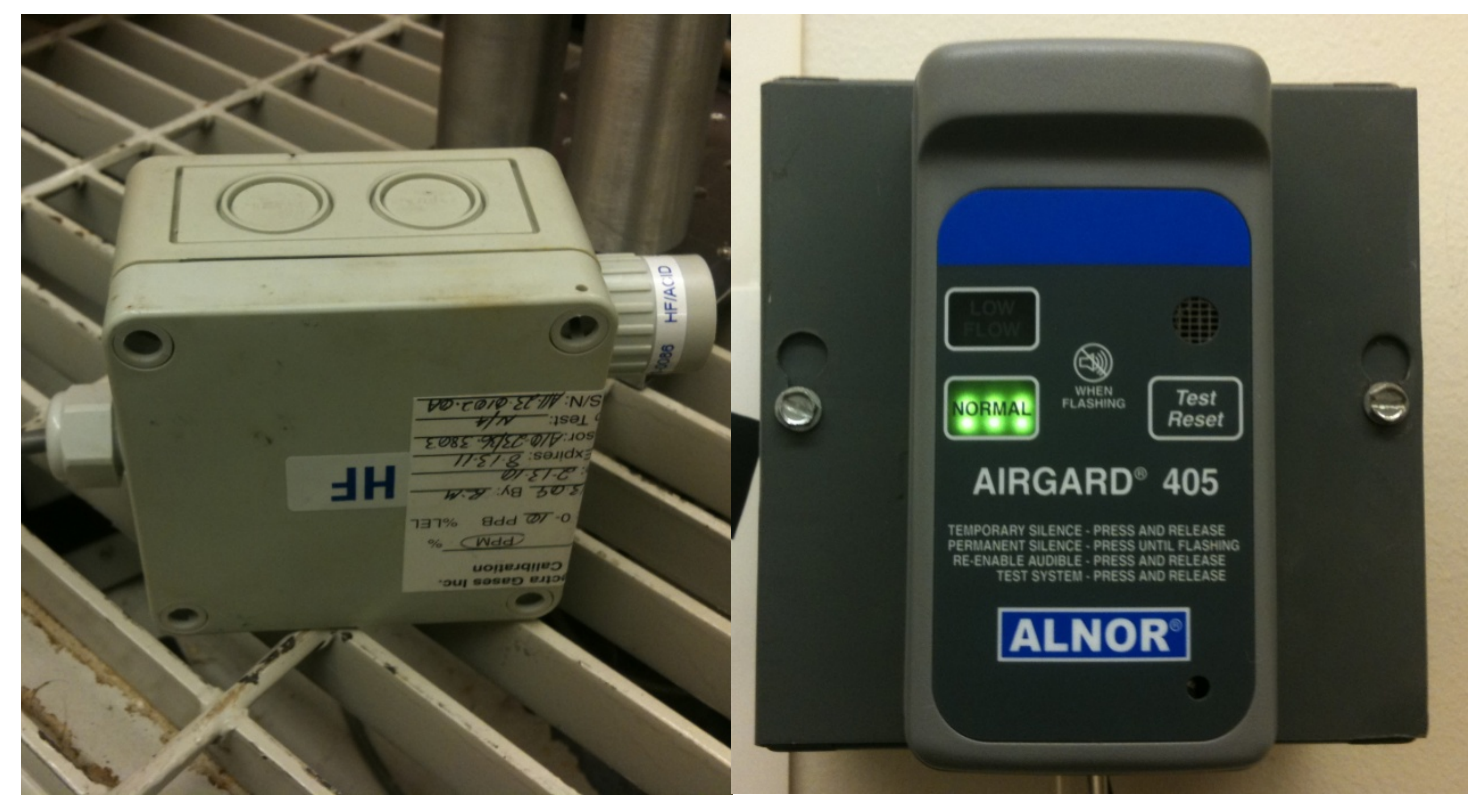

Figure 36 Safety sensor and hazard situation warning system

The safety sensors shown in Fig. 36 can detect any leaks in surrounding areas of laser for halogen mixture from its enclosure. Regular maintenance of safety sensors is carried out with weekly safety checks of sensors and alarm system. 


\section{CHAPTER VII}

\section{METHODOLOGY}

\subsection{Introduction}

The Excimer laser micromachining was carried out using the following steps: 1) Sample preparation, 2) Determination of laser fluence and focal point, 3) Calibration of input energy, 4) Laser micromachining under in air and underwater environments, 5) Ablation depth and Surface analysis using optical microscopy, and 6) Surface mapping using profilometer and MicroXAM laser interference microscope.

\subsection{Sample Preparation}

Following steps were taken in the preparation of the sample:

1. The sample has first cut from a large sheet to $10 \times 10 \mathrm{~mm}$ size and placed in a beaker with a detergent solution for cleaning purposes.

2. The sample was rinsed with distilled water.

3. The sample was again placed in a beaker containing distilled water and then subjected for further cleaning in an ultrasonic bath for 2 minutes.

4. Methanol was used to rinse the sample. 
5. The sample was placed in a beaker containing methanol and subjected to ultrasonic treatment for 2 minutes.

6. The sample was dried using compressed air.

\subsection{Determination of Laser Fluence, Average power and Focus Point}

The output energy per pulse from laser always decreases with its service time after a large number of pulses has been fired. It is considered a wise precautionary measure to ensure the proper output energy of laser beam using energy calibration equipment. Equation 7.1 is employed for calculation of laser fluence:

$$
f=\frac{E_{\text {avg }}}{\pi \cdot\left(\frac{D}{4}\right)^{2}}
$$

Where $\mathrm{E}_{\mathrm{avg}}$ is the average of pulse energy obtained following every 50 pulses, and D is the diameter of the mask, e.g. $1152 \mu \mathrm{m}, 555 \mu \mathrm{m}$ etc

$$
P_{\text {avg }}=E \times f
$$

Where $\mathrm{P}_{\text {avg }}$ is the average power output, $f$ is the repetition rates in $\mathrm{Hz}$, and $\mathrm{E}$ is the energy per pulse.

For laser focus point determination, the optical setup is used along with some preliminary tests and the best focus location and doublet settings were employed for rest of the experiments. 


\subsection{Calibration of Input Energy}

A Meletron M400 energy calibration meter and detector is used to measure the direct output laser energy at various spots on optical system. Different sets of energy calibration are performed with the help of operational manual of Meletron M400 energy meter.

\subsection{Laser Micromachining Under Different Environments}

An excimer laser with 25ns FWHM is used to machine polymer samples under different environments. A selective set of laser micromachining parameters are, given in Chapter 8, used to perform laser micromachining different samples.

\subsection{Optical Microscopy}

An optical microscope made by Nikon Model \#EPIHOT 290 is used to analyze the surface characteristics of the laser micromachined samples. The intensity of thermal damage, hole diameter, nature of ablation, and ablation depths are observed and compared. Following steps are used to analyze a sample:

1. Place the specimen (inverted) on stage

2. Center stage using $x-y$ controls at sides

3. Rotate objective lens turret so that $5 \mathrm{x}$ lens is beneath specimen

4. Turn on the microscope power

5. Adjust the light intensity; it should be less than half of the maximum available limit 
6. Adjust eyepiece lens, fine focus know as required to focus on a surface

7. Move X-Y stage controls to view different areas

8. Re-center stage then turn turret to use higher power objection lens, i.e. $5 \mathrm{x}, 10 \mathrm{x}$, 20x, 50x and 100x

9. Re-center or re-adjust fine focus using fine or coarse knobs

10. Use computer workstation to obtain optical micrographs, adjust various available parameters if necessary

11. Turn off microscope when finished

\subsubsection{Focus variation method}

Focus variation method is employed to determine the depth a micro-hole on a surface. At 20x magnification, the difference between the fine knob travel distant to properly focus at surface versus focus at bottom gives the proportional measure of hole's depth. Optical microscope's operational manual is used for determination of travel of eyepiece to shift the focus. Readings for all holes are recorded and used to develop depth results.

\subsection{Surface Mapping using Surface Profilometer}

A surface mapping profilometer made by NANOVEA Model \#PS50 is used to obtain surface maps of the samples. It uses a white light source and operates on an optical measurement principle called "Axial Chromatism" where light passes through an objective lens with a high degree of chromatic aberration. 
The surface profilometer is used to obtain $\mathrm{X}-\mathrm{Y}$ line profiles, ablation depth, ablation area for various holes and patterns. Following steps are employed to operate the surface profilometer:

1. Mount the optical pen $(130 \mu \mathrm{m}$ size $)$ for measurement

2. Wait for 5 minutes and record the dark signal (interference signal inherent to the sensor) and subtract from actual measurement.

3. Select the measurement mode; Topography or Thickness

4. Set the $\mathrm{Y}$-axis of the probe to make sure intensity bar remains between 5\%-99\%

5. Select the scanning parameters for frequency $(100 \mathrm{~Hz}, 300 \mathrm{~Hz}$, and $1000 \mathrm{~Hz})$, area $(\mathrm{mm})$, and step size $(5,10,30$ or $50 \mu \mathrm{m})$.

6. Start scan of the area

7. Observe the resulting scan and readjust, re-center, if necessary.

8. Save the data to a computer hard disk.

9. Open the data on a companion software (Professional 3D) for studying the results

10. Refer to operational manual for surface profiler or Professional 3D for further analysis of results.

\subsection{MicroXAM Laser Interference Microscopy}

A MicroXAM laser interference microscope is used for examining laser micromachined samples. It employs laser light to scan through the sample's surface using various wavelengths and ultimately provides a 3D scan, X-Y line profiles etc. 


\section{CHAPTER VIII}

\section{EXCIMER LASER MICROMACHINING OF POLYURETHANE}

\subsection{Introduction}

Polyurethane (PU) is a thermoplastic polymer which shows melting at ablation due a variety of primary and secondary structures (Suzuki 1998). As a result of excimer laser excitation during ablation the process many phases are observed such as temperature elevation, thermo-chemical decomposition, surface fragmentation and ejection of debris (Mackay 1995; Callewaert 2003; Kruusing 2004). It is highly desired that the material removal rates are related to the etch depth data for understanding of the ablation dynamics leading to a particular etched surface profile. Using excimer laser, inducing a significant chemical change on the surface of polyurethane impacts a number of technologies, such as tissue engineering, MEMS devices, and semiconductor manufacturing. At present, polyurethane (PU) is considered to be a best compromise material available, in terms of biocompatibility, mechanical flexibility, and strength. PU is practically very important and widely used as industrial and commercial products, i.e. MEMS devices, heart valves. 


\subsection{Ablation Characteristics of Polyurethane}

\subsubsection{Experimental Procedure}

A polyurethane $(\mathrm{PU})$ sheet $(1 \mathrm{~mm})$ is used as work material after cleaning its surface with a chemical solution to ensure a debris free surface. Laser mask sizes of 180 $\mu \mathrm{m}, 300 \mu \mathrm{m}, 455 \mu \mathrm{m}, 555 \mu \mathrm{m}$ and $1152 \mu \mathrm{m}$ are employed for laser beam delivery. The surface of the polymer is then exposed to laser energy for 5 seconds from $150 \mathrm{~mJ}$ to $500 \mathrm{~mJ}$ and with repetition rates from $1 \mathrm{~Hz}$ to $50 \mathrm{~Hz}$. The reason for this is to observe range of ablation depths at micro level with two goals; a) Change in ablation depth with respect to change in input energy at constant repetition rates, b) Change in ablation depth with respect to change in repetition (number of pulses) rate at constant pulse input energy. The computer workstation connected to the translational stages was utilized by PSO/G codes for precise translational movements or workpiece under incident laser beam. The computer aided design (CAD) was created using CimCAD ${ }^{\mathrm{TM}}$ and PSO-G codes are generated for the translational stages to externally trigger the laser. A scaling factor routine is carried out on G-codes to allow smaller gear pattern diameters to be etched from an original design. After a series of experiments, the laser micromachining process is repeated for different environments as under air and underwater to observe the differences in ablation dynamics of polymer. For underwater experiments $20 \mathrm{ml}$ of water is poured into a water container having the polymer sample completely submerged. The water container is shown in Fig. 37 as below: 


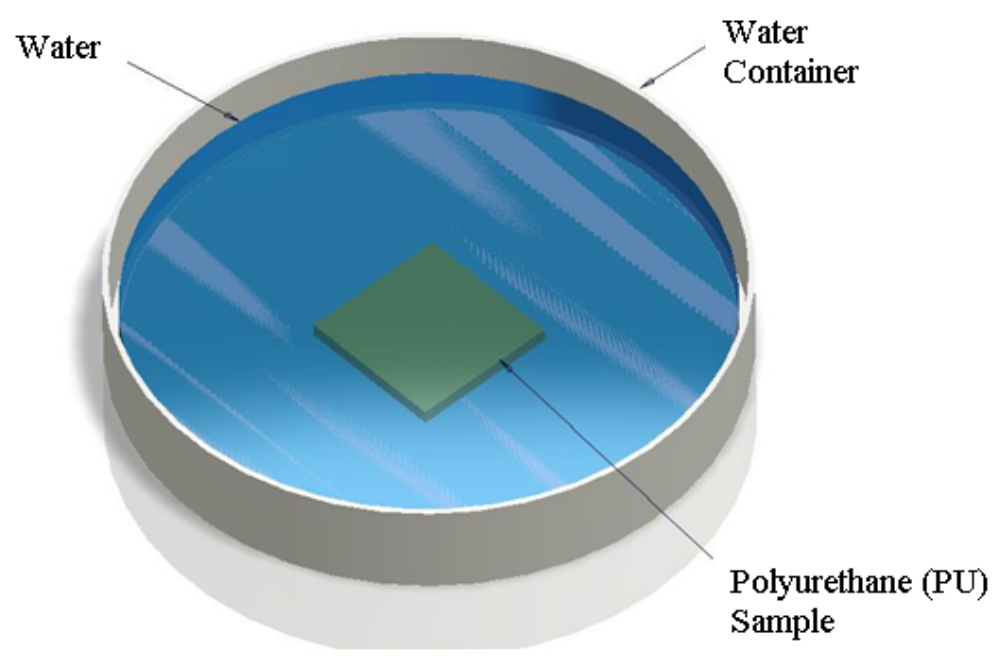

Figure 37 Schematic of the setup for micromachining underwater

The polymer sample is properly cleaned and then subjected to laser energy followed by examination under optical microscope, surface profilometer and MicroXAM surface mapper. Results of micromachining under air and underwater are then compared. Surface mapping data is used for understanding of ablation dynamics and developing hypothesis. Table 8 list the important properties of the polyurethane (PU) sample used. 
Table 8 Properties of polyurethane (PU) polymer (NICNAS 2000)

\begin{tabular}{|c|c|}
\hline Hardness & $60-88$ \\
\hline Tear Strength & $100-500 \mathrm{Ibs} . /$ inch \\
\hline Operating Temp. & $-65^{\circ}$ to $+225^{\circ} \mathrm{F}$ \\
\hline Density & $1200 \mathrm{~kg} / \mathrm{m} 3$ \\
\hline Elastic Modulus & $69-690 \mathrm{MPa}$ \\
\hline Tensile Strength & $1-69 \mathrm{MPa}$ \\
\hline $\begin{array}{c}\text { Compressive } \\
\text { Strength }\end{array}$ & $138 \mathrm{MPa}$ \\
\hline Flexural Strength & $5-31 \mathrm{MPa}$ \\
\hline $\begin{array}{c}\text { Coeff. of Thermal } \\
\text { Expansion }\end{array}$ & $100-200\left(10-6 /{ }^{\circ} \mathrm{C}\right)$ \\
\hline $\begin{array}{c}\text { Thermal } \\
\text { Conductivity }\end{array}$ & $0.209\left(\mathrm{~W} / \mathrm{m}-{ }^{\circ} \mathrm{C}\right)$ \\
\hline Specific Gravity & $1.03-1.5$ \\
\hline $\begin{array}{c}\text { Water Absorption }(\% \\
\text { weight increase) }\end{array}$ & $0.2-1.5$ \\
\hline Dielectric Strength & $300-500(\mathrm{~V} / \mathrm{mil})$ \\
\hline Polymer Class & Thermoset \\
\hline $\begin{array}{c}\text { Processing } \\
\text { Temperature }\end{array}$ & $6-121\left({ }^{\circ} \mathrm{C}\right)$ \\
\hline Molding Pressure & $1-31 \mathrm{MPa}$ \\
\hline $\begin{array}{c}\text { Base } \\
\text { Ester }\end{array}$ \\
\hline
\end{tabular}

\subsubsection{Results and Discussion}

Fig. 38 shows a preliminary test of ablation for polyurethane samples at a constant pulse enegy of $200 \mathrm{~mJ}$ with variable repetition rates $(1 \mathrm{~Hz}$ to $50 \mathrm{~Hz})$ under air environment. Significant ablation depth is observed from $5 \mathrm{~Hz}$ (25pulses) at $10 \mu \mathrm{m}$ with an average increase (etch rate) of $2 \mu \mathrm{m}$ per pulse up to $15 \mathrm{~Hz}$ (75pulses). Between $15 \mathrm{~Hz}$ to $30 \mathrm{~Hz}$, an average increase of $3.5 \mu \mathrm{m}$ per pulse is observed. During $30 \mathrm{~Hz}$ to $50 \mathrm{~Hz}$, the ablation depth is increased at an average of $4.5 \mu \mathrm{m}$ per pulse. Fig. 38 shows a comparison of similar experimental tests. Ablation depths for different repetition rates are 
measured using focus variation method which yields an average etch depth of $\sim 0.2$ $\mu \mathrm{m} /$ pulse. Linear increase of ablation depth is observed over a range of repetition rates.
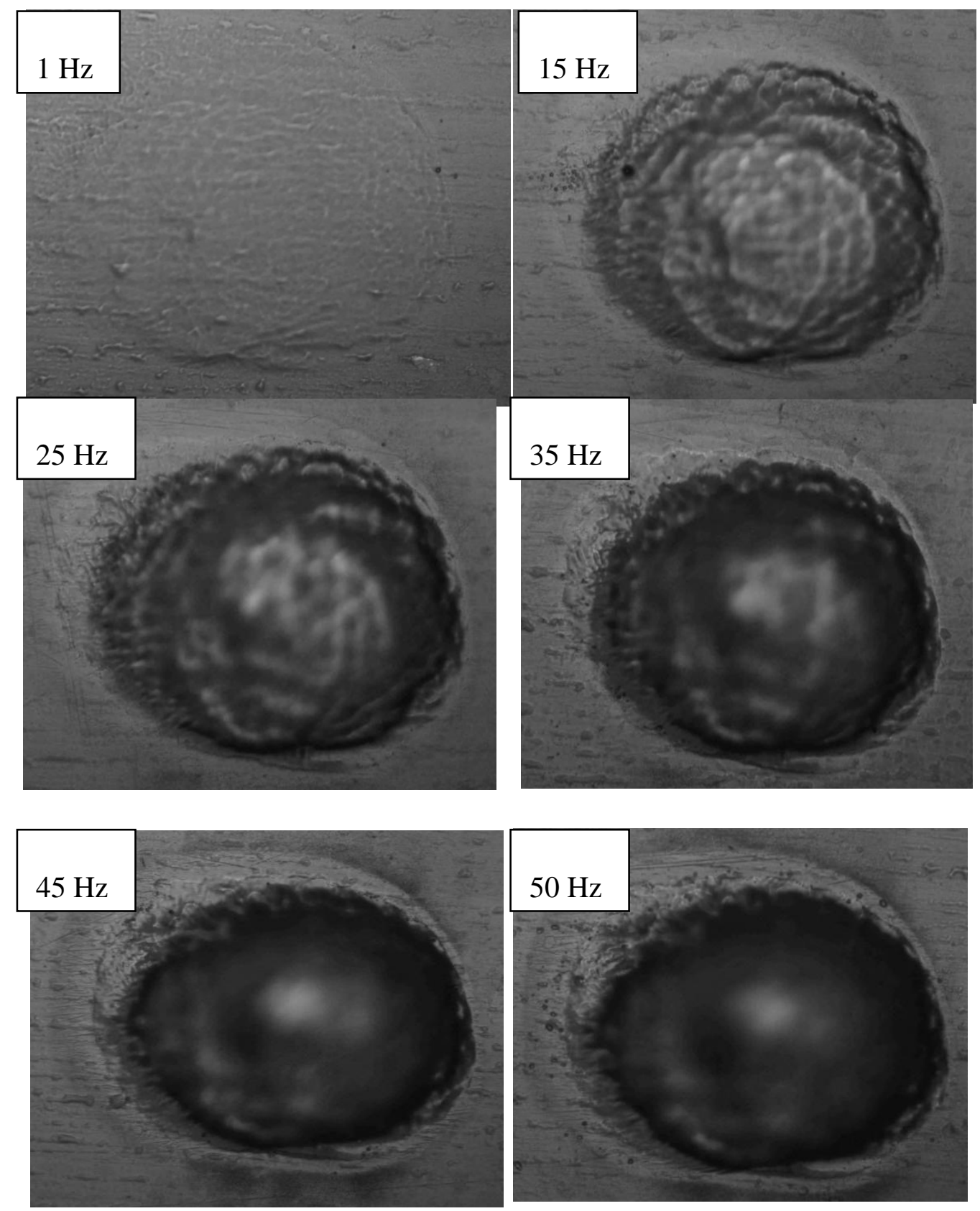

Figure 38 Optical micrographs of the top views of polyurethane samples ablated at pulse energy of $200 \mathrm{~mJ} / \mathrm{cm}^{2}$ at variable repetition rates ( $1 \mathrm{~Hz}$ to $50 \mathrm{~Hz}$ or 5 to 250 pulses) 


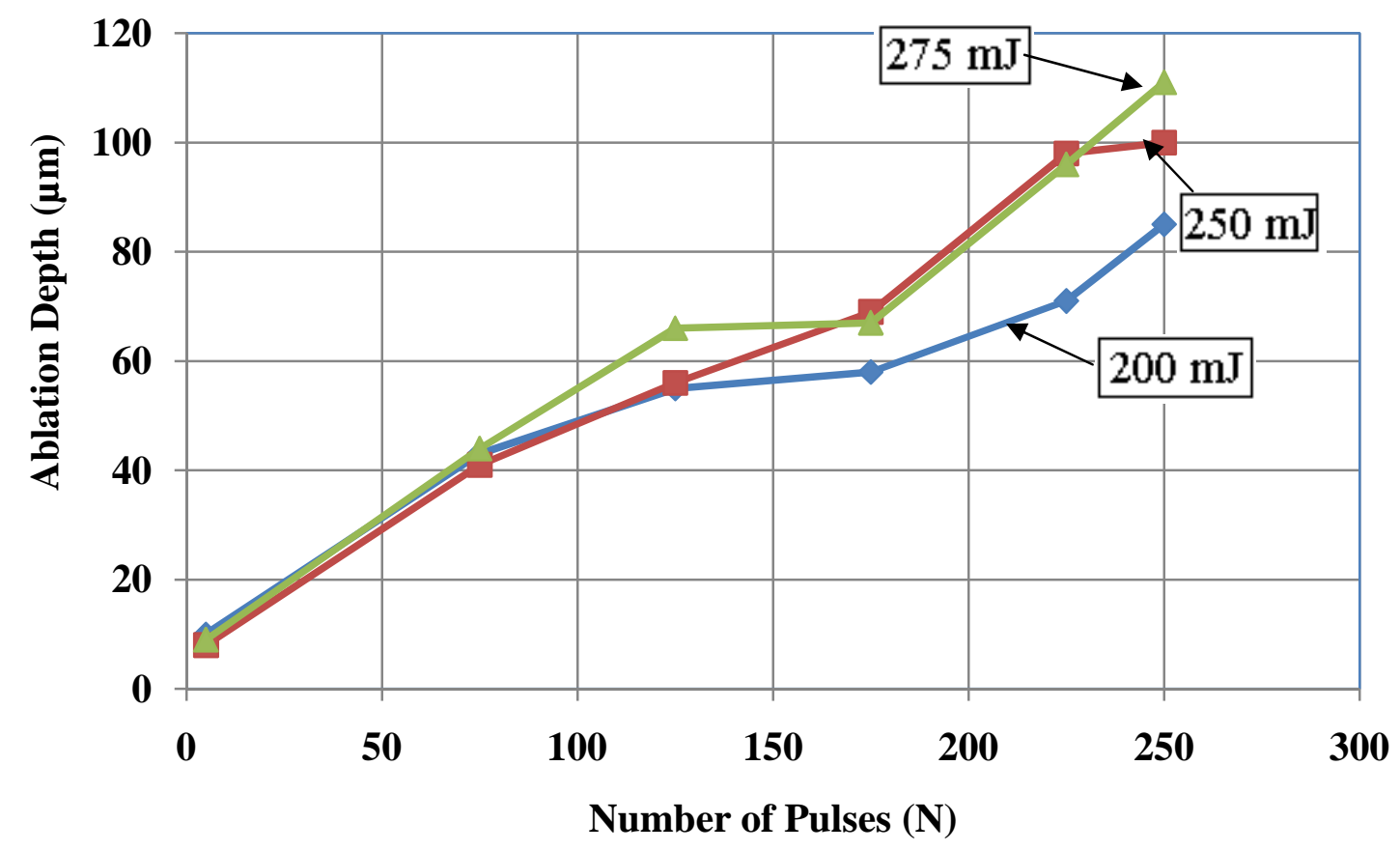

Figure 39 Variation of ablation depth with increase in number of pulses at pulse energies of $200 \mathrm{~mJ}, 250 \mathrm{~mJ}$, and $275 \mathrm{~mJ}$.

For $250 \mathrm{~mJ} \& 275 \mathrm{~mJ}$ pulse energy, the ablation depth increases linearly up to 15 $\mathrm{Hz}$ which imply rapid material removal; during $15 \mathrm{~Hz}$ to $35 \mathrm{~Hz}$ the change in ablation depth appears constant which could be due to plasma shielding or ejection of material. During $35 \mathrm{~Hz}$ to $50 \mathrm{~Hz}$, the ablation depth increases rapidly in both tests. Diameter of ablated hole is observed at $290 \mu \mathrm{m}$. At $50 \mathrm{~Hz}$, the diameter of the ablated hole increases to $345 \mu \mathrm{m}$ implying further ablation of the edges near HAZ due to high repetition rates of laser pulses. Minor increase in diameter of the hole geometry is observed as the number of pulses are increased. 


\subsubsection{Change in Ablation Depth}

Based on the previous preliminary experiments, a set of input process parameters are selected in combination with input energy and repetition rates for both air and underwater environments to investigate the most suitable operating range of parameters for polyurethane polymer. It is important to note here that all the ablated holes are observed to be non-through holes since it would require longer exposure time to have a through hole with limitations of maximum input energy. An attempt was made to obtain a through-hole but without success as longer exposure times for polyurethane cause excessive heat affected zones and very poor edge geometry. Following optical micrographs (Figs. 40 to 43) show ablation characteristics in air and underwater micromachining on polyurethane samples: 
A) In Air:

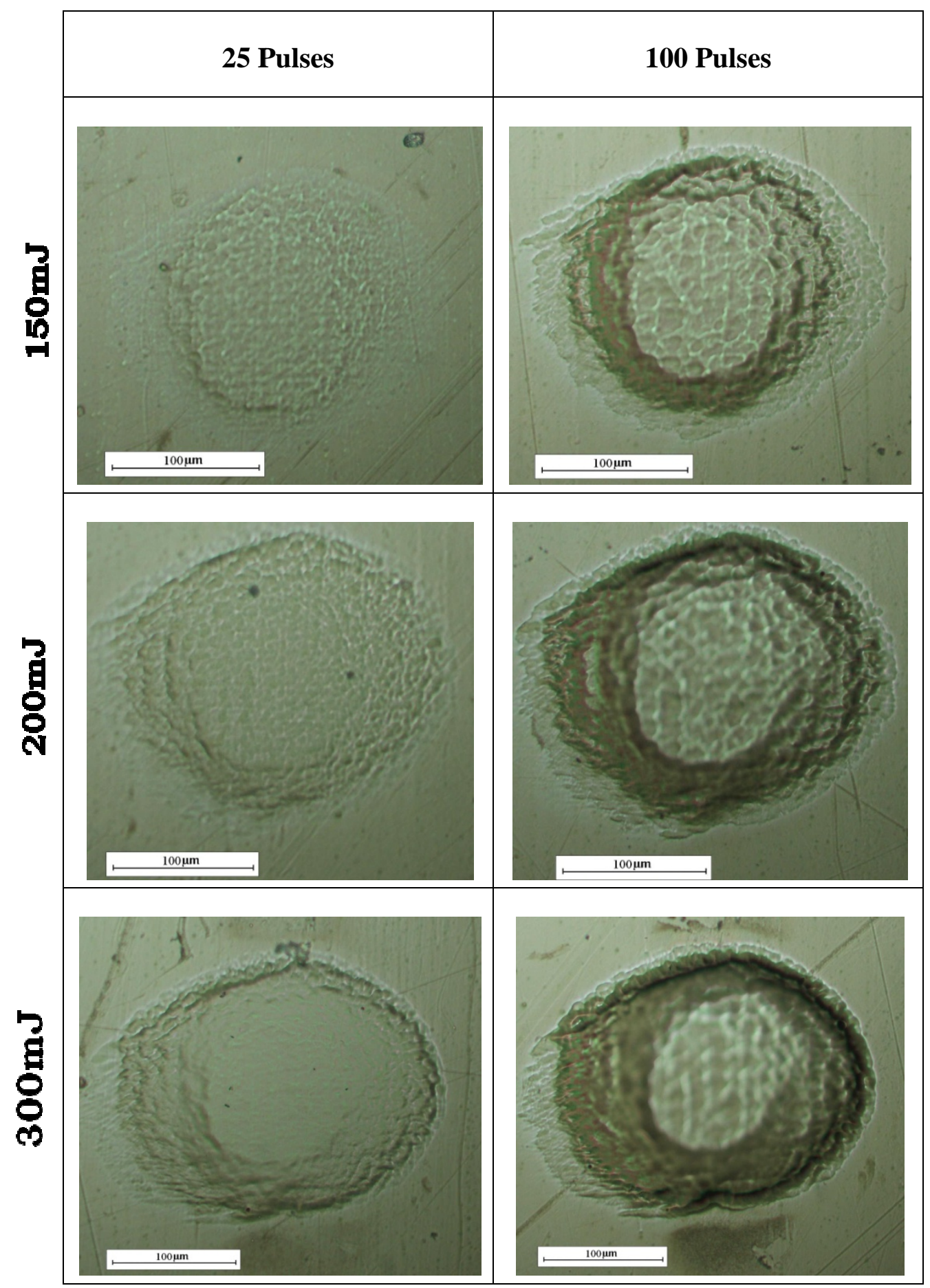

Figure 40 For in air environment, at 25-to-100 Pulses $(5$ to $20 \mathrm{~Hz}$ ) optical micrographs (at 20x) showing ablation 


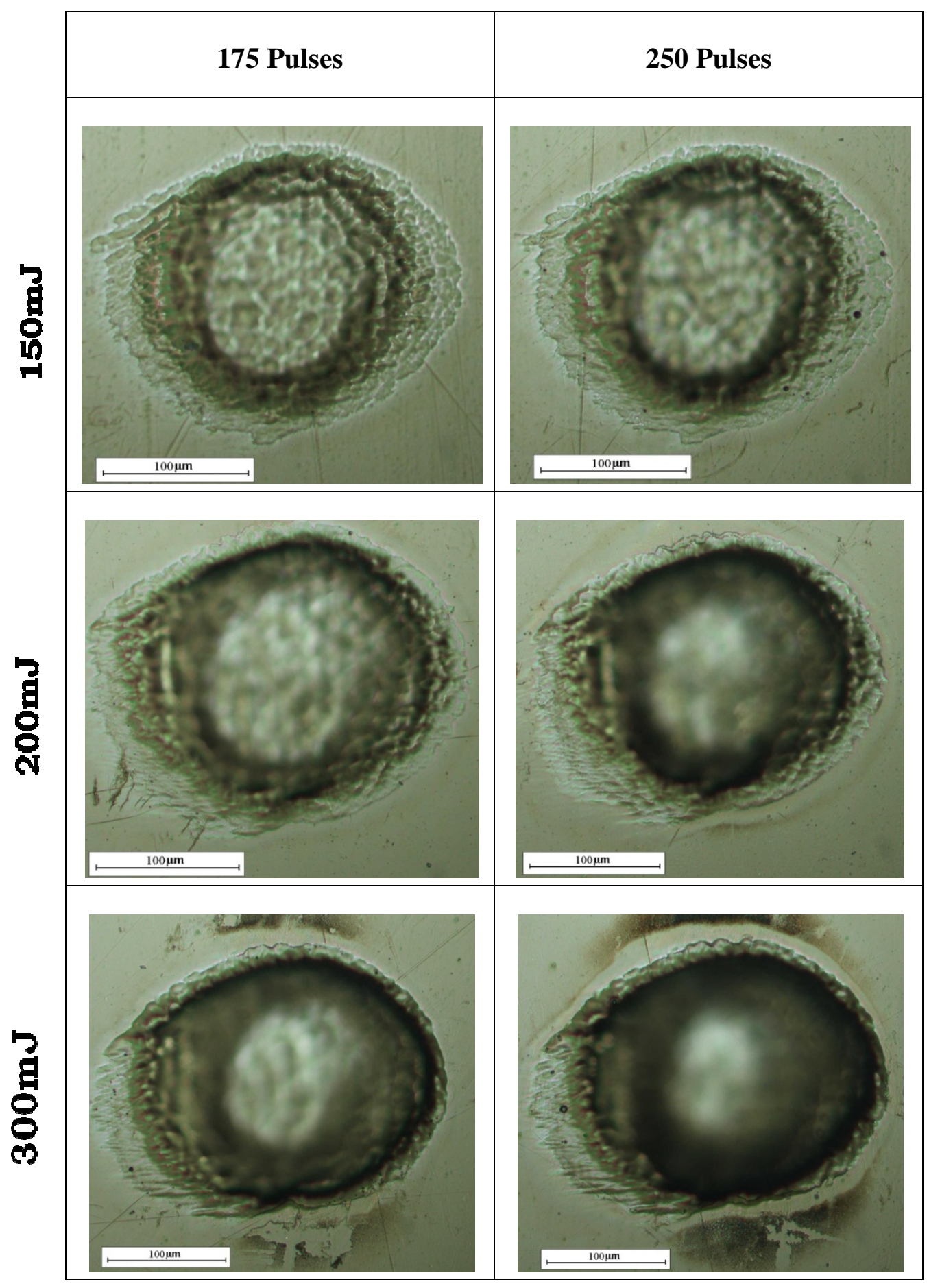

Figure 41 For air environment, at 175 to 250 Pulses (35 to $50 \mathrm{~Hz}$ ) optical micrographs (at 20x) showing ablation 
B) Underwater

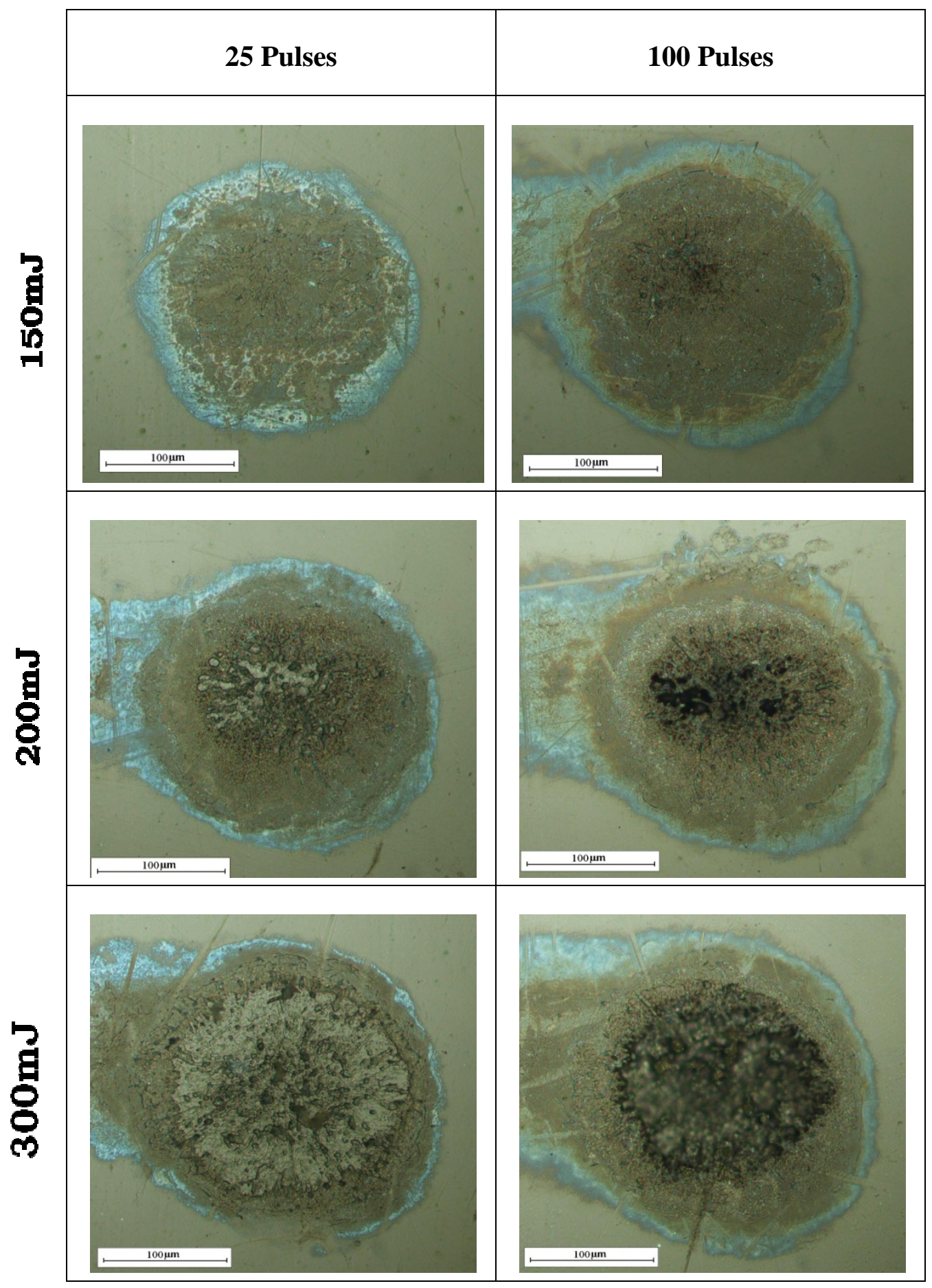

Figure 42 For underwater environment, at 25 to 100 Pulses (5 to 20Hz) optical micrographs (at 20x) showing ablation 


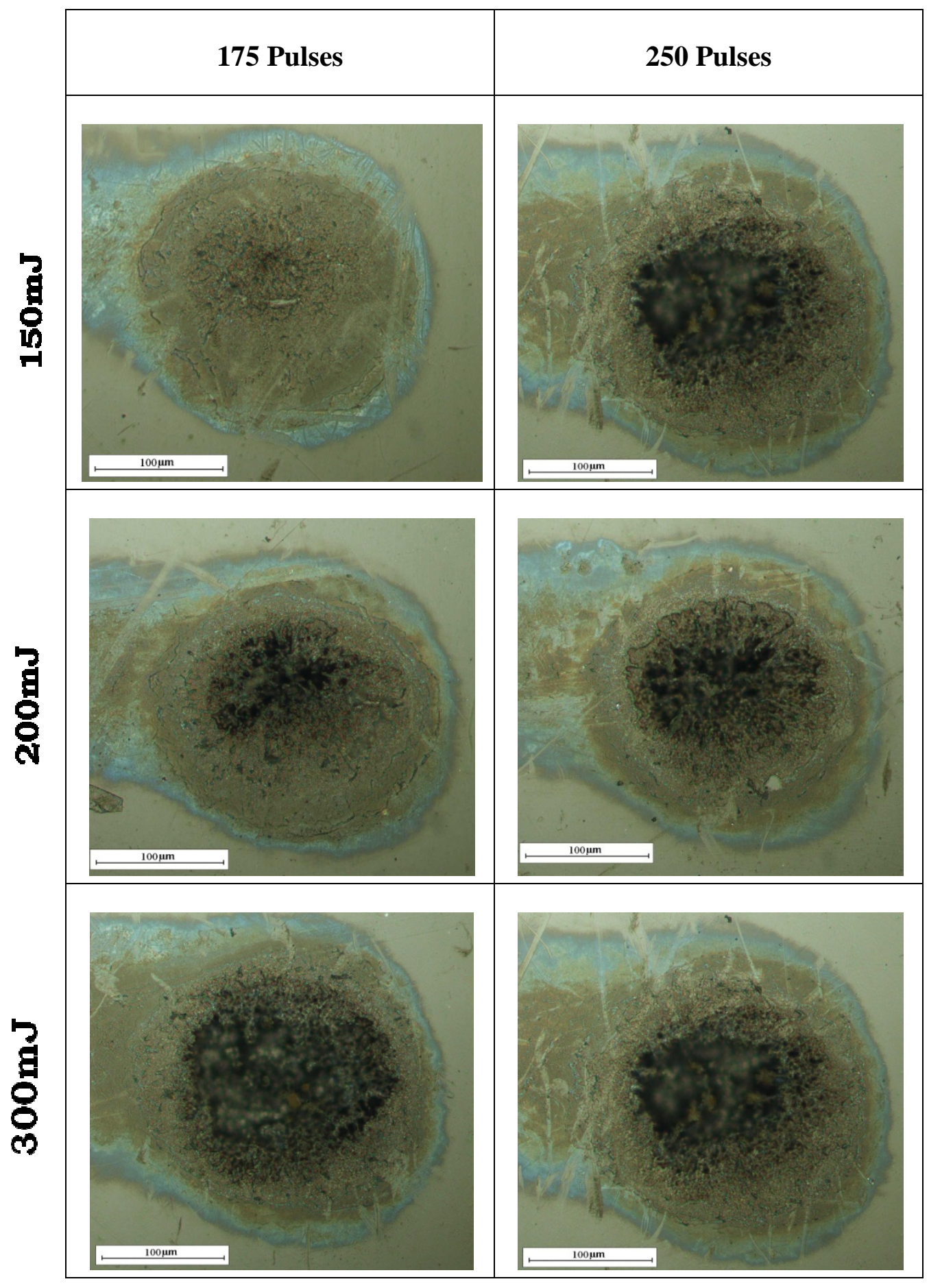

Figure 43 For underwater environment, at 175-to-250 Pulses (35-50Hz) optical micrographs (at 20x) showing ablation 


\subsubsection{Ablation Depth Comparison: Air Vs. Underwater}

The following surface mapping micrographs show ablation characteristics for in air and underwater micromachining on polyurethane samples:

\section{A) At 200mJ @ 25 -250 Pulses}

\begin{tabular}{|c|c|c|}
\hline & In Air & Underwater \\
\hline (25 Pulses) & & \\
\hline & & \\
\hline (100 Pulses) & & \\
\hline (250 Pulses) & & \\
\hline (175 Pulses) & & \\
\hline
\end{tabular}


B) At 300mJ @ 25 -250 Pulses

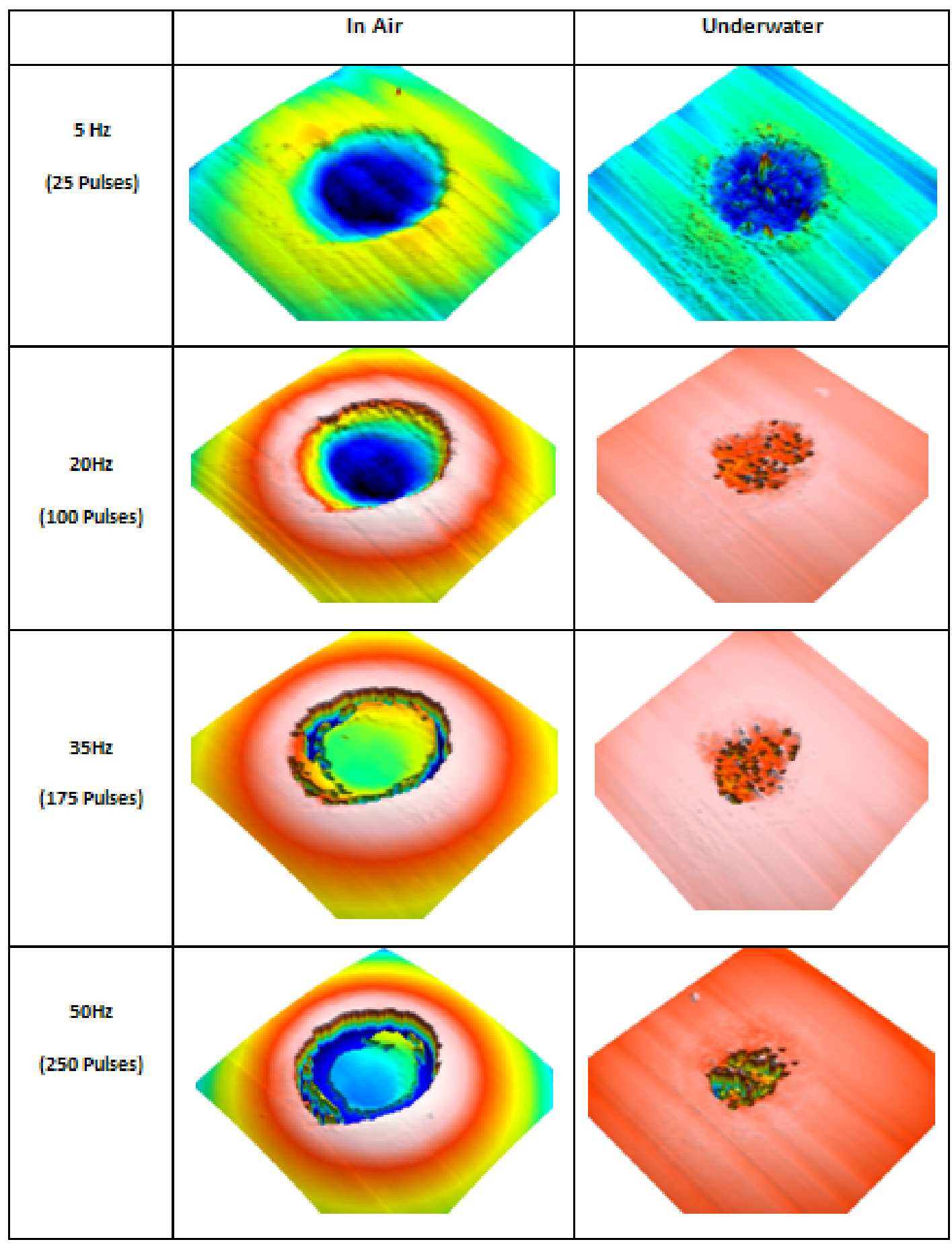




\subsubsection{Depth Profiles in air and underwater}

A) In air, hole depth profiles at 150mJ @ $5 \mathrm{~Hz}-50 \mathrm{~Hz}$ (25 - 250 Pulses)

Line Profile Location
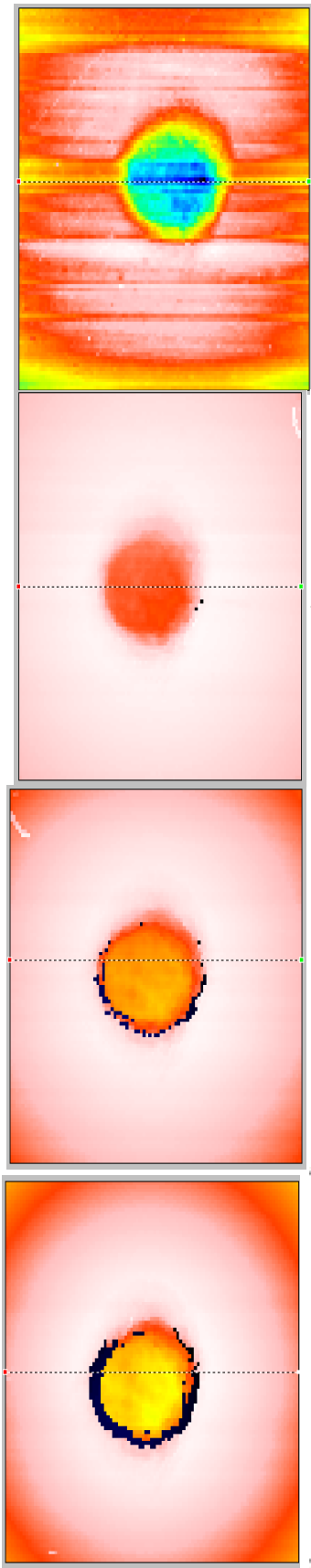
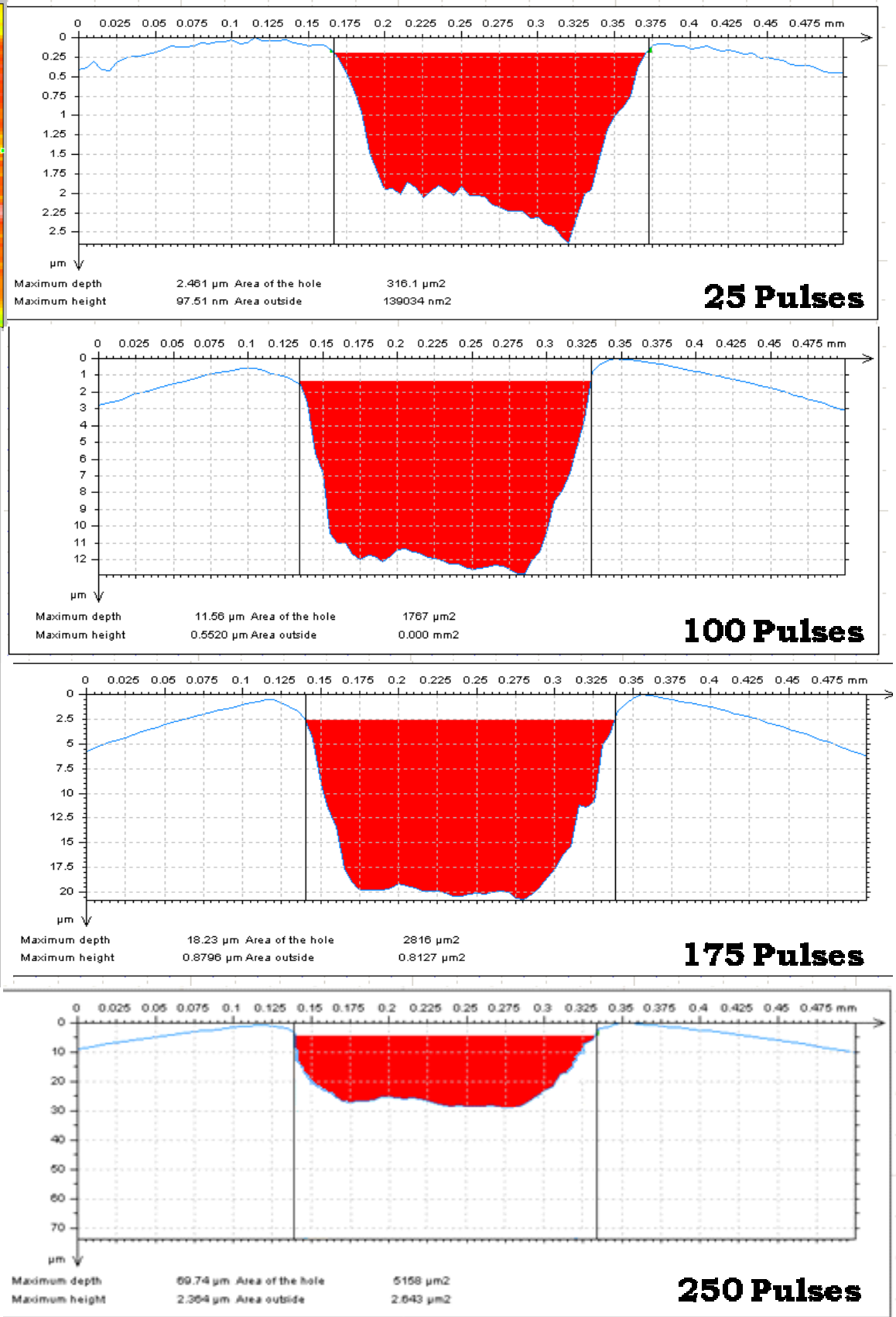
B) In air, hole depth profiles at 200mJ @ $5 \mathrm{~Hz}-50 \mathrm{~Hz}$ (25 - 250 Pulses)

Line Profile Location

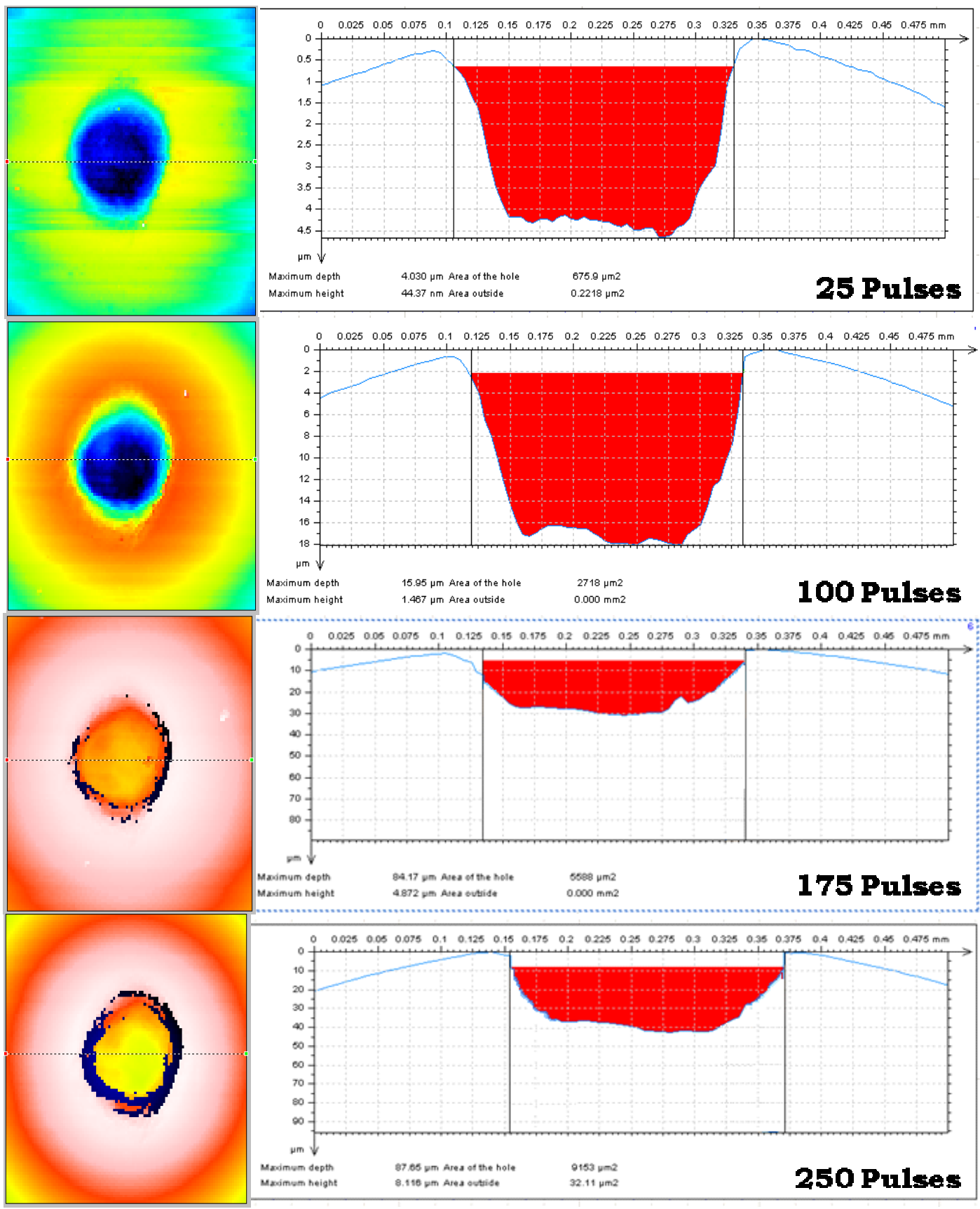


C) In air, hole depth profiles at 300mJ @ $5 \mathrm{~Hz}-50 \mathrm{~Hz}$ (25 - 250 Pulses)

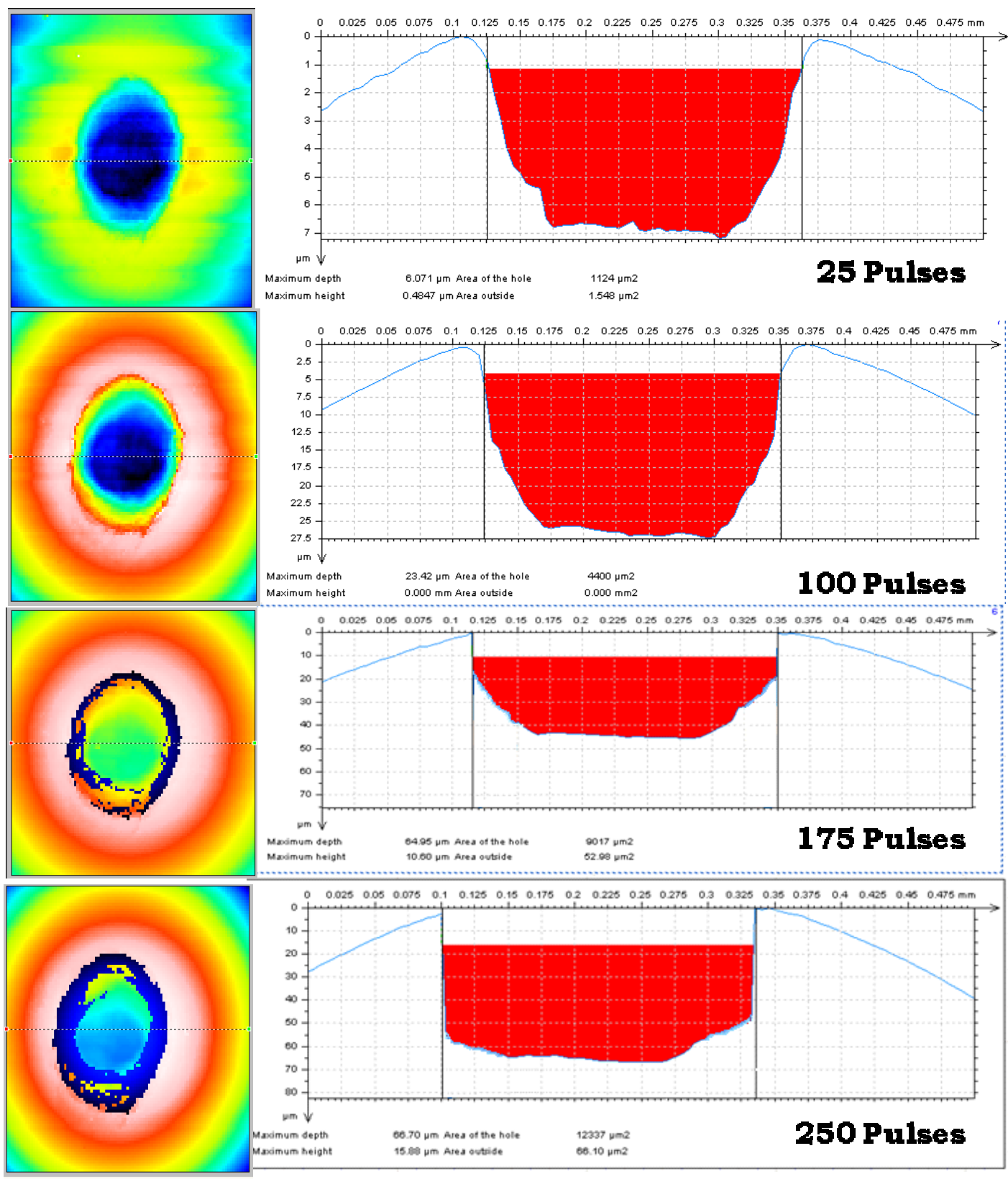


D) Underwater, hole depth profiles at 200mJ @ $5 \mathrm{~Hz}-50 \mathrm{~Hz}$ (25 - 250 Pulses)

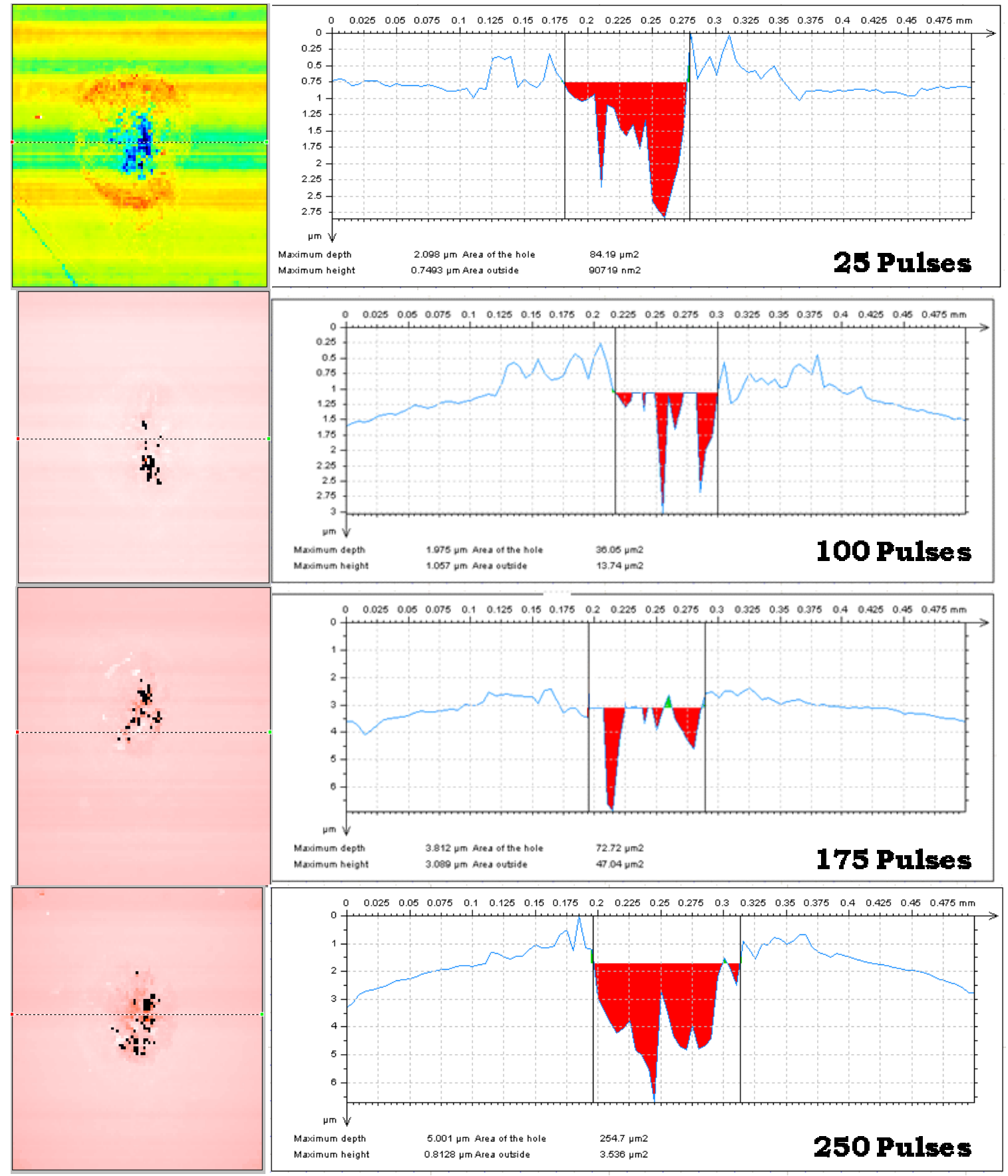


E) Underwater, hole depth profiles at 300mJ @ $5 \mathrm{~Hz}-50 \mathrm{~Hz}$ (25 - 250 Pulses)

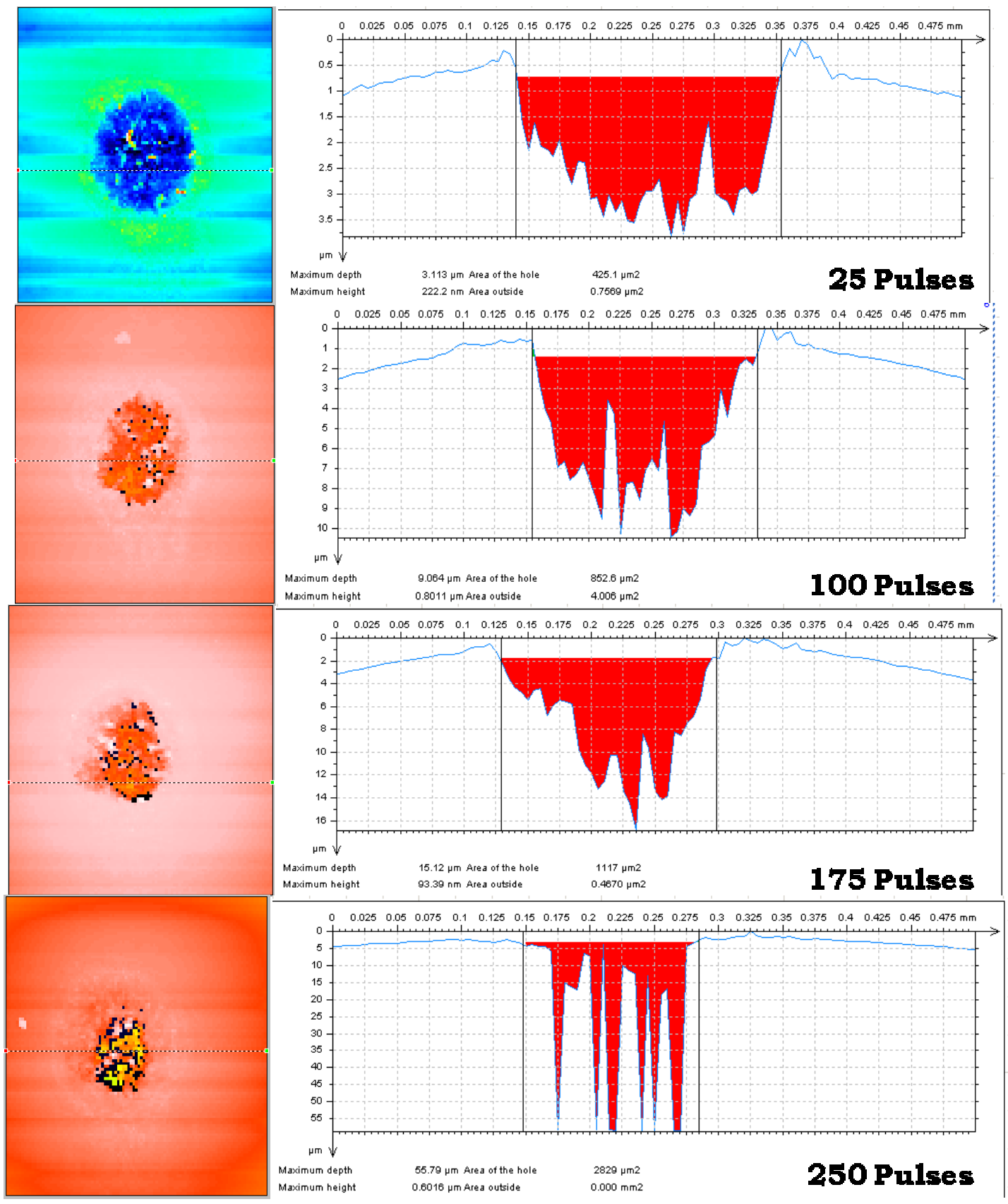


Fig. 44 shows the relationship between etch rate/pulse and input energy of in air micromachining of polyurethane sample:

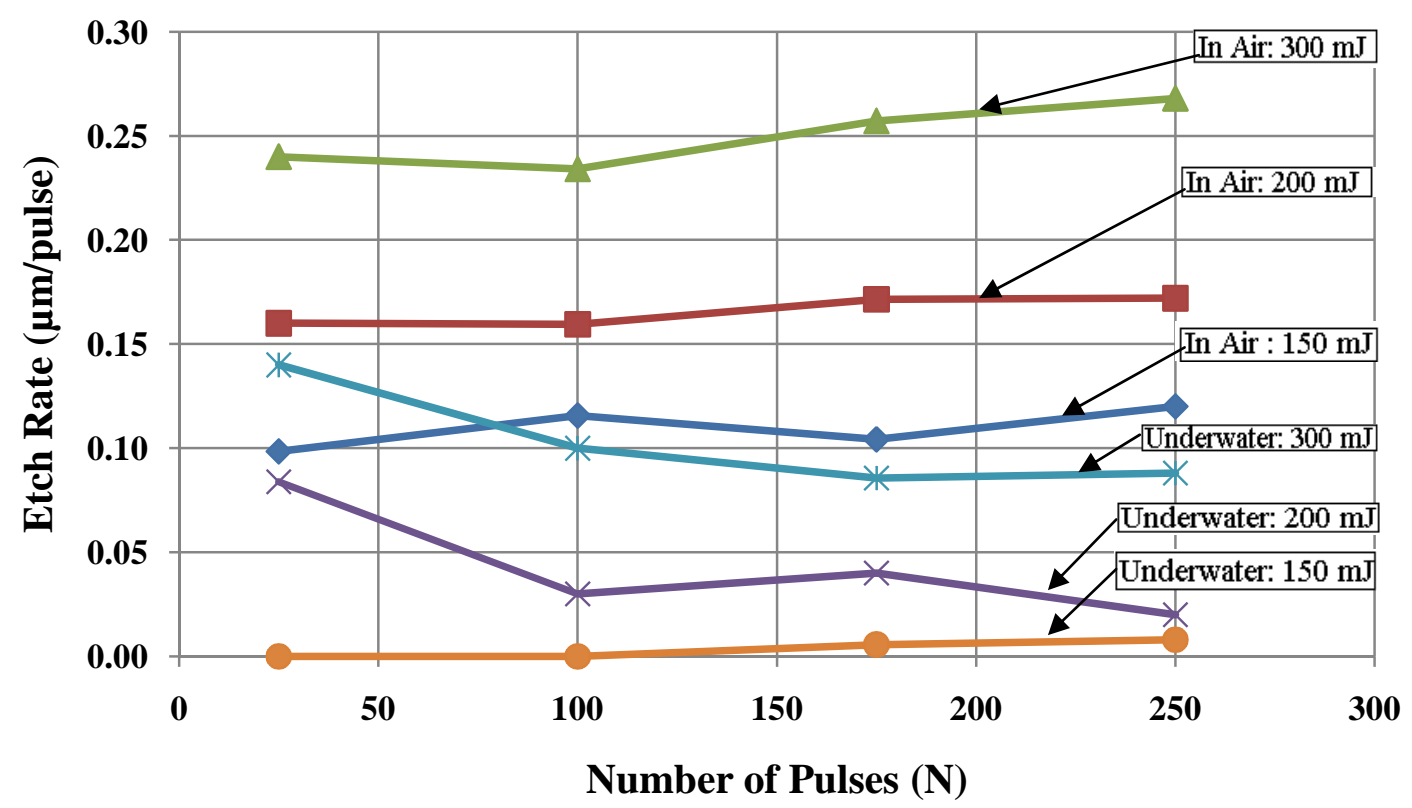

Figure 44 Relationship between etch rate/pulse ( $\mu \mathrm{m} / \mathrm{pulse})$ and number of pulses (N)

The ablation behavior of polyurethane sample $(1 \mathrm{~mm})$ was analyzed by developing relationship between the etch rate/pulse and the number of pulses. From Fig. 45 , it is seen that for in air sample, the etch rate/pulse is $0.18 \mu \mathrm{m} / \mathrm{pulse}$ and for under water sample, etch rate is $0.07 \mu \mathrm{m} /$ pulse. This data is consistent with the etch rate/pulse from Masubuchi et. al (2001). The trend for in air sample indicates a constant etch rate with increase in the number of pulses, where as in underwater sample the etch rate tends to decrease with increase in the number of pulses. Also, as evident from the surface profiler micrographs, the underwater ablated area may not allow direct line of sight for 
the laser beam to reach the bottom of the hole. Furthermore, the presence of water and dynamics of vaporization effects may cause the etch rate to decrease over time.

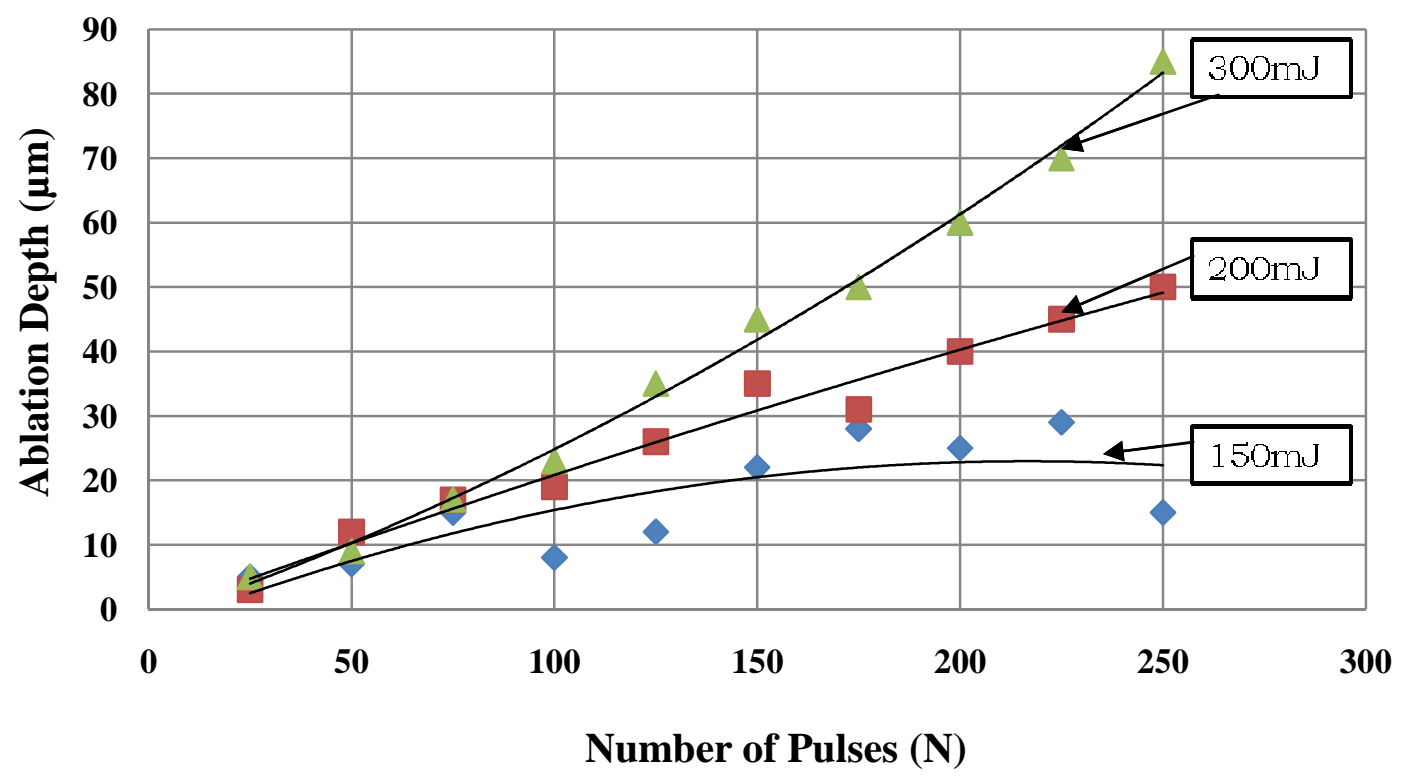

Figure 45 Laser micromachining in air shows variation in ablation depth Vs repetition rates

Fig. 45 shows the variation in ablation depth with the increase in number of pulses $(25-250$ pulses at $5 \mathrm{~Hz}$ to $50 \mathrm{~Hz})$ at pulse energies of $150 \mathrm{~mJ}, 200 \mathrm{~mJ}$ and $300 \mathrm{~mJ}$. With $150 \mathrm{~mJ}$, the curve shows a steady increase of depth value $(\sim 10 \mu \mathrm{m}$ to $20 \mu \mathrm{m})$ at repetition rates from $20 \mathrm{~Hz}$ to $30 \mathrm{~Hz}$. After 175 pulses (at $30 \mathrm{~Hz}$ ) the curve shows a constant trend which implies increase in depth does not proceed further and beyond 30 $\mu \mathrm{m}$ for $150 \mathrm{~mJ}$ pulse energy. With $200 \mathrm{~mJ}$, etch depth data shows a linear increase with increase in number of pulses. The maximum depth of $50 \mu \mathrm{m}$ was observed at 250 pulses $(50 \mathrm{~Hz})$ at $200 \mathrm{~mJ}$ pulse energy. With $300 \mathrm{~mJ}$, the depth trend curve shows a significantly higher slope as compared to $150 \mathrm{~mJ}$ and $200 \mathrm{~mJ}$ curves. High input energy per pulse 
triggers faster ablation of the specimen under normal conditions. A maximum depth of $85 \mu \mathrm{m}$ was observed at 250 pulses $(50 \mathrm{~Hz})$ for $300 \mathrm{~mJ}$ per pulse energy.

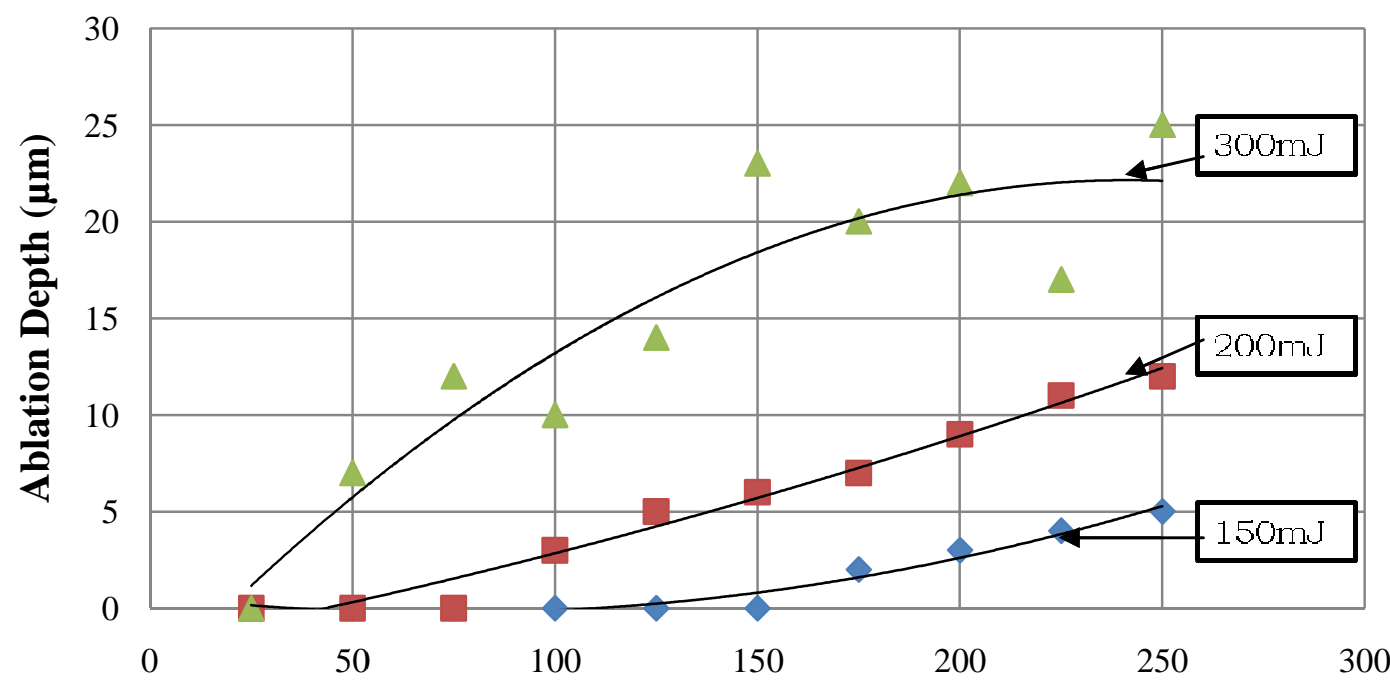

Number of Pulses (N)

Figure 46 Laser micromachining underwater showing variation in ablation depth with increase in number of pulses

For underwater micromachining of polyurethane sample, at $150 \mathrm{~mJ}$ pulse energy

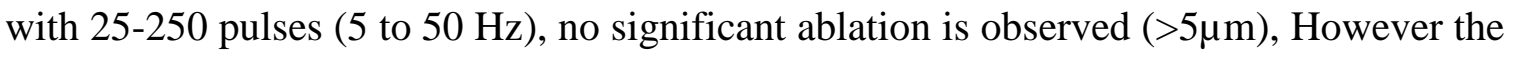
surface of the specimen is affected due to the bombardment of photons. From the literature, it is noted that the ablation phenomenon for polyurethane depends on its primary and secondary structures because the surface of the laser induced specimen shows decomposition of primary structures of polyurethane to its secondary structures due to fast fragmentation but no sign of scattered debris (Suzuki 1998; Masubuchi 2001). From Fig. 46, under $150 \mathrm{~mJ}$ of pulse energy, one of the reason for no ablation could be due to lower repetition rate leaving enough time for the exposed area to cool down before 
the next pulse arrives at the surface. The range of $150 \mathrm{~mJ}$ at 5 to $50 \mathrm{~Hz}$ provides an important observation where ablation first starts, as it is clearly observed from the optical images that the ablation if any started first at the center and proceeded towards the boundaries as the laser fluence or repetition rates were increases. Such a behavior could be due to several factors, such as high incident beam angle $\left(90^{\circ} \mathrm{deg}\right)$, heat dissipation to the surroundings.

At $200 \mathrm{~mJ}$ at $1 \mathrm{~Hz}$, with merely 5 pulses, significant surface fragmentation was observed. At $200 \mathrm{~mJ}$ at $5 \mathrm{~Hz}$ (25 Pulses), the decomposition of surface into primary and secondary structures started, followed by photochemical melting which leads to ablation at the center. The ablation threshold is reached within less than 20 pulses at $200 \mathrm{~mJ} / \mathrm{cm}^{2}$ of the pulse energy per pulse. The change in color around HAZ surrounding the hole geometry suggests heating due to photochemical absorption of the incident laser beam. Moving towards the center of the hole suggesting surface decomposition into primary and secondary structures where primary structure ablates partially and forms secondary structure showing all different structural branches of polyurethane polymer. Maximum ablation depth was seen to be $<12 \mu \mathrm{m}$ at (250 pulses) $50 \mathrm{~Hz}$, which suggests that surrounding water may acts as a cooling medium and thus prevent rapid ablation and contributes to heat dissipation and absorption.

At $300 \mathrm{~mJ}$ at 5 pulses $(1 \mathrm{~Hz})$, appreciable detachment of surface film around the edges was seen due to ejection of surface elements under the effect of high input energy per pulse. At $300 \mathrm{~mJ}$ at 25 pulses $(5 \mathrm{~Hz})$, the laser induced decomposition starts formation of secondary structures of the polyurethane polymer, as reported (Suzuki 1998), whereas 
the behavior under high input energy is observed to be different when compared with lower input energy per pulse experiments $(<200 \mathrm{~mJ}$ or $150 \mathrm{~mJ})$. This could be due to rapid heating and presumably generation of a shock wave of high energy pulses striking the surface and heating it consequently (Suzuki 1998; Masubuchi 2001). The formation of molten droplets can be seen on the surface. At $300 \mathrm{~mJ}$ at 50 pulses $(10 \mathrm{~Hz})$, a maximum depth of $7 \mu \mathrm{m}$ is observed which yields an ablation (etch) rate of 0.14 $\mu \mathrm{m} /$ pulse. At $300 \mathrm{~mJ}$ at 75 pulses $(15 \mathrm{~Hz})$, a maximum depth of $12 \mu \mathrm{m}$ is observed which yields an ablation (etch) rate of $0.16 \mu \mathrm{m} /$ pulse. At $300 \mathrm{~mJ}$ at 150 pulses $(30 \mathrm{~Hz})$, a maximum depth of $23 \mu \mathrm{m}$ was observed which yields an ablation rate of $0.15 \mu \mathrm{m} / \mathrm{pulse}$. Similarly at $300 \mathrm{~mJ}$ at $50 \mathrm{~Hz}$ the maximum depth of $25 \mu \mathrm{m}$ was observed yielding an ablation rate of $0.10 \mu \mathrm{m} /$ pulse. This decrease in ablation rate highlights some important factors which interfere with the ablation process of the polymer, such as ejection of debris, plume generation, and water which enters the ablated area further restricts its ablation. An increase in heat affected zone (HAZ) area is also observed consistent with increase in HAZ diameter measurement.

\subsubsection{Change in Hole Diameter}

From Fig.47, the surface diameter curve trends of all six constant input energies $(150 \mathrm{~mJ}, 200 \mathrm{~mJ}$ and $300 \mathrm{~mJ})$ in air and underwater sample increases with increase in number of pulses. On the contrary, the bottom hole diameter decreases in trend as the number of pulses increase, thus a V cross-section of the hole is expected. 


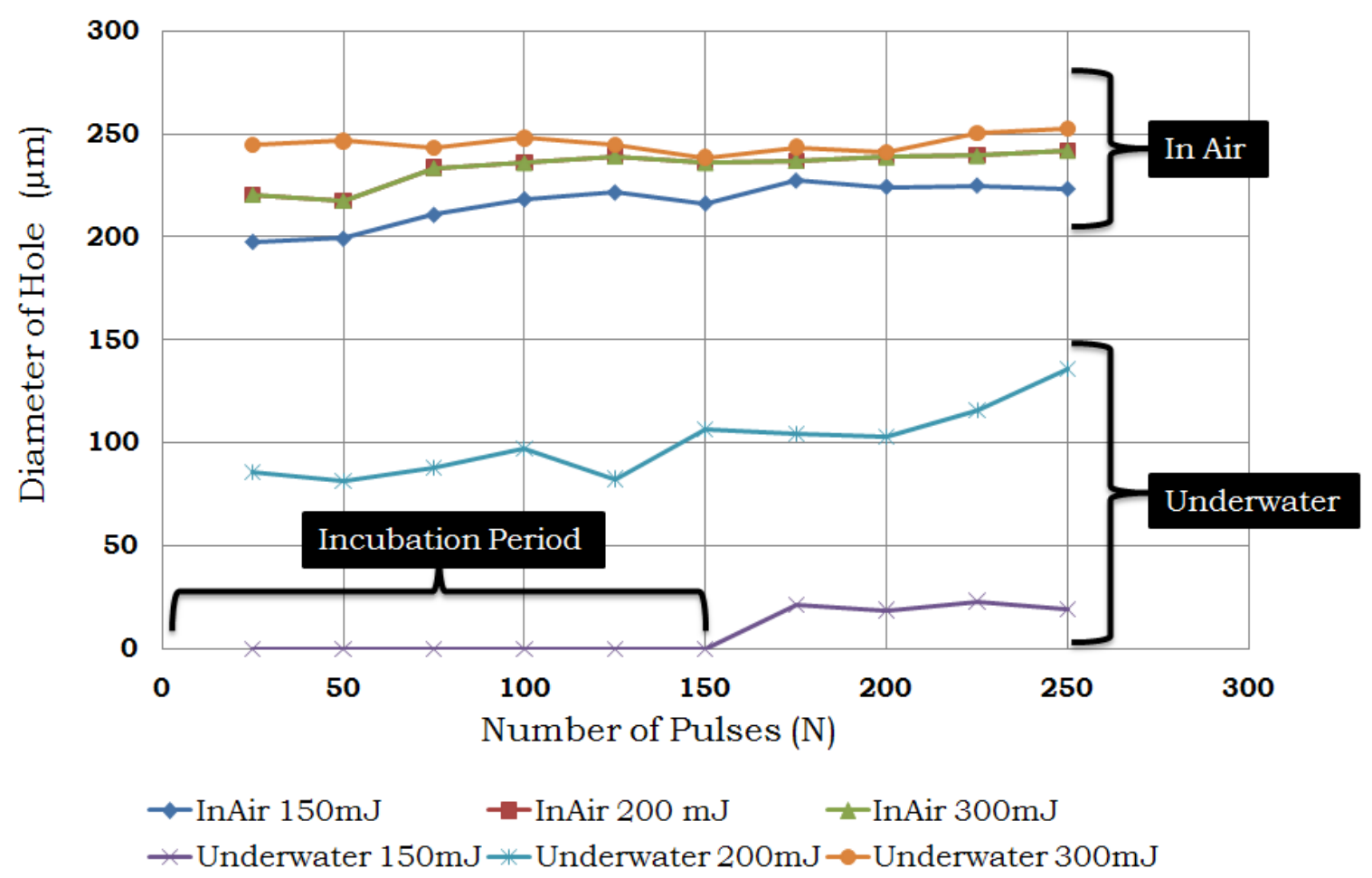

Figure 47 Variation of the diameter of the holes ablated with number of pulses at $150 \mathrm{~mJ}$, $200 \mathrm{~mJ}$, and $300 \mathrm{~mJ}$ for in air and underwater environments

It is important to relate the diameters of the ablated holes with the ablation depth to clearly understand the ablation mechanism. High input energy per pulse triggers faster ablation of the specimen under normal conditions. As seen in Fig.47, for in air samples, the diameter remains constant with increase in the number of pulses. For underwater sample, with $150 \mathrm{~mJ}$ energy, the diameter of the ablation region only appears after 150 pulses which can be termed as incubation period for ablation. With further increase in number of pulses from $150-250$ at $300 \mathrm{~mJ}$ the diameter of the ablation region show similar trend as the air samples. 


\subsubsection{Ablation Mechanism Underwater}

For polyurethane (PU) underwater ablation characteristics differ from in air ablation in a number of ways based on ablation depth, hole diameter, wall angle and surface appearance. From optical micrographs, three distinct surface regions are observed in under water micromachining of the samples; a) Heat Affected Zone (HAZ), b) Thermally Damaged Zone (TDZ) and c) Droplet Zone (DRZ).

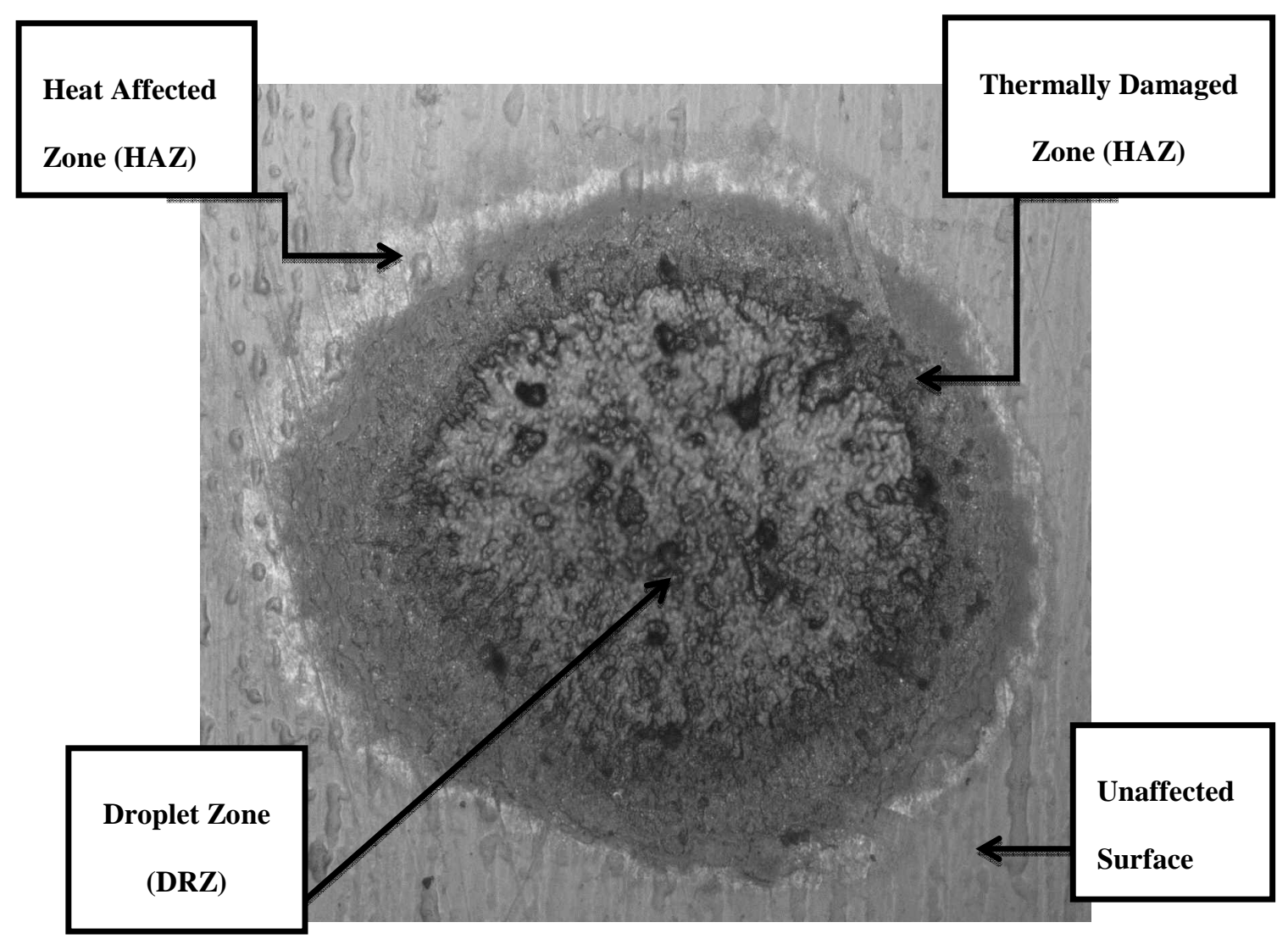

Figure 48 Underwater ablation features on polyurethane sample ablated with 250 pulses @

\section{$500 \mathrm{~mJ}$}

Heat Affected Zone (HAZ) shows minor changes in the topography which implies an increase in surface temperature due to possible reaction of water molecules with 
polymer during ablation under water. Change in color of the HAZ area implies a chemical reaction of polymer's surface with the water molecules. Thermally Damaged Zone (TDZ) shows significant change in the surface roughness as compared to HAZ. Energy insufficient to ablate the surface due to cooling effect of the water gets absorbed into the surface and forms excessively visible detachment of the surface elements of polymer. Thus, surface topography gets affected under the influence of high incident energy. Droplet Zone (DRZ) is the central zone during the micromachining process where the small droplets begin to form as the input energy reaches the ablation threshold of the polyurethane (PU) polymer. Ablation in this zone is intermittent and discontinuous. The diameter of HAZ is observed to be larger than TDZ and DRZ, Similarly, the surface diameter of TDZ is observed to be larger than DRZ.

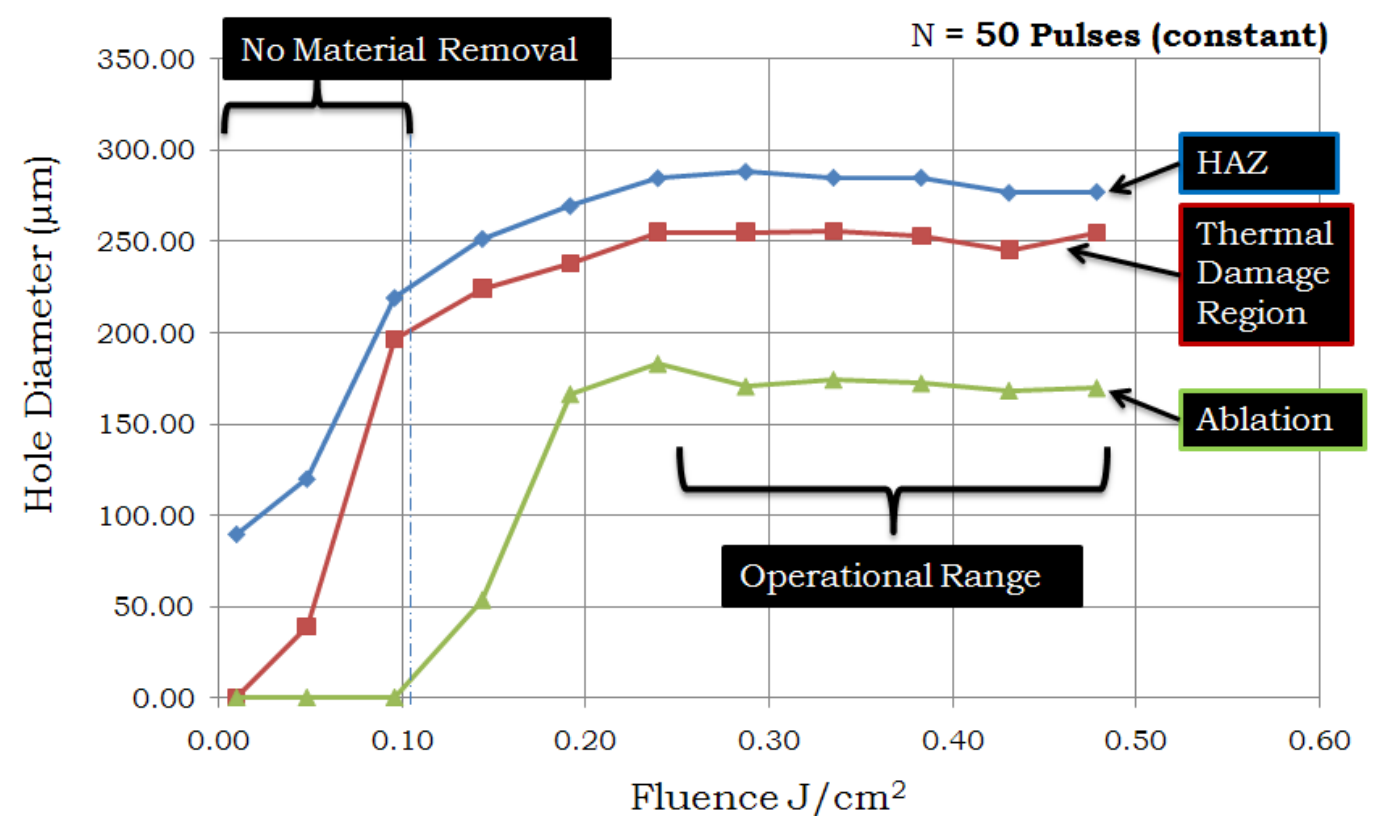

Figure 49 For underwater micromachining, change in HAZ, TDZ and DRZ diameter of polyurethane sample 
From Fig.49, following observations are made for all three zones and curve trends:

- At a fluence of $0.01 \mathrm{~J} / \mathrm{cm}^{2}$, Heat affected zone diameter is measured to be $220 \mu \mathrm{m}$, whereas DRZ do not exist at this point.

- During steps $0.01 \mathrm{~J} / \mathrm{cm}^{2}$ to $0.20 \mathrm{~J} / \mathrm{cm}^{2}$ the diameter of HAZ increases linearly with respect to increase in input energy. During steps $0.05 \mathrm{~J} / \mathrm{cm}^{2}$ to $0.14 \mathrm{~J} / \mathrm{cm}^{2}$, the DRZ begins to increase in diameter with its slope remains parallel to the slope of TDZ diameter.

- During steps from $0.14 \mathrm{~J} / \mathrm{cm}^{2}$ to $0.24 \mathrm{~J} / \mathrm{cm}^{2}$, a rapid increase in slope and diameter of the DRZ is observed. The average increase of DRZ diameter and its slope is observed to be significantly higher as compared to the average increase of diameter/slope for TDZ and HAZ zones.

- During $0.29 \mathrm{~J} / \mathrm{cm}^{2}$, the diameters of all three different zones remain constant in value, thus can be considered as ablation threshold.

- The energy required for each zone to start its formation is observed as HAZ at 0.0 $1 \mathrm{~J} / \mathrm{cm}^{2}$ for $220 \mu \mathrm{m}$, TDZ at $0.05 \mathrm{~J} / \mathrm{cm}^{2}$ for $40 \mu \mathrm{m}$ and DRZ at $0.14 \mathrm{~J} / \mathrm{cm}^{2}$ for $50 \mu \mathrm{m}$. The ablation of the specimen was observed after a fluence of $0.25 \mathrm{~J} / \mathrm{cm}^{2}$.

Although all three zones play an important role in ablation of polyurethane in underwater micromachining, ablation starts only when droplet zone (DRZ) is formed under a given set of input parameters, e.g. Ablation $>\mathrm{E}=200 \mathrm{~mJ}, \mathrm{~Hz}=10 \mathrm{~Hz}$, Pulses $=$ 50. The consistency of results for diameters of different zones is realized by comparing data to similar experiments. With a constant well defined increase in input energy $(10 \mathrm{~mJ}$ per step) in the experiment the rate change of diameter for each three zones (HAZ, TDZ, 
DRZ) changes independently to each other. HAZ diameter was observed to be having minimum rate of change $(<45 \mu \mathrm{m})$ for each consecutive step in experiment. TDZ diameter was observed to be showing maximum change $(>85 \mathrm{um})$ in diameter during the incubation period for DRZ. The droplet zone (DRZ) diameter was observed to be having significantly higher change as compared to HAZ but lies in between HAZ and TDZ. The overall trend for diameter change stabilizes as the input energy parameter is increased beyond $300 \mathrm{~mJ}$. 


\subsection{Effect of Taper Angle on Ablation}

Another important part of the research was to investigate the effect of taper angle on the ablation behavior of polyurethane samples and establish a relationship of taper angle behavior and ablation depth. The hole diameter at the surface, and diameter at the bottom of the hole and the depth of the holes using focus variation method using an optical microscope are used to calculate the taper angle. Fig. 50 explains various components included in the calculations for taper angle while assuming beam incident angle to be a right angle:

\section{Required Input: \\ - Value of $x$ \\ - Value of R1 \\ - Value of R2}

\section{Calculations for:}

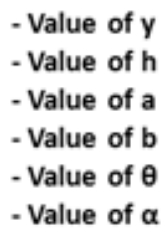

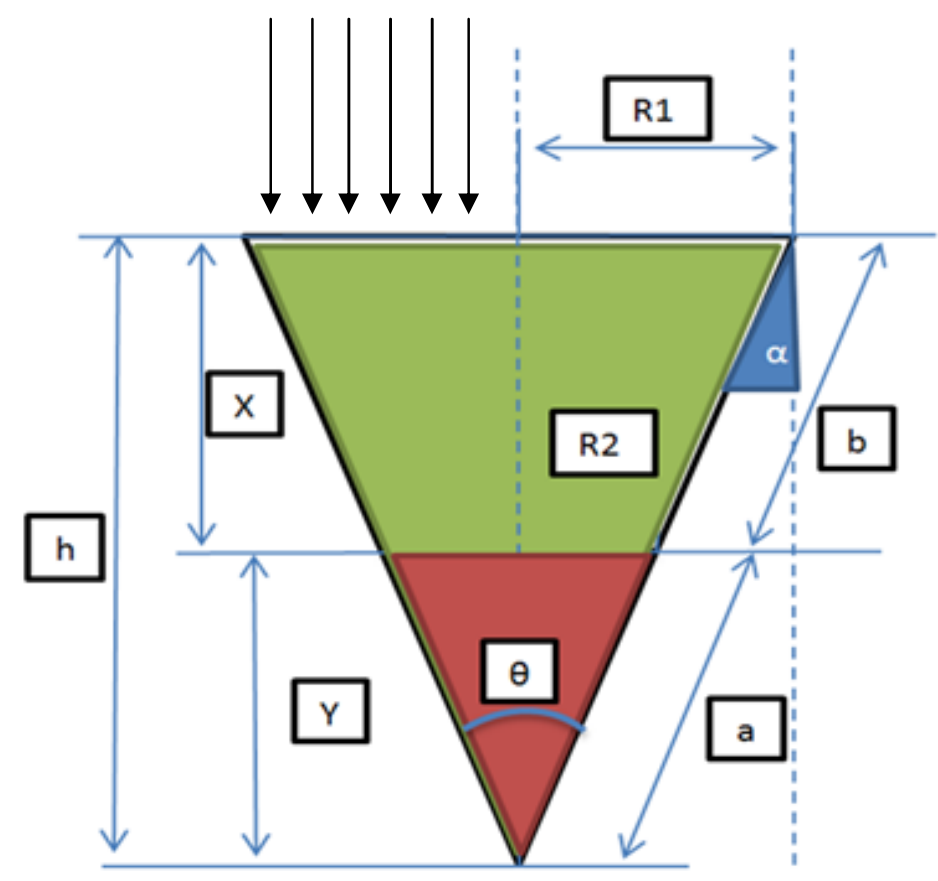

Figure 50 A illustration of the hole geometry cross section for calculating taper angle for hole walls 
Equations (8.1 to 8.8) provide the formula for the calculation of taper angle for underwater and in air micromachining of polyurethane samples:

$$
\text { Theoretical } Y=\frac{(X \times R 2)}{(R 1-R 2)}, \quad \text { Theoretical } h=X+Y
$$

$$
\text { Theoretical } a=\sqrt{Y^{2}+R 2^{2}}
$$

$a+b=\sqrt{(X+Y)^{2}+R 1^{2}}, b=(a+b)-a$

$$
\mathbb{V}=\operatorname{Cos}^{-1}\left(\frac{R 1}{(a+b)}\right) \times\left(\frac{180}{\pi}\right)
$$

$$
\text { side angle }(\omega)=180-(2 \times \mathbb{V}) \text {, }
$$

$$
\text { Cone Angle } \theta=\left(\frac{\pi \times R 2}{a}\right) \times\left(\frac{180}{\pi}\right)
$$

$$
\text { Taper Angle }(\alpha)=\frac{\omega}{2}
$$

Volume Removed $(v)=\left(\left(R 1^{2}\right)+(R 1 \times R 2)+(R 2)^{2}\right) \times\left(\pi \times h \times\left(\frac{1}{3}\right)\right)$

NOTE: Using above equations it is possible to calculate the taper angel for non-through holes.

It is important to note here that all the ablated holes were not through holes since it would require longer exposure time and higher input energies to have a through hole. 
Longer exposure times for polyurethane cause excessive heat affected zones and very poor edge geometry. Also, due to the nature of the polyurethane (PU) polymer, it becomes difficult to obtain cross sectional measurement of ablated hole depth profile. Thus, the equations employed to calculate the taper angle, volume removal, depth, surface and bottom diameters give very useful information about ablation phenomenon of polyurethane (PU) polymer. Figs 51-54 show the plots of the taper angles variation in air and underwater ablation at pulse energies of $150 \mathrm{~mJ}, 200 \mathrm{~mJ}$, and $300 \mathrm{~mJ}$ :

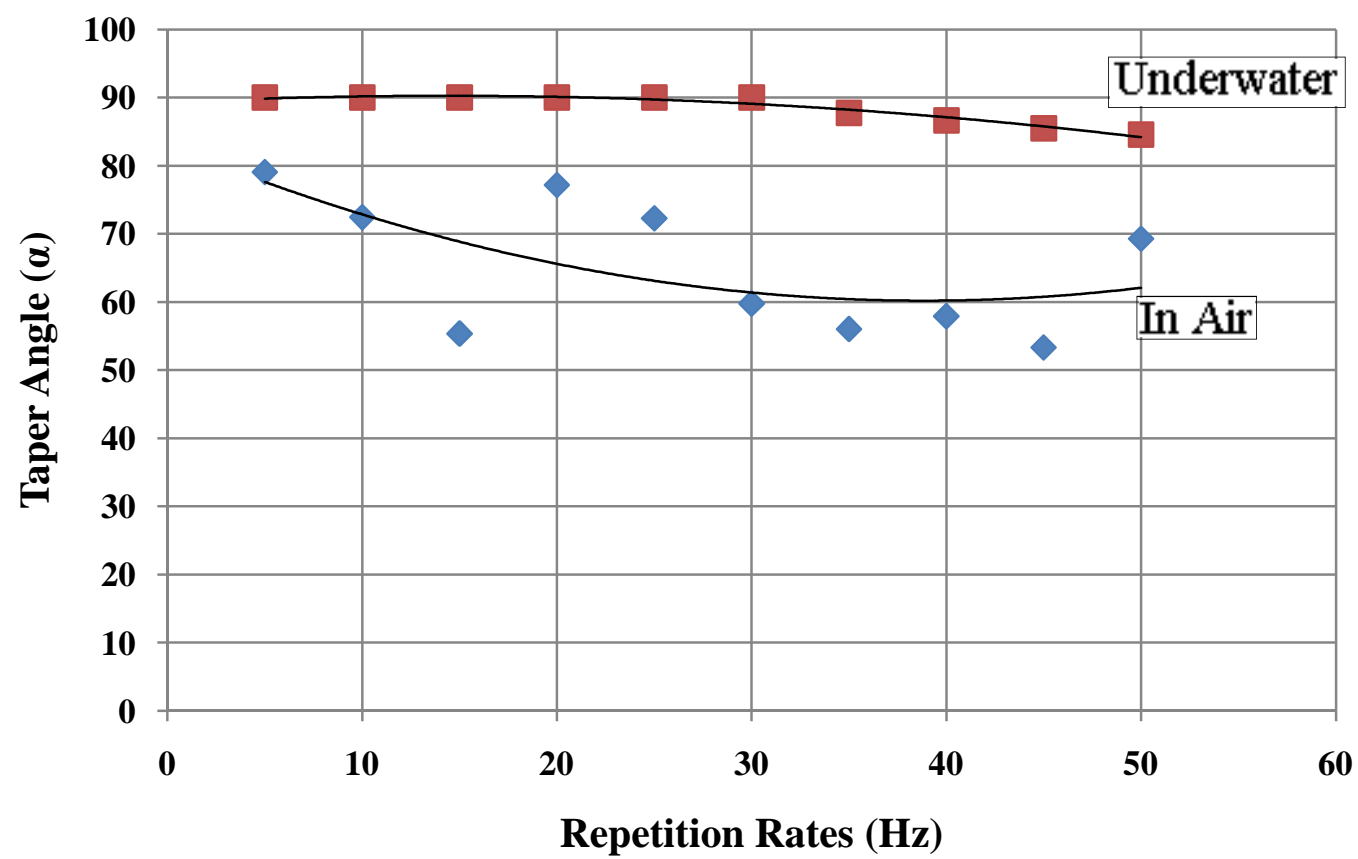

Figure 51 Variation of taper angle with repetition rates for $150 \mathrm{~mJ}$ pulse energy for in air and underwater 


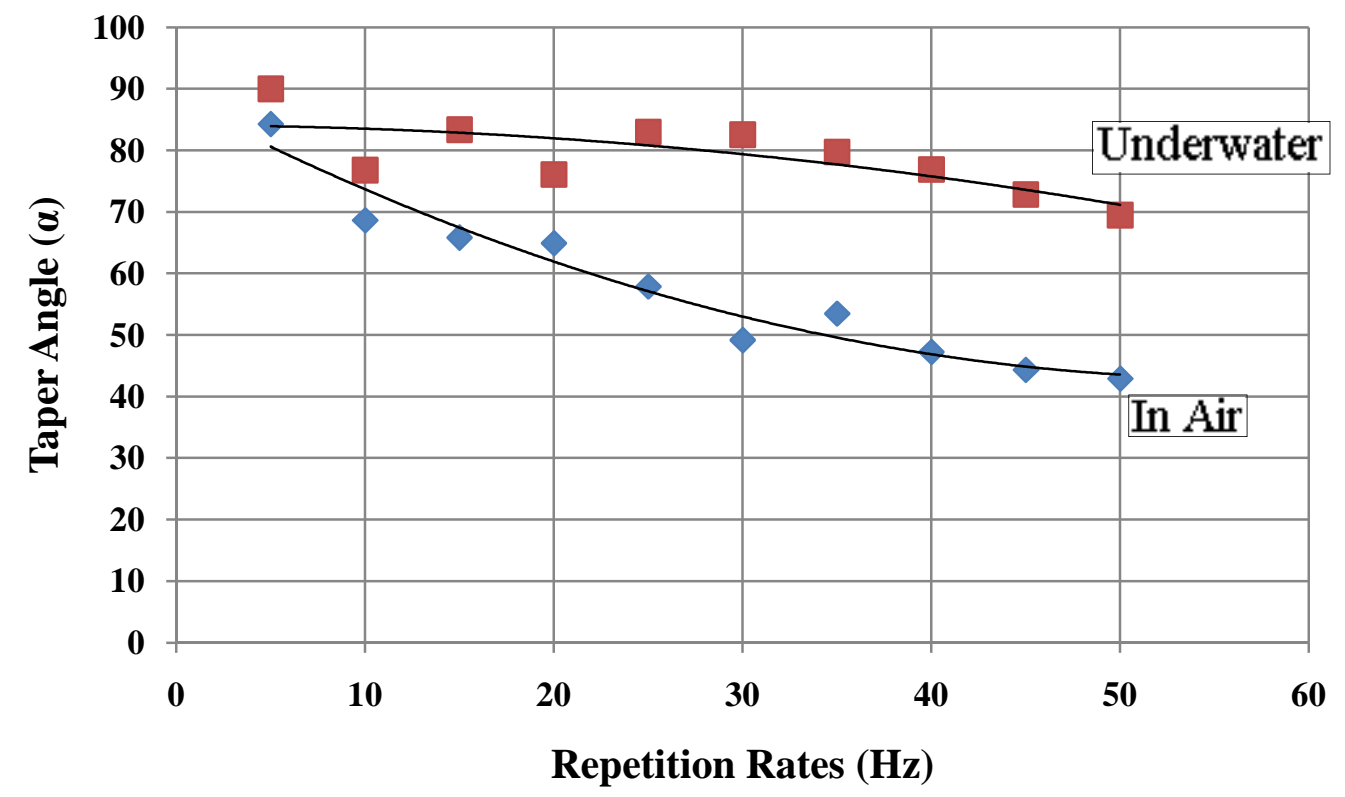

Figure 52 Variation of taper angle with repetition rates for $200 \mathrm{~mJ}$ pulse energy for in air and underwater

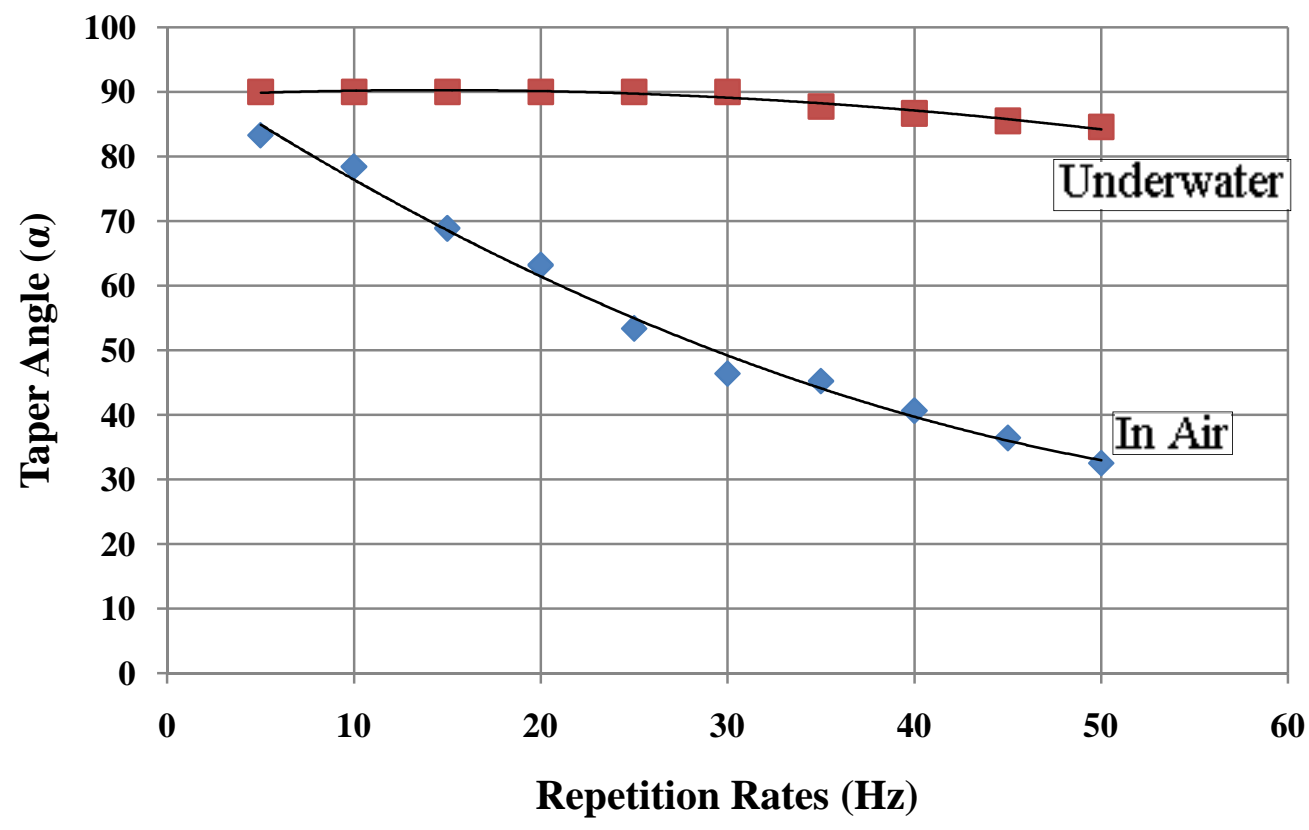

Figure 53 Variation of taper angle with repetition rates for $300 \mathrm{~mJ}$ pulse energy for in air and underwater 
From Figs. 51 to 54 , high taper $\left(90^{\circ}\right)$ angles are obtained at lower input energy $(150 \mathrm{~mJ})$ with low repetition rates $(25$ pulses or $5 \mathrm{~Hz})$ where the ablation depth is found to be $<2 \mu \mathrm{m}$. A maximum depth of $105 \mu \mathrm{m}$ is reported when input parameters are increased to $300 \mathrm{~mJ}$ at 250 pulses $(50 \mathrm{~Hz}$ ). Larger heat affected zone (HAZ) is also observed at these high input parameters.

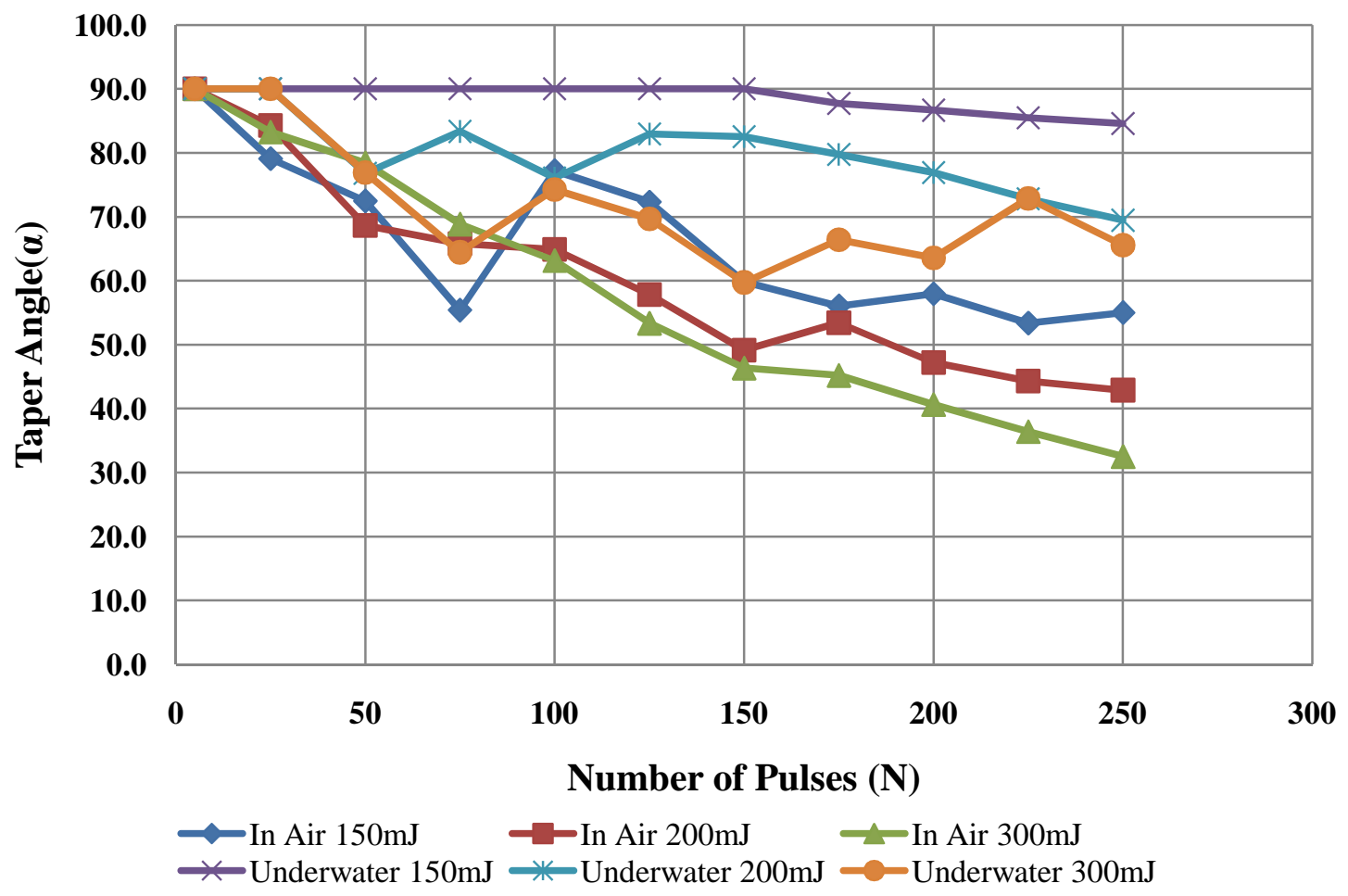

Figure 54 Taper angle comparisons between in air and underwater ablation with increase in number of pulses

Lower taper angles were observed when high input energy $(300 \mathrm{~mJ})$ is used with higher repetition rates $(250$ pulses or $50 \mathrm{~Hz}$ ) which imply rapid ablation of polyurethane specimen. The edge geometry of the hole was observed to be coarse in appearance while 
working at such high input parameters. However, while using $300 \mathrm{~mJ}$ at 150 pulses (30 $\mathrm{Hz})$ as input parameters the edge geometry is observed to be fine with less appearance of HAZ. At $300 \mathrm{~mJ}$ at 150 pulses $(30 \mathrm{~Hz})$, the taper angle was found to be $\sim 50^{\circ}$ whereas the lower taper angle observed is $\sim 30^{\circ}$ at $300 \mathrm{~mJ} / \mathrm{cm}^{2}$ at 250 pulses $(50 \mathrm{~Hz})$. In accordance with previous results, a high taper angle of $\sim 75^{\circ}$ is observed at $300 \mathrm{~mJ}$ at 25 pulses (5 $\mathrm{Hz}$ ), the taper angle is observed to be increasing. The maximum depth at this point is observed to be $5 \mu \mathrm{m}$. Lowest taper angle of $\sim 57.31^{\circ}$ is observed at $50 \mathrm{~mJ}$ at 250 pulses (50 $\mathrm{Hz}$ ), the maximum depth at this point is observed to be $30 \mu \mathrm{m}$.

Above observations highlight an important fact that the input energy parameter should be given priority over repetition rates if maximum depth is to be achieved. This observation along with previous test suggests maximum ablation takes place only with low taper angles coupled with high input energies and low repetition rates. When both ranges were plotted against each other the curves intersect at a common point, the axis values for this were calculated to find the optimal operational input parameters as : Taper Angle $=\sim 60^{\circ}$ (deg.) @ E=255 mJ @ HZ=25 Hz. Employing above input parameters results in fine edge geometry, minimized heat affected zone and maximum depth values. Furthermore, it helps in prediction of maximum depth, volume removal, and major/minor diameters without hurting the geometry of the pattern profile.

\subsubsection{Results and Discussion}

Following important conclusion are made to compare in air ablation versus underwater ablation of polyurethane samples: 
- For in air ablation process of polyurethane only the bottom diameter of the hole always decreases with increase in repetition rates or fluence. This suggests a tapered cross-sectional profile of the hole geometry.

- For underwater ablation of polyurethane specimen, when input energy is increased beyond $300 \mathrm{~mJ}$, the droplet zone diameter decreases which imply rapid ablation or vaporization of the surface.

- For both in air and underwater ablation process of polyurethane specimen the HAZ, TDZ and DRZ diameters tends to increase as the repetition rate/input energy was increased.

- High taper angles were estimated for underwater micromachining when the input energy was kept below 150mJ, but much lower taper angles were estimated in case of in air micromachining. Upon increase of input energy to $300 \mathrm{~mJ}$, the underwater taper angles remained higher than in air.

- Maximum ablation depths and lowest taper angles were observed when the specimens were micro-machined in air.

- No through holes where observed in both in air and underwater specimens due to high thickness of the sample $(1 \mathrm{~mm})$.

An attempt was made to produce a through hole, However this led to large heat affected zones for both in air and underwater specimens but in air sample showed higher ablation depth. For underwater specimen, the resistance to ablation and large HAZ due to the presence of water is observed as a dominant reason for resulting topography and minor ablation depth. For underwater specimens, the diameter of ablated hole is very 
small $(120 \mu \mathrm{m})$ as compared to in air specimen where diameter is observed to be $(300 \mu \mathrm{m})$. However, unlike in air specimens, the thermally damaged zone for underwater specimens showed high surface roughness values. High optical magnification showed entrapment of ejected decomposition products due to the interaction of pulsed excimer laser irradiation leading to ablative photo-decomposition.

\subsection{Two-dimensional (2D) Pattern Generation}

After determination of input parameters, mask size, step size, lead, tail and fluence, for achieving best suitable ablation rates and surface characteristics, the approach for achieving a 2D pattern is pursued for polyurethane and compared with similar polymer samples. The laser micromachining and patterning of the polymers is fairly complex due to its dependence over several factors such as absorption coefficient, reflectivity of specimen, environment, beam focal point, ablation characteristics of the polymers etc. Substrates are chosen as polyurethane (PU) and polymer sheets. While the incident laser beam location is fixed, the workpiece is moved in relation to laser beam spot using PSO/G codes, the method of line machining was used to move sample workpiece from point $\mathrm{A}$ to point $\mathrm{B}$, however no Z-axis movement was utilized. Observations are made for seam quality, seam thickness, ablation depth, heat affected zone and debris formation. Thus a preliminary test is conducted to find out suitable feed (f) parameters required for high quality micromachining. Following optical images show the effect of changing feed rates and step size differences on a sample substrate: 


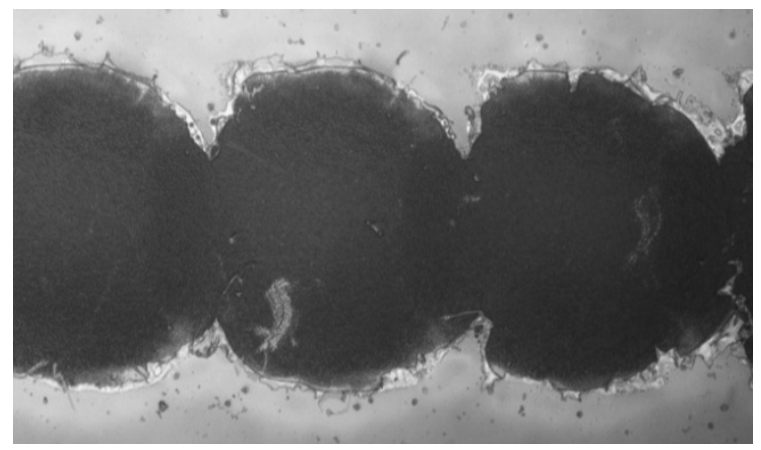

$\mathrm{f}=90 \mu \mathrm{m} /$ pulse

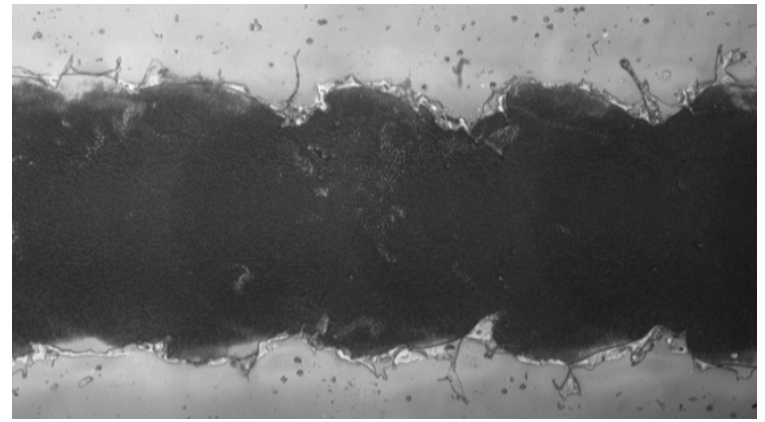

$\mathrm{f}=\mathbf{4 0} \mu \mathrm{m} / \mathrm{pulse}$

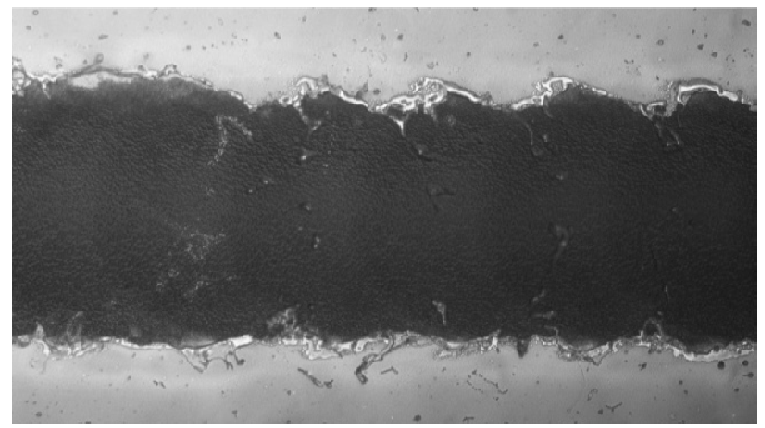

$\mathrm{f}=\mathbf{1 0} \mu \mathrm{m} / \mathrm{pulse}$

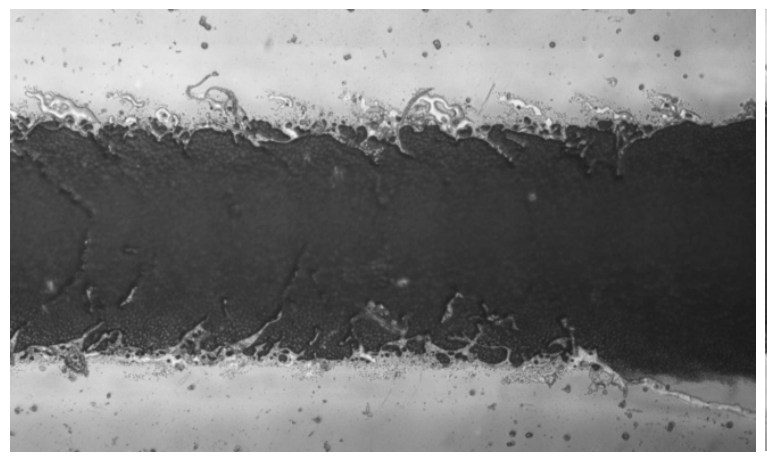

$\mathrm{f}=\mathbf{2} \boldsymbol{\mu \mathrm { m }} / \mathrm{pulse}$

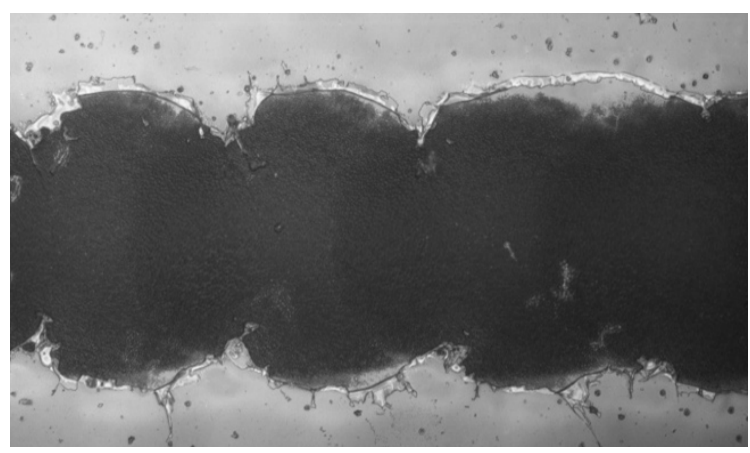

f = $50 \mu \mathrm{m} /$ pulse

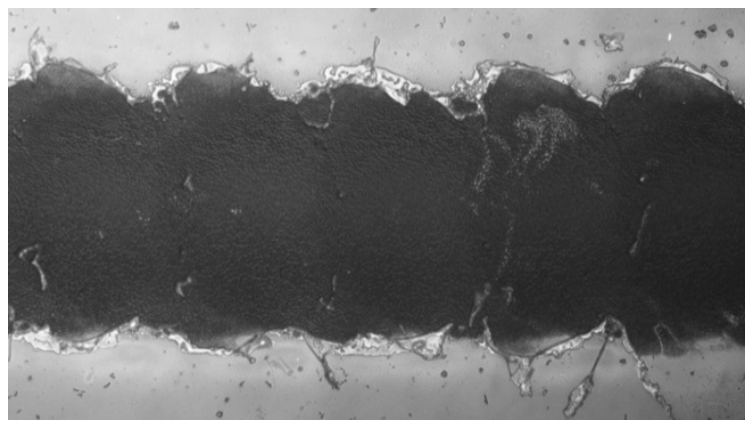

$f=20 \mu \mathrm{m} /$ pulse

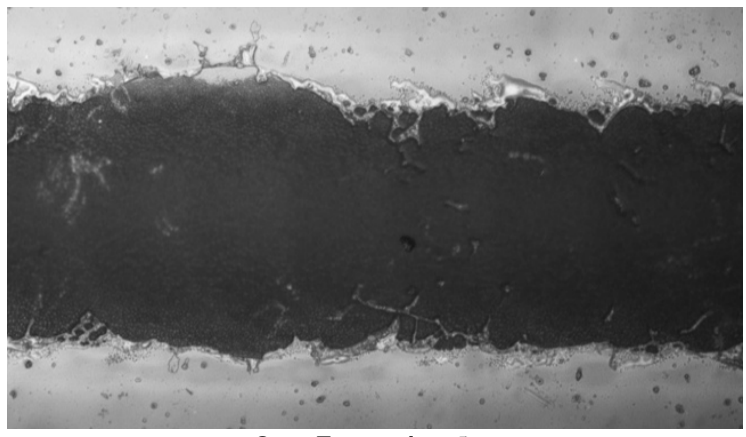

$\mathrm{f}=\mathbf{5} \boldsymbol{\mu \mathrm { m }} / \mathrm{pulse}$

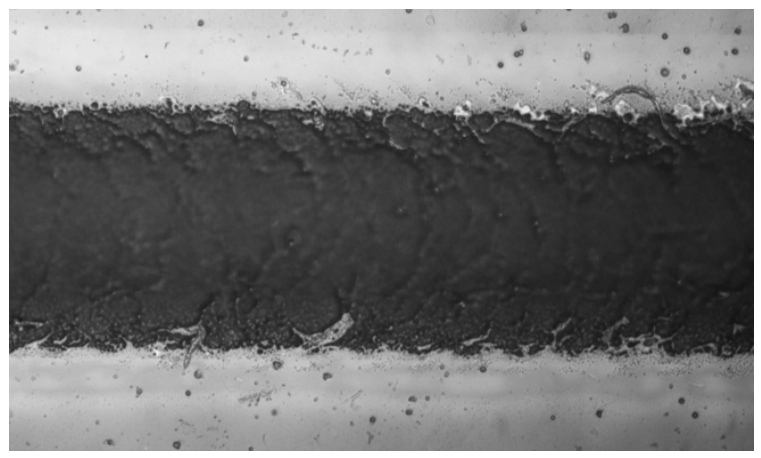

$$
\mathbf{f}=\mathbf{1} \boldsymbol{\mu m} / \text { pulse }
$$

Figure 55 Effect of feed rate $(\mu \mathrm{m} / \mathrm{pulse})$ on seam quality 
From this preliminary test and based on the optical micrographs shown in Fig. 55, formation of ripple like structures is found due to large interval between pulses and feed rates, However, the ripple structures disappear upon increasing the input energy. For feed rates above $90 \mu \mathrm{m} /$ pulse, the seam quality is rather poor and debris is seen around the edges of the seam. Similar debris splashes are observed at 80, 70, 60, 50, 40 and 20 $\mu \mathrm{m} /$ pulse. For feed rates above $50 \mu \mathrm{m} /$ pulse, the seam quality is observed to be poor as compared to shorter feed rates, e.g. $5 \mu \mathrm{m} /$ pulse or $10 \mu \mathrm{m} /$ pulse. However, the heat affected zone is greatly reduced. For feed rates below $5 \mu \mathrm{m}-15 \mu \mathrm{m} /$ pulse, the seam quality is observed to be improved but heat affected zone is also increased; this can be due to the heat coming from ejected decomposition products and or due to a well-known "Fresnel Effect" while laser beam passes through the mask.

\subsubsection{Effect of Mask Size}

Scaling of the pattern can be employed to reduce the actually size of the pattern to its $50 \%, 25 \%, 15 \%$ or $5 \%$ of the original size. For scaling of the pattern, the mask size used in laser optics should also decrease consistently with percentage reduction in pattern size. Different mask sizes can be utilized to form smaller thickness seams provided that correct step distance is utilized. Smaller mask sizes require higher input energies to gain the desired ablation depth. Seam quality is greatly increased when the step size is reduced in combination with increased repetition rates. Seam quality can be improved using low input energy with high repetition rates, However, due to limitations of maximum input parameters as $500 \mathrm{~mJ}$ at $50 \mathrm{~Hz}$, the micromachining is mostly done at around $300-400 \mathrm{~mJ}$ at $30-50 \mathrm{~Hz}$. Selection of feed rate also plays an important role in seam quality; feed 
rates from $1 \mu \mathrm{m}$ to $100 \mu \mathrm{m} /$ pulse are used to find the best seam quality and acceptable performance with other input parameters. A sample pattern shown in Fig. 58 is etched on to the surface of the polyurethane specimen using a particular mask size $(555 \mu \mathrm{m}$, $1152 \mu \mathrm{m})$ at constant input energy $(250 \mathrm{~mJ})$ and repetition rate $(25 \mathrm{~Hz})$. With $1152 \mu \mathrm{m}$ mask size, the width or thickness of the seam was observed to be $270 \mu \mathrm{m}$, along with 42 $\mu \mathrm{m}$ of heat affected zone. No cracking/distortion is observed at the edges. Seam quality greatly improves if small step size distance is used; at round corners the transition was smooth with no sign of splashed debris. Fig 57 shows a surface micrograph of the sample electric pattern shown in Fig. 56.
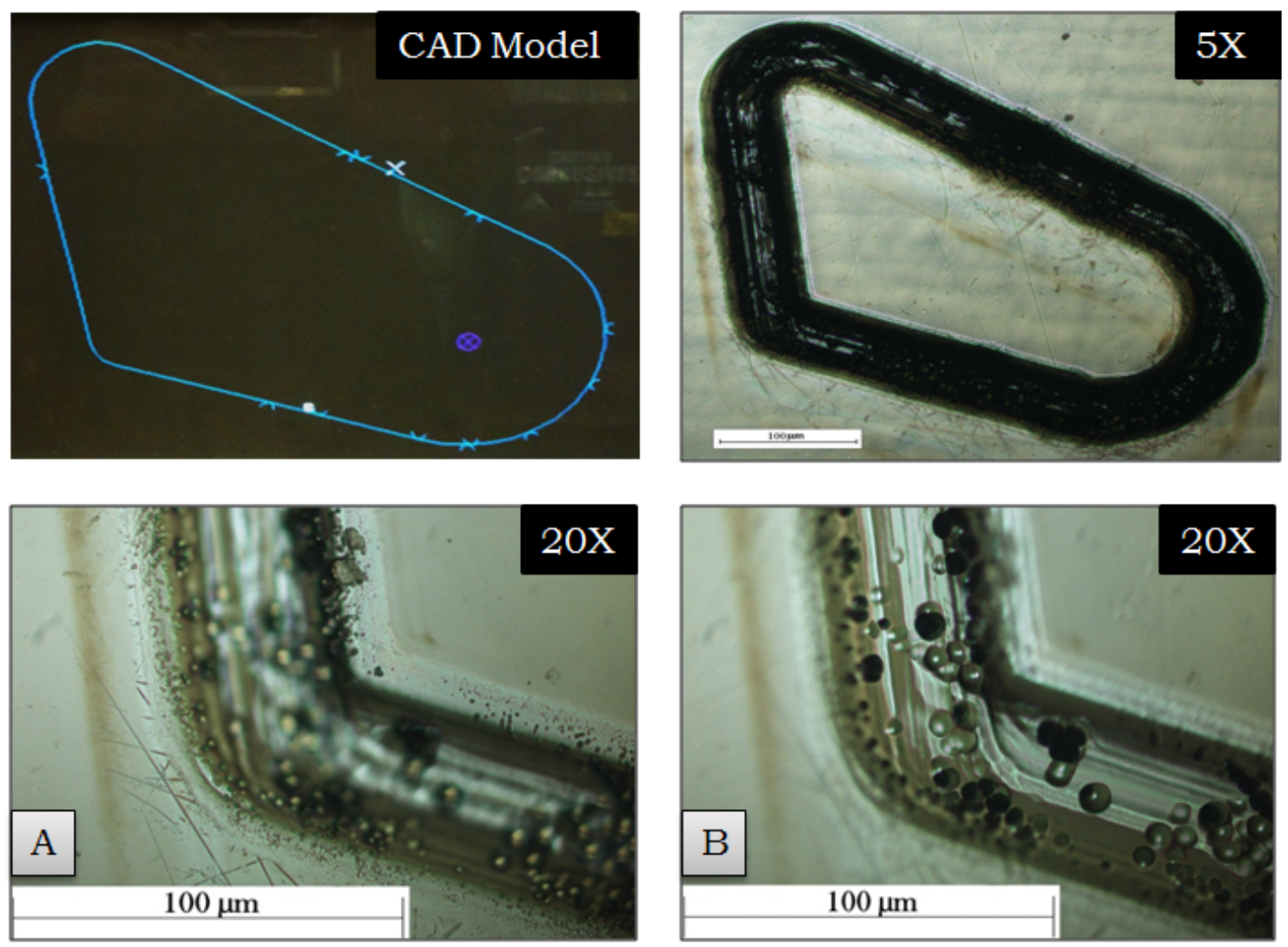

Figure 56 Electric microcircuit pattern made using CimCAD software, ablated pattern at $E=250 \mathrm{~mJ}, A-B)$ Optical microscope focus at surface of the seam, and at bottom of the seam 

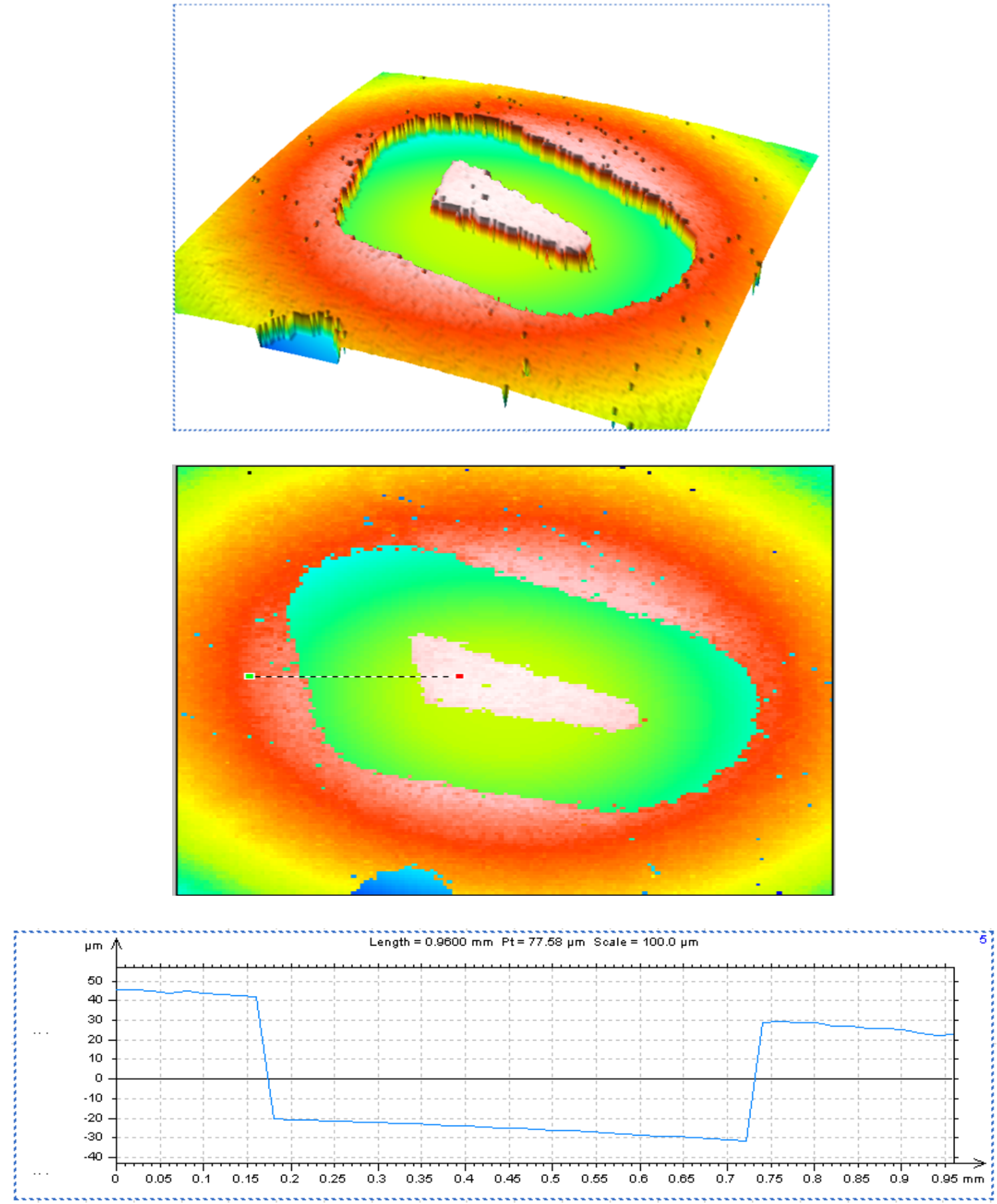

Figure 57 3D view, Top view of a sample pattern and line profile of an edge 
At the center of the seam, after ablation, melting is observed in the form of molten droplets of polyurethane which suggests ejection of decomposition products reunited or redeposited after ablation. Due to the nature of line micromachining, the formation of ripple like structure are observed on thin $(0.01 \mathrm{~mm})$ polymers samples. The shape of the ripples at the center of the seam clearly states the direction of the laser micromachining. The molten droplets of decomposition products exhibits two distinct types identified by their diameters; mainly small and large. Small droplets are observed around the edges of the seam having diameter of 4-10 $\mu \mathrm{m}$, whereas the large droplets consistently shown diameters in the range of $36-40 \mu \mathrm{m}$ are found at the center of the seam. Due to pulsed heating of the substrate the laser beam creates a uniform high temperature gradient along its path. This temperature gradient guides the molten debris from edges of the seam to the center, which could be the reason of larger diameter of droplets at the center as compared to the edges of the seam. The melting pool at the center of the seam could be due to ejected debris or decomposition products redeposited back onto the surface of the substrate. Therefore, most of the heat from ejected debris might go back into the substrate thus causing thermal damage to the surface as seen in the optical images. The formation of the molten droplets after ablation is also due to shorter step size distance and/or shorter feed rate along with high fluence. The further experiments in other similar polymer substrates (PET, PMMA) proved to be useful in elimination of molten droplets of matter. Thus, for every kind of polymer, a careful determination for suitable set of input parameters is required before achieving the best seam quality, optimum ablation depth and lower heat affected zones. 


\subsubsection{Ablation Mechanism in Etched Pattern}

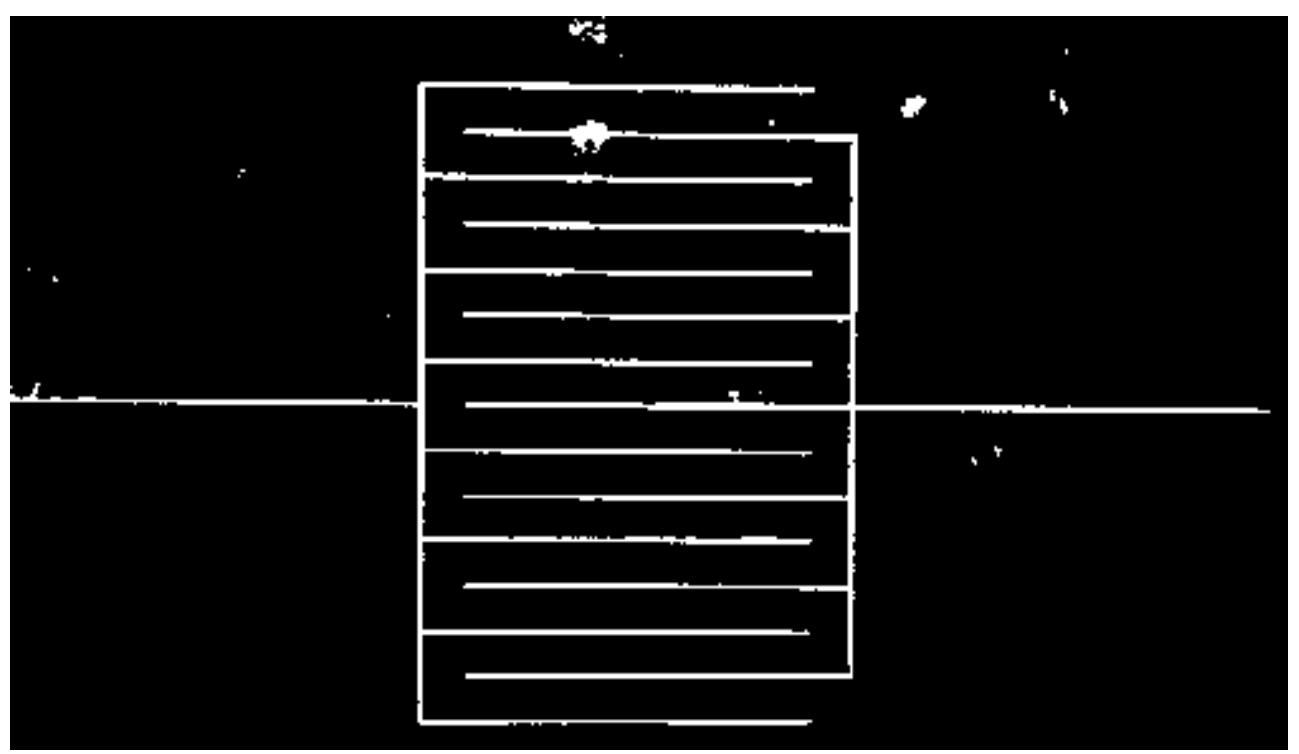

Figure 58 A sample electronic microcircuit pattern

In order to further investigate the nature of laser micromachining nature of polyurethane substrate, both in air and underwater micromachining was performed for a 2D sample electronic microcircuit pattern shown in Fig 58. In this experiment, two different types of mask sizes $(1152 \mu \mathrm{m}$ and $455 \mu \mathrm{m})$ are used to perform 2D micromachining for both in air and underwater on polyurethane (PU) sample. The along with the original pattern, the pattern is also scaled to $25 \%$ to observe changes in seam quality, heat affected zone, ablation depth, difference in underwater and in air micromachining. From previous experimental observations, the best input parameters are determined and used for both experiments as $\mathrm{E}=250 \mathrm{~mJ}, \mathrm{~Hz}=25 \mathrm{~Hz}$, feed rate $=1$ $\mu \mathrm{m} /$ pulse. For underwater micromachining the contained is filled with $20 \mathrm{ml}$ water and polymer sample was fully submerged under water. The laser beam diameter is observed 
to be $76.5 \mu \mathrm{m}$, the laser irradiated area rise up and the intended pattern of electronic microcircuit was obtained with a uniform ablation depth of $\sim 80 \mu \mathrm{m}$.

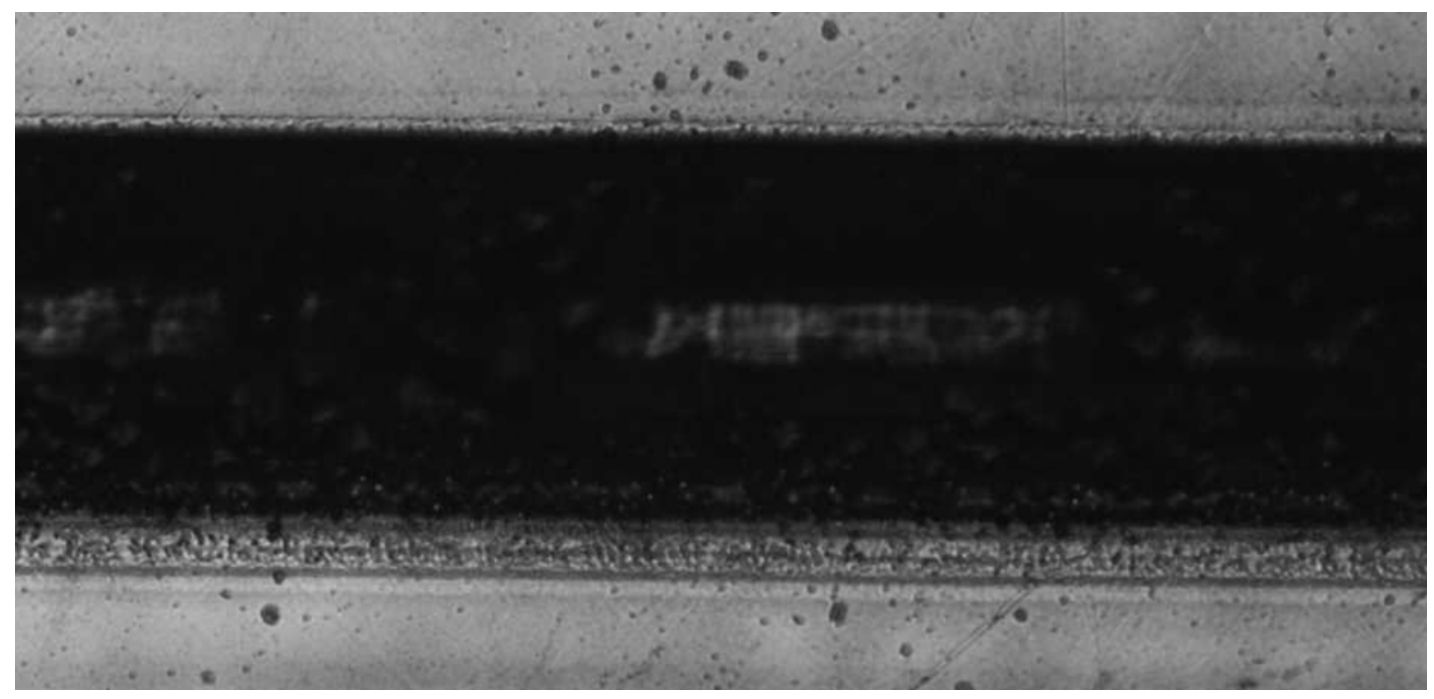

Figure 59 At 20x, Seam quality observed under "in air" environment for polyurethane substrate

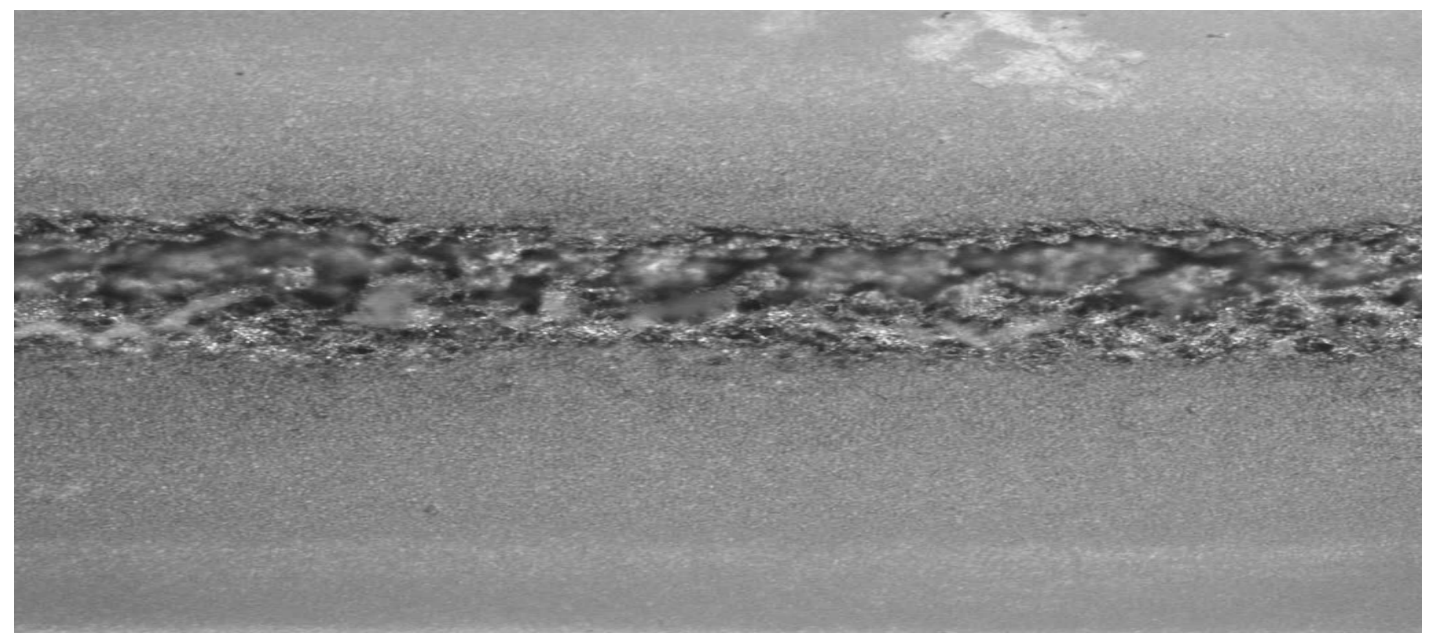

Figure 60 At 20x, Seam quality submerged “under water" environment for polyurethane substrate 
From Figs. 59-60, the 2D pattern micromachining phenomenon for both air and underwater was found out to be distinctively different from each other. The sample micro-machined using laser in air demonstrated sharp edges as compared to the specimen micro-machined underwater with same set of input parameters. High seam quality with very low heat affected zone is observed when performing micro-machining in air as compared to underwater. For in air environment, no debris is observed along edges of the seam, however for underwater micromachining the ablation bi-products are observed to be entrapped inside the seam. A uniform ablation depth of $\sim 80 \mu \mathrm{m}$ is observed throughout the laser irradiated area for 'in air' substrate, however for underwater substrate the ablation depth is less than $\sim 5 \mu \mathrm{m}$. It suggests that the input energy is not enough to break down bonding for under water substrate therefore the above figure for underwater suggests incubation effect. Also for underwater specimen, no signs of molten pool at the center are observed due to cooling effect of the water which rapidly cools down any debris or ejected decomposition products after ablation.
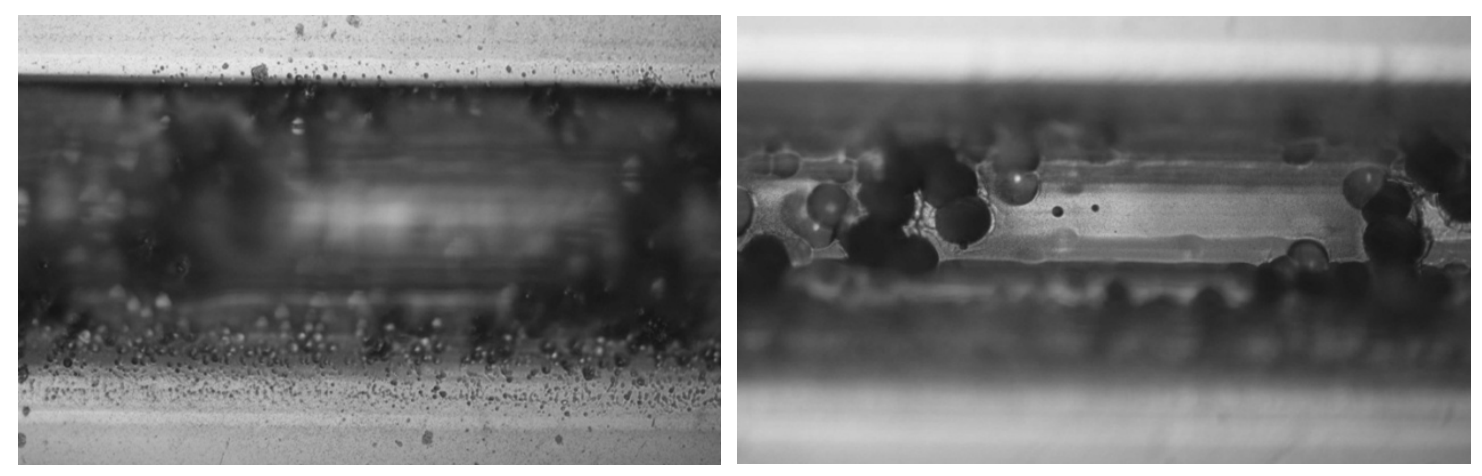

Figure 61 For in air ablation a) Focus at top surface of the seam b) Focus at bottom surface of the seam 
In Fig. 61, the ablation mechanism of the in air specimen provide evidence of a molten pool at the bottom of the seam clearly visible in variety of small to large diameter droplets. This imply that the fluence is not enough to completely ablate the polymer but powerful enough to create a molten pool of droplets; which implies photo-thermal mode of ablation. The numbers of molten droplets at the center of the beam are found to be much lesser as compared to the droplets on the edges of the seam boundary. This difference is observed due to the variation in the diameter of the droplets at the center as compared to the edges. This also implies that smaller droplets at the edges converge at the center to form the larger diameter droplets of molten decomposition bi-products as a result of the ablation. Sharp edges and corners are observed throughout the etched pattern. 


\subsection{Micromachining of a Gear Pattern}

\subsubsection{Scaling Effect of Mask Diameter}

A sample gear pattern shown in Fig. 62 was ablated on two different materials, namely, polyurethane $(1 \mathrm{~mm}$ thick $)$ and PET polymer sheet $(0.1 \mathrm{~mm}$ thick $)$ in air. The results of these experiments are given in the following sections.

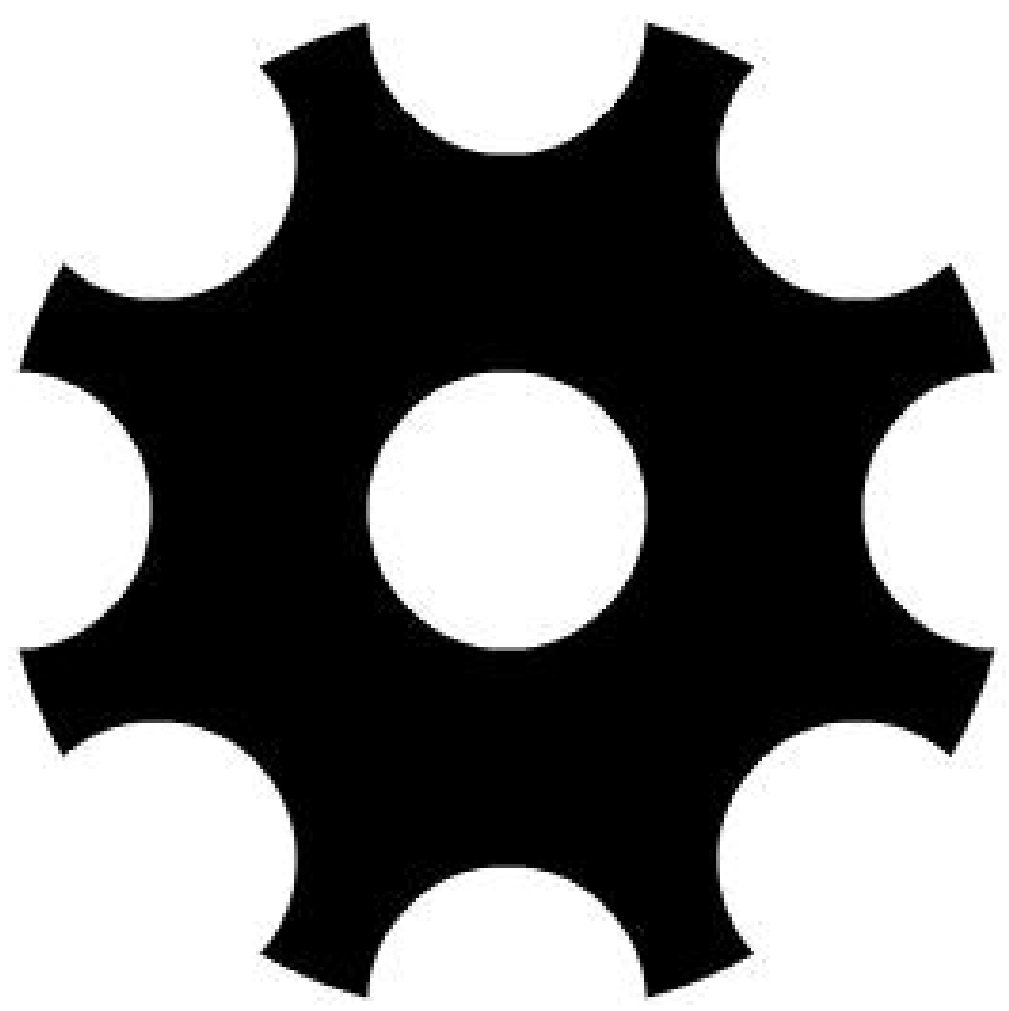

Figure 62 A sample gear pattern of having an outer diameter of $2.4 \mathrm{~mm}$ 
Table 9 Experiments for a sample gear pattern

\begin{tabular}{|c|c|c|c|c|c|c|c|}
\hline $\begin{array}{c}\text { Test } \\
\text { No. }\end{array}$ & $\begin{array}{c}\text { Energy } \\
\left(\mathbf{m} \mathbf{J} / \mathbf{c m}^{2}\right)\end{array}$ & $\begin{array}{c}\text { Repetition } \\
\text { Rate }(\mathbf{H z})\end{array}$ & $\begin{array}{c}\text { Sample } \\
\text { Material }\end{array}$ & $\begin{array}{c}\text { Thickness } \\
(\mathbf{m m})\end{array}$ & $\begin{array}{c}\text { Mask } \\
\text { Dia. } \\
(\boldsymbol{\mu m})\end{array}$ & Scale & $\begin{array}{c}\text { Final } \\
\text { Pattern } \\
\text { Diameter } \\
(\mathbf{m m})\end{array}$ \\
\hline 1 & 250 & 20 & Polyurethane & 1 & 1152 & $100 \%$ & $\mathbf{2 . 4 m m}$ \\
\hline 2 & 250 & 20 & Polyurethane & 1 & 555 & $50 \%$ & $\mathbf{1 . 2 m m}$ \\
\hline 3 & 250 & 20 & PET & 0.1 & 1152 & $100 \%$ & $\mathbf{2 . 4 m m}$ \\
\hline 4 & 250 & 20 & PET & 0.1 & 555 & $50 \%$ & $\mathbf{1 . 2 m m}$ \\
\hline 5 & 250 & 20 & PET & 0.1 & 300 & $25 \%$ & $\mathbf{0 . 6 m m}$ \\
\hline 6 & 250 & 20 & PET & 0.1 & 180 & $15 \%$ & $\mathbf{0 . 3 6 m m}$ \\
\hline
\end{tabular}

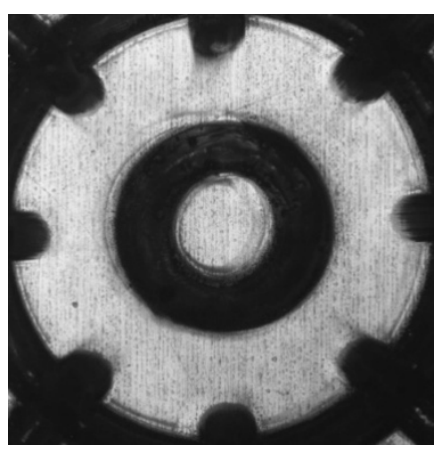

Test 1

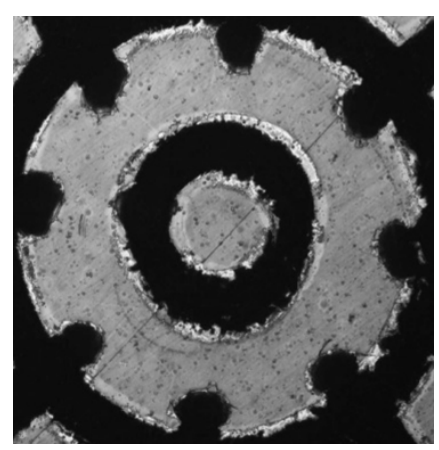

Test 4

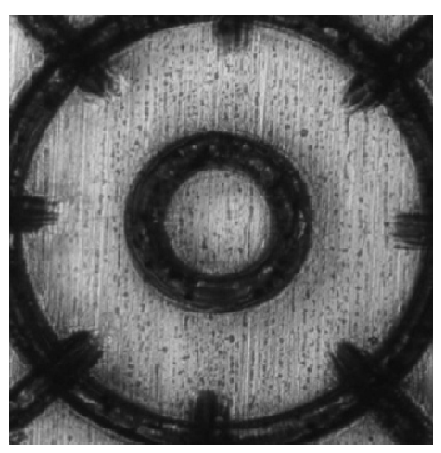

Test 2

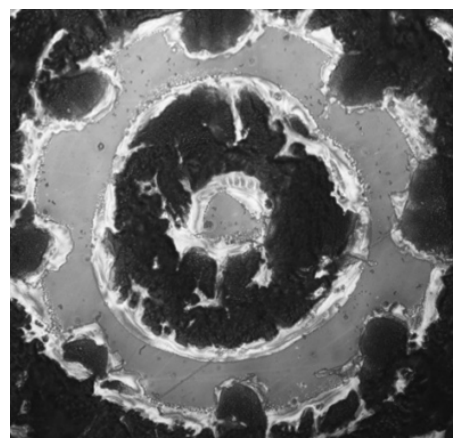

Test 5

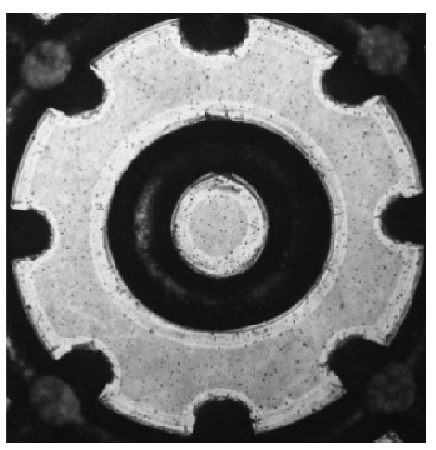

Test 3

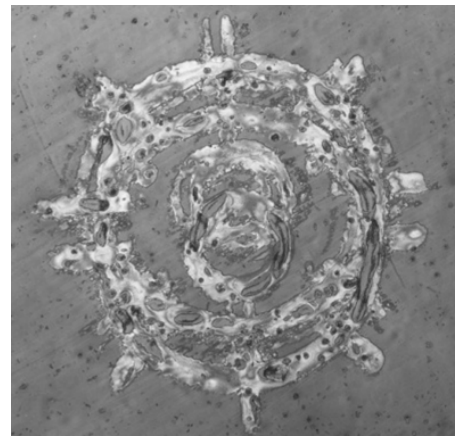

Test 6

Figure 63 Different samples etched with scale variation and material for the design of the gear pattern after laser etching 


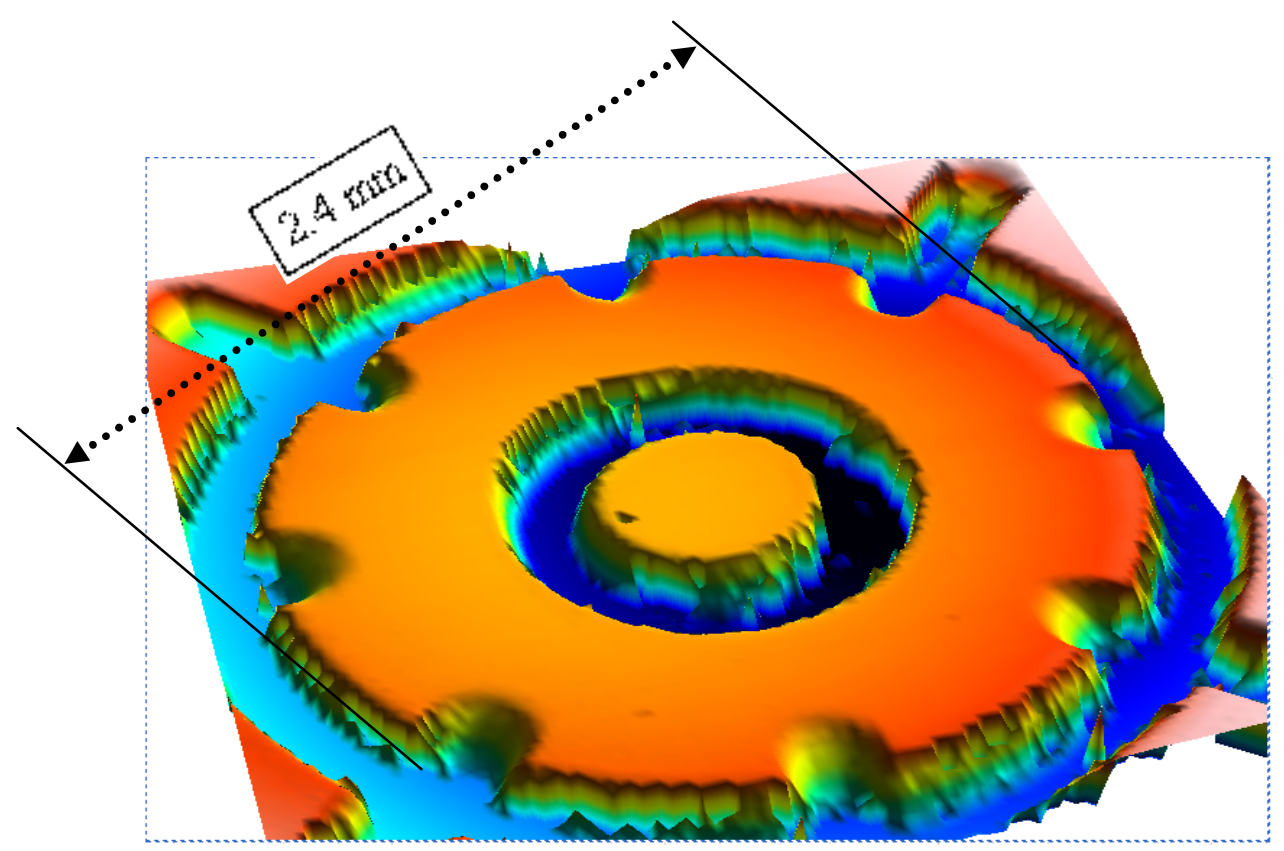

Figure 64 For test 1; 3D surface profile for a gear etched with mask size of $1152 \mu \mathrm{m}$ on polyurethane sample ( $1 \mathrm{~mm}$ thickness) having $2.4 \mathrm{~mm}$ diameter

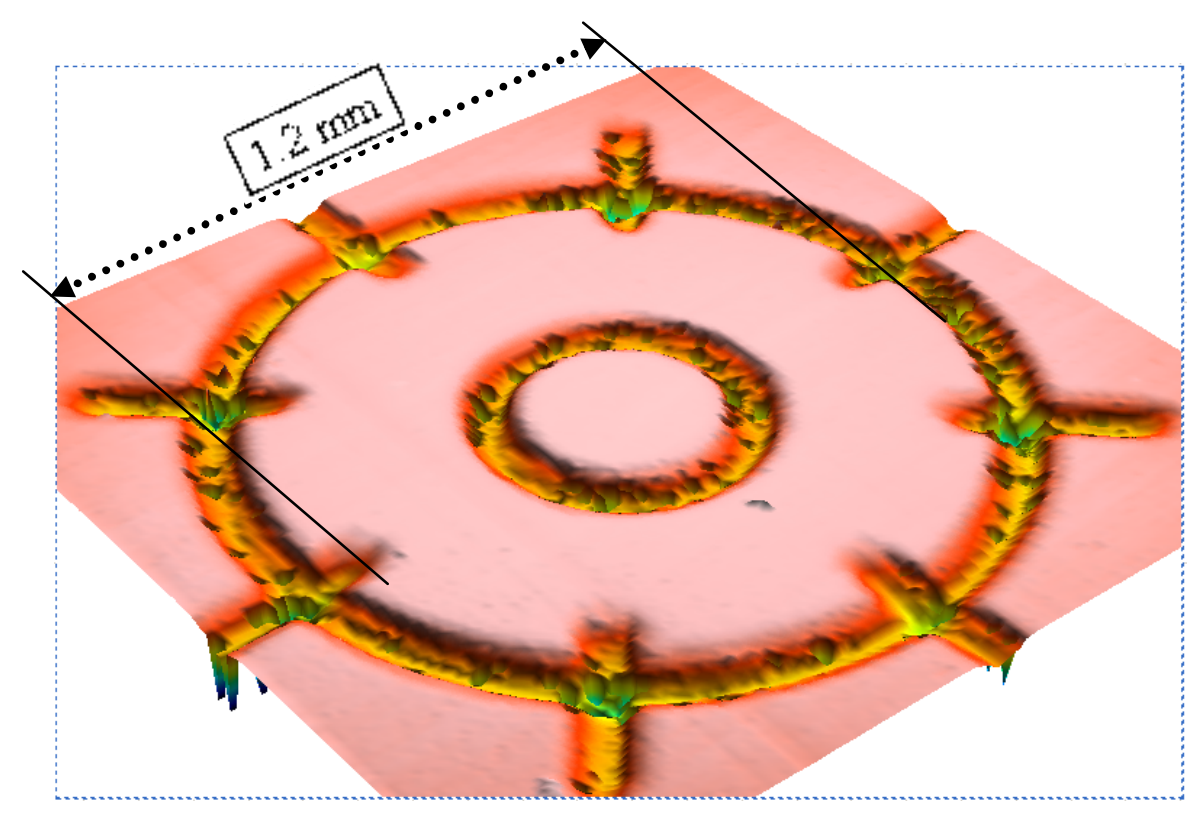

Figure 65 For test 2; 3D surface profile of a gear etched with mask size of $555 \mu \mathrm{m}$ on polyurethane sample (1mm thickness) having $1.2 \mathrm{~mm}$ diameter 


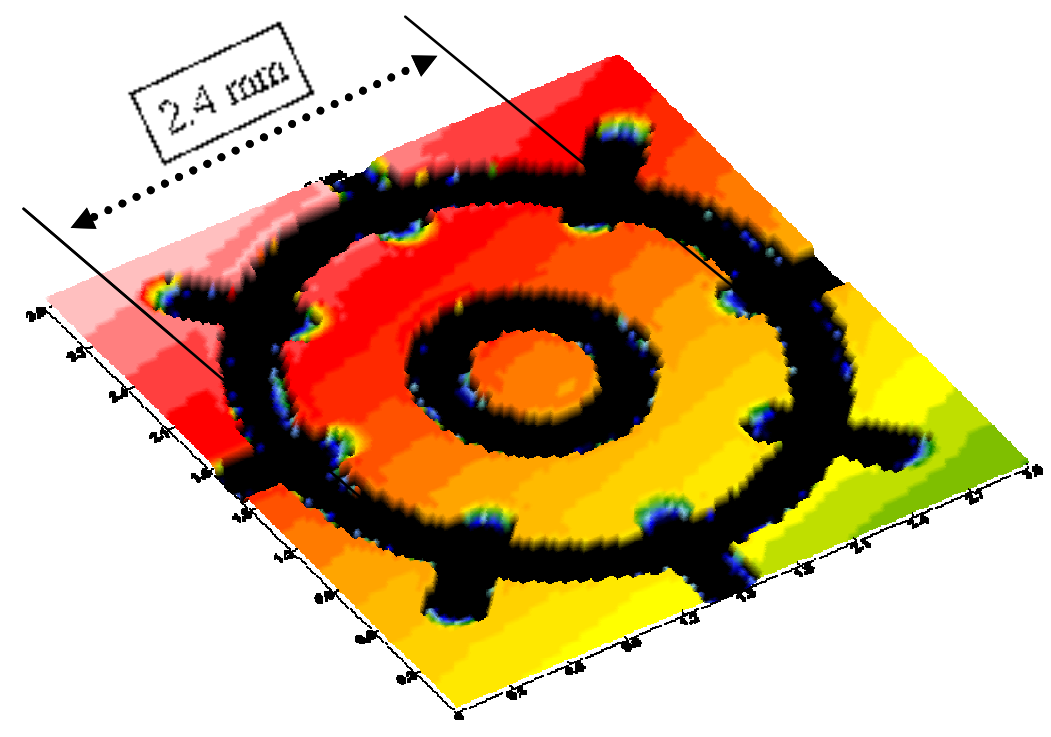

Figure 66 for test 3; 3D surface profile for a gear etched with mask size of $1152 \mu \mathrm{m}$ on PET sample (0.1mm thickness) having $2.4 \mathrm{~mm}$ diameter

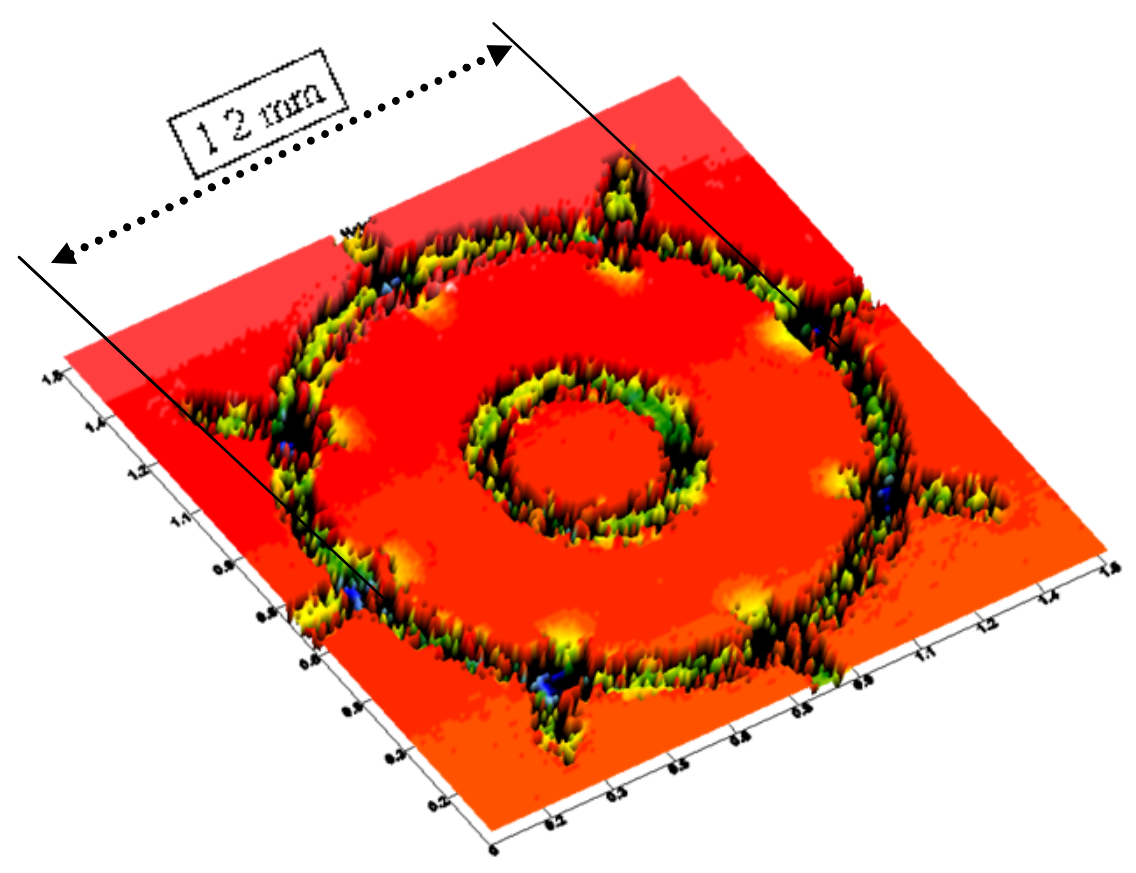

Figure 67 For test 4; 3D surface profile for a gear etched with mask size of 555 $\mu \mathrm{m}$ on PET polymer sample $(0.1 \mathrm{~mm}$ thickness) having $1.2 \mathrm{~mm}$ diameter 


\subsubsection{Results and Discussion}

The time required for each pattern to be created varied upon the repetition rates, lead, tail and step size distance entered into the program. The total time for each pattern is observed to be decreasing proportionally to its intended scale and/or diameter value required.

For test 1 , using $1152 \mu \mathrm{m}$ mask diameter the maximum ablation depth is observed to be $\sim 100 \mu \mathrm{m}$ under air environment with final gear pattern outer diameter obtained as 2.4 mm. Sharpe edges of gear teeth were also observed. The specimen material used is polyurethane sheet of $10 \times 10 \mathrm{~mm}$ dimensions with $1 \mathrm{~mm}$ thickness.

For test 2, using $555 \mu \mathrm{m}$ mask diameter the maximum ablation depth is observed to be $55 \mu \mathrm{m}$ under air environment with final gear pattern scaled at $50 \%$ to its original size. The final diameter of the gear pattern is observed to be $1.2 \mathrm{~mm}$. Sharpness of gear teeth is decreased due to scaling and unchanged input energy and repletion rates. The specimen material used is polyurethane sheet of $10 \times 10 \mathrm{~mm}$ with $1 \mathrm{~mm}$ thickness.

For test 3 , using the mask diameter of $1152 \mu \mathrm{m}$ the maximum ablation depth of $\sim 85 \mu \mathrm{m}$ is observed under air excimer laser micromachining process. Small cracks alongside the edges of the gear teeth are observed due to high repetition rates used during shortest feed rate. The final diameter of the gear pattern is observed to be $2.4 \mathrm{~mm}$. The specimen material used is PET polymer sheets of $10 \times 10 \mathrm{~mm}$ area at $0.1 \mathrm{~mm}$ thickness. 
For test 4 , the mask diameter of $555 \mu \mathrm{m}$ is used to obtain the final etched gear pattern diameter of $1.2 \mathrm{~mm}$ with maximum ablation depth of $30 \mu \mathrm{m}$ under air environment. Sharpe edges are not observed along with the edges; the edge quality is very poor as compared to test 3 . A scaling factor of $50 \%$ is used on $10 \times 10 \mathrm{~mm}$ PET polymer sheet of $0.1 \mathrm{~mm}$ thickness.

For test 5 , the mask diameter of $300 \mu \mathrm{m}$ is used to obtain the final etched gear pattern diameter of $0.6 \mathrm{~mm}$ with maximum ablation depth of $10 \mu \mathrm{m}$ under air environment. The edges of the pattern showed debris all around the gear pattern with distortion and no sharp edges. A scaling factor of $25 \%$ is used on $10 \times 10 \mathrm{~mm}$ PET polymer sheet of 0.1 mm thickness.

For test 6 , the mask size of $180 \mu \mathrm{m}$ is used to obtain the final etched gear pattern diameter of $0.36 \mathrm{~mm}$ with no ablation depth. The mask diameter and input energy are not able to ablate any significant amount of material but managed to scribe the surface. A scaling factor of $15 \%$ is used on $10 \times 10 \mathrm{~mm}$ PET polymer sheet of $0.1 \mathrm{~mm}$ thickness. 


\subsubsection{Effect of Mask Diameter on Seam Quality}

The Figs. 68 to 71 shows 3D surface mapping scans highlighting critical features about seam quality for recently etched gear pattern
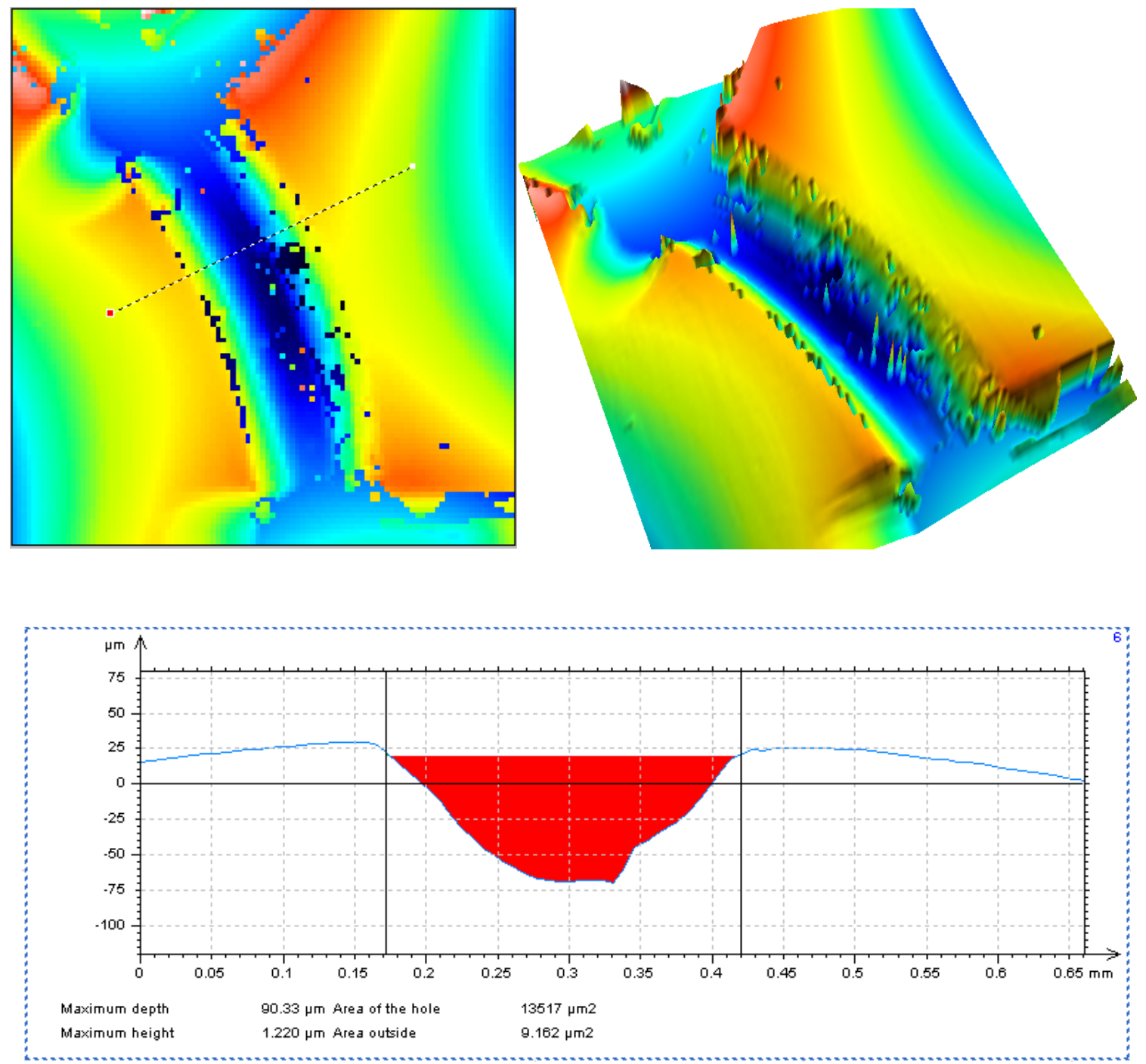

Figure 68 For test 1; 3D surface profile for a gear etched along with line profile at running seam for polyurethane sample (1 $\mathrm{mm}$ thickness) etched gear $(2.4 \mathrm{~mm}$ dia.) with mask size of $1152 \mu \mathrm{m}$ 

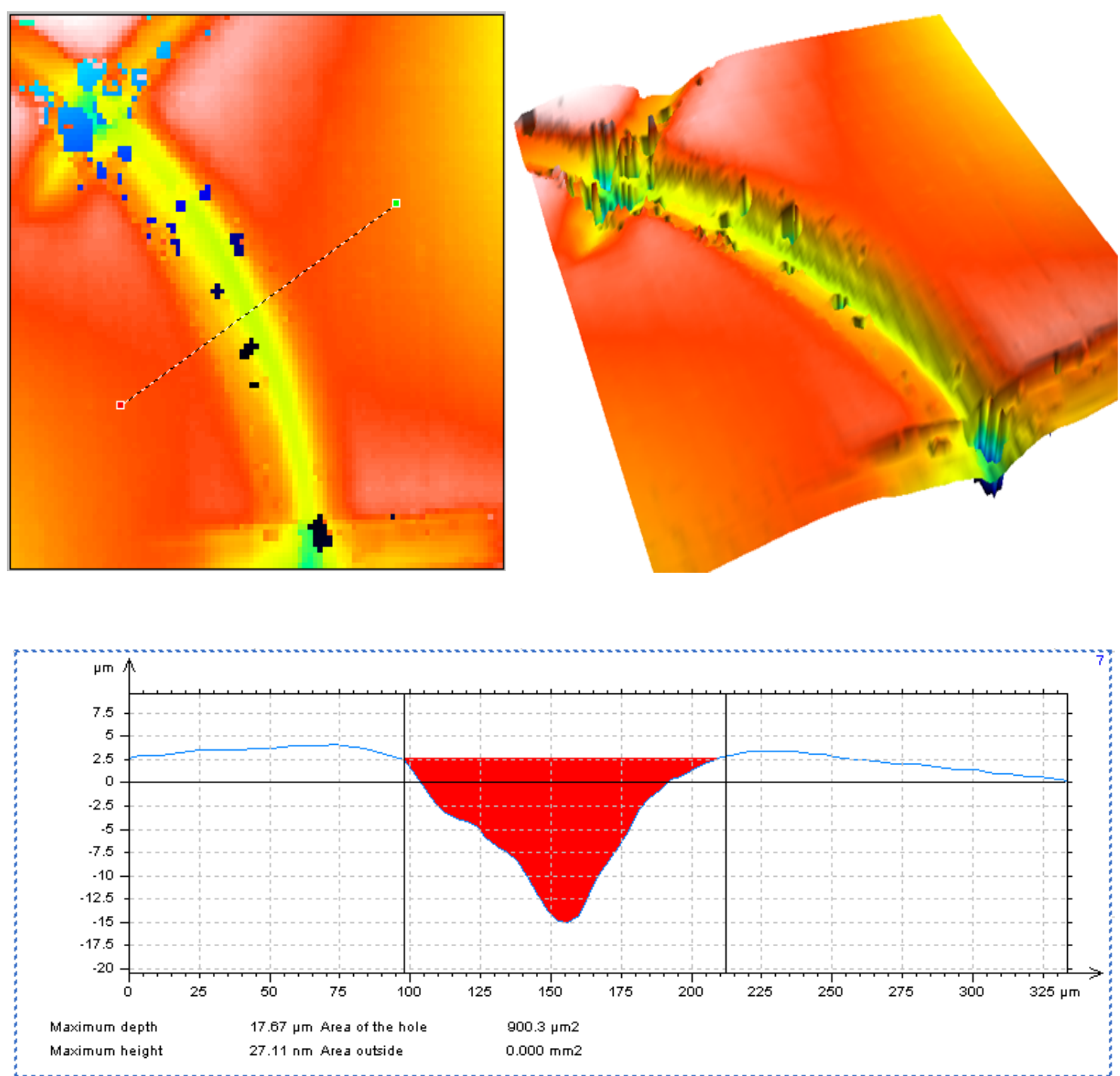

Figure 69 For test 2; 3D surface profile for a gear etched along with line profile at running seam for polyurethane sample $(1 \mathrm{~mm}$ thickness $)$ etched gear $(1.2 \mathrm{~mm}$ dia.) with mask size of $555 \mu \mathrm{m}$ 

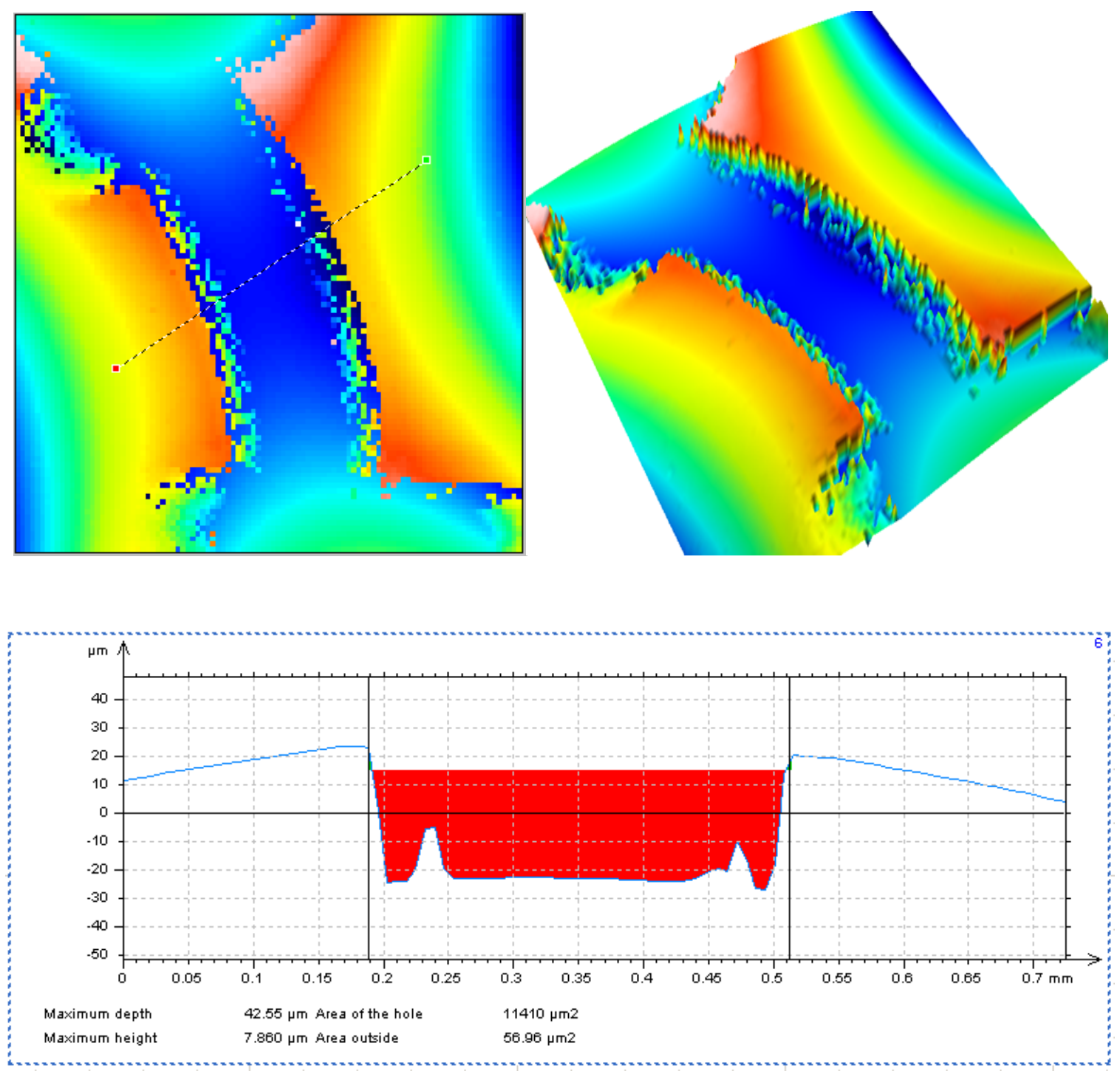

Figure 70 For test 3; 3D surface profile for a gear etched along with line profile at running seam for PET polymer sample (0.1 $\mathrm{mm}$ thickness) etched gear( $2.4 \mathrm{~mm}$ dia.) with mask size of $1152 \mu \mathrm{m}$ 

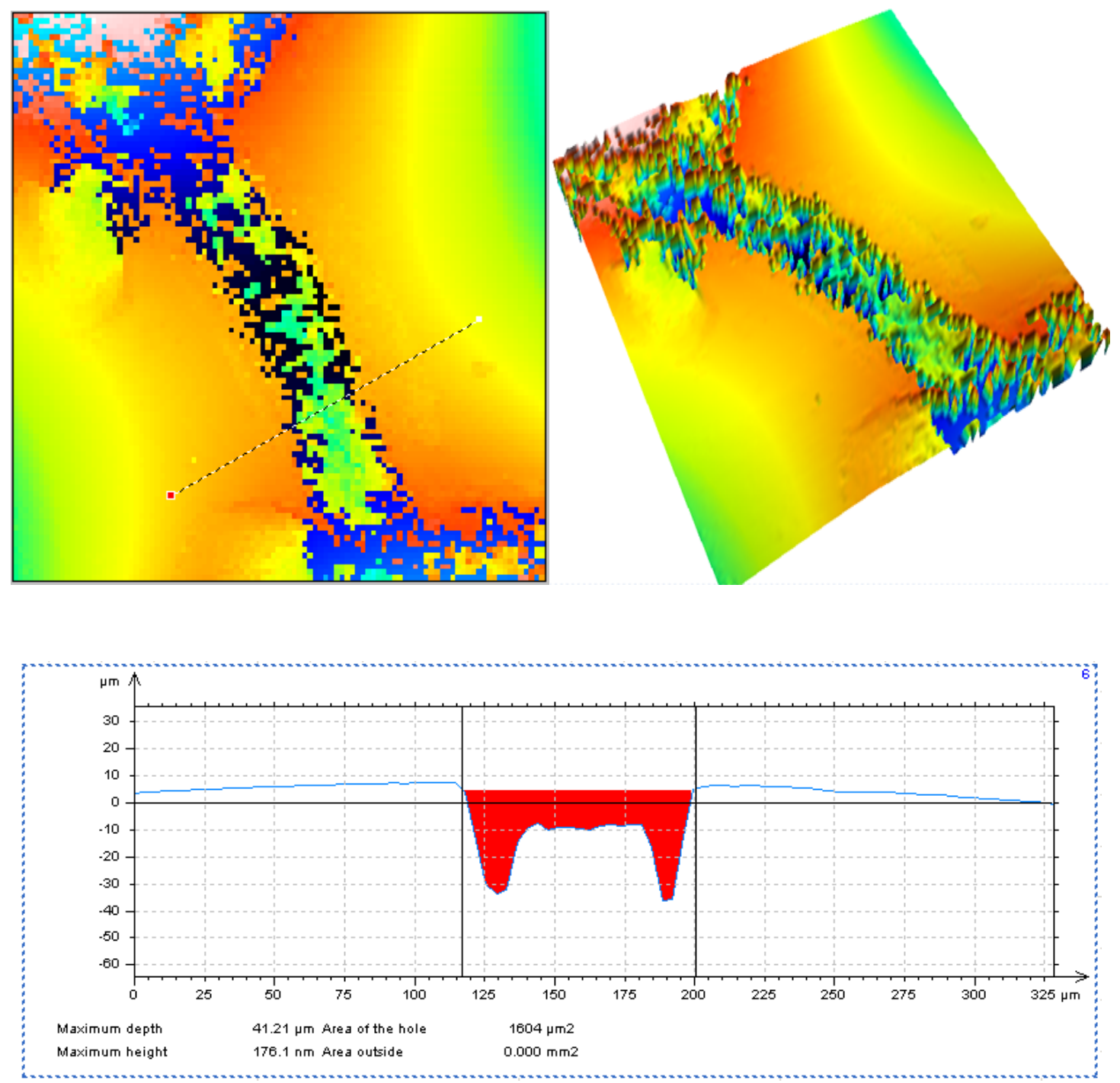

Figure 71 For test 4; 3D surface profile for a gear etched along with line profile at running seam for PET polymer sample (0.1 mm thickness) etched gear(1.2 $\mathrm{mm}$ dia.) with mask size of $555 \mu \mathrm{m}$ 
The Fig. 72 to 77 shows optical images highlighting critical features about seam quality for recently etched gear pattern:
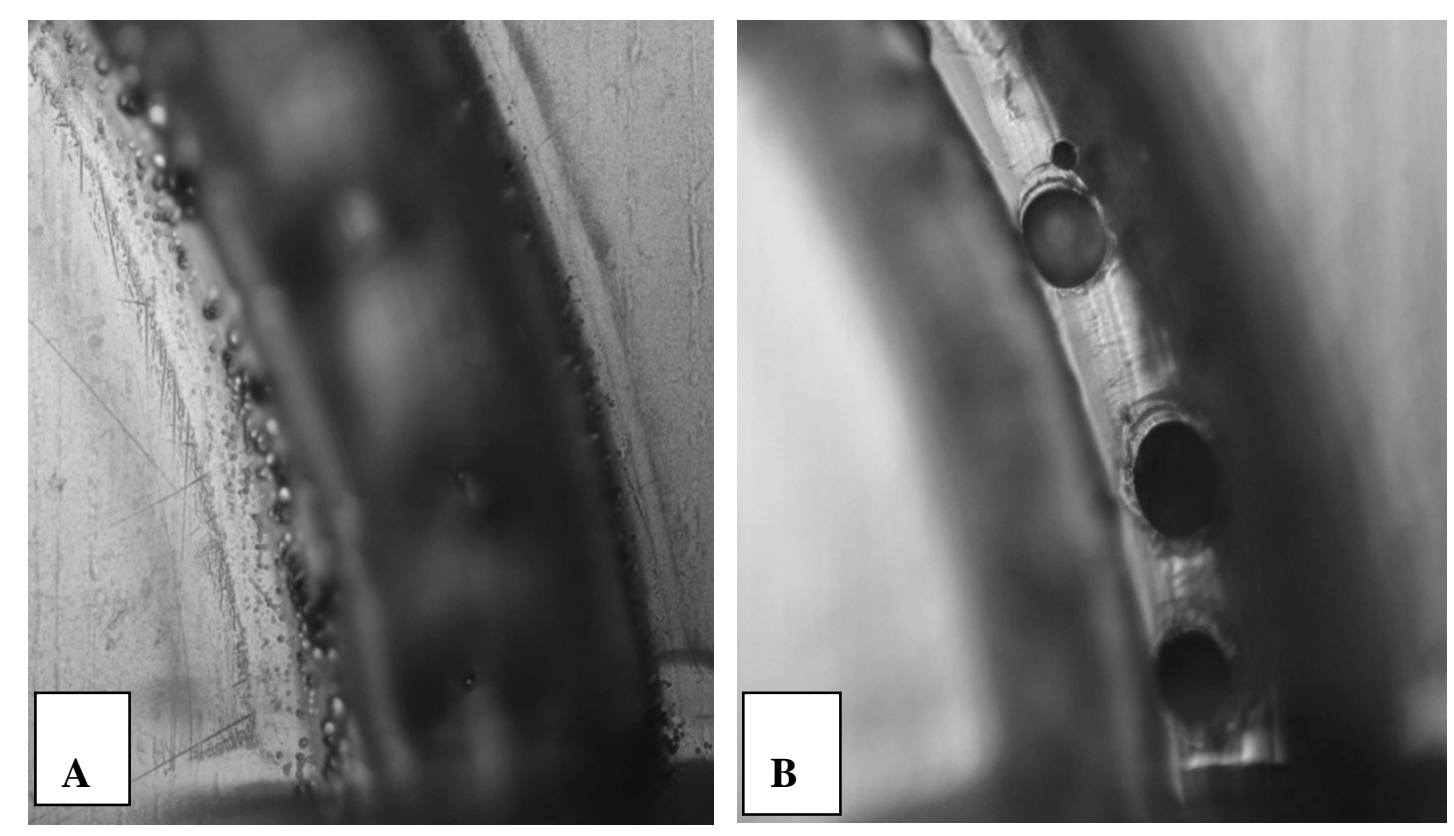

Figure 72 For test no. 1, Seam/Edge quality of gear pattern a) Focus at top surface

b) Focus at bottom surface 

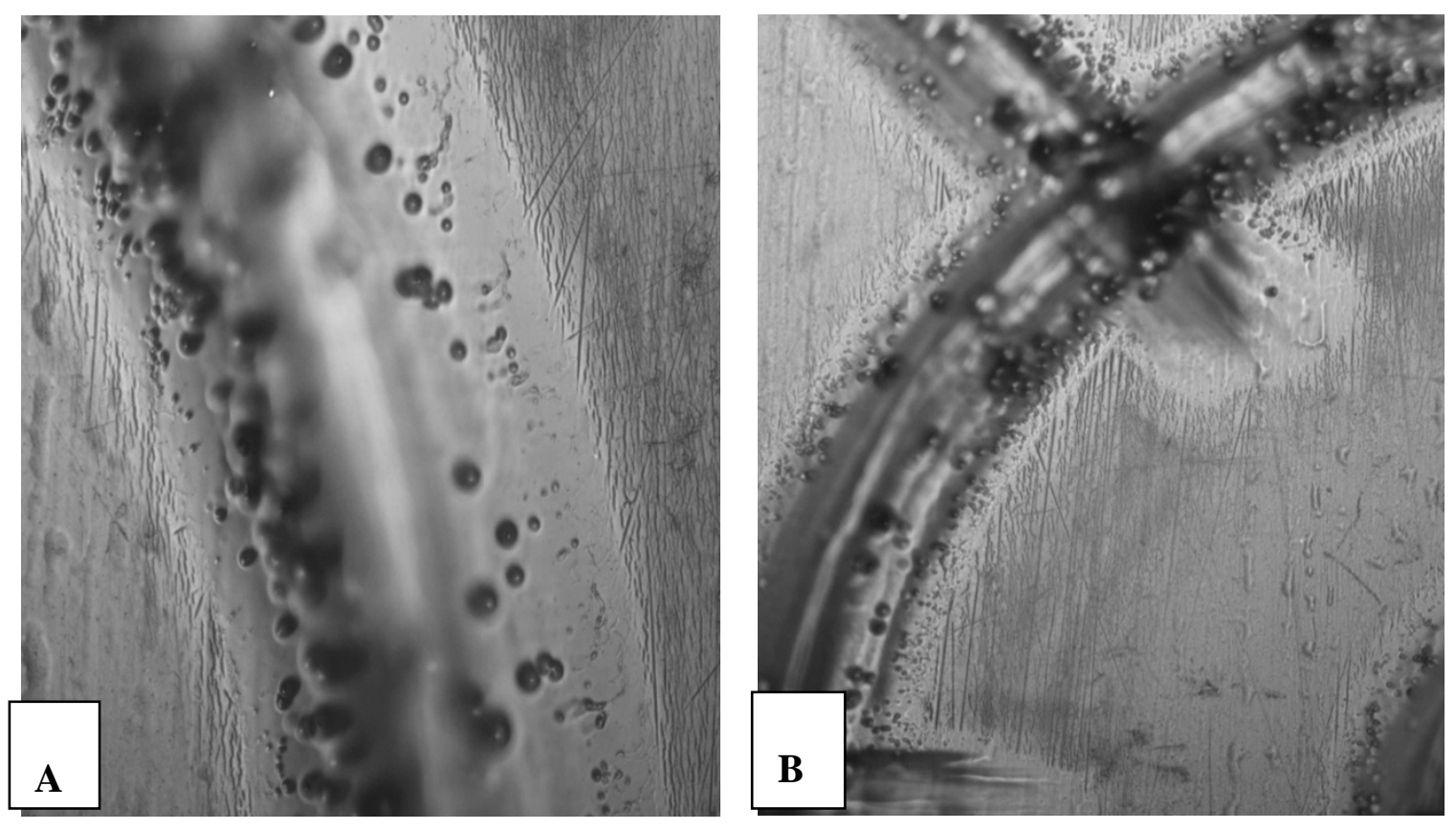

Figure 73 For test no. 2, Seam/Edge quality of gear pattern
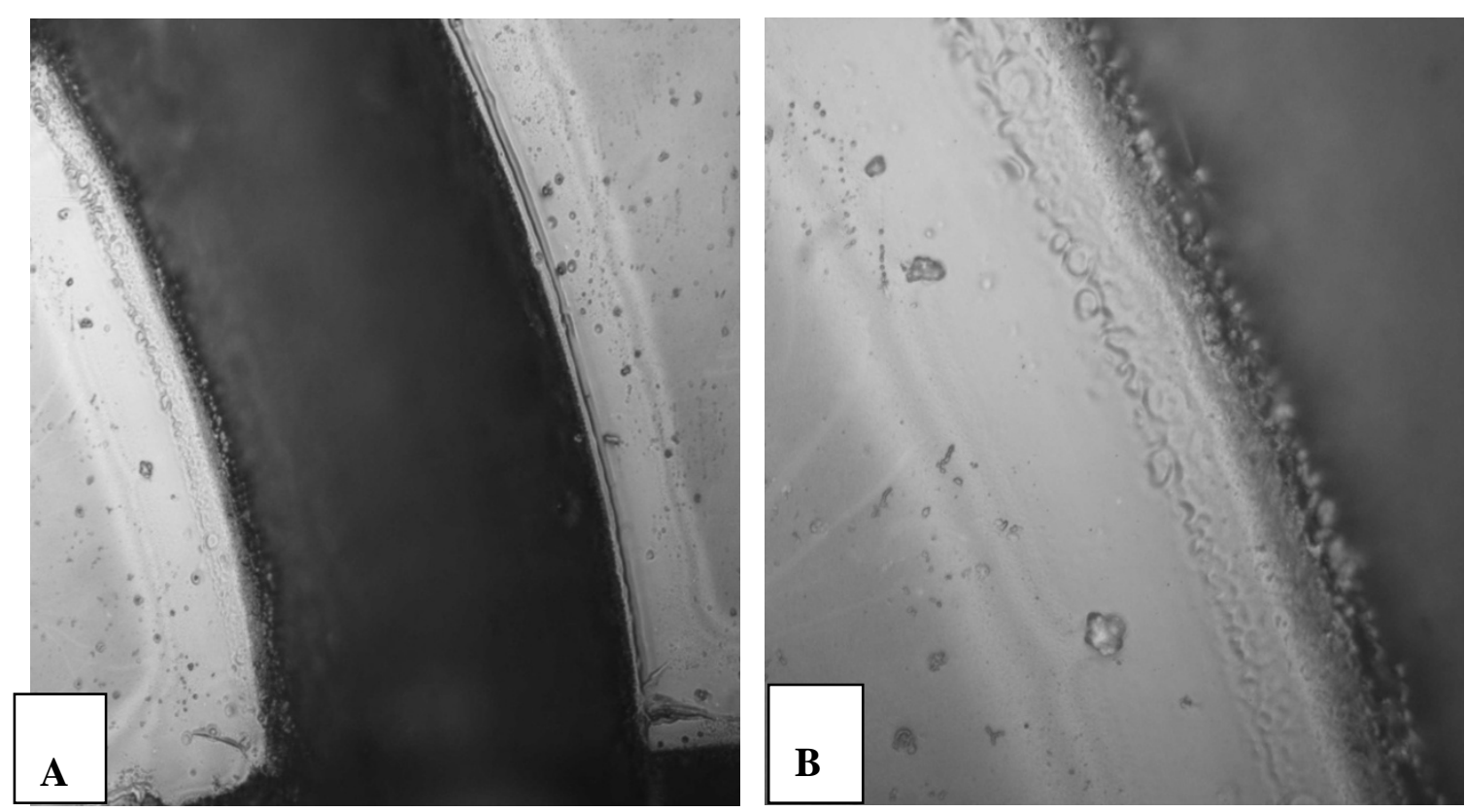

Figure 74 For test no. 3, Seam/Edge quality of gear pattern a) At 20x focus on top surface b) At 50x 

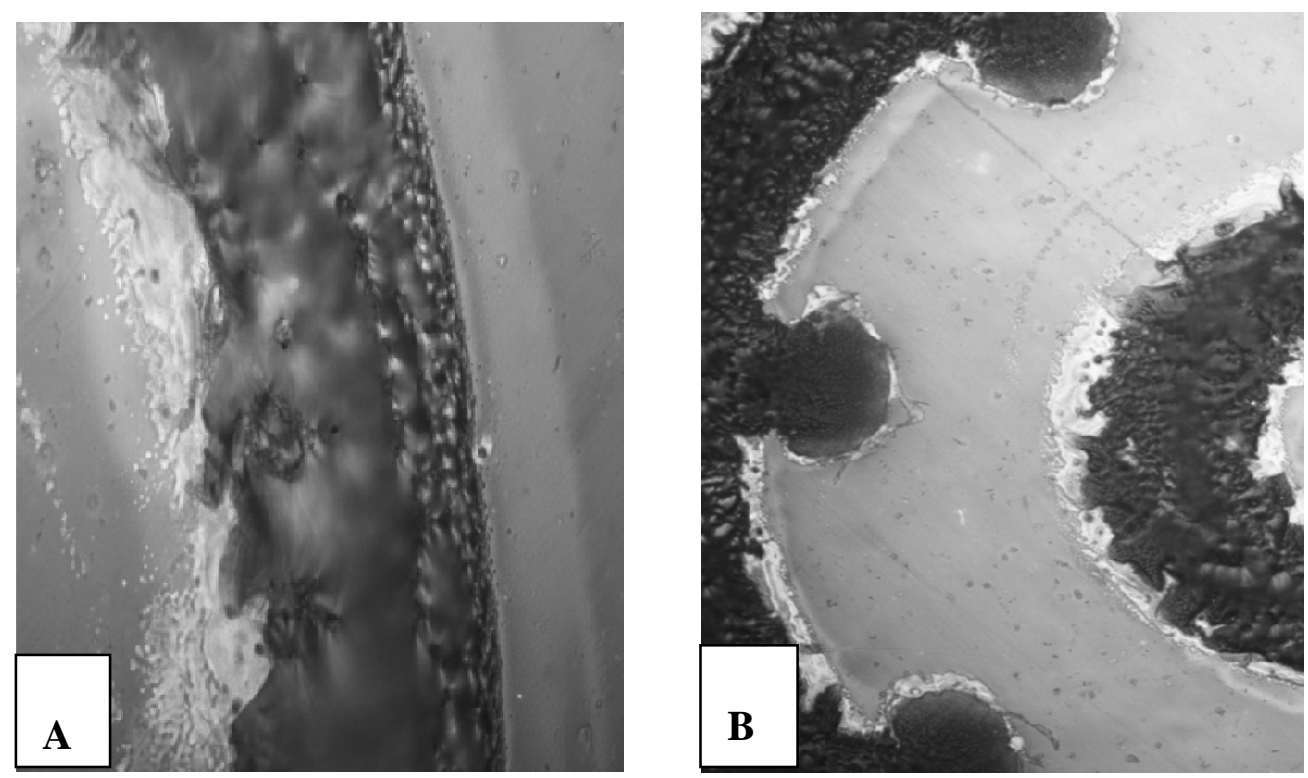

Figure 75 For test no. 4, Seam/Edge quality of gear pattern a) At 50x focus on top surface b) At 20x
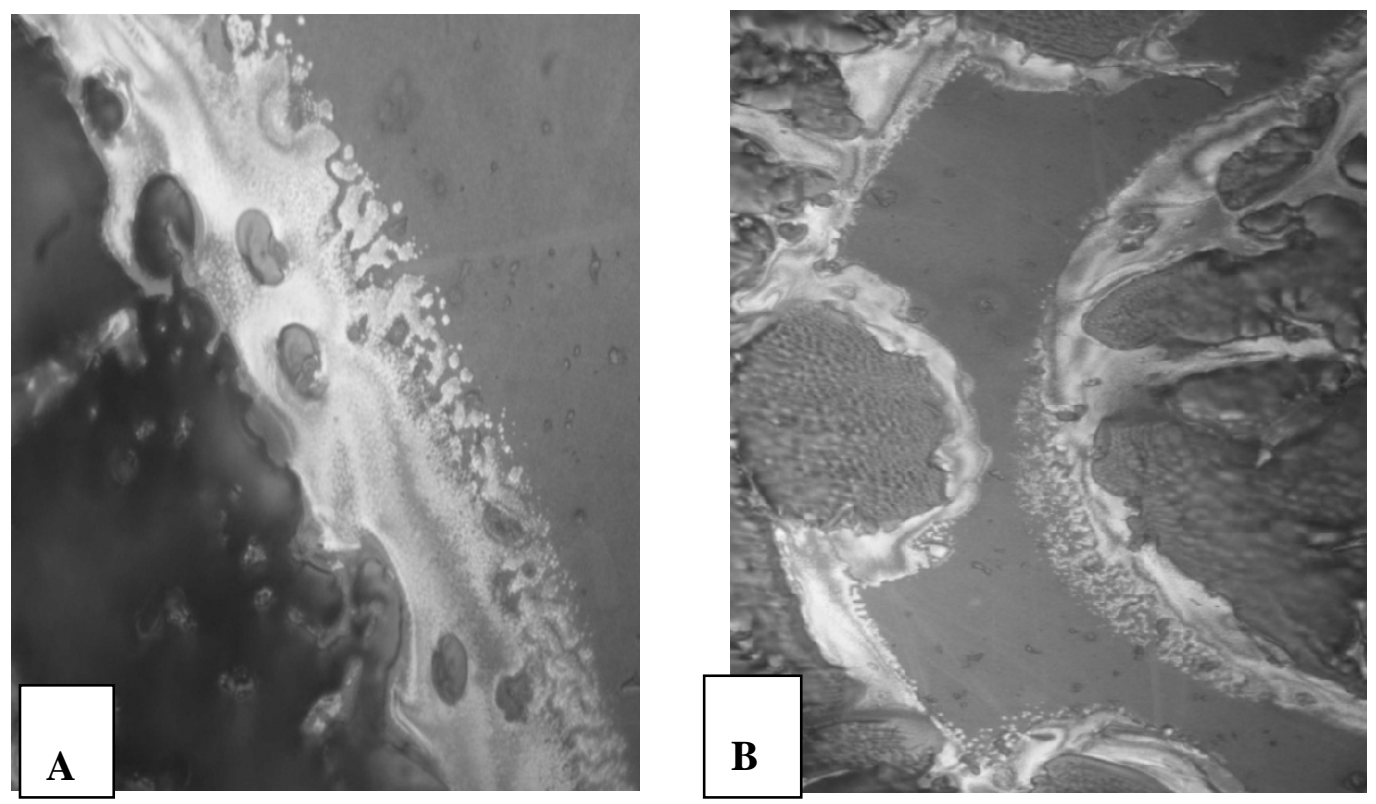

Figure 76 For test no. 5, Seam/Edge quality of gear pattern a) At 100x focus on top surface b) At 50x 

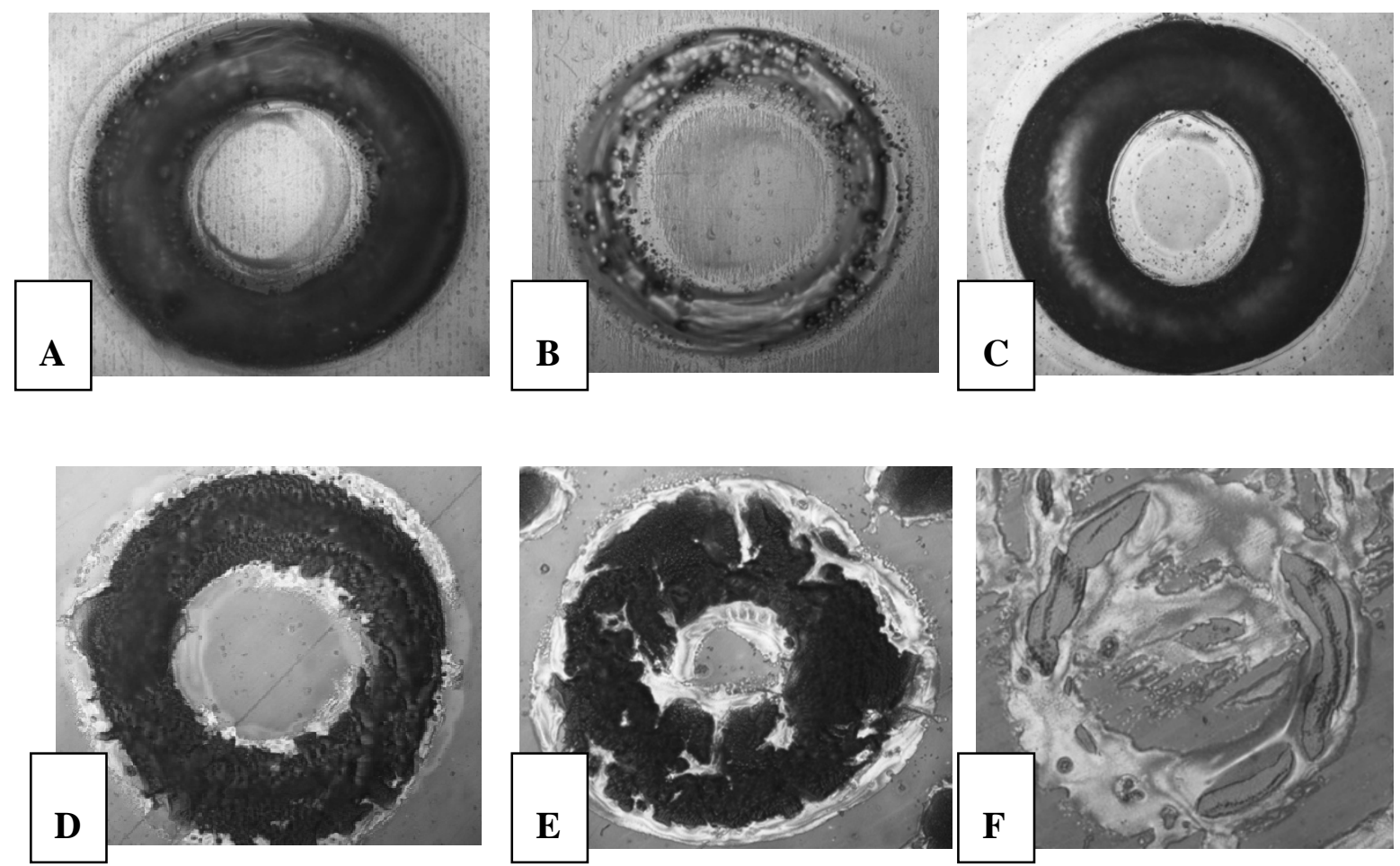

Figure 77 For test no. 1-6, at 20x Seam/Edge quality at the center of a) Test 1, b) Test 2, c)

Test 3, d) Test 4, e) Test 5, f) Test 6

\subsubsection{Results and Discussion}

For test 1 , the seam quality is observed to be high with uniform heat affected zone throughout the etched pattern as shown in Fig. 72(a). The resulting surface topography from this test is consistent with previous samples of polyurethane used for pattern etching using excimer laser. In Fig. 72(b), the optical focus is shifted to the bottom surface of the seam which is located at the depth of $120 \mu \mathrm{m}$ from surface. Similar to the previous observations, the large bubble shaped droplets of molten decomposition products are observed at the center of the seam. The size of the molten droplets decreased as moved from center to the closest edge/arc of the seam. 
For test 2, in Fig. 73 the seam quality is observed to be relatively poor as compared to the test 1 where $1152 \mu \mathrm{m}$ mask diameter is used. The reason for this change in seam quality is due to smaller mask diameter used with same fluence as test 1 . The maximum ablation depth is also affected due to change in mask diameter. The width of the seam is observed to decrease by decreasing the mask diameter. The ablation phenomenon in this polyurethane specimen under air is consistent with test 1 where small droplets are observed at the edge but the size of molten droplets is increased by moving away from center and towards the edges.

For test 3, in Fig. 74, the thickness of specimen (polystyrene sheets) is changed to $0.1 \mathrm{~mm}$ which showed very high quality of seam with clearly visible heat affected zone along the seam. Sharp edges are observed in this pattern with less material buildup on the edges. In this case the ablation phenomenon is different from the polyurethane specimen as no molten decomposition products were observed in the shape of small molten droplets around the edges or center of the seam.

For test 4, the (PET polymer) sample with $0.1 \mathrm{~mm}$ thickness showed poor edge/seam quality as compared to test 3 due to smaller mask diameter of $555 \mu \mathrm{m}$ is used without any change in fluence. The final gear pattern diameter of $1.2 \mathrm{~mm}$ is observed with medium amount of debris splashed around the edges of the seam. With decreasing the mask diameter the heat affected zone thickness also uniformly decreases with significant decrease in ablation depth. 
For test 5, the polymer (PET polymer) sample with $0.1 \mathrm{~mm}$ thickness showed poor seam quality due to greater reduction in mask diameter to $300 \mu \mathrm{m}$. The signs of splashed debris are observed around the edges of the seam and local melting of the edges is observed. The final diameter of the sample pattern is observed to be $0.6 \mathrm{~mm}$ with maximum ablation depth of $10 \mu \mathrm{m}$. From tests 1-6, the Fig. 77 shows the center of the gear pattern's quality in comparison to each test. 


\subsection{MEMS Pattern Designs}

Following are some similar pattern designs for MEMS devices analyzed using surface mapping apparatus:
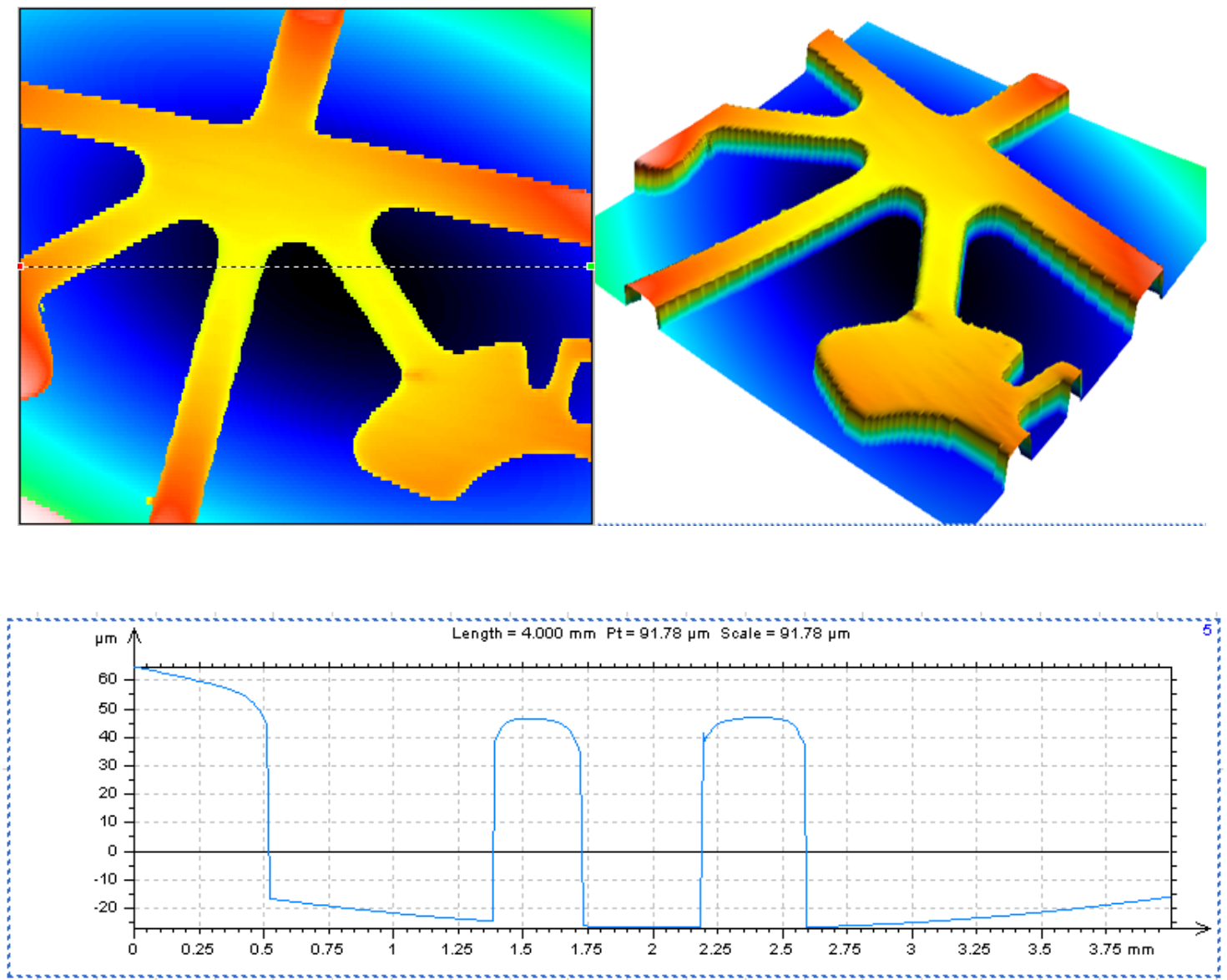

Figure $783 \mathrm{D}$ surface profile for a micro-switch contact with tolerance of $\pm 10 \mu \mathrm{m}$ material is 302S25 stainless steel (Precision 2011)

Fig. 78 shows a miniaturized medical switch contact lead frame in which the line profile shows a maximum depth of $\sim 76 \mu \mathrm{m}$. The sample is prepared using photochemical machining (PCM) process (Precision 2011). 

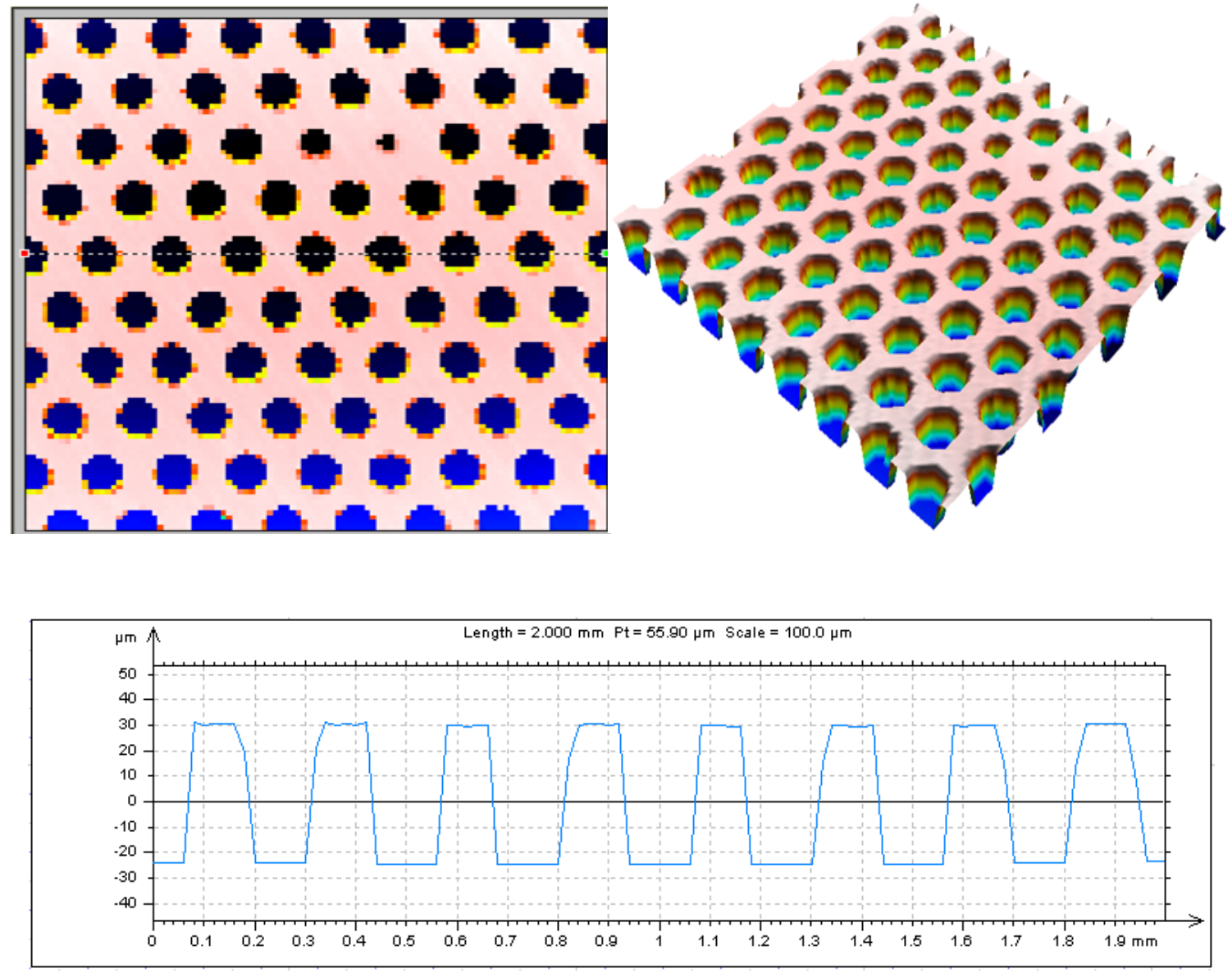

Figure 79 3D surface profile for a fine etched mesh having $30 \mathrm{holes} / \mathrm{mm}^{2}$ sample thickness of 0.05mm material is 304S15 stainless steel (Precision 2011)

Fig. 79 shows a 3D view and line profile using surface profilometer of a fine etched mesh for filtration or medical applications. From the shown line graph, a maximum depth of $\sim 60 \mu \mathrm{m}$ is obtained. 

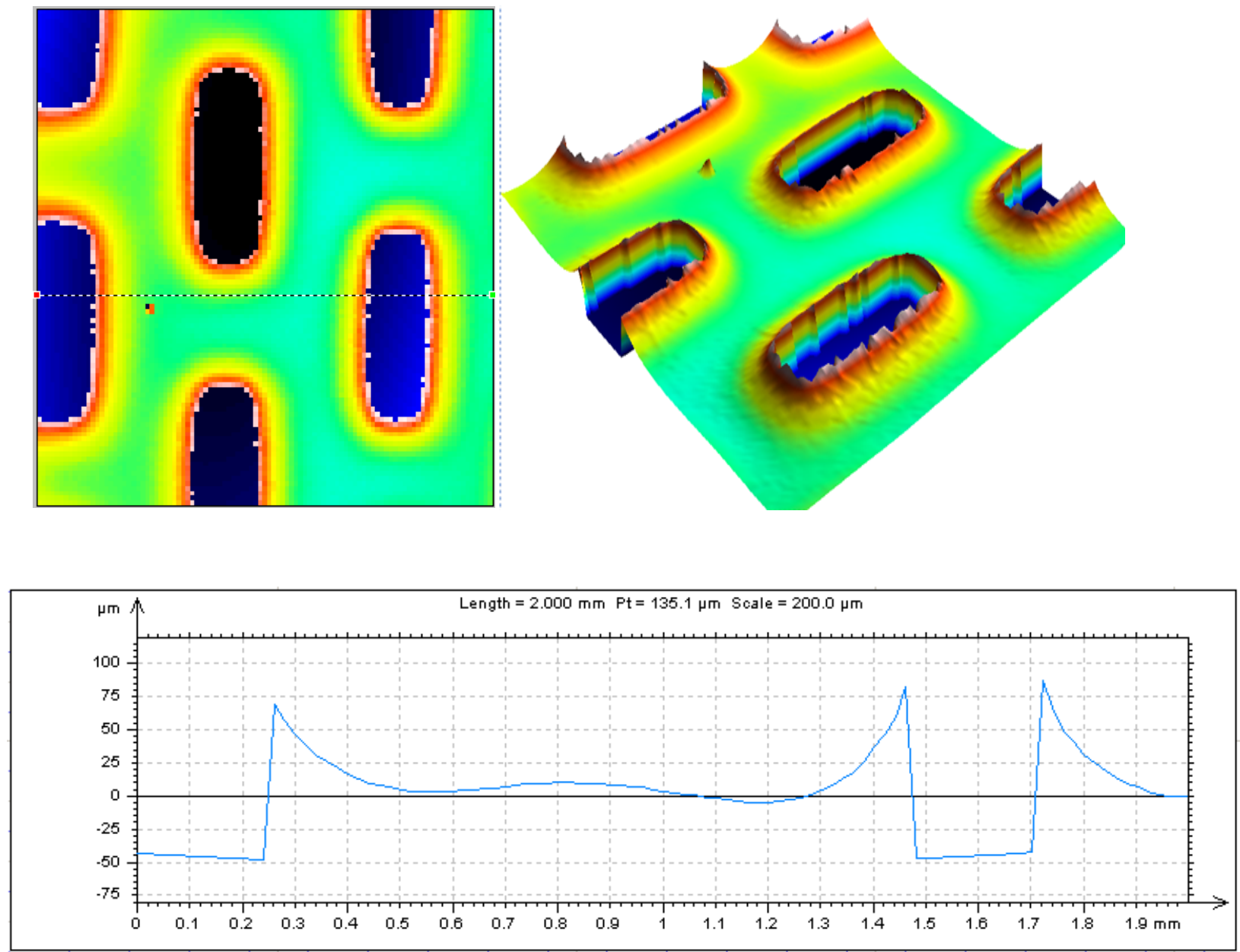

Figure 80 3D surface profile for a microfluidic channels device showing specific depth etching with sample thickness of $0.38 \mathrm{~mm}$ using $302 \mathrm{~S} 25$ stainless steel

Fig. 80 shows the 3D view and surface profile of an laser etched microfluidic device that can used for making fluid mixing areas on a MEMS device. The line profile using surface profilometer shows a maximum etch depth of $125 \mu \mathrm{m}$. 

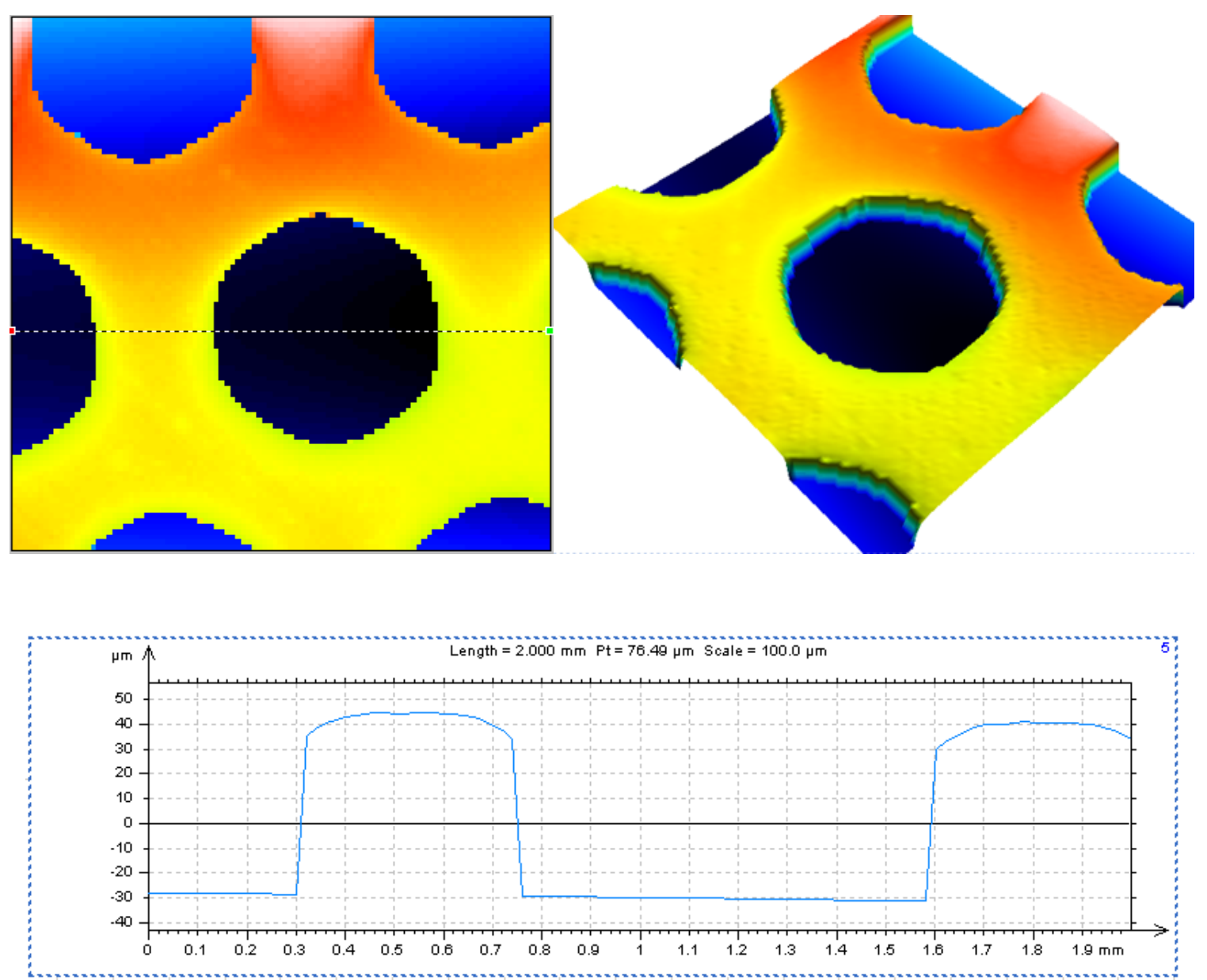

Figure 81 3D surface profile for a micro fuel cell using etched hexagonal holes with sample thickness of $0.25 \mathrm{~mm}$ and material is $316 \mathrm{~S} 11$ stainless steel

Fig. 81 shows a 3D view of a micro-fuel cell channel with hexagonal holes etched using laser for cost reduction purposes. The line profile from surface profilometer shows a maximum ablation depth of $\sim 75 \mu \mathrm{m}$. 

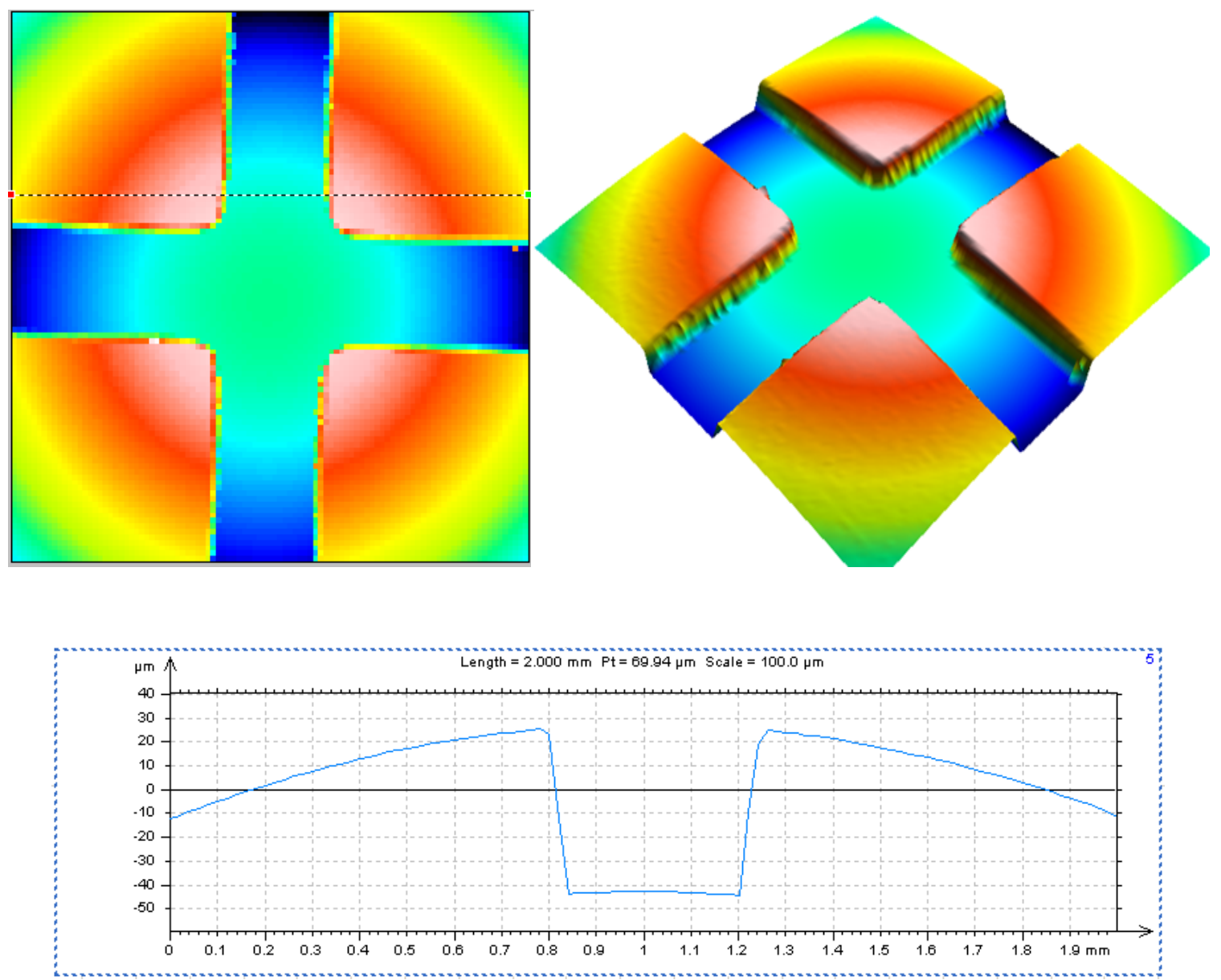

Figure 82 3D surface profile for micro-contact of a battery with sample thickness $0.20 \mathrm{~mm}$ and material is $\mathbf{1 7 - 7}$ stainless steel

Fig. 82 shows a 3D view of an aerospace battery contact structure suitable for low cost and high reliability of operation during its service life. The line profile shown in Fig. 82 from surface profilometer shows a maximum etch depth of $\sim 75 \mu \mathrm{m}$. 


\section{CHAPTER IX}

\section{SUMMARY AND CONCLUSION}

The objective of this research is to understand basic ablation mechanism involved in the excimer laser micromachining of polyurethane (PU) polymer. Polyurethane (PU) is a thermoplastic polymer which exhibits a complex ablation phenomenon under different environments; For air the instant ablation starts immediately due to low ablation threshold of polyurethane, it increases as the fluence is increased, Whereas in underwater micromachining, the ablation rate decreases with increase in fluence. The ablation mechanism of polyurethane involves various changes in the topography and surface morphology; such as chemical bi-products and heat affected zone (HAZ). From the literature and based on current experiments, it is confirmed that such kind of ablation behavior of polyurethane is due to a combination of photo-thermal and photo-chemical absorption phenomenon.

Under laser induced micromachining in air and underwater, polyurethane (PU) polymer shows the following important ablation behavior: 
- $\quad$ Low ablation threshold due to high ablation rates even at low repetition rates. Input parameters vary depending upon type of surrounding environment, e.g. air, water.

- $\quad$ For underwater environment, a large heat affected zone (HAZ) and thermally damaged surface surrounding the ablation zone is observed. For ablation to start, the surface of the polymer should be exposed to a minimum laser fluence value of $>0.25$ $\mathrm{J} / \mathrm{cm}^{2}$

- In underwater micromachining, ablation mechanism leads by formation of three zones; Heat Affected Zone (HAZ), Thermally Damaged Zone (TDZ) and Droplet Zone (DRZ). Each zone is identified by its topography and appearance. Heat affected zone (HAZ) display vibrant grey color in appearance due to localized heating of the surrounding edges of the ablated hole. Thermally damaged zone (TDZ) displays surface splitting and surface detachment due to rapid increase in temperature. Finally, the droplet zone (DRZ) formed (after TDZ) to carry out the ablation process appears to be dark in color. The change in color around HAZ surrounding the hole geometry suggests heating due to photochemical absorption of the incident laser beam.

- In air, taper angle calculations enable clear understanding of the ablation mechanism for different operating conditions. It could be considered as a good measure of quality for cross-sectional hole profile and material's micro-machinability. Higher taper angles were observed with lower fluence and vice versa. A relation of input parameters with edge geometry, minimized HAZ and maximum ablation depth can be 
developed with the help of taper angle calculations which allows fine edge geometry, minimized HAZ and very little debris formation/splashes on the periphery.

- Maximum ablation depths and lowest taper angles were observed when the specimens were micro-machined in air. No through holes were obtained in both in air and underwater due to limitations of laser equipment and thickness of polyurethane sample $(1 \mathrm{~mm})$.

- $\quad$ For 2D/3D pattern making using an excimer laser, feed rate and its effect on seam quality was determined for optimum operating parameters. Effect of mask size on seam quality showed that smaller mask sizes require higher input energies to gain the desired ablation depth. Seam quality can be greatly improved when the feed rate/step size distance was reduced in combination with increase in the repetition rates. Seam quality can be improved further using low input energy with high repetition rates.

- $\quad$ At the center of the seam, extensive melting was observed before/after ablation in the form of molten droplets of polyurethane which suggests dominance of photo-thermal mode of ablation. Due to pulse heating of the substrate, the laser beam creates a uniform high temperature gradient along its path. This temperature gradient seems to guide the molten debris from edges of the seam to the center, which may be the reason of larger diameter droplets at the center as compared to the edges of the seam. The formation of the molten droplets before and after ablation is observed due to shorter feed rate along with high fluence. 
- $\quad$ Almost any kind of 2D pattern can be etched using CimCAD ${ }^{\mathrm{TM}}$ PSO-G codes generation, while scaling of pattern to any percentage reduction in size can be made possible by using smaller proportional mask size diameters. This would help in realizing complex micromachining requirement for MEMS devices.

\subsection{Future Scope of work}

In order to further strengthen the observations, more focused approach towards ablation process and its control for polymers and non-polymers is suggested. Surface profilometer, MicroXAM and SEM technologies can be utilized to realize a clearer understanding for the ablation mechanism of polymers. For 3D pattern micromachining, the prototypes of the new micro-device designs with embedded sensors can be micromachined after determining the optimum input parameters.

In this research study, a $\mathrm{KrF}$ excimer laser (248nm) was used to investigate material removal and ablation process for polyurethane (PU) polymer. For future investigations, the research work can be extended to realize various kinds of complex pattern geometries with different kinds of polymer or composite materials. 


\section{REFERENCES}

Aldesulu, N. B., Graham and R.W. Richards, (1983) "Interstitial polymers based on a polyurethane network: "Melting points and fractional crystallinities." POLYMER, March Vol 24.

Arnold, J., Dasbach, U., Ehrfeld, W., Hesch,K., Lowe, H., (1995) "Combination of excimer laser micromachining and replication processes suited for large scale production." Applied Surface Science 86 p.p.251-258.

Angelis, C. T., Dimitriadis, C.A., Miyasaka, M., Farmakis, F.V., Kamarinos, G., Brini, J., Stoemenos, J., (1999) "Effect of excimer laser annealing on the structural and electrical properties of polycrystalline silicon thin-film transistors." Journal of Applied Physics, Vol 86, No. 8.

Alfons, V., Siau, S. J., (2006) "Optimization of microvia-technology using excimer laser for build-up layer application in microelectronics." Applie Surface Science 252 8243-8250.

Anand, S., "Laser writer, Pattern Transfer and Lift off process", Indian Institute of Science, 0424-M010.

Auvert, G., "Continuous-wave laser-assisted fabrication of microstructures in silicon microelectronics." SPIE Vol. 2045/72.

Bruan, R., Nowak,R., Hess,P., Oetzmann,H., Schmidt, C., (1989), Applied surface Science 43352.

Barun, A., Zimmer, K., Hosselbarth, B., Meinhardt, J., Bigl, F., Mehnert, R., (1998) "Excimer laser micromachining and replication of 3D optical surfaces." Applie Surface Science 127-129 911-914 
Basting, D., Pippert, K., Stamm, U., (2001) "History and Future Prospects of Excimer Laser Technology." International Symposium on Laser Precision Microfabrication.

Basting, D., Marowsky, G., (2005), "Excimer Laser Technology", Springer; 1 edition, ISBN-10: 3540200568.

Bassam, A., Cho, D., (2008) "MEMS-based multi-inlet/outlet preconcentrator coated by inkjet printing of polymer absorbents." Sensors and Actuators B133 24-32.

Bassam, M. A., Parvin, P., Sajad, S., Moghimi, A., Coster, H., (2008) "Measurement of optical and electrical properties of silicon microstructuring induced by $\mathrm{ArF}$ excimer laser at SF6 atmosphere." Applie Surface Science 254 2621-2628.

Bergmann, H. W., (1996) "Excimer laser induced surface modifications and matter interaction using double-pulse-technique (DPT)." Applied Surface Science 96-98 287-295.

Bores, L. D., (2000), "Refractive Eye Surgery", Wiley-Blackwell; 2 edition (January 15, 2000), ISBN-10:0632043644.

Christopher, E. L, (1999)"Metal Vapour Lasers: Physics, Engineering and Applications", Wiley, ISBN: 0471973874.

Christensen, C. P., (2002) "Waveguide excimer laser fabrication of 3D microstructures." SPIE Vol. 2045/141.

Colin, E. W., Julian, D.C.J., (2004), "Handbook of Laser Technology and Applications", Philadelphia ISBN-10: 0750309660

Callewaert, K., Martele,Y., Breban,L., Naessens, K., Vandaele, P., Baets, R., Geuskens, G., Schacht E., (2003) "Excimer Laser Induced Patterning of Polymeric Surfaces." Applied Surface Science (208-209).

Choi, K. H., Meijer, J., Masuzawa, T., Kim, D-T., (2004) "Excimer laser micromachining for 3D microstructure." Journal of Materials Processing Technology, 149 561566. 
Chen, Y.-T., Ma, K-J, Tseng, A.A., Chen, P.H., (2005) "Projection ablation of glassbased single and arrayed microstructures using excimer laser." Optics and Laser Technology. 37, 271-280.

Choi, Y., Choi, S-O., Shafer, R.H., Allen, M.G., (2005) "Highly Inclined Electrodeposited Metal Lines Using an Excimer Laser Patterning Technique." National Institutes of Health, NIH-BRP (EB00786-01).

Chung, C.-S,. (2007) "Chracteristics of SiCN microstructures for harch environment and high-power MEMS applications." Microelectronics Journal 38 888-893.

Coy, A. E., Viejo, F., (2010) "Effect of excimer laser surface melting on the microstructure and corrosion performance of the die cast AZ91D magnesium alloy." Corrosion Science 52 387-397.

Dyer, P. E., Farley, R.J., Giedl,R., Karnakis, D.M., (1967)."Excimer laser ablation of polymers and glasses for grating fabrication." Applied Surface Science 96-98 (1996) 537-549.

Dowling, A., Ghantasal, M., Hayes, J., Harvey, E., Doyle, D., (2001), "Excimer laser patterning of TiN film from metal sacrifical layers." Device and Process Technologies for MEMS and Microelectronics II, pp.481-488

Desbiens, J. P., Masson, P., (2007) "ArF excimer laser micromachining of Pyrex, SiC and PZT for rapid prototyping of MEMS components." Sensors and Actuators A136 554-563.

Demtroder, W., (2008). "Laser Spectroscopy", Vol.1:Basic Principles, , Springer, Fourth Edition

Dehghanpour, H. R., Parvin, P., Sajad, B., Nour-Azar, S.S.,(2009) "Dose and pressure dependence of silicon microstructure in SF6 gas due to excimer laser irradiation." Applie Surface Science 255 4664-4669.

Dale, D., (2011) "G-3 Laser Safety Manual", Oklahoma State University, Stillwater, OK.

Einstein, A., (1916) "“Zur Quantentheorie der Strahlung”." Physikalische Gesellschaft Zurich Mitteilungen Nr. 16(18) 47-62; an English translation appears in B.L. van der Waerden, ed., "Sources of Quantum Mechanics." North Holland, Amsterdam 
Evtushenko, G. S., (1999) "Metal-vapor lasers for problems of atmospheric optics." Russian Physics Journal, Vol. 42, No. 8,.

Endo, M., (2006), "Gas Lasers" ,Taylor Francis Ltd, United Kingdom ISBN:0849335531.

Gould, R. G., (1959). "The LASER, Light Amplification by Stimulated Emission of Radiation"." The Ann Arbor Conference on Optical Pumping, The University of Michigan, 15-18 June pp.128.

Gross, R. W. F., Bott, J.F., (1976), “Handbook of Chemical Lasers”, John Wiley \& Sons Inc (New York), ISBN:0471328049.

Garrison, B. J., Srinivasan, R.J., (1984), Appl. Phys. Lett. 44, 849.

Garrison, B. J., Srinivasan, R.J., (1985), Journal of Vac. Sci. Technology, A 3, 746.

Gower, M. C., Rumsby, P.T., Thomas, D.T., (1992) Proc. of SPIE, 1835133.

Gerhard, E. W., Christopher, L. T., (1993) "Polymer ablation with high power excimer laser tool." Microelectronic Engineering 20 3-14.

Grant, P. D., Tran, H., Denhoff, M. W., (1994) "Investigation of substrate temperature effect on the surface morphology of excimer laser deposited $\mathrm{YBa} 2 \mathrm{Cu} 3 \mathrm{O} 7 / \mathrm{CeO} 2$ films on sapphire." SPIE Vol. 2045/352.

Galantucci, L. M., (1998) "Excimer laser cutting: experimental characterization and 3D numerical modelling for polyester resins." Annals of the CIRP Vol. 47/1/98.

Gediminas, R., Gedvilas, M., (2005) "Processing of polymers by UV picosecond lasers." Lithuanian State Science and Studies Foundation, Project No. B-21/2005.

Garrison, B. J., Srinivasan, R.J., (2009), "Molecular dynamics model for laser ablation process." Appl. Phys. 1985 57,

Haruna, M., Tsutsumi, J., Segawa, Y., Nishihara, H., (1994) "Epiaxial growth of $\mathrm{LiNbO}_{3}$ optical-waveguide films." SPIE Vol. 2045/133.

Haglund, R. F., (1996), Journal of Applied Surface Science 96-98, 1. 
Huang, X., Li, Z., Wu, W., Chen, K., Chen, L., Liu, Z., (1996) "Microstructures and optical properties in crystallized a-Si:H multi-quantum wells using excimer laser annealing." Journal of Non-Crystalline Solids 198-200 821-824.

Judy, J. W., (2000). “Biomedical Applications of MEMS”, University of California, Los Angeles

Kuper, S., Stuke, M., (1987), Appl. Phys. B44 199.

Kahlert, H. J., Sarbach, B., Burghardt, B., Klimt B., (1992). SPIE 1835, 100

Karas, M., Sigmund, P., (1993) "Fundamental processes in sputtering o atoms and molecules (SPUT 92)." Det. Kongelige Danske Videnskabernes Selskab; Copenhagen, p.623.

Kautek, W., Kruger,J., Lenzer, M., Sartania, S., Spielmann, C., Krausz, F., (1996) "Laser ablation of dielectrics with pulse duration 20fs and 3ps." Applied Physics Letters 69 (21):3146-8.

Keoechner, W., (1999), "Solid-State Laser Engineering", Springer; 5th, rev. a. updated ed. edition ISBN-10: 3540650644.

Kruusing, A., Uusimaki, A., Petretis, B., Makarova, O., (1999) "Micromachining of magnetic materials; ." Sensors and Actuators A 74 1-3, 45-51.

Khanna, R., (2003) "MEMS fabrication perspectives from the MIT Microengine project." Surface and Coatings Technology 163-164 273-280.

Kruusing, A., (2004) "Underwater and water-assisted laser processing: Part 2 - Etching, cutting and rarely used methods." Optics and Lasers in Engineering 41 329-352.

Lowndes, D. H., Geohegan, D.B., Puretzky, A.A., Norton, D.P., Rouleau, C.M., (1996), Science 273, p.898.

Liang, W. L., Ngoi, B.K.A., Lim, L.E.N., Venkatakrishnan, K., Hee, C.W., (2003) "Micromachining of circular ring microstructure by femtosecond laser pulses." Optics \& Laser Technology 35285 - 290. 
Lee, Y.-C., Chen, C-M,. Wu, C-Y., (2005) "A new excimer laser micromachining mthod for acially symmetric 3D microstructures wtih continuous surface profiles." Sensors and Actuators A117 349-355.

Lin, J.-H., Hsu, C-C., Hsieh, W-F., Lin, K-H., (2006) "Build-up of supercontinuum in heated and unheated photonic crystal fibers using a chirped femtosecond laser." optics Communications 265 659-663.

Mackay, T. G., Weatley, D.J., Bernacca, G.M., Fisher, A.C., Hindle, C.S., (1996) "New Polyurethane Heart Valve Prosthesis: design, manufacture and evaluation." Biomaterials 17 1857-1863.

Maiman, T. H., (2000), "The Laser Odyseey"." Laser Press, California, USA.

Masubuchi, T., Fukumura, H., Masuhara, H., Suzuki, K., Hayashi, K., (2001) "LaserInduced Decomposition and Ablation Dynamics studied by Nanosecond Interferometry - A Polyurethane Film." Journal of Photochemistry and Photobiology A: Chemistry 145 215-222.

NICNAS, (2000).. "Thermoplastic Polyurethane: Full Public Report." National Industrial Chemical Notification and Assessment Scheme, FPR, PLC/146 Nov.

Onorato, M., (1985) "Gas Flow and Chemical Lasers", Plenum Pub Corp, , ISBN: 0306414783.

Pethig, R., Burt, J. P. H., Parton, A., Rizvi, N., Talary, M. S., Tame, J.A., (1998) "Development of biofactory -on-a-chip technology using excimer laser micromachining." Journal of Micromech. and Microeng., 8, 57-63.

Paterson, C., Homes,A.S., Smith, R.W., (1999) "Excimer laser ablation of microstructures: A numerical model." Journal of Applied Physics, Vol 86, No. 11 .

Papantonakis, M. R., Haglund, M.R., (2006) "Infrared-laser deposition of Teflon coatings on microstructures." Proc. of SPIE, Vol. 6111 (611104-1).

Peng, P., Sezen, A.S., Rajamani, R., Erdman, A.G., (2010) "Novel MEMS stiffness sensor for fource and elasticity measurements." Sensors and Actuators A158 1017. 
Precision, M., "Sample MEMS Designs Pack." Precision Micro Ltd. (2011), V-19902, pp.2.

Radhakrishnan, G., "Excimer laser ablation of contaminated polymide." SPIE Vol. 2045/40.

Rumbsy, P., Harvey, E., Thomas, D., Rizvi, N., "Excimer Laser Patterning of Thick and Thin Films for High Density Packaging." SPIE Vol. 3184-0277-786X/97.

Rumsby, P. T., Gower, M.C., (1991), Proc. of SPIE, 1598, 36.

Ricciardi, G., Cantello, M., Savant Aira, G., (1996) "Marking of computer keyboards by means of excimer lasers." Annals of the CIRP Vol. 45/1/1996.

Ryoichi, I., Paul Ch. van der Wilt, Barry D. van Dijk, Artyom, B., Metselaar, J.W., Beenakker, C.I.M., (2003) "Advanced excimer-laser crystallization process for single-crystalline thin film transistors." Thin Solid Films 427 77-85.

Rizvi, N. H., Rumsby, P.T., (2004), "Direct manufacture of miniature bioparticle electromanipulators by excimer laser mask projection", Oxford, U.K, University of Wales, Gwynedd. U.K.

Srinivasan, R., Braren, B., (1984), Journal of Polymer Science and Polymer Chemistry Ed. 222601.

Sutcliffe, E., Srinivasan, R., (1986), Appl. Phys. 60, 3315.

Suzuki, K., Matsuda, M., Hayashi, N., (1988) "Polymer Resist Material for Excimer Ablation Lithography." Applied Surface Science 127-129, 905-910.

Shen, B., Izquierdo, R., Meunier, M., (1994) "Laser fabrication of three-dimensional microstructures, cavities and columns" SPIE Vol. 2045/91.

Sciti, D., Melandri, C., Bellosi, A., (2000) "Excimer laser-induced microstructural changes of alumina and silicon carbide." Journal of Materials Science 35, 37993810 . 
Sedky, S., Gromova, M., Tom, V. D. J-P. C., Ann W., (2006) "Characterization of KrF excimer laser annealed PECVD SixGe1-x for MEMS post-processing." Sensors and Actuators A127 316-323.

Schaaf, P., (2010) “Laser Processing of Materials”, Springer, ISBN 978-3-642-13280-3.

Tai, Y.-C., (2001), "MEMS sensors for Biomedical Applications." NSF/ERC Industry Day, May 16-17,

Townes, C. H., (2002) "How the Laser Happened", Oxford University Press, New York, USA."

Tseng, A. A., Chen, Y-T., Ma, K-Y., (2004) "Fabrication of high-aspect-ratio microstructures using excimer laser." Optics and Lasers in Engineering 41 827847.

Tanaka, M., (2007) "An industrial and applied review of new MEMS devices features." Microelectronic Engineering 84 1341-1344.

Voronov, V. V., Dolgaev, SI, Lyalin, A.A., Shafeev, G.A., (1996) "Laser-assisted etching of the surface of polycrystalline silicon carbide by copper-vapor-laser radiation." Quantum Electron 26 (7) 621-5.

Waerden, B. L. V. D. (1967). Sources of Quantum Mechanics, Elsevier Science Publishing Co Inc.,U.S. ISBN-10: 0720401119.

Winburn, D. C., (1987), "What Every Engineer Should Know About Lasers."." Marcel Dekker Inc., New York, USA.

Weber, M. J., (1999) "'Handbook of Laser Wavelengths"." CRC Press, ISBN: 0-84933508-6.

Weng, W. C., Stephan, W.K., (1999), "Seminconductor-Laser Fundamentals: Physics of the Gain Materials", Springer; 1 edition, ISBN-10: 3540641661.

William, G. O., Shroff, Y., (2004) "Mirror-based pattern generation for maskless lithography." Microelectronic Engineering 73-74, 42-47. 
Yang, H., Pan, C-T., (2002), "Excimer laser-induced formation of metallic microstructures by electroless copper plating." Journal of Micromechanics and Microengineering, Institute of Physics Publishing, 12 157-161.

Yoshihisa, U., Tetsuya W., Yoshinori, Y. Kazuaki, T., Hiroshi, K., and Kimio I., (2009) "405nm Laser Thermal Lithography of $40 \mathrm{~nm}$ Pattern Using Super Resolution Organic Resist Material." Applied Physics Express 2126502.

Zimmer, K., Hirsch, D., Bigl, F., (1996) "Excimer laser machining for the fabrication of analogous microstructures." Applie Surface Science 96-98 425-429.

Zhigilei, L. V., Kodali, P. B.S., Garrison, P. B.S.,(1998) "A microscopic view of laser ablation." Journal of Phys. Chem. B 1998, 102. 2845-2853.

Zheng, H., Gan, E., Lim, G.M., (2001) "Investigation of laser via formation technology for the manufacturing of high density substrates." Optics and Lasers in Engineering 36 355-371. 


\section{VITA}

Sarabpreet Singh

Candidate for the Degree of

Master of Science

\section{Thesis: MICROMACHINING OF POLYURETHANE (PU) POLYMER USING A} KrF EXCIMER LASER (248NM)

Major Field: Mechanical Engineering

Biographical:

Personal Data: Born in Hoshiarpur, Punjab, India, on April $24^{\text {th }}$, 1985, the son of S. Jasvir Singh and Tarlochan Kaur

Education: Received Bachelor of Science degree in Mechanical Engineering from Sant Longowal Institute of Engineering and Technology, India in June 2007. Completed the requirements for Master of Science degree with a major in Mechanical and Aerospace Engineering at Oklahoma State University in May 2011.

Experience: Graduate Teaching Assistant in the School of Industrial Engineering Management, and Mechanical and Aerospace Engineering, Oklahoma State University, Stillwater, Oklahoma, August 2008 - Present.. 
Title of Study: MICROMACHINING OF POLYURETHANE (PU) POLYMER USING A KrF EXCIMER LASER (248NM)

Major Field: Mechanical Engineering

\section{Scope and Method of Study:}

Excimer laser micromachining has generated research interest and commercial applications in the last decade for numerous application areas. Polyurethane (PU) polymer, due to its biocompatibility, weather resistance and favorable physical properties such as good flex-life, temperature resistance, electrical insulation and high tear resistance finds a number of applications in medical implants, and as a prototype material for structural components in MEMS devices. Conventional machining operations are unsuitable for precision cutting, marking or shaping the polyurethane material due to its polymeric nature and sensitivity for high temperature exposure. Excimer lasers work in UV wavelengths and provide a method to precisely micromachine polymers and other soft materials with a wide range of parameters to control, namely, number of pulses, fluence, repetition rates. A KrF Excimer laser (wavelength $=248 \mathrm{~nm}, \mathrm{FWHM}=25 \mathrm{~ns}$ ) is employed in this research work for micromachining of polyurethane and pattern design for potential MEMS applications.

\section{Findings and Conclusions:}

The micromachined surfaces of polyurethane polymer are examined using optical microscopy, surface profiler and MicroXAM laser interference microscope. The effect of various operating parameters, including fluence per pulse, number of pulses, repetition rates and environment on the resulting geometries and ablation behavior are investigated. Micromachining was conducted in air and under water environments with different mask sizes and pattern geometries. It was observed that, for air environment, the ablation rate was $0.18 \mu \mathrm{m} / \mathrm{pulse}$, and for underwater environment, the ablation rate was $0.07 \mu \mathrm{m} / \mathrm{pulse}$. A combination of photo-thermal and photo-chemical ablation mechanism was attributed in the material removal for polyurethane polymer. Micro-gears (up to $360 \mu \mathrm{m}$ diameter) were etched in polyurethane with several similar geometries used in MEMS devices such as micro-fluidic channels and microcircuits. 
